\title{
MEASUREMENT OF THE TOP QUARK MASS IN DILEPTON FINAL STATES WITH THE NEUTRINO WEIGHTING METHOD AT THE DØ EXPERIMENT
}

Approved by:

Dr. Robert Kehoe

Dr. Roberto Vega

Dr. Stephen Sekula

Dr. Michael Lattman 


\section{MEASUREMENT OF THE TOP QUARK MASS IN DILEPTON FINAL STATES WITH THE NEUTRINO WEIGHTING METHOD AT THE D EXPERIMENT}

A Dissertation Presented to the Graduate Faculty of the

Dedman College

Southern Methodist University

in

Partial Fulfillment of the Requirements

for the degree of

Doctor of Philosophy

with a

Major in Experimental Particle Physics

by

Yuriy Ilchenko

(B.S., Odessa National University, 2003)

(M.S., Odessa National University, 2004)

Dec 15, 2012 


\section{ACKNOWLEDGMENTS}

Without a doubt, graduate study is an interesting and rewarding experience. It has played a major role in my life and changed me in a good direction in many respects. This process, however, would not be possible without the help and support of several people.

First, I would like to thank my advisor, Professor Robert Kehoe. Not only was he a great physicist and thoughtful advisor who guided me through the research, but also an outstanding mentor. Numerous lessons I learned from him became particularly valuable assets in my life. I truly appreciate his enormous patience, persistence, understanding and encouragement while working with me. His passion for success was always inspiring and motivating to me.

Many thanks are owed to Peter Renkel for his scientific advice and many insightful discussions about the top quark, physics and science in general. I especially thank Haleh Hadavand who I worked with on the data quality monitoring project at the ATLAS experiment. She helped me to strengthen my computing skills and establish a solid basis for subsequent self-development.

I am thankful the theory professors: Kent Hornbostel, Simon Dalley, and Roberto Vega. I have enjoyed their lecture a lot while taking classes at SMU. In particular, Kent's lectures on Quantum Field Theory provided an elegant and remarkably clear introduction to the theory of particle physics. For reading the thesis and giving their insightful comments, I also would like to thank the dissertation committee members: Stephen Sekula, Roberto Vega and Michael Lattman.

Special thanks go to SMU physics department staff, especially Shirley Melton and 
Carol Carroll. They have helped me with various issues so many times. Also Carroll's coffee was a big help for starting a new day.

I would like to thank Ryszard Stroynowski, the SMU ATLAS professor. His support at the earlier stage of my research along with words of wisdom and tremendous insights encouraged me to pursue excellence in high energy physics. I would like to thank my colleagues from the $\mathrm{D} \varnothing$ collaboration. In particular, members of the double-top group for valuable suggestions and comments on the analysis. I am also indebted to Christian Schwanenberger and Dmitri Denisov for their support, guidance and encouragement.

I am very thankful to my friend Azeddine Kasmi for his support during the difficult times, and for his insight and precious advice regarding anything I asked him about life and science. Whenever lost or disappointed, I always was able to turn to him for help. I am also thankful for many lively discussions and remember many great moments we shared together when I was at Fermilab. I have enjoyed his friendship so much.

I owe a great debt of gratitude to Vladimir Ajaev, Oleg Volkov, Tatyana Savchuk, Maksim Mayarovich, Robert Ishmukhametov and Renat Ishmukhametov. They have been wonderful friends who made my graduate school years unforgettable. They always were willing to help and support me when I was in need. I also want to acknowledge my good friends from Chicago and Dallas for memorable graduate school experience: Evgeny Toropov, Anna and Andrey Elagin, Sergey Koshelev, Anna Kuznetsova, Ekaterina and Alexander Mukhammadiyev, Igor Kanash, Yegor Aushev, Alexander Verkheev, Olga Gogota, Zhihua Liang, Huanzhao Liu. I would like to thank my friend from Odessa Andrey Mulyava. Even though he was so far away, his help and friendship cannot be underestimated. I am also grateful to Elena Odnokoz for her belief in me and the support at the final stage of my graduate study.

I thank my father Nikolay Ilchenko for his word of encouragement and material support. He taught me math when I was in high school, advised me to think critically 
and eventually sparked my love to science.

And lastly but most importantly, I wish to express my deep gratitude to Aleksandra Mamedova. Your infinite love, tremendous patience and unconditional sacrifices, invaluable support before and during the first years of the graduate school were the sources of inspiration, encouragement and energy, guidance through hurdles of the study and life as a whole. Without having you as a best friend, my $\mathrm{PhD}$ endeavor would not even begin. 
Measurement of the Top Quark Mass in Dilepton Final States

with the Neutrino Weighting Method at the DØ experiment

Advisor: Professor Robert Kehoe

Doctor of Philosophy degree conferred Dec 15, 2012

Dissertation completed Dec 07, 2012

The top quark is the heaviest fundamental particle observed to date. The mass of the top quark is a free parameter in the Standard Model (SM). A precise measurement of its mass is particularly important as it sets an indirect constraint on the mass of the Higgs boson. It is also a useful constraint on contributions from physics beyond the SM and may play a fundamental role in the electroweak symmetry breaking mechanism. I present a measurement of the top quark mass in the dilepton channel using the Neutrino Weighting Method. The data sample corresponds to an integrated luminosity of $4.3 \mathrm{fb}^{-1}$ of $p \bar{p}$ collisions at Tevatron with $\sqrt{s}=1.96 \mathrm{TeV}$, collected with the D $\varnothing$ detector. Kinematically under-constrained dilepton events are analyzed by integrating over neutrino rapidity. Weight distributions of $t \bar{t}$ signal and background are produced as a function of the top quark mass for different top quark mass hypotheses. The measurement is performed by constructing templates from the moments of the weight distributions and input top quark mass, followed by a subsequent likelihood fit to data. The dominant systematic uncertainties from jet energy calibration is reduced by using a correction from $\ell+$ jets channel. To replicate the quark flavor dependence of the jet response in data, jets in the simulated events are additionally corrected. The result is combined with our preceding measurement on $1 \mathrm{fb}^{-1}$ and yields $m_{t}=174.0 \pm 2.4$ (stat.) \pm 1.4 (syst.) GeV. 


\section{TABLE OF CONTENTS}

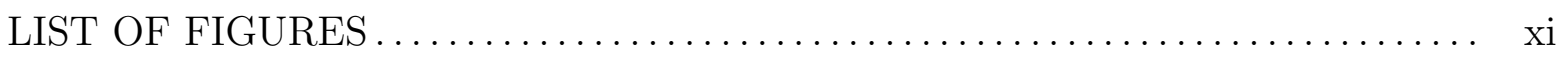

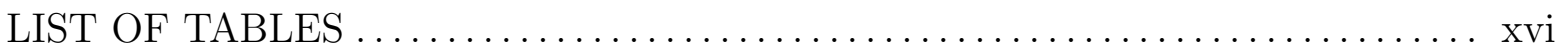
CHAPTER

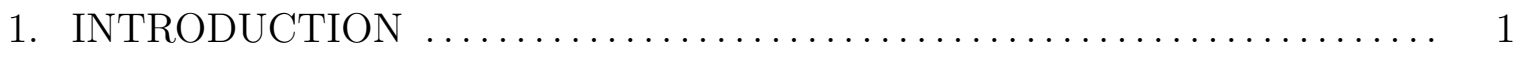

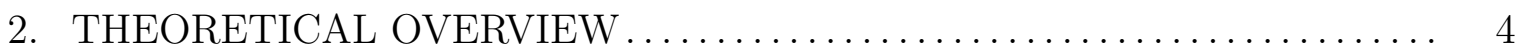

2.1. The Standard Model $\ldots . \ldots \ldots \ldots \ldots \ldots \ldots \ldots \ldots \ldots \ldots \ldots \ldots \ldots, 4$

2.1.1. The Electroweak Theory $\ldots \ldots \ldots \ldots \ldots \ldots \ldots \ldots \ldots \ldots \ldots$

2.1.2. Spontaneous Symmetry Breaking and The Higgs Mechanism . . 12

2.1.3. Quantum Chromodynamics ........................ 16

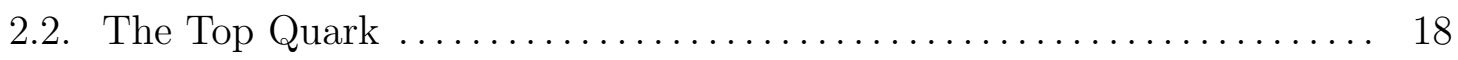

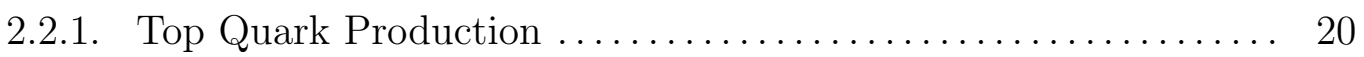

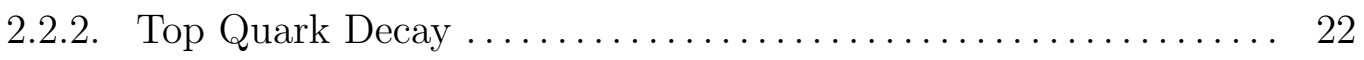

2.2.3. The Definition of Mass ........................... 25

2.2.4. Top Quark in SM and beyond SM $\ldots \ldots \ldots \ldots \ldots \ldots \ldots \ldots .26$

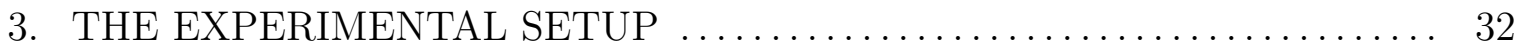

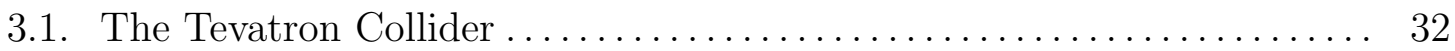

3.1.1. Proton Creation and Acceleration ...................... 34

3.1.2. Antiproton Creation and Acceleration ................. 37

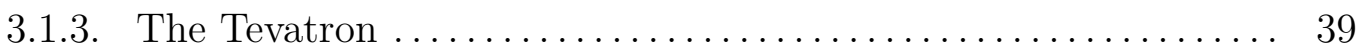

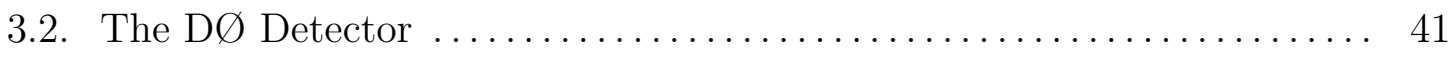

3.2.1. $\quad \varnothing$ Coordinate System........................... 41 
3.2.2. Central Tracking System $\ldots \ldots \ldots \ldots \ldots \ldots \ldots \ldots \ldots \ldots \ldots \ldots \ldots \ldots$

3.2.2.1. Silicon Microstrip Tracker ..................... 43

3.2.2.2. Central Fiber Tracker $\ldots \ldots \ldots \ldots \ldots \ldots \ldots \ldots \ldots \ldots$

3.2.2.3. Solenoidal Magnet $\ldots \ldots \ldots \ldots \ldots \ldots \ldots \ldots \ldots \ldots . \ldots \ldots$

3.2.3. Calorimetry .................................. 47

3.2.3.1. Uranium/Liquid-Argon Calorimeter ............ 47

Electromagnetic Shower $\ldots \ldots \ldots \ldots \ldots \ldots \ldots \ldots \ldots$

Hadronic Shower $\ldots \ldots \ldots \ldots \ldots \ldots \ldots \ldots \ldots \ldots \ldots \ldots$

3.2.3.2. Preshower Detectors $\ldots \ldots \ldots \ldots \ldots \ldots \ldots \ldots \ldots \ldots \ldots$

3.2.3.3. Inter Cryostat Detector $\ldots \ldots \ldots \ldots \ldots \ldots \ldots \ldots \ldots \ldots$

3.2.4. Muon System ................................ 56

3.2.4.1. Drift Tubes ............................. 57

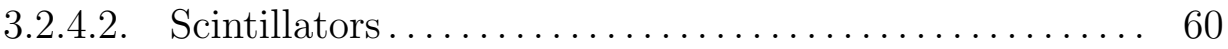

3.2.4.3. Toroidal Magnet $\ldots \ldots \ldots \ldots \ldots \ldots \ldots \ldots \ldots \ldots \ldots . \ldots 2$

3.2.5. Triggering and Data Acquisition System ................ 63

3.2.5.1. Level 1 Triggers............................ 64

3.2.5.2. Level 2 Triggers.......................... 65

3.2.5.3. Level 3 Triggers........................... 65

3.2.5.4. Data Acquisition System ...................... 66

4. DATA SAMPLE AND SIMULATION $\ldots \ldots \ldots \ldots \ldots \ldots \ldots \ldots \ldots \ldots \ldots \ldots$

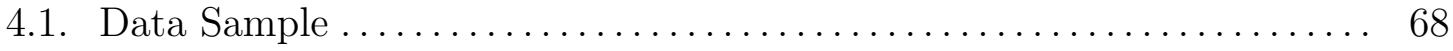

4.1.1. Data Time Periods ................................ 69

4.1.2. Data Quality ................................... 69

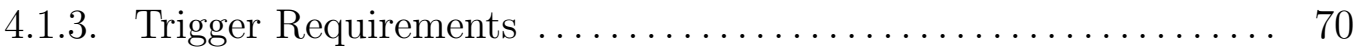


4.2. Monte Carlo Simulation $\ldots \ldots \ldots \ldots \ldots \ldots \ldots \ldots \ldots \ldots \ldots \ldots \ldots \ldots \ldots \ldots \ldots \ldots \ldots \ldots \ldots$

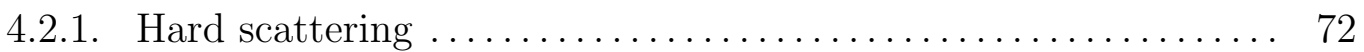

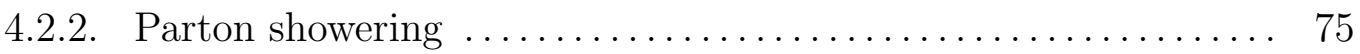

4.2.3. Hadronization ............................... 76

4.3. Monte Carlo Samples.............................. 78

4.3.1. Signal Sample................................ 78

4.3.2. Background Samples ......................... 79

5. DATA RECONSTRUCTION AND OBJECT IDENTIFICATION ....... 80

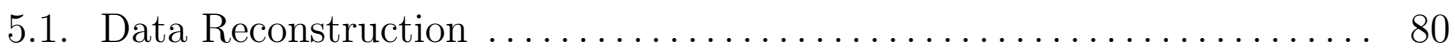

5.2. Object Identification and Reconstruction.................. 82

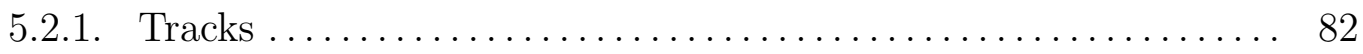

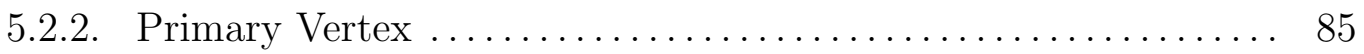

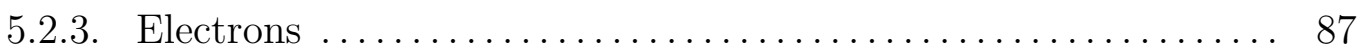

5.2.3.1. Cluster Reconstruction and Requirements ........ 88

5.2.3.2. Electron Quality Variables ................ 89

5.2.3.3. Electron Energy Scale................... 92

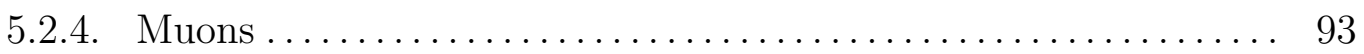

5.2.4.1. Muon Quality Variables .................... 94

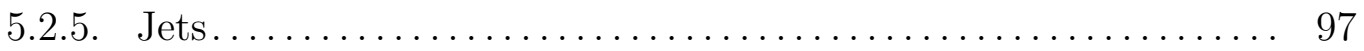

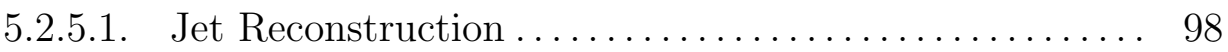

5.2.5.2. Jet Quality Variables..................... 101

5.2.6. Missing Transverse Energy . . . . . . . . . . . . . . . . . 103

5.2.6.1. Missing Transverse Energy Significance ............. 104 


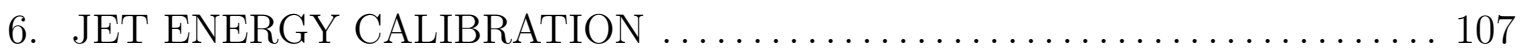

6.1. Standard Jet Energy Scale Correction $\ldots \ldots \ldots \ldots \ldots \ldots \ldots \ldots \ldots \ldots \ldots$

6.1.1. Missing $E_{T}$ Projection Fraction Method $\ldots \ldots \ldots \ldots \ldots \ldots \ldots . \ldots 111$

6.1.2. Sample Purity Estimation .......................... 115

6.1.2.1. Hollow Cone Template Method ................... 116

6.2. Analysis-Specific Jet Energy Scale Corrections ................... 118

6.2.1. Flavor-Dependent Jet Response Correction ............... 120

6.2.2. $\ell+$ jets Jet Energy Scale Correction ..................... 123

6.2.2.1. Adopting the $\ell+$ jets Jet Energy Scale ............ 125

7. THE NEUTRINO WEIGHTING METHOD ....................... 128

7.1. Kinematic Reconstruction .............................. 128

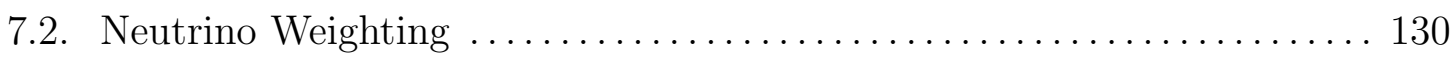

7.2.1. Weight Calculation............................... 132

7.2.2. Neutrino Pseudorapidity Choices ...................... 136

7.2.3. Neutrino Pseudorapidity Modeling....................... 139

7.3. Maximum Likelihood Method ................................ 139

7.3.1. Probability Density Templates.......................... 142

7.4. Testing and Calibration using Pseudoexperiments ................. 143

7.4.1. Mass Measurement Calibration ......................... 144

7.4.1.1. High $\chi^{2}$ of Calibration Curve.................. 145

7.4.2. Statistical Uncertainty Calibration.................... 147

7.4.2.1. Mass Calibrated Correction ................... 147

7.4.2.2. Pull Width Calibration ...................... 148 
8.1. Event Selections ....................................... 159

8.2. Data Measurement ...................................... 163

8.3. Systematic Uncertainties .................................. 164

8.3.1. Treatment of systematic uncertainties $\ldots \ldots \ldots \ldots \ldots \ldots \ldots \ldots$

8.3.2. Jet Energy Scale ................................ 170

8.3.2.1. Overall scale ............................ 170

8.3.2.2. Residual scale............................. 172

8.3.2.3. Flavor Dependence........................... 173

8.3.3. QCD Modeling..................................... 174

8.3.3.1. ISR/FSR ............................ 174

8.3.3.2. Color reconnection ......................... 175

8.3.3.3. Higher order effects ......................... 175

8.3.3.4. $b$-quark fragmentation...................... 176

8.3.3.5. PDF uncertainty ......................... 176

8.3.4. Object Reconstruction and Identifications............... 177

8.3.4.1. Electron energy scale........................ 177

8.3.4.2. Muon energy scale .......................... 177

8.3.4.3. Muon transverse momentum resolution ............. 178

8.3.4.4. Jet energy resolution ......................... 178

8.3.4.5. Jet identification ........................... 179

8.3.5. Systematics of the Method ............................ 179

8.3.5.1. Calibration ................................. 179

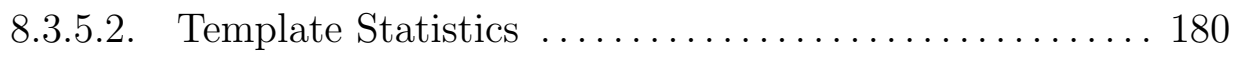




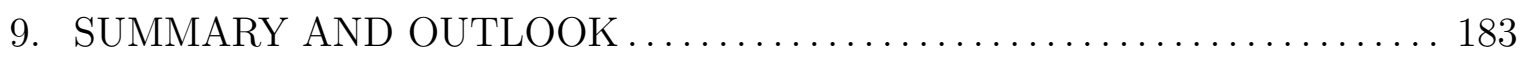

\section{APPENDIX}

A. MUON QUALITY REQUIREMENTS $\ldots \ldots \ldots \ldots \ldots \ldots \ldots \ldots \ldots \ldots \ldots \ldots \ldots$

B. MUON ISOLATION WORKING POINTS ..................... 190

C. THE PULL VARIABLE PROBABILITY DENSITY FUNCTION . . . . . . 191

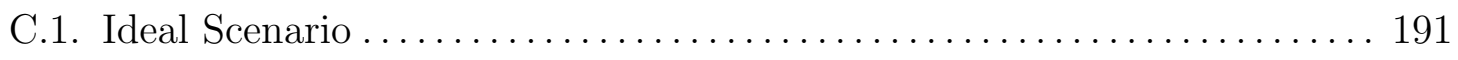

C.2. Non-Ideal Scenario .................................... 193

D. PULL-CORRECTED CALIBRATED STATISTICAL UNCERTAINTY . . . 194

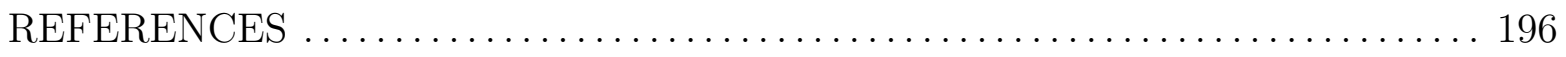




\section{LIST OF FIGURES}

Figure

2.1. Fundamental particles of the Standard Model. Matter particles are the quarks and leptons. Force mediators are the gauge bosons. . . . . . 7

2.2. Second-order Feynman diagram representing $e^{+} e^{-}$pair annihilation to a virtual photon $\gamma$ with subsequent re-emission. ............... 10

2.3. The leading order Feynman diagrams for $t \bar{t}$ production: quark-antiquark annihilation $(q \bar{q})$ and gluon-gluon fusion $(g g) . \ldots \ldots \ldots \ldots \ldots \ldots \ldots . \ldots \ldots$

2.4. The leading-order Feynman diagrams for the electroweak production of a single top quark: $s$-channel (left), $t$-channel (right). $\ldots \ldots \ldots \ldots \ldots 22$

2.5. The $t \bar{t}$ decay modes and their respective branching ratios. $\ldots \ldots \ldots \ldots \ldots 24$

2.6. Representative Feynman diagrams for the radiation corrections. . . . . . . 27

2.7. The indirect constraints on $m_{t}$ and $m_{W}$ (dashed red contour) from LEPI and SLD data, and the direct measurements based on LEP-II and Tevatron data (solid blue ellipse) as of summer 2009. Green bands are the SM relationship for the masses as a function of the Higgs mass not excluded by the direct searches $\left(114<m_{H}<158 \mathrm{GeV}\right.$

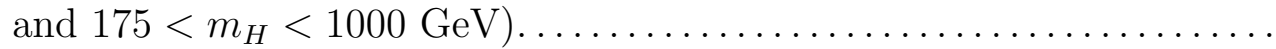

2.8. Experimental and theoretical $t \bar{t}$ cross section $\sigma_{t \bar{t}}$ as a function of the top quark mass $m_{t}$. The colored dashed lines represent the uncertainties for the theoretical predictions from the choice of the PDF, the renormalization and factorization scales. The point is the measured combined $\sigma_{t \bar{t}}$ evaluated for $m_{t}=172.5 \mathrm{GeV}$. The black curve shows experimental $\sigma_{t \bar{t}}$ as a function of $m_{t}$. The gray band corresponds

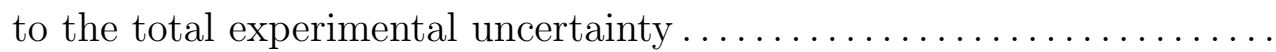

3.1. Tevatron Accelerator Complex. Many steps are required to bring into collision proton and antiproton beams. The locations of the $\mathrm{D} \varnothing$ and $\mathrm{CDF}$ detectors are indicated. 
3.2. Schematic view of the magnetron, the $H^{-}$ion source used at Fermilab. ... 34

3.3. Electric circuit of the two cascade Cockcroft-Walton generator.......... 35

3.4. The picture of the Cockcroft-Walton pre-accelerator at Fermilab. ........ 36

3.5. Superconducting RF resonator designed at Fermilab............. 37

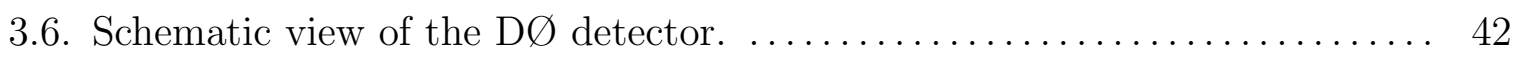

3.7. Schematic view of the $\mathrm{D} \varnothing$ central tracking system............. 44

3.8. Schematic view of the $\mathrm{D} \varnothing$ silicon microstrip tracker. ............ 45

3.9. Schematic view of the $\varnothing \varnothing$ Central Fiber Tracker. ................ 47

3.10. A view of magnetic field in the detector (in $\mathrm{kG}$ ). Both the toroidal and solenoidal magnets are on. ......................... 48

3.11. Schematic view of the $\mathrm{D} \varnothing$ Liquid Argon Calorimeter. ............ 50

3.12. Schematic view of the liquid argon cell for the calorimeter. $\ldots \ldots \ldots \ldots .51$

3.13. Schematic view of the portion of the U/LAr showing layers and tower

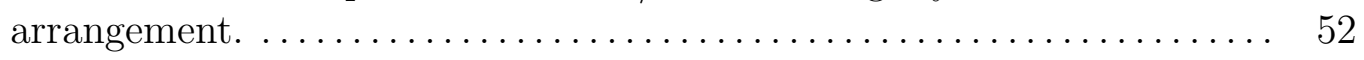

3.14. Schematic view of an electromagnetic shower. $\ldots \ldots \ldots \ldots \ldots \ldots \ldots \ldots .53$

3.15. Schematic development of hadronic shower in calorimeter. ......... 53

3.16. Schematic view of cross section of the CPS and FPS scintillator strips. ... 54

3.17. Cross section view of the ICD tiles on the endcap cryostats. The rectangles represent an assembly where ICD electronics is installed. . . . . 56

3.18. Schematic view of the muon wire chambers. $\ldots \ldots \ldots \ldots \ldots \ldots \ldots \ldots$

3.19. Schematic view of a mini drift tube. $\ldots \ldots \ldots \ldots \ldots \ldots \ldots \ldots \ldots \ldots \ldots$

3.20. Schematic view of the muon scintillation detectors.............. 60

3.21. The picture of the trigger scintillation counters in the C-layer........ 62

3.22. Overview of the $\mathrm{D} \varnothing$ trigger and data acquisition systems. $\ldots \ldots \ldots \ldots \ldots$ 
4.1. Schematic view of hard scattering process with subsequent parton show-

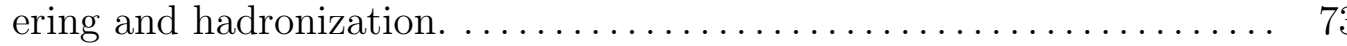

4.2. Left: The string fragmentation model diagram. The pink contour represents a string between two quarks kinked by the gluons and the green contour is a single straight string between two quarks. Right: The cluster fragmentation model diagram. The brown contours represent clusters formed by pairs of two quarks.

5.1. Track construction diagram using the Alternative Algorithm technique. The track candidate (green line) is based on the three consecutive

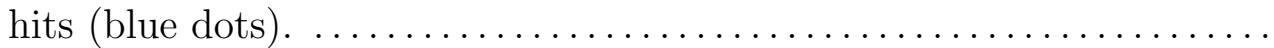

5.2. The illustration of the Hough transformation for a single muon of 1.5 GeV leaving 5 hits in the SMT. (a) Circular trajectories passing through the given hit. (b) A line in the $(\rho, \phi)$ space that corresponds to the set of trajectories for the hit. (c) Five hits correspond to the five lines crossing at one point in $(\rho, \phi)$ space. (d) $2 \mathrm{D}$ histogram with the peak giving track candidate parameters.

6.1. Sketch of a parton evolution into a jet in the calorimeter. The original parton is the result of hard scattering $p \bar{p}$ interaction. ............ 109

6.2. The main Feynmann diagrams of the direct photon production in $\gamma+$ jet events.

6.3. The jet response calculated for $J C C B$ jets in RunIIa MC simulation. The JCCB jet is a jet with the cone size $\Delta R=0.5$. The jets with the cone size $\Delta R=0.7$ is called JCCA. ................. 114

6.4. The hollow cone track variable distributions in the region with $0.0<$ $\eta_{\text {jet }}<0.4$ and $40<E^{\prime}<60 \mathrm{GeV}$. The distribution for the data is shown by the filled circles, while MC simulations are: signal template (squares), background template (triangles), and $\mathrm{MC}$ fit to the data (empty circles).

6.5. Sample purity calculated with the hollow cone method in the different $\eta_{\text {jet }}$ regions. The purity is shown in the black circles with corresponding error bars reflecting the uncertainty. The parametrization fit is the solid red line. Dashed red bands indicate the statistical

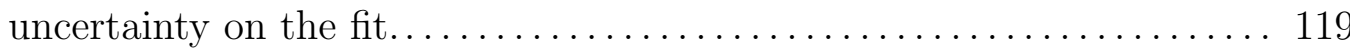

6.6. Number of particles in a jet initiated from gluon (blue) and quark (red)... 121 
6.7. Fitted Gaussian contours of equal probability for the two-dimensional likelihoods as a function of $m_{t}$ and $k_{J E S}$. The contours correspond to a $1-\sigma, 2-\sigma$, and $3-\sigma$ statistical uncertainty on $m_{t} \ldots \ldots \ldots \ldots \ldots 12$

6.8. The double ratio, $R_{2 \ell}^{b}$, is plotted vs $b$-jet transverse momentum. The value of the double ratio in each bin is shown in black circles with corresponding error bars reflecting the uncertainty. Dashed blue line is the asymptotic value of the double ratio. ................ 126

7.1. Schematic representation of $e \mu$ dilepton decay mode in $t \bar{t}$ event produced in hard-scattering interaction of quark-antiquark pair. ....... 129

7.2. Example of neutrino rapidity distribution with the top quark mass $m_{t}=$ $172 \mathrm{GeV}$ as simulated by ALPGEN. The smooth black curve is a Gaussian fit. ......................................... 136

7.3. Example of the weight distribution for simulated $t \bar{t}$ event with $m_{t}=172$ $\mathrm{GeV}$.

7.4. The weight distribution averaged over all events in MC $t \bar{t}$ sample of masses $160 \mathrm{GeV}$ (red), $170 \mathrm{GeV}$ (green) and $180 \mathrm{GeV}$ (blue). ....... 137

7.5. Example of the weight distributions obtained with different numbers of rapidity bins: (a) $N=10$, (b) $N=30$, (c) $N=70$, (d) $N=200 \ldots \ldots 152$

7.6. Width of the Gaussian fit to the neutrino rapidity distribution as a function of the top quark mass. The black line is a linear fit......... 153

7.7. Slice of the probability density template $h_{s}\left(\mu_{w}, \sigma_{w} \mid m_{t}\right)$ for $m_{t}=170$ $\mathrm{GeV}$ for $e \mu$ channel. ................................... 154

7.8. The combined background probability density template $h_{b}\left(\mu_{w}, \sigma_{w}\right)$ for $e \mu$ channel. ........................................ 15

7.9. $-\ln (L)$ for a $\mathrm{MC}$ simulated pseudoexperiment for $e \mu$ channel. . ......... 155

7.10. An example of the calibration points and the linear fit applied for ee $(t o p)$ channel. The effects contributing to a high $\chi^{2}$ are not accounted for.

7.11. The calibration points and the linear fit applied for ee (top), e $\mu$ (center) and $\mu \mu$ (bottom) channel. An additional uncertainty due to finite template statistics is added in quadrature with statistical uncertainty at every mass point. 
7.12. Pull distribution for the input top quark mass $m_{t}=170 \mathrm{GeV}$ in the $e \mu$ channel. The superimposed curve is the fit using a Gauss function..... 157

7.13. The pull calibration points and the linear fit applied for ee (top), e $\mu$ (center) and $\mu \mu$ (bottom) channel......................... 158

8.1. The $-\ln \left(L\left(m_{t}\right)\right)$ as a function of input top quark mass for the ee (top left), $e \mu$ (top right), $\mu \mu$ (bottom left) channels and their combination (bottom right). The black curve is a parabolic fit near the minimum

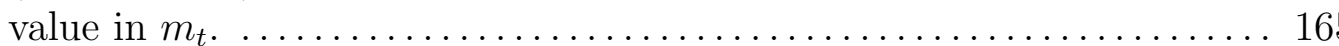

8.2. Expected calibrated and pull-corrected statistical uncertainty distributions for the ee (top left), e $\mu$ (top right), $\mu \mu$ (bottom left) channels and their combination (bottom right. The distributions are obtained from pseudoexperiment testing for the input top quark mass $m_{t}=170 \mathrm{GeV}$. Arrows indicate measured top quark mass in

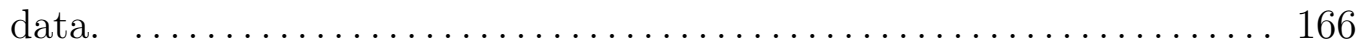

9.1. Summary of the top quark mass measurement in different decay channels at $\mathrm{D} \varnothing$. The $\mathrm{D} \varnothing$ combination and Tevatron average of the top quark mass [131]. The mass extracted from the cross section measurement is shown for comparison and has not been used in the combination.

9.2. The indirect constraints on $m_{t}$ and $m_{W}$ (orange ellipse) from LEP-II and Tevatron data as of July 2012. Green bands are the SM relationship for the masses as a function of the Higgs mass not excluded by the direct searches $\left(115.5<m_{H}<127 \mathrm{GeV}\right.$ and $\left.600<m_{H}<1000 \mathrm{GeV}\right)$. 186 


\section{LIST OF TABLES}

Table

Page

2.1. Charge and lepton flavor for three generations of leptons. . . . . . . . . . 6

2.2. Charge and flavor quantum numbers for three generations of quarks. $\ldots \quad 6$

2.3. The fundamental interactions in nature. The strength of an interaction is given in relationship to the strong interaction which is taken as

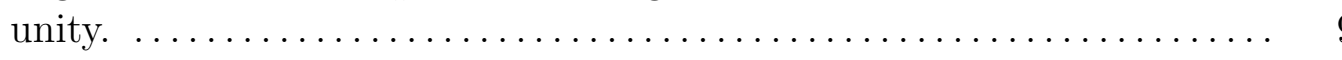

6.1. Average number of particles in a jet for $\ell+$ jets and dilepton channels. . . 127

7.1. The slope $(\alpha)$ and offset $(\beta)$ calibration parameters with the statistical uncertainty for the dilepton channels and the combination.......... 148

7.2. The pull-calibrating correction $(\lambda)$ with the statistical uncertainty for the dilepton channels and the combination................. 151

8.1. The global variables event selections for $t \bar{t}$ dilepton channels. An entire event is rejected if at least one of the variables has a value below

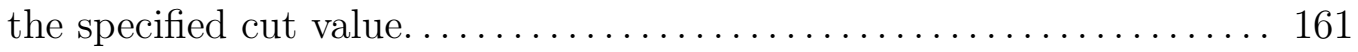

8.2. The signal and background kinematic reconstruction efficiencies for he $e \mu$ channel with no $H_{T}$ cut applied. The efficiency for signal has been estimated on a $t \bar{t}$ sample of input top quark mass, $m_{t}=170$ $\mathrm{GeV}$.

8.3. Expected and observed event yields and their statistical uncertainty for $t \bar{t}$ dilepton channels after kinematic reconstruction and global selections applied. The expected yields have been derived on simulated MC signal and background sample. The instrumental background and its yield are derived from the data. The observed event yield is obtained using the events from data. ................... 163

8.4. Summary of the systematic uncertainties of all dilepton channels and their combination dived in categories. The uncertainties are quoted in $\mathrm{GeV}$. 
8.5. Calibration systematic uncertainty with template statics accounted for

(right), and without it (left). 181 
This dissertation is dedicated to humankind and its marvelous achievements in sciences and humanities. 


\section{Chapter 1}

\section{INTRODUCTION}

The questions - what matter is made of, and what fundamental forces of the nature are - have been challenging humanity over many centuries. First concepts of matter come from the age of ancient Greece about two thousand year ago. In the atomist philosophy of Democritus, it was postulated that matter is made of indivisible physical particles. Those physical particles were called atoms. The atomist theory was a pure philosophical speculation and had just a little of experimental support. The study of matter would require experiment going to very small distances which was impossible at the time. It also lacked strong theoretical foundations and therefore had no predictive power. Nevertheless, the atomist theory was an ingenious attempt to describe matter in terms of basic elementary constituents.

As time went by, new ideas and mathematically rigorous theories showed up. The technical advance allowed carrying on complicated experiments to test such ideas as well as derive new information about matter and interaction forces. Today, modern physics states that matter consist of stucturless fundamental units called elementary particles. Fundamental forces via which matter interacts arise from the exchange of such particles. Elementary particles and their interactions are studied by a branch of physics named particle physics. The current state of experimentation allows to perform studies at very small distances of about $10^{-18}$ meters. This requires acceleration of particles to a very high energy as compared to the masses of the particles involved. This is why very often particle physics is called high energy physics (HEP). The relationship between mass and energy is given by the famous Einstein's equation, $E=m c^{2}$. 
In particle physics, the theory that describes fundamental particles and their interaction is called the Standard Model (SM). It incorporates, in a single framework, all known elementary particles along with most of the major interaction types known to date. The interaction not included in the Standard Model is gravity. At the subatomic level, the effects of gravity are very small and thus can be neglected as compared to the other types of interaction.

The Standard Model not only explained the diversity of the particles known in the early 1970s, but also predicted new ones. For example, it predicted the $b$-quark that was observed in 1977, the $W$ and $Z$ bosons discovered in 1983, the tau neutrino that had been found in 2000. The heaviest fundamental particle, the top quark, whose existence is also predicted by the Standard Model, was observed in 1995. The last but yet unobserved fundamental particle is the Higgs boson.

The mechanism of particles acquiring masses is given by the Standard Model, and the Higgs boson plays a key role in this mechanism. The masses of the $W$ and $Z$ bosons are generated through spontaneous symmetry breaking. The simplest way to induce this breaking is the Higgs mechanism. The Higgs boson is a scalar particle predicted by the mechanism. The mass of the Higgs boson, however, is not specified. While the Standard Model does not predict the mass of the Higgs boson, it establishes a relationship between masses of the top quark, the $W$ boson and the Higgs boson. The relationship allows us to set an indirect constraint on the Higgs boson mass. The direct searches of Higgs conducted by CERN experiments indicates that the boson has to be a heavy object with the lower mass bound of $114 \mathrm{GeV}[1]$.

Although the SM predicts masses of the $W$ and $Z$ bosons, the masses of such particles as the bottom and top quark are not provided. The fermion masses are free parameters of the Standard Model and need to be determined experimentally. 
The knowledge of the top quark mass and mass of the $W$ boson not only limits the possible mass range of Higgs, but it also constitutes a consistency test of the Standard Model in many aspects. Early results of the top quark mass measurement showed that this quark is the most massive particle known. The purpose of the analysis presented in this thesis is to perform a precise measurement of the top quark mass in dilepton final states on the data from the $\mathrm{D} \varnothing$ experiment. Because of its heaviness, the top quark is a sensitive tool for studying the Higgs boson. It also may play a special role in the electroweak symmetry breaking of the Standard Model. Additionally, the precise measurement of the mass of the top quark also sets useful constraints on the theoretical models that extend the Standard Model. The analysis of this thesis is the most precise measurement of the top quark mass in the dilepton channel to the date. The achieved measurement uncertainty is $1.6 \%$. 


\section{Chapter 2}

\section{THEORETICAL OVERVIEW}

This chapter provides an overview of the Standard Model, and also details theoretical aspects of the top quark. The general discussion of the SM and its main components the electroweak interaction (EW), Higgs mechanism and electroweak symmetry breaking, quantum chromodynamics (QCD) - are presented in Section 2.1. Section 2.2 describes the physics of the top quark: its properties, production and decay modes, mass definition etc. The place of the top quark in the SM and beyond as well as the connection to the Higgs boson are also discussed in Section 2.2.

\subsection{The Standard Model}

The Standard Model is a modern theoretical framework to describe fundamental particles and their interactions. It consists of two theories that are based on quantum mechanics and special relativity. The SM combines three out of four fundamental interactions: electromagnetic, weak and strong. Gravitation has a very weak strength as compared to other forces. It does not have a substantial effect in high energy physics experiments.

According to the SM, matter comes in two classes - quarks and leptons. The primary difference between quarks and leptons is that quarks interact through the weak, electromagnetic and strong strong force, while leptons interact only by weak and electromagnetic force. For each quark and lepton, the SM predicts also its antiparticle that has the same mass but the opposite charge. To distinguish the particle and its antiparticle, a bar is usually placed over the particle symbol indicating that this is an antiparticle. 
The quarks and leptons are fermions, i.e. they have half a unit of intrinsic angular momentum, or spin. The forces are mediated by gauge bosons (photon $\gamma, W^{ \pm}, Z$, gluon g). The bosons have integer spin and are described by quantum gauge-field theories. The masses of particles in the SM are believed to arise from interaction with a special quantum field called the Higgs field. The quantum of this field is named the Higgs boson $(H)$ and may have been observed at the Tevatron and LHC [2], [3].

The leptons and quarks are organized in three generations. Across the generations, the quarks and leptons have all properties identical except for their masses and flavor quantum numbers. There are six flavors of quarks: up $(u)$, down $(d)$, charm $(c)$, strange $(s)$, top $(t)$ and bottom $(b)$. Similarly, there are six flavors of leptons: electron $(e)$ and electron neutrino $\left(\nu_{e}\right)$, muon $(\mu)$ and muon neutrino $\left(\nu_{\mu}\right)$, tau $(\tau)$ and tau neutrino $\left(\nu_{\tau}\right)$. The leptons and quarks split into generations, as illustrated in Figure 2.1. For example, electron, muon and tau are identical but have different mass and lepton flavor quantum number. There are three lepton flavors: electron $\left(L_{e}\right)$, muon $\left(L_{\mu}\right)$ and tau $\left(L_{\tau}\right)$. In all processes except neutrino mixing, lepton flavor is conserved quantum number. The lepton flavor and charge of all SM leptons are given in Table 2.1. The six flavors of quarks are characterized by charge $(Q)$, strangeness $(S)$, charm $(C)$, beauty $(B)$ and truth $(T)$. Analogously to the leptons, Table 2.2 provides a classification of all SM quarks.

At the most basic level, ordinary matter consists entirely of particles from the first generation, i.e. the $u, d$ quarks and electrons. Although neutrinos in the SM are considered massless, indirect experimental results indicate that they have small, but non-zero mass. For the most of high energy physics experiments, however, neutrinos can still be considered to be massless. The more massive particles from the second and third generations cannot serve as building blocks of stable matter. Due to their heaviness, they rapidly decay to the quarks and leptons from the first generation via weak interactions. 
Table 2.1. Charge and lepton flavor for three generations of leptons.

\begin{tabular}{c|cccc}
\hline \hline Lepton & $Q$ & $L_{e}$ & $L_{\mu}$ & $L_{\tau}$ \\
\hline \hline $1^{\text {st }}$ generation & & & & \\
$e$ & -1 & 1 & 0 & 0 \\
$\nu_{e}$ & 0 & 1 & 0 & 0 \\
\hline $2^{\text {nd }}$ generation & & & & \\
$\mu$ & -1 & 0 & 1 & 0 \\
$\nu_{\mu}$ & 0 & 0 & 1 & 0 \\
\hline $3^{\text {rd }}$ generation & & & & \\
$\tau$ & -1 & 0 & 0 & 1 \\
$\nu_{\tau}$ & 0 & 0 & 0 & 1 \\
\hline \hline
\end{tabular}

Table 2.2. Charge and flavor quantum numbers for three generations of quarks.

\begin{tabular}{c|ccccc}
\hline \hline Quark & $Q$ & $S$ & $C$ & $B$ & $T$ \\
\hline \hline $1^{\text {st }}$ generation & & & & & \\
$u$ & $2 / 3$ & 0 & 0 & 0 & 0 \\
$d$ & $-1 / 3$ & 0 & 0 & 0 & 0 \\
\hline $2^{\text {nd }}$ generation & & & & & \\
$c$ & $2 / 3$ & 0 & 1 & 0 & 0 \\
$s$ & $-1 / 3$ & -1 & 0 & 0 & 0 \\
\hline $3^{\text {rd }}$ generation & & & & & \\
$t$ & $2 / 3$ & 0 & 0 & 0 & 1 \\
$b$ & $-1 / 3$ & 0 & 0 & -1 & 0 \\
\hline \hline
\end{tabular}


The $u$ and $d$ quarks are often called light quarks. Sometimes, depending on the context, the $c$ and $s$ quarks can be also referred as light quarks. The $c$ and $s$ quarks are not massive if compared to $b$ and $t$.

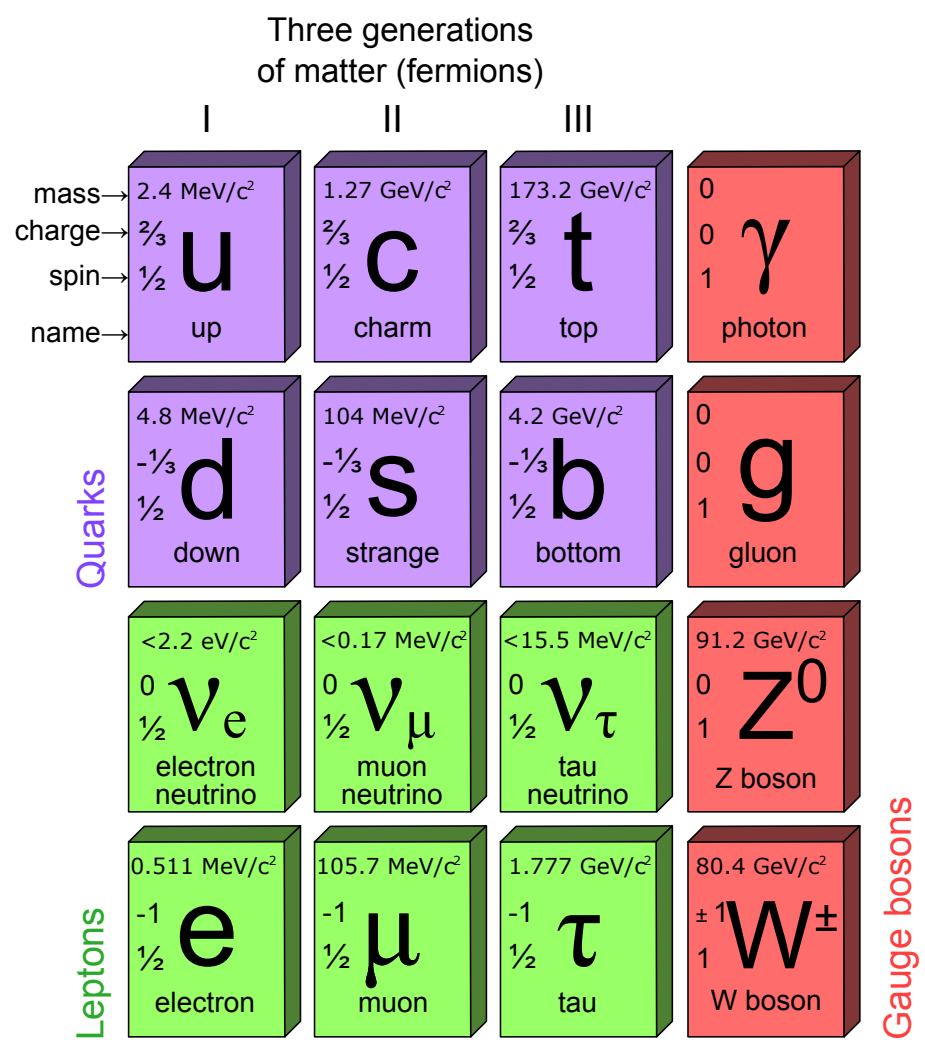

Figure 2.1. Fundamental particles of the Standard Model. Matter particles are the quarks and leptons. Force mediators are the gauge bosons.

Similarly to classical mechanics, the SM is described by a Lagrangian that is constructed from quantum fields. It is common in physics for symmetry to play a very important role, and this is also the case for the SM. According to Noether's theorem, if a Lagrangian is invariant under some symmetry transformation, there must exist some 
conserved quantity. Thus, for example, the space-time symmetry of special relativity leads to conservation of energy and momentum. The invariance of the SM Lagrangian under local gauge transformations results in such conserved quantities as color $(C)$, weak isospin $(L)$, and weak hypercharge $(Y)$. The standard approach describing symmetries is group theory. In the language of group theory, the SM Lagrangian is determined by local $S U(3)_{C} \times S U(2)_{L} \times U(1)_{Y}$ gauge invariance. The $S U(3)_{C}$ subgroup describes strong interactions between quarks and gluons. The $S U(2)_{L} \times U(1)_{Y}$ subgroup is responsible for describing electroweak interactions. The model of electroweak interactions was proposed by Weinberg [4] and Salam [5]. It unifies electromagnetic and weak interactions incorporating massive bosons $\left(W^{ \pm}, Z\right)$.

The SM gauge group is characterized by three coupling constants: $g_{1}=\sqrt{5 / 3} g^{\prime}$ for $U(1)_{Y}, g_{2}=g$ for $S U(2)_{L}$, and $g_{3}=g_{s}$ for $S U(3)_{C}$. The coupling constant defines the strength of the interaction, and at low energy the relationship between constants is $g_{3}>g_{2}>g_{1}$.

Table 2.3 lists the four fundamental interactions, their mediators and the particles the forces act on. All fundamental particles in the SM and their properties are shown in Figure 2.1.

If the coupling constant is small, the perturbation theory is employed to calculate interaction processes of the SM. The evolution of states is described using the $S$-matrix formalism suggested by Heisenberg [6]. A physical process is given as an expansion of the $S$-matrix by an infinite power series in the coupling constant. A small coupling constant assures us that the next order term is small as compared to the previous one. An approximate result is obtained by cutting off the series after several terms when the next correction term is considered very small and can be neglected. The terms of the perturbative $S$-matrix can be graphically visualized. Such a pictorial representation in momentum space is called a Feynman diagram, named in honor of its inventor 
Table 2.3. The fundamental interactions in nature. The strength of an interaction is given in relationship to the strong interaction which is taken as unity.

\begin{tabular}{c|cccc}
\hline \hline Interaction & Carrier & Interacting particles & Strength & Range $(\mathrm{m})$ \\
\hline \hline Strong & Gluons $(g)$ & Quarks & 1 & $10^{-15}$ \\
\hline $\begin{array}{c}\text { Electro- } \\
\text { magnetic }\end{array}$ & Photon $(\gamma)$ & $\begin{array}{c}\text { Electrically } \\
\text { charged }\end{array}$ & $10^{-2}$ & $\infty$ \\
\hline Weak & $W^{ \pm}, Z^{0}$ & Quarks, leptons & $10^{-6}$ & $10^{-17}$ \\
\hline Gravitation & Gravitons & Particles & $10^{-43}$ & $\infty$ \\
& (hypothetical $)$ & with $m>0$ & & \\
\hline \hline
\end{tabular}

Richard Feynman. An example of a Feynman diagram contributing to electron-positron scattering is shown in Figure 2.2.

The lowest order Feynman diagrams contain no loops, and are called tree-level diagrams. In a tree-level diagram, each vertex is connected to every over vertex by only one internal line. Higher order diagrams that do contain loops are also known as radiative corrections.

The minimal version of the SM has only 19 free parameters. Using that relatively small set of parameters, the SM successfully describes fundamental interactions and the elementary constituents of matter. Within its framework, it predicts the results for a wide range of high energy physics experiments. Despite its remarkable achievements, the SM still leaves many unresolved issues. For example, it predicts an infinite mass for the Higgs boson if computed beyond the tree level. Precise calculation of the Higgs boson mass requires calculation of radiative corrections. However, the corrections turn out to be quadratically divergent, and thus take the Higgs mass to infinity. This problem is known as hierarchy problem, and is not solved within the framework of the SM. 


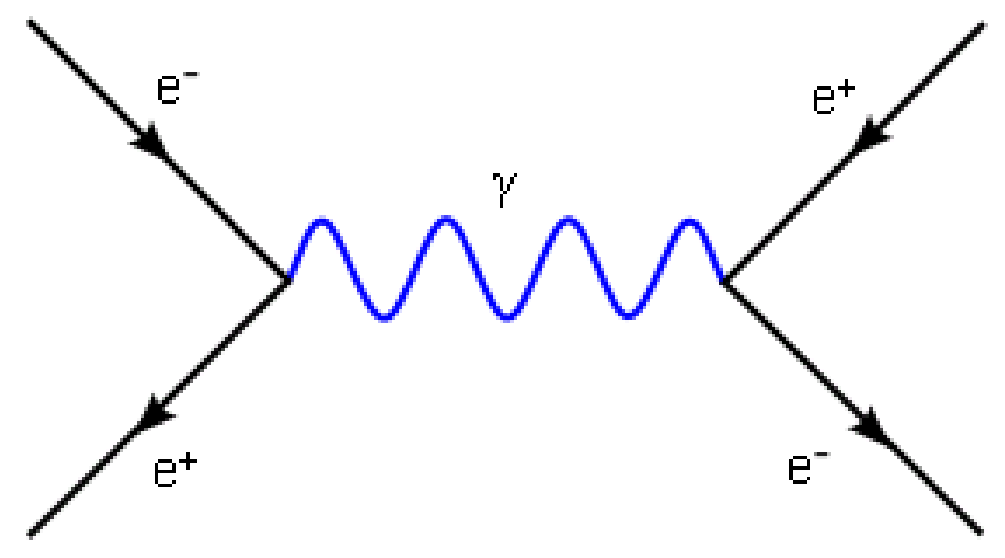

Figure 2.2. Second-order Feynman diagram representing $e^{+} e^{-}$pair annihilation to a virtual photon $\gamma$ with subsequent re-emission.

Although the SM is a successful theoretical approach in describing fundamental particles and interactions, it's obviously not a complete theory. The SM is believed to be the part of a more encompassing theory that is a joint description of all four fundamental interactions.

\subsubsection{The Electroweak Theory}

The Electroweak theory is a gauge theory that combines the electromagnetic and weak interactions. The development of the EW theory was one of the major achievements of 20th century physics. The description of the electroweak interaction is based on the $S U(2)_{L} \times U(1)_{Y}$ gauge symmetry group. The $S U(2)_{L}$ symmetry leads to conservation of weak isospin $L$ while $U(1)_{Y}$ results in hypercharge $Y$ conservation. At low energies, the electromagnetic and weak interactions differ significantly. They have different interaction ranges and interaction strengths. At high energies, however, they merge into a single electroweak interaction. Because the electromagnetic and weak forces be- 
come indistinguishable from each other, they are described within the single EW theory that unifies the interactions.

The part of EW theory that describes the electromagnetic interactions is called Quantum Electrodynamics (QED). Due to local $U(1)_{Y}$ gauge invariance, the electromagnetic interaction is mediated by only one gauge boson, the photon $(\gamma)$. The photon is a massless particle that couples to electrically charged particles. Because of its zero mass, the range of the electromagnetic interaction is infinite. The electromagnetic coupling constant equals $g_{1}=1 / 137$ in the region of low energies. This allows us to calculate QED processes with perturbation theory and Feynman diagrams. The small increase of the electromagnetic coupling constant with energy also assures us that the perturbative approach is valid in the high energy regions.

The $W^{ \pm}$and $Z$ are the gauge bosons of the weak force. The range of the weak interaction is found to be very small. This indicates that $W^{ \pm}$and $Z$ are very massive objects. As it is measured experimentally, masses of $W^{ \pm}$and $Z$ are $80.4 \mathrm{GeV}$ [7] and 91.2 $\mathrm{GeV}[8]$ respectively. Due to their large masses they quickly decay to other elementary particles and cannot be observed directly. Since $W^{ \pm}$are electrically charged they couple to a photon, and both $W^{ \pm}$and $Z$ couple to fundamental fermions which all carry a non-zero weak isospin.

The carriers of the weak interaction, $W^{ \pm}$and $Z$, are exactly analogous to the photon. Normally, they are also required to be massless and act like photons. This is, however, not consistent with the observations. The gauge invariance requires not only $W^{ \pm}$and $Z$ to be massless but also leads to the fermions having mass equal zero. The mathematical approach to generate particles masses is implemented through the idea of "spontaneous symmetry breaking". The spontaneous breaking of the gauge symmetry produces masses for the gauge bosons leaving electromagnetism as the only unbroken gauge symmetry. The result of the unbroken gauge symmetry in QED - a massless photon - is consistent 
with experiments.

\subsubsection{Spontaneous Symmetry Breaking and The Higgs Mechanism}

Each of the gauge bosons in the EW theory is represented by a vector field. There are three vector fields, $A_{\mu}^{i}(i=1,2,3)$ for $S U(2)_{L}$ part, and one $B_{\mu}$ for $U(1)_{Y}$. The requirement that the Lagrangian has to be gauge-invariant does not allow mass terms in the Lagrangian like $m^{2} A_{\mu} A^{\mu}$. Since the term $m^{2} A_{\mu} A^{\mu}$ is not gauge invariant, the carriers of gauge fields are required to be massless.

To generate masses for all EW gauge bosons except the photon, the theory is spontaneously broken via the Higgs mechanism. The theory is called spontaneously broken when the Lagrangian is invariant under gauge transformations but the vacuum state (i.e. the state of minimum energy) is not. For the $S U(2)_{L} \times U(1)_{Y}$ gauge symmetry, the simplest way to achieve spontaneous symmetry breaking is to introduce an isodoublet of complex scalar fields

$$
\phi(x)=\left(\begin{array}{c}
\phi^{+}(x) \\
\phi^{0}(x)
\end{array}\right)
$$

that has a non-zero vacuum expectation value $<\phi(x)>_{0} \neq 0$. The $\phi^{+}(x)$ and $\phi^{0}(x)$ in $\phi(x)$ are the charged and neutral scalar, respectively. The Lagrangian associated with the Higgs doublet can be written as follows

$$
\mathcal{L}=\left(D_{\mu} \phi(x)\right)^{\dagger} D^{\mu} \phi(x)+\mu^{2} \phi(x)^{\dagger} \phi(x)-\lambda\left(\phi(x)^{\dagger} \phi(x)\right)^{2}
$$

where $D_{\mu}=\left(\partial_{\mu}+i \frac{g}{2} \sigma_{i} A_{\mu}^{i}+i \frac{g^{\prime}}{2} B_{\mu}\right)$, and $\sigma_{i}$ are the Pauli matrices. The parameters $\mu$ and $\lambda$ satisfy $\mu^{2}<0$, and $\lambda>0$.

By assigning a non-zero vacuum expectation value 


$$
<\phi(x)>_{0}=<0|\phi(x)| 0>=\frac{1}{\sqrt{2}}\left(\begin{array}{l}
0 \\
v
\end{array}\right)
$$

where $v=\sqrt{\frac{\mu^{2}}{\lambda}}$, the ground state explicitly breaks $S U(2)_{L} \times U(1)_{Y}$ symmetry to $U(1)_{Q}$ of electromagnetism, leaving the photon massless. The perturbations around the ground state may be written as

$$
\phi(x)=\frac{1}{\sqrt{2}}\left(\begin{array}{c}
0 \\
v+h(x)
\end{array}\right)
$$

where $h(x)$ is the scalar Higgs field. The excitation of $h(x)$ is the Higgs boson. The deviation from vacuum is fully parameterized by Equation (2.4).

By substituting the vacuum expectation value into the Lagrangian from Equation (2.2), the masses generated for the gauge bosons can be determined

$$
\begin{gathered}
M_{W}=\frac{1}{2} g v \\
M_{Z}=\frac{1}{2} \sqrt{g^{2}+g^{\prime 2} v} \\
M_{A}=0
\end{gathered}
$$

with the physical states defined in terms of $A_{\mu}$ and $B_{\mu}$ as follows

$$
\begin{gathered}
W_{\mu}^{ \pm}=\frac{1}{\sqrt{2}}\left(A_{\mu}^{1} \mp i A_{\mu}^{2}\right) \\
Z_{\mu}=\frac{1}{\sqrt{g^{2}+g^{\prime 2}}}\left(g A_{\mu}^{3}-g^{\prime} B_{\mu}\right) \\
A_{\mu}=\frac{1}{\sqrt{g^{2}+g^{\prime 2}}}\left(g^{\prime} A_{\mu}^{3}+g B_{\mu}\right)
\end{gathered}
$$

Introducing the Weinberg mixing angle defined as $\tan \theta_{W}=\frac{g^{\prime}}{g}$, Equations (2.9) and (2.10) can be written as 


$$
\begin{aligned}
& Z_{\mu}=\cos \theta_{W} A_{\mu}^{3}-\sin \theta_{W} B_{\mu} \\
& A_{\mu}=\sin \theta_{W} A_{\mu}^{3}+\cos \theta_{W} B_{\mu}
\end{aligned}
$$

Using Equations (2.5) and (2.6), the relationship between masses of the $W$ and $Z$ bosons can be established as follows

$$
M_{Z}=\frac{M_{W}}{\cos \theta_{W}}
$$

The mixing angle is the only measurable parameter that allows us to probe the symmetry-breaking mechanism. The SM also provides the relationship between coupling constants, mixing angle and the electromagnetic charge $e$

$$
\begin{aligned}
& e=g \sin \theta_{W} \\
& e=g^{\prime} \cos \theta_{W}
\end{aligned}
$$

The value of the mixing angle can be determined, for instance, through measurement of a parity-violating asymmetry in scattering of longitudinally polarized electrons [9]. The measurement was performed on a fixed unpolarized targets yielding $\sin ^{2} \theta_{W}=0.2397 \pm 0.0010$ (stat.) \pm 0.0008 (syst.).

Knowing the $W$ mass [7] and the $\sin ^{2} \theta_{W}$ value, mass of the $Z$ boson can be calculated by Equation (2.13). The measured value of the $Z$ boson mass [8] is in good agreement with the theoretical prediction. The Higgs Mechanism therefore was validated when the $Z$ boson was discovered.

Using the relationship between the Fermi constant and the $W$ mass

$$
G_{F}=\frac{\sqrt{2} g^{2}}{8 M_{W}^{2}}
$$


the vacuum expectation value for the Higgs field can be obtained by inserting $M_{W}$ from Equation (2.5), giving

$$
v=\frac{1}{\left(\sqrt{2} G_{F}\right)^{1 / 2}}
$$

By plugging in the measured value of $G_{F}=1.6637 \cdot 10^{-5} \mathrm{GeV}^{-2}[10]$, the vacuum expectation value is found to be $v=246.22 \mathrm{GeV}$. Using Equations (2.16), (2.13), (2.14), $M_{W}$ and $M_{Z}$ can be expressed in terms of $G_{F}, e$, and $\theta_{W}$ as follows

$$
\begin{gathered}
M_{W}=\frac{e}{\sin \theta_{W}\left(\sqrt{32} G_{F}\right)^{1 / 2}} \\
M_{Z}=\frac{e}{\sin \left(2 \theta_{W}\right)\left(\sqrt{32} G_{F}\right)^{1 / 2}}
\end{gathered}
$$

Taking into the account the relationship between fine structure constant $\alpha$ and the electromagnetic charge, $\alpha=\frac{e^{2}}{4 \pi}, M_{W}$ and $M_{Z}$ can be expressed in fundamental physical constants only

$$
\begin{aligned}
& M_{W}=\frac{1}{\sin \theta_{W}}\left(\frac{\pi \alpha}{\sqrt{2} G_{F}}\right)^{1 / 2} \\
& M_{Z}=\frac{1}{\sin \left(2 \theta_{W}\right)}\left(\frac{\sqrt{8} \pi \alpha}{G_{F}}\right)^{1 / 2}
\end{aligned}
$$

The model also provides masses of fermions through their Yukawa coupling $y_{f}$ to the Higgs field

$$
m_{f}=\frac{y_{f}}{\sqrt{2}} v
$$

where $y_{f}$ is the Yukawa coupling. Since the fermion couplings are not known, the masses of fermions are parameters of the model. In addition to generating the fermion masses, the couplings produce mixing between mass eigenstates and the weak-interaction eigen- 
states. This allows for flavor-changing interactions and the fermions of heavy families may decay to the lighter fermions.

The mass of the Higgs boson is given through the unknown parameter $\lambda$

$$
M_{H}=\lambda v
$$

and therefore $M_{H}$ is a parameter of the model as well. All other particles that do not interact the Higgs field according to the SM - the photon and gluons - remain massless.

The process of acquiring masses by fermions can be described in analogy of light traversing matter. When light propagates through matter, it slows down due to the index of refraction. Similarly, particles that do not interact with the Higgs field are massless, and therefore propagate at the speed of light. In contrast, particle interacting with the Higgs field experience "a drag" and thus cannot propagate at the speed of light. The drag arising from interaction with the Higgs field is equivalent to the particle acquire a mass.

\subsubsection{Quantum Chromodynamics}

Quantum Chromodynamics (QCD) is the local gauge theory that describes strong interactions. The description of the strong interaction is based on the $S U(3)_{C}$ gauge symmetry group, which results in conservation of a quantum number called color charge, or just color. The color charge is a property of quarks and gluons. Particles of different color are experimentally indistinguishable. There are three color charges - red, green, or blue - and their anticolors - antired, antigreen, antiblue. The mediator of the strong interaction is a gluon. The gluon is a massless gauge boson that contains two color indices. Due to properties of the $S U(3)_{C}$ symmetry group, there are eight colored gluons. By exchanging gluons, the strong force is mediated between quarks that are also colored objects. Unlike the photons that do not carry electric charge, gluons carry a 
color charge and may interact with each other.

As opposed to QED, the strength of the strong interaction $g_{3}$ increases with increase in distance between interacting particles. The color-charged particles cannot be separated by a distance larger than the size of a hadron $\left(10^{-15} \mathrm{~m}\right)$. For this reason, quarks and gluons are never observed as free particles, but instead, they exist only inside composite colorless particles. This phenomenon is called color confinement, or just confinement. Such composite particles are called hadrons. There are two types of hadrons: mesons and baryons. The mesons are the bound-state of a quark and antiquark in such way that their color charges cancel. The baryons contain three quarks with the neutral, or "white", total color charge. For instance, the proton and neutron are baryons. Their bound states are $u$ ud and $u d d$ respectively. The $\pi^{+}$is a meson and made of $u \bar{d}$ combination. In nuclear physics, mesons serve as the carriers of the nuclear force that holds together protons and neutrons in a nucleus.

The fundamental property of QCD is asymptotic freedom. The idea of asymptotic freedom is that at large energies or very small distances, the strength of the strong interaction $g_{3}$ decreases. To the lowest order of calculations, it had been found [11] that

$$
g_{3}\left(Q^{2}\right)=\frac{1}{\beta_{0} \ln \left(Q^{2} / \Lambda_{Q C D}^{2}\right)}
$$

where $\beta_{0}$ is a quantity depending on the number of quarks that contribute to the calculation, $Q$ is the momentum transfer involved in the interaction, $\Lambda_{Q C D}^{2}$ is a constant reflecting some reference scale. The value is found experimentally $\Lambda_{Q C D}=200 \mathrm{MeV}$. The dependence of $g_{3}$ on $Q^{2}$ is called a running coupling constant. At large values of $Q^{2}$, $Q^{2} \gg \Lambda_{Q C D}^{2}$, the coupling constant is small and thus enables perturbative expansion in terms of $g_{3}$. For instance, at $Q^{2} \simeq M_{Z}^{2}$, the coupling constant is $g_{3}\left(M_{Z}^{2}\right)=0.12[12]$. The part of QCD that can be calculated using perturbation theory is sometimes referred as perturbative $Q C D$, or just $p Q C D$. As $Q^{2}$ is decreasing, the coupling constant grows - 
already at $Q^{2}=4 \mathrm{GeV}^{2}, g_{3}\left(Q^{2}\right)=0.32$. When $Q^{2}$ is of the order of $\Lambda_{Q C D}^{2}, Q^{2} \sim \Lambda_{Q C D}^{2}$, the coupling constant becomes very large, and calculations in series of $g_{3}$ are not possible any longer. Thus, $\Lambda_{Q C D}$ is a constant that sets the scale at which QCD becomes non-perturbative. It is also used to separate heavy quarks from light quarks. The heavy quarks satisfy the condition $m \gg \Lambda_{Q C D}$, where $m$ is the quark mass. At collider experiments, such as Tevatron, the momentum transfer is high and pQCD calculations can be employed. In the region of low energies, often referred as soft $Q C D$, the approach of pQCD is not applicable, and the calculations may be performed numerically using Lattice Field Theory in combination with Monte Carlo methods. In Lattice QCD, the calculations are performed at discrete space-time points on a lattice, and the results are obtained by extrapolating lattice spacing to zero.

The validity of QCD in the perturbative regime has been tested in many experiments. So asymptotic freedom justifies QCD parton model and the color charge that were originally introduced to describe wide variety of hadrons. The running coupling constant also explains asymptotic freedom at large momentum transfer and the parton confinement at small distances.

\subsection{The Top Quark}

Once the $\tau$ lepton had been found in 1976, a strong theoretical argument indicated the existence of a third generation of quarks. One of them was observed already in 1977 and is named the bottom quark, or the $b$-quark. To establish weak isospin state of the $b$ quark, the partial decay width of the $Z \rightarrow b \bar{b}$ process was derived from the measurements of $R_{b}=\Gamma_{b} / \Gamma_{h a d}[13]$ and $\Gamma_{h a d}[14]$. The derivation yields $\Gamma_{b}(Z \rightarrow b \bar{b})=388 \pm 13 \mathrm{MeV}$ and the result agrees with the $\mathrm{SM}$ prediction $\Gamma_{b}^{t h}(Z \rightarrow b \bar{b})=378 \pm 3 \mathrm{MeV}[15]$ assuming $L_{3}=-1 / 2$ for the weak isospin. This indicates the doublet state of the bottom quark and implies the existence of the $b$-quark weak isospin partner with $L_{3}=1 / 2$ that is called 
the top quark. The searches for the top quark started in the late 1970's and finished with its discovery in 1995 [16], [17].

The initial expectation for the top quark mass was about $30 \mathrm{GeV}$. However, the newly built Sp $\bar{p}$ S collider at CERN that ran until the mid-1980's ruled out this possibility. In 1993, the CDF experiment at Fermilab had set a lower limit on the mass at $91 \mathrm{GeV}$ [18]. The DØ experiment in 1994 excluded masses up to $131 \mathrm{GeV}$ [19]. The first measurement of the top quark after its discovery in 1995 was $m_{t}=176.0 \pm 8$ (stat.) \pm 10 (syst.) $\mathrm{GeV}$ by CDF [17] and $m_{t}=199.0_{-21}^{+19}$ (stat.) \pm 22 (syst.) GeV by D $\varnothing[16]$. The latest Tevatron average mass of the top quark that combines the results from the $\mathrm{D} \varnothing$ and CDF experiments is $m_{t}=173.1 \pm 1.3 \mathrm{GeV}[20]$.

Due to its massiveness, the top quark has an extremely short lifetime. An indirect constraint on the top quark lifetime $\tau_{t}$ can be established by measuring its total decay width $\Gamma_{t}$. The lifetime is inversely related to the width, i.e. $\tau_{t}=1 / \Gamma_{t}$. The latest measurement [21] from the $\mathrm{D} \varnothing$ experiment for a top mass of $172.5 \mathrm{GeV}$ provides $\Gamma_{t}=2.0$ $\mathrm{GeV}$ that translates into $\tau_{t}=3.29 \times 10^{-25} \mathrm{~s}$. The top quark lifetime is significantly shorter than the time needed for the quark to interact through the strong interaction and form a hadron such as $t \bar{t}$ meson. The typical time needed for the quark to form a hadron is $\tau_{\text {had }} \approx \Lambda_{Q C D}^{-1}$. Using the value of $\Lambda_{Q C D}$ from the previous section, it can be found to be that $\tau_{h a d}=5.23 \times 10^{-25} \mathrm{~s}$. As it can be seen, the top quark lifetime is almost two times shorter than the hadronization timescale.

The very short lifetime allows us to study the quark before hadronization occurs. For instance, it enables study of the spin correlation between the top and antitop quark when they are produced in pairs. The total spin of the top and antitop, as well as total spin of their decay products, in such a case is conserved.

The charge of the top quark predicted by the SM equals $2 e / 3$. The measurement of top quark charge [22] by the $\mathrm{D} \varnothing$ experiment shows that the observed charge is consistent 
with the SM prediction. The hypothesis of an existence of the exotic top quark with the charge $4 e / 3[23]$ is ruled out at the $92 \%$ confidence level.

The top quark is the only quark whose mass is of the order of the electroweak symmetry breaking scale, i.e. $v=246.22 \mathrm{GeV}$. The masses of all other fermions are smaller in the SM by at least two orders of magnitude and more. Using Equation (2.22), the Yukawa coupling of the top quark to the Higgs can be expressed as follows

$$
y_{t}=\frac{\sqrt{2} m_{t}}{v}
$$

By plugging in the numbers for the mass and vacuum expectation value, the coupling is found to be $y_{t}=0.996 \pm 0.008$. The fact that $y_{t}$ is very close to unity may indicate a fundamental role of the top quark in the electroweak symmetry breaking mechanism.

\subsubsection{Top Quark Production}

There are two production modes of the top quark: in pairs via strong interactions, and as a single quark via electroweak interactions. Top quarks pairs are produced either through quark-antiquark annihilation, or gluon-gluon fusion. The main leading-order (LO) Feynman diagrams for the strong interaction mode are shown in Figure 2.3.

To the leading-order of QCD calculations, the $t \bar{t}$ production results from quarkantiquark annihilation about $85 \%$ of the time; the remaining $15 \%$ comes from gluongluon fusion. The top quark production cross-section can be calculated using perturbative expansion. Recent theoretical calculations [24], [25] predict that for the top quark mass $m_{t}=171 \mathrm{GeV}$, an inclusive top quark pair production cross section at $\sqrt{s}=1.96$ $\mathrm{TeV}$ is $7.62 \mathrm{pb}$. A total uncertainty in the calculations is dominated by the PDFs and is estimated to be less than $10 \%$ for the NNLO calculations.

The EW production of a single top quark occurs either via the decay of the virtual $W$ boson to the top and bottom quarks in the $s$-channel, or via the exchange of the 


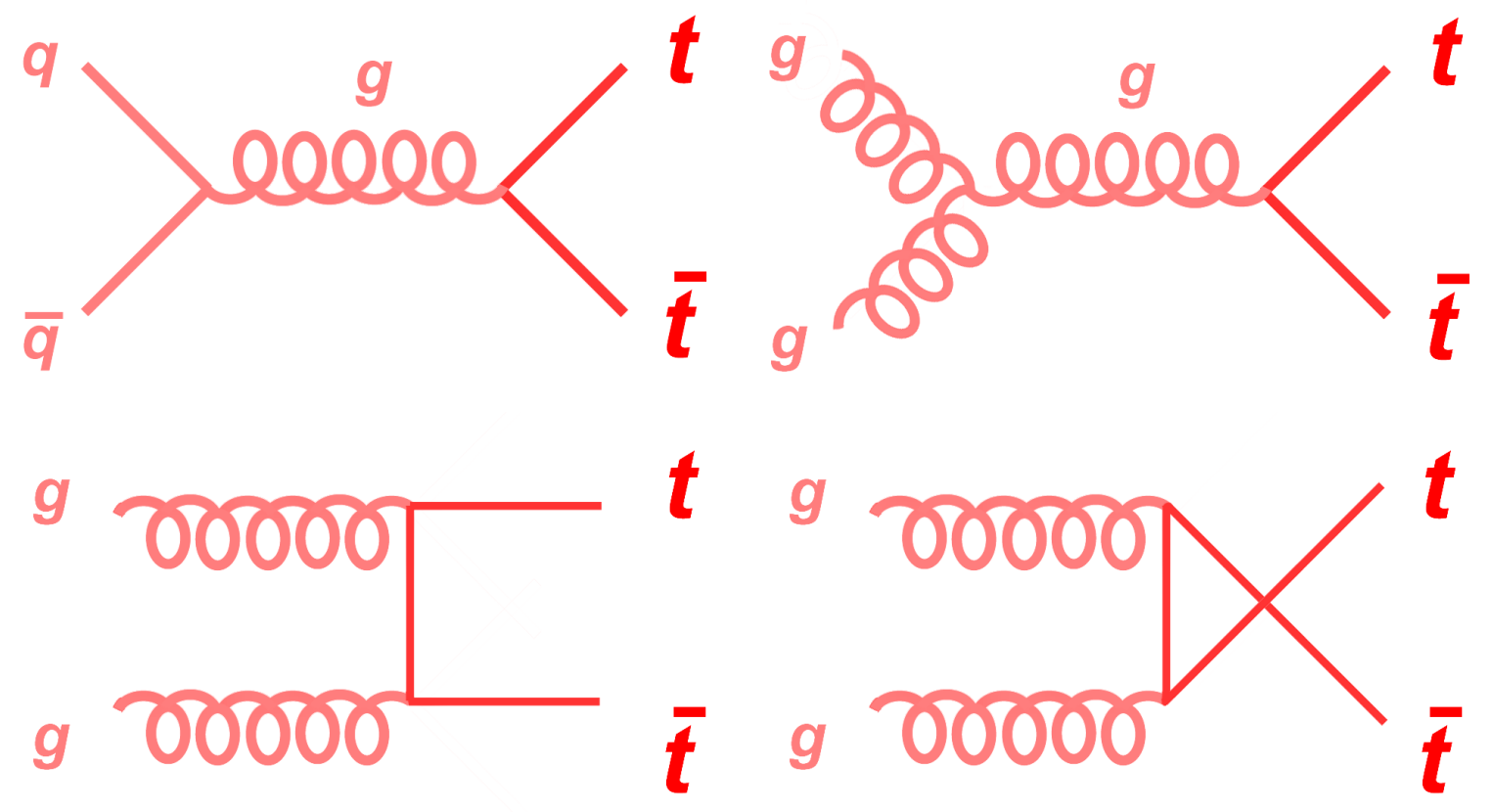

Figure 2.3. The leading order Feynman diagrams for $t \bar{t}$ production: quark-antiquark annihilation $(q \bar{q})$ and gluon-gluon fusion $(g g)$.

virtual $W$ between a light quark and a bottom quark in the $t$-channel. The leading order Feynman diagrams for the electroweak production mode are shown in Figure 2.4.

A third possible production channel for a single top proceeds via both the $s$-channel and $t$-channel in which the top quark is produced together with a $\mathrm{W}$ boson. Because of its very small cross section, this process is not considered.

The cross-section rate for the electroweak production mode at Tevatron is measured [26] to be $\sigma_{t}=3.43_{-0.74}^{+0.73} \mathrm{pb}$. Although the single top quark cross section is only about two times smaller than that for the top quark pair, the single top quark has an extremely large background contribution. The rate for the background processes are more than thirty times larger than that for the top quark pair events. In particular for this reason, the 

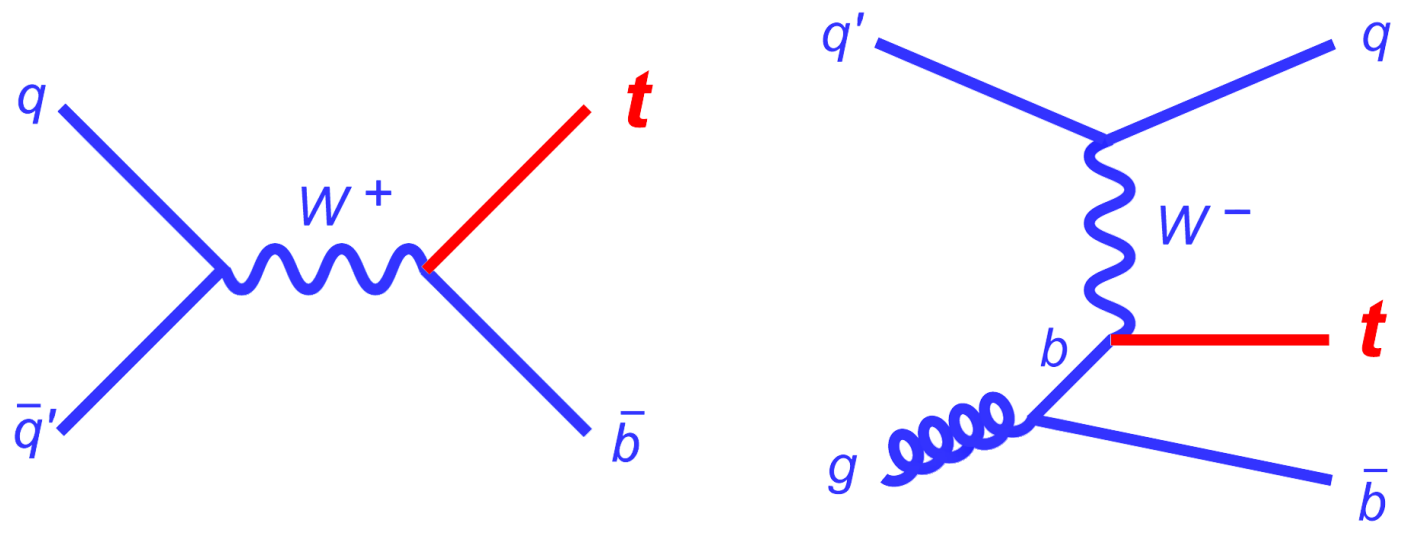

Figure 2.4. The leading-order Feynman diagrams for the electroweak production of a single top quark: $s$-channel (left), $t$-channel (right).

analysis of this thesis was performed on the $t \bar{t}$ events produced via the strong interactions.

\subsubsection{Top Quark Decay}

Due to the extremely short lifetime, the top quark decays so quickly that it can only be detected indirectly. An indirect observation of the top quark is performed via its decay products. According to the SM, most of the time the top quark decays through the electroweak interaction. In the electroweak model with one Higgs doublet, the top quark is expected to decay approximately $99.8 \%$ of the time to the $W$ boson and $b$-quark [12]. The decay to $s$ or even $d$-quark is allowed by the SM, but the probability for such

processes to occur is extremely small. Thus the process $t \rightarrow W^{+} b$ is the dominant decay mode. Analogously for the antitop, the dominant decay mode is $\bar{t} \rightarrow W^{-} \bar{b}$.

After the $W$ boson and $b$-quark are produced in the top quark decay, their further decay takes place. The $b$-quark hadronizes to a jet of the hadrons with one of the hadrons in the jet being a $b$-hadron. The $W$ boson decays into a lepton and neutrino, $W \rightarrow \ell \nu$, 
or to two quarks, $W \rightarrow q q^{\prime}$. The final states of the $t \bar{t}$ event are dictated by the possible decay modes of the $W$ boson. The branching ratios of the $W$ boson to various fermions define the relative rates of the $t \bar{t}$ final states. According to the decays of two $W$ bosons from the top quark pair, the events can be divided into the following final states:

- All hadronic. Both $W$ bosons decay to quarks $W \rightarrow q q^{\prime}$ that subsequently turn into jets of hadrons. Since the quark pairs coming from each $W$ can have three different combinations of color and anti-color, the all-hadronic final state branching ratio is much higher than the others, accounting for about $46 \%$ of $t \bar{t}$ events. Besides a high uncertainty on the measured energy of a jet, the channel also suffers from large multijet QCD background. The all-hadronic events are characterized by four energetic jets. The channel decay reaction can be written as $t \bar{t} \rightarrow q \overline{q^{\prime}}+q^{\prime \prime} q^{\prime \prime \prime}+b \bar{b}$.

- $\ell+$ jets. One of the $W$ bosons decays to lepton and neutrino, $W \rightarrow \ell \nu$, and the other into two quarks, $W \rightarrow q q^{\prime}$. The lepton $\ell$ can be an electron $e$ or a muon $\mu$. The decay mode, $W \rightarrow \tau \nu \rightarrow e(\mu) \nu \bar{\nu}$, cannot be distinguished from the direct $W$ decay to $e$ or $\mu, W \rightarrow e(\mu) \nu$. This case is therefore also included. The $\ell+$ jets channel is characterized by an isolated high-energy lepton, four energetic jets and a resulting large momentum imbalance due to an undetected neutrino. The $\ell+$ jets channel appears in $45 \%$ of $t \bar{t}$ events. The channel has modest background, primarily from $W+$ jets and QCD multijets events, and large signal statistics. The kinematic parameters of an undetected neutrino can be fully reconstructed to within a quadratic ambiguity from the measured kinematic quantities of other objects in the $\ell+$ jets event. The channel decay reaction can be written as $t \bar{t} \rightarrow$ $\ell \bar{\nu}+q \overline{q^{\prime}}+b \bar{b}$

- Dilepton. Both $W$ bosons decay leptonically $W \rightarrow \ell \nu$ producing a pair of leptons in the final state. The pair of leptons can be $e e, e \mu$, or $\mu \mu$. Similarly to the $\ell+$ jets channel, the $W$ decay mode, $W \rightarrow \tau \nu \rightarrow e(\mu) \nu \bar{\nu}$, is also included. 
The dilepton channel is characterized by two isolated leptons with large trasverse momentum, two or more energetic jets and large momentum imbalance. Unlike the $\ell+$ jets channel, the dilepton event cannot be uniquely reconstructed from the measured kinematic properties of the other event objects. The channel has a very distinct signal signature and low contamination from the background. The dilepton channel accounts approximately $9 \%$ of $t \bar{t}$ events. Although the dilepton mode suffers from low signal statistics, its final state contains isolated high-energy leptons with precisely reconstructed kinematic parameters that can be used in the mass analysis. The channel decay reaction can be written as $t \bar{t} \rightarrow \ell \overline{\ell^{\prime}}+\bar{\nu} \nu^{\prime}+b \bar{b}$.

\section{Top Pair Branching Fractions}

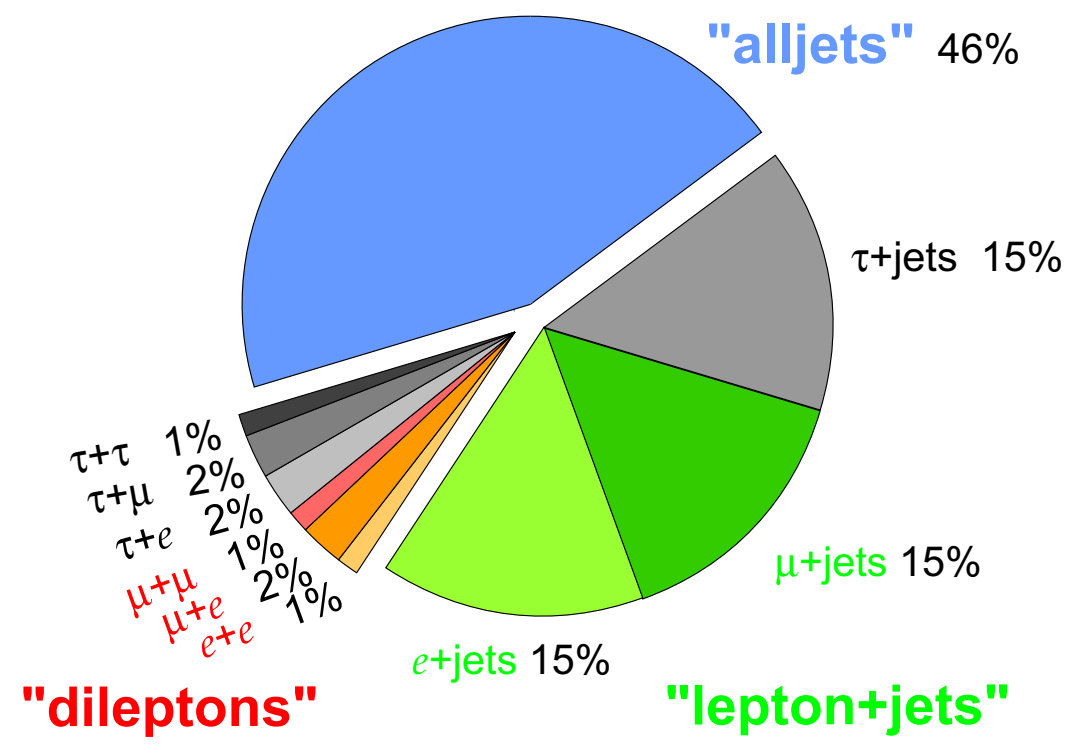

Figure 2.5. The $t \bar{t}$ decay modes and their respective branching ratios.

The branching ratio for each individual of $t \bar{t}$ decay is given in Figure 2.5. 


\subsubsection{The Definition of Mass}

The mass of a particle is one of the fundamental parameters in the SM. Nevertheless its definition is often non-trivial, especially for the quarks. Unlike many other fundamental particles, quarks are never observed as free particles. Instead, quarks are confined in mesons and baryons. The exception is the top quark that decays before hadronization occurs. The mass of a quark is usually defined by a certain physical concept that incorporates quark influence on hadronic properties. Hence the definition of mass is a complex one and has to be referenced to the particular theoretical prescription.

While computing values for physical quantities such as scattering amplitudes, a special factor called a Feynman propagator plays an important role. Thus, according to the Feynman rules, for each internal line, a factor

$$
f(q)=\frac{i}{q^{2}-m^{2}+i \varepsilon}
$$

is assigned, where $q$ is the four-momentum $q=(E, \vec{p})$ carried by the line in the diagram. The parameter $\varepsilon$ is an infinitesimally small positive quantity that is usually taken to zero at the end of the calculations. Equation (2.26) represents the Feynman propagator in the momentum space for the scalar particle.

One of the mass definitions is called the pole mass and related to the position of the divergence of the propagator. The pole mass is defined as the real part of the pole in the top quark propagator. When the denominator of the propagator $f(q)$ vanishes, i.e. $q^{2}=m^{2}$, this leads to the mass-shell condition. The mass-shell condition corresponds to a free particle with momentum $\vec{p}$ and energy $E$, and satisfies the relativistic equation $E^{2}=\vec{p}^{2}+m^{2}$. In this case, the parameter $m$ is the pole mass of the particle $m_{Q}$. The pole mass seemed to be the most natural perturbative definition of the quark mass.

When calculations are performed at the higher orders and loop diagrams are included, the perturbative corrections may become infinite. To avoid those infinities and 
make physical quantities finite, a special procedure called renormalization is employed. Renormalization is a particular subtraction approach that allows to absorb the infinities from the perturbative calculations. The most commonly used renormalization approach for pQCD is called the modified Minimal Subtraction scheme, or $\overline{M S}$ scheme. While removing the infinities, the renormalization also redefines the mass of the particles. In general, the mass parameter depends on the renormalization scheme used as well as on the renormalization scale parameter $\mu_{R}$. After all the infinities are removed by the subtraction and mass is redefined, the behavior near the mass-shell point is similar to that in the pole scheme, i.e. $\frac{i}{q^{2}-\bar{m}_{Q}^{2}\left(\bar{m}_{Q}^{2}\right)}$, where $\bar{m}_{Q}\left(\bar{m}_{Q}^{2}\right)$ is the redefined the $\overline{M S}$ mass parameter [27], [28] evaluated at a scale equal to the mass. The choice of $\overline{M S}$ mass is often more plausible for radiative corrections calculations.

The relationship between the pole mass $m_{Q}$ and the $\overline{M S}$ mass $\bar{m}_{Q}\left(\bar{m}_{Q}^{2}\right)$ is known up to the three loop level. To second order in $\alpha_{s}$, the pole mass and $\overline{M S}$ mass are related as follows

$$
m_{Q}=\bar{m}_{Q}\left(\bar{m}_{Q}^{2}\right)\left(1+\frac{4 \alpha_{s}\left(\bar{m}_{Q}^{2}\right)}{3 \pi}\right)
$$

where $\alpha_{s}\left(\bar{m}_{Q}^{2}\right)$ is the strong interaction coupling constants in the $\overline{M S}$ scheme evaluated at mass scale $\bar{m}_{Q}^{2}$.

For heavy quarks, the mass results are given either as the pole mass, or as the $\overline{M S}$ mass. In the analysis of this thesis, theoretical predictions are performed in the pole mass scheme, and hence the result is quoted as the pole mass.

\subsubsection{Top Quark in SM and beyond SM}

The mass of the Higgs boson is not predicted by the Standard Model. However, precise measurement of the top quark and $W$ boson mass allow us to set a powerful constraint on the Higgs boson mass. Within the SM, the masses are related by radiative 
corrections to the mass of the $W$ boson. The next-to-leading-order (NLO) Feynman diagrams, or radiative corrections to the $M_{W}$, are shown in Figure 2.6.
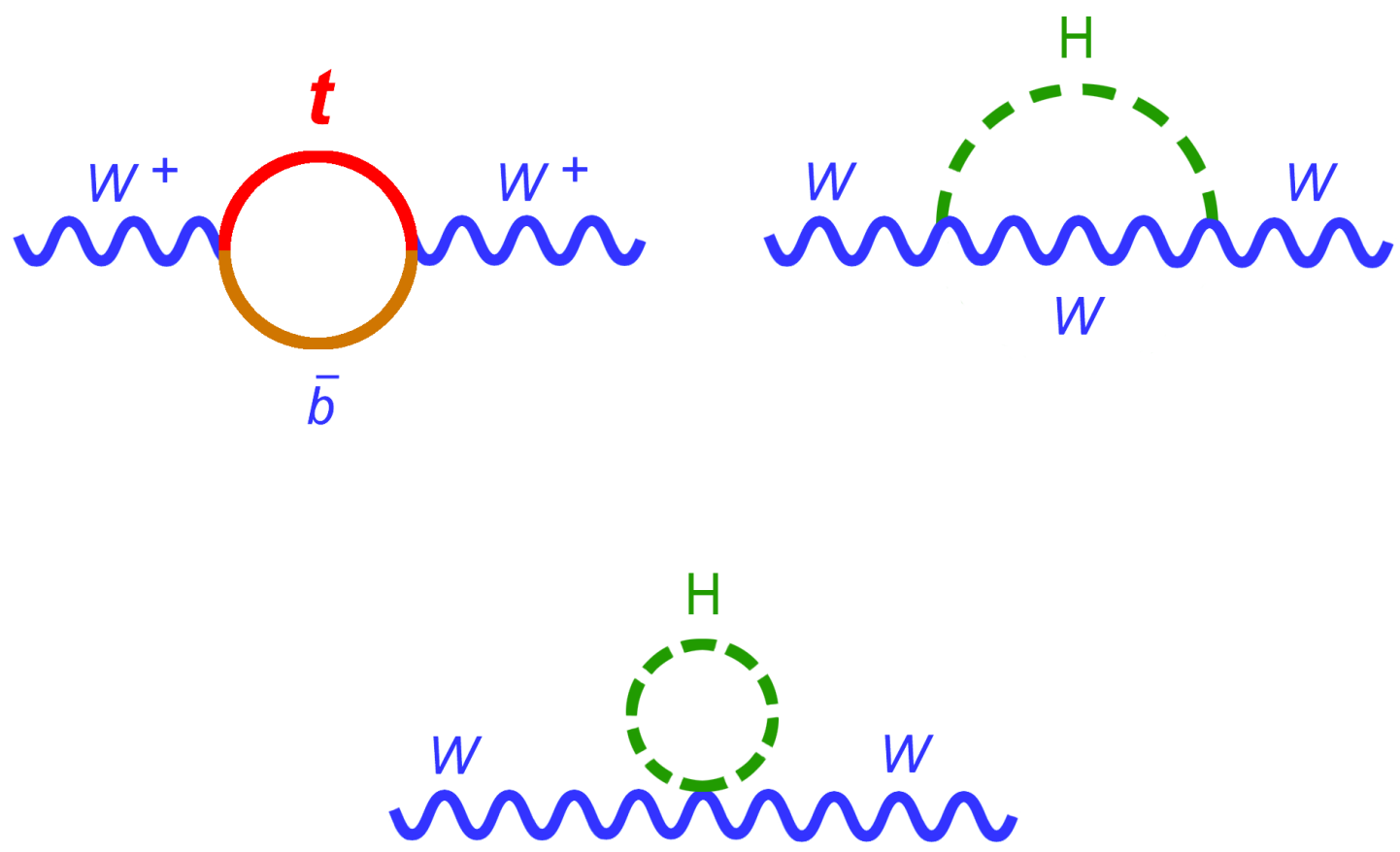

Figure 2.6. Representative Feynman diagrams for the radiation corrections.

The mass of the $W$ boson in Equation (2.20) is computed at tree level. At one-loop level, the mass of the $W$ boson acquires a finite radiative corrections, $\Delta r$, as follows

$$
M_{W}=\frac{1}{\sin \theta_{W}}\left(\frac{\pi \alpha}{\sqrt{2} G_{F}(1-\Delta r)}\right)^{1 / 2}
$$

Up to the two loop diagrams, the correction $\Delta r$ is given [29] as

$$
\Delta r=\frac{\alpha}{\pi \sin ^{2} \theta_{W}}\left(-\frac{3}{16 \sin ^{2} \theta_{W}} \frac{m_{t}^{2}}{m_{W}^{2}}+\frac{11}{48} \ln \frac{m_{H}^{2}}{m_{Z}^{2}}\right)+0.07
$$


As it can be seen, $\Delta r$ depends quadratically on $m_{t}$ and logarithmically on $m_{H}$. By plugging in Equation (2.29) to (2.28), it is possible to derive the relationship between $m_{W}, m_{t}, m_{Z}, \alpha, \sin \theta_{W}$ and $m_{H}$. Given the values for $\alpha, \sin \theta_{W}$ and $m_{Z}$, precise measurement of $m_{W}$ and $m_{t}$ imposes the constraint on the mass of the Higgs boson, $m_{H}$. Figure 2.7 from Ref. [30] shows the mass of the $W$ boson versus mass of the top quark. The blue ellipse indicates the constraints on $m_{W}$ and $m_{t}$ at $68 \%$ confidence level based on the data from Tevatron and LEP-II as of 2009. The dashed red contour is the constraint set by LEP-I and SLD. Green bands represent the theoretical constraint from the SM obtained for a region of the Higgs mass. The Higgs mass $m_{H}>1000 \mathrm{GeV}$ is not favored by the SM and thus is excluded theoretically. The ranges with $m_{H}<114 \mathrm{GeV}$, and $158<m_{H}<175 \mathrm{GeV}$ are excluded by direct Higgs searches.

The recent Tevatron combination of the top quark mass $m_{t}=173.1 \pm 1.3 \mathrm{GeV}[20]$ and the world average mass of the $W$ boson $m_{W}=80.399 \pm 0.023 \mathrm{GeV}[31]$ are consistent within the Standard Model framework. The results also indicate that the Higgs boson is likely to have a small mass. A better precision of the top quark and $W$ mass would provide us a better constraint on the Higgs boson mass.

In addition to setting a constraint on the Higgs mass, the precise knowledge of $m_{t}$ is important for testing QCD $t \bar{t}$ production. The $t \bar{t}$ cross section strongly depends on $m_{t}$. Disagreement between the measured cross section and theoretical prediction can be a sign of contributions from outside the SM. If the measured cross section is higher than the SM prediction, it may indicate a special role of the top quark in the electroweak symmetry breaking mechanism, such as in [32], [33].

In such extensions, the scalar Higgs field is removed in favor of new interactions that provide the observed mass spectrum. The symmetry breakdown occurs as a dynamical mechanism involving the top quark. For instance in the top condensation mechanism[32], the electroweak symmetry breaking arises from a natural top quark resonance, or conden- 


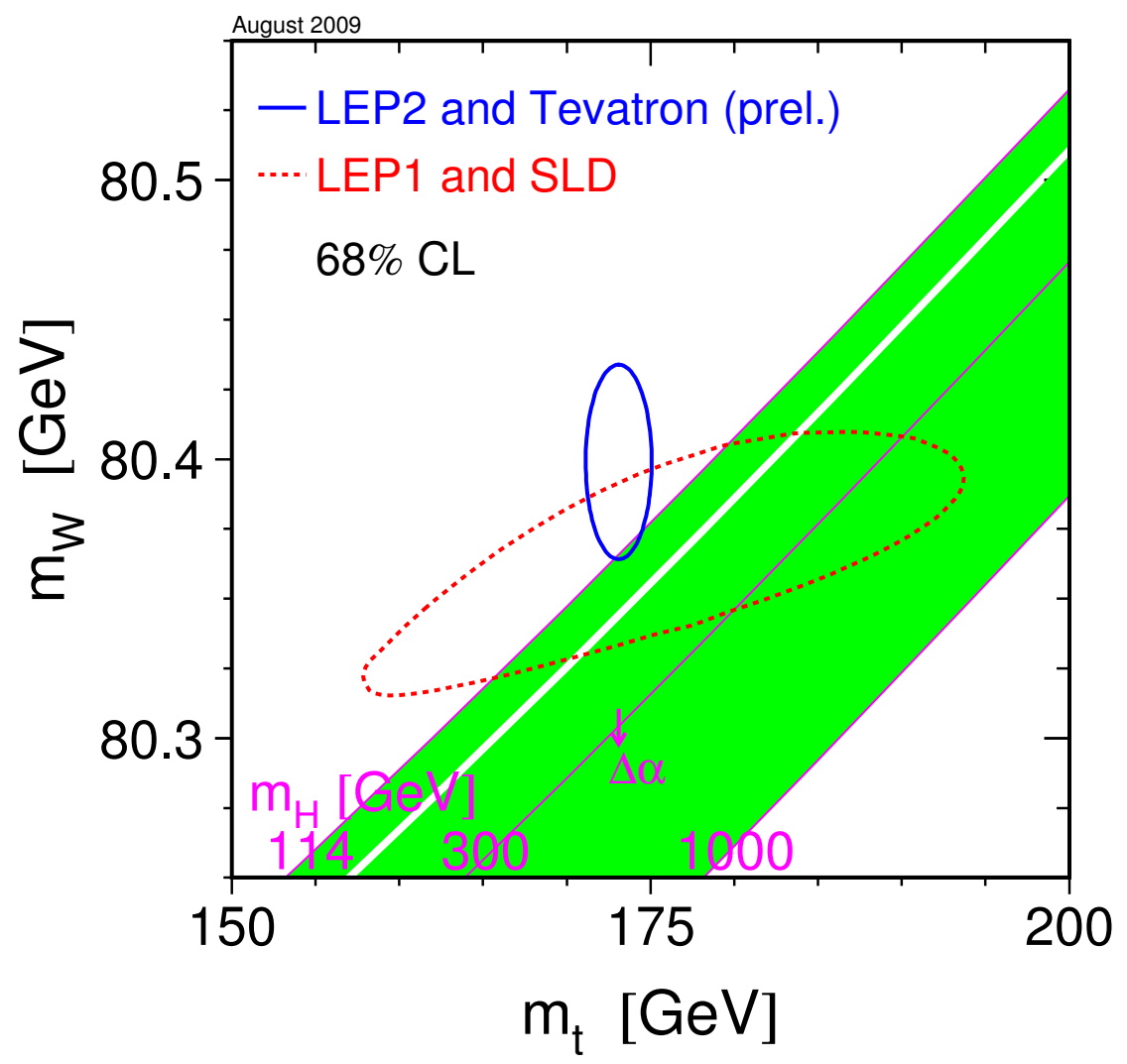

Figure 2.7. The indirect constraints on $m_{t}$ and $m_{W}$ (dashed red contour) from LEP-I and SLD data, and the direct measurements based on LEP-II and Tevatron data (solid blue ellipse) as of summer 2009. Green bands are the SM relationship for the masses as a function of the Higgs mass not excluded by the direct searches $\left(114<m_{H}<158 \mathrm{GeV}\right.$ and $\left.175<m_{H}<1000 \mathrm{GeV}\right)$. 
sate. The top-antitop pair acquires a vacuum expectation value similarly to the electron pairing condensate in superconductivity. Another example could be the topcolor-assisted technicolor model [34]. This model involves a $t \bar{t}$ condensate coming from new strong interactions causing the mesonic binding.

The recent cross section measurement in dilepton [35] and $\ell+$ jets [36] channels agrees with the Standard Model expectation. Figure 2.8 shows the experimental [36] and theoretical [37]-[42] cross sections as a function of the top quark mass. Hence, an accurate determination of the top quarks properties such as the top quark mass is of great importance since it allows to set a constraint on the Higgs mass, and to see if any hint of new physics may be visible. 


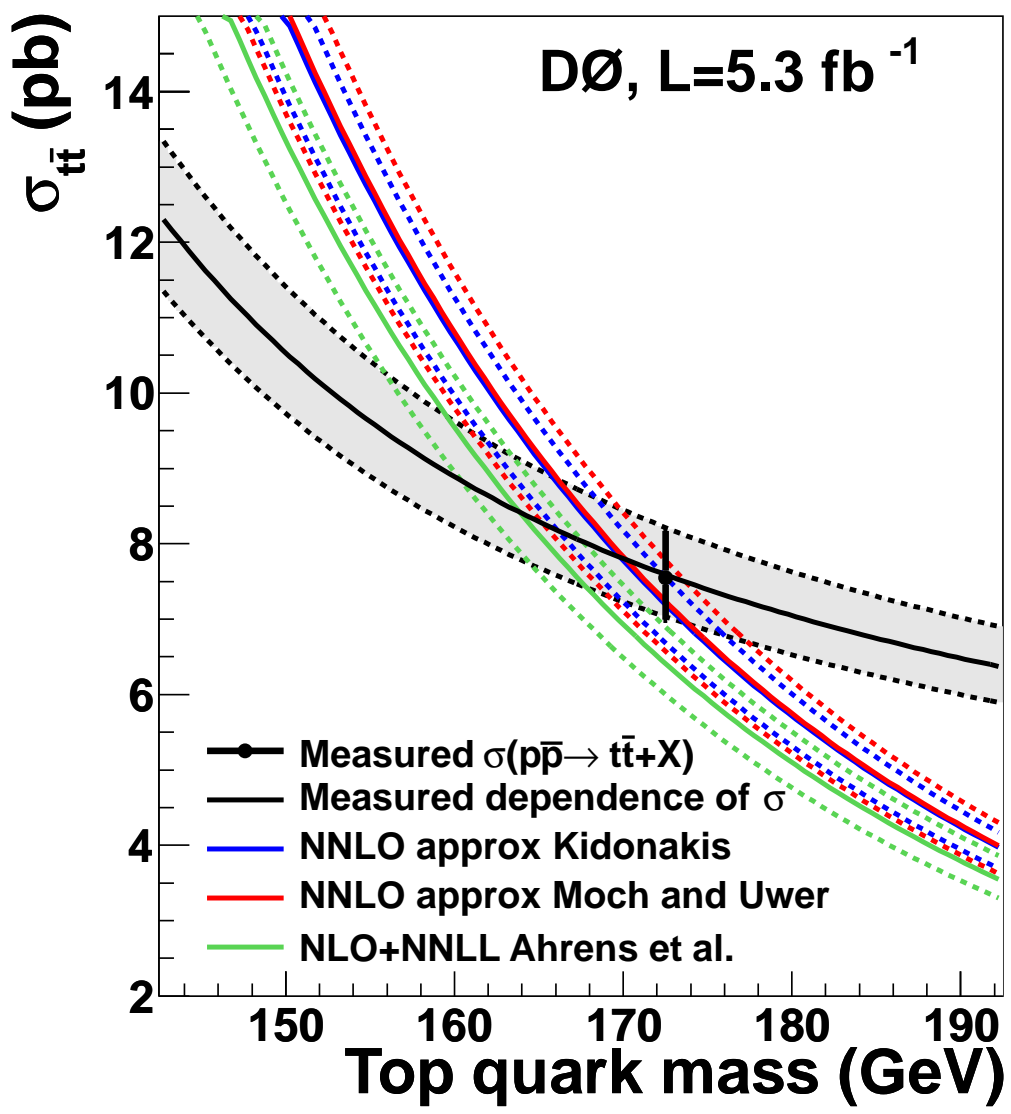

Figure 2.8. Experimental and theoretical $t \bar{t}$ cross section $\sigma_{t \bar{t}}$ as a function of the top quark mass $m_{t}$. The colored dashed lines represent the uncertainties for the theoretical predictions from the choice of the PDF, the renormalization and factorization scales. The point is the measured combined $\sigma_{t \bar{t}}$ evaluated for $m_{t}=172.5 \mathrm{GeV}$. The black curve shows experimental $\sigma_{t \bar{t}}$ as a function of $m_{t}$. The gray band corresponds to the total experimental uncertainty 


\section{Chapter 3}

\section{THE EXPERIMENTAL SETUP}

The analysis presented in this thesis has been performed with data collected by the DØ experiment at the Tevatron collider. This chapter gives an overview of the Tevatron accelerator complex and the $\mathrm{D} \varnothing$ detector.

\subsection{The Tevatron Collider}

The Tevatron [43] is a proton-antiproton $(p \bar{p})$ collider located at the Fermi National Accelerator Laboratory (Fermilab) near Chicago, Illinois. It was the highest energy particle accelerator until March 2010 with center-of-mass energy of $1.96 \mathrm{TeV}$. This energy is sufficient to produce the top quark. It remains one of only two places on Earth where the top quark can be produced artificially. The accelerator consists of a series of components that allow the production and the gradual acceleration of protons and antiprotons to the energy of $0.98 \mathrm{TeV}$. Subsequent collision happens at specific regions called interaction points. Two particle detectors, DØ and CDF, are located at and surround respective interaction points of the Tevatron ring. The particle collisions and consequent interaction are studied in detail at the detectors.

Several stages are required to accelerate particles and bring them into collision. Those include particle creation, cooling and acceleration. Each component of the Tevatron fulfills a specific role in particle production and acceleration. The schematic arrangement of the Tevatron components is shown in Figure 3.1. The main ones are: Cockcroft-Walton pre-accelerator, Linear Accelerator (Linac), Booster, Main Injector and the Tevatron ring. The following subsections describe these components in more detail. 


\section{FERMILAB'S ACCELERATOR CHAIN}

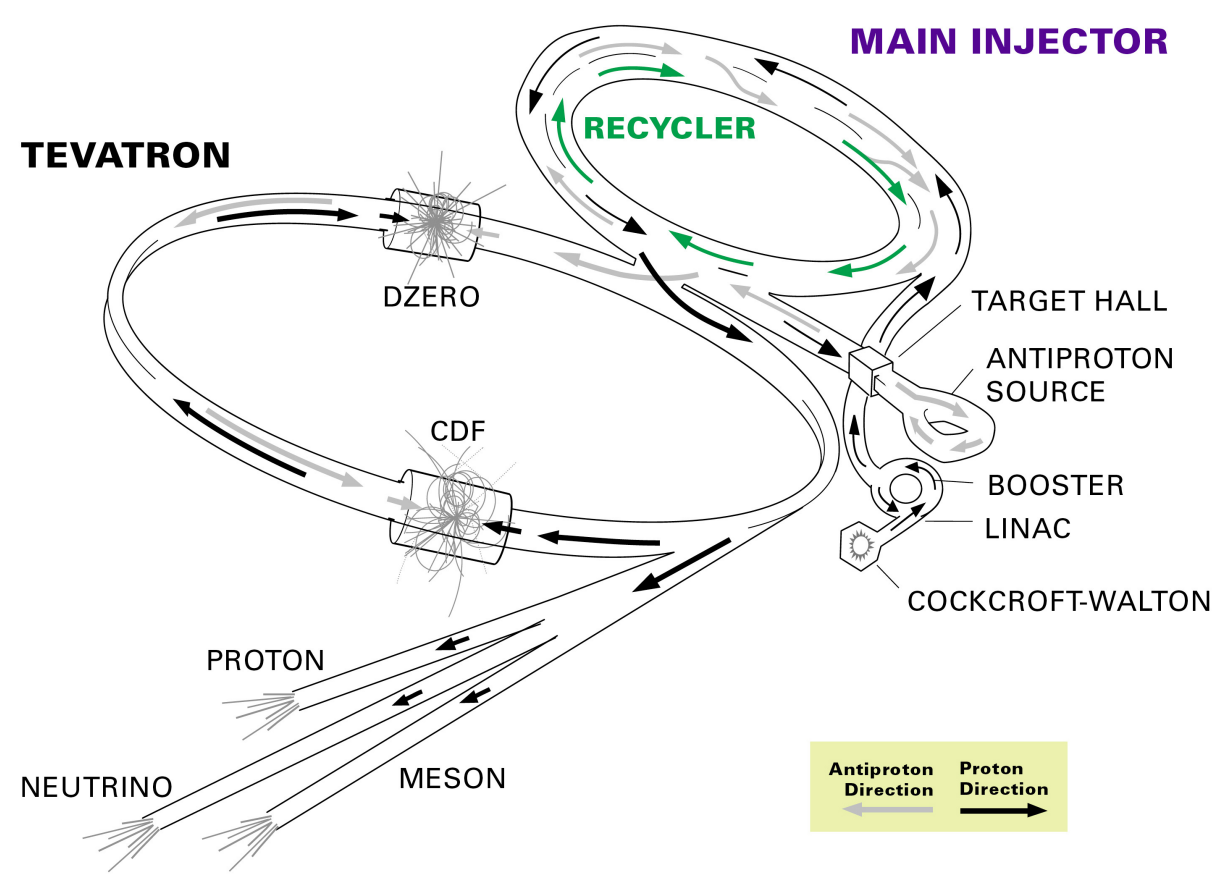

Figure 3.1. Tevatron Accelerator Complex. Many steps are required to bring into collision proton and antiproton beams. The locations of the D $\varnothing$ and CDF detectors are indicated. 


\subsubsection{Proton Creation and Acceleration}

The protons used in the Tevatron are derived from a source of negative hydrogen ions $\left(H^{-}\right)$that are produced in the magnetron [44]. The magnetron consists of an oval-shape cathode and a surrounding anode. It operates in a uniform magnetic field. To derive the ions, hydrogen gas under high pressure is pumped into the magnetron and an alternating pulse of a few hundred volts is applied at the rate of $15 \mathrm{~Hz}$. This produces a plasma of electrons and protons in the gap between the anode and cathode. As protons strike the cathode, they may pick up two electrons from the surface of the cathode and become $H^{-}$ions. Ultimately some $H^{-}$ions pass by an anode aperture and are accelerated to 18 $\mathrm{kV}$ by the extractor electrode to a Cockcroft-Walton generator. The schematic view of the magnetron is shown in Figure 3.2.

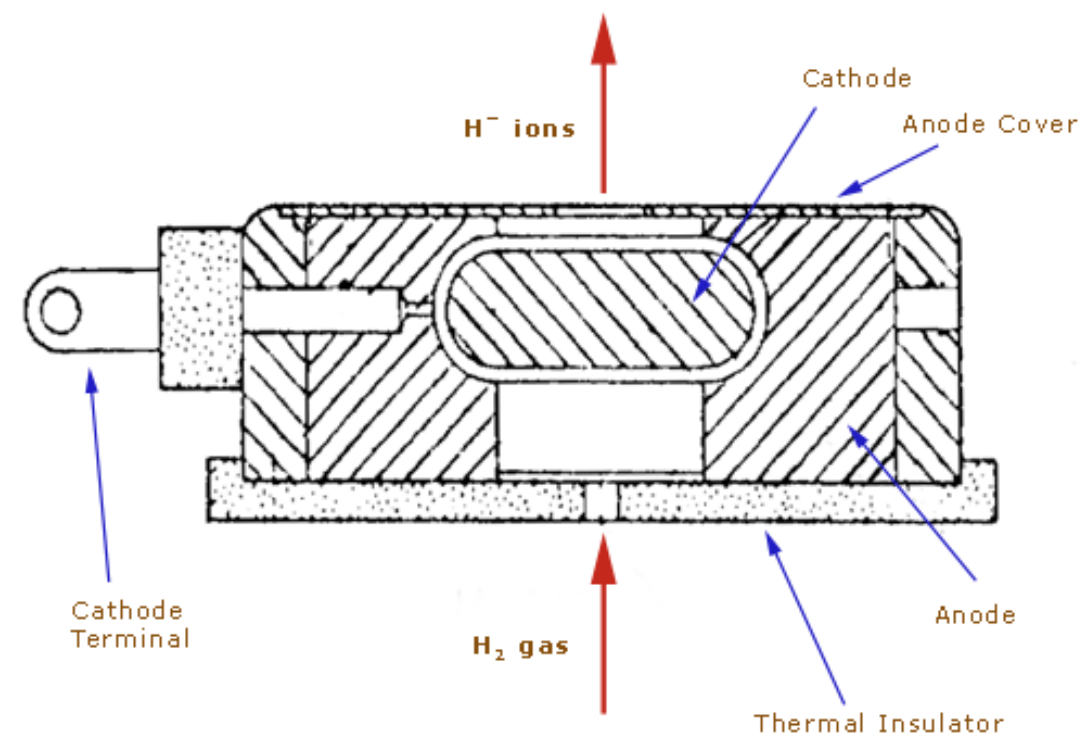

Figure 3.2. Schematic view of the magnetron, the $H^{-}$ion source used at Fermilab. 
At the Cockcroft-Walton generator, or multiplier, $H^{-}$ions are pre-accelerated in a $750 \mathrm{kV}$ static electric field. The Cockcroft-Walton generator is a special electric circuit that is constructed from diodes and capacitors to generate a high DC voltage from a low voltage $\mathrm{AC}$ input. An example of a two cascade multiplier that converts AC voltage input to two times higher DC output is shown in Figure 3.3.

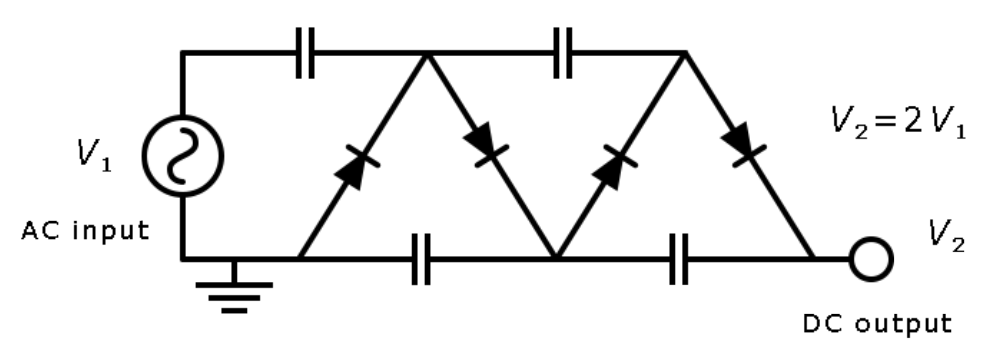

Figure 3.3. Electric circuit of the two cascade Cockcroft-Walton generator.

The static electric field produced in the multiplier accelerates particles before they go to the next accelerator. Unlike transformers, the multiplier does not require the presence of a heavy core or bulk insulator. The Cockcroft-Walton generator at Fermilab accelerates the ions to an energy of $750 \mathrm{keV}$ and is shown in Figure 3.4.

After the Cockcroft-Walton multiplier, $H^{-}$ions enter the Linac [45] for further acceleration to an energy of $400 \mathrm{MeV}$. The Linac is a linear accelerator that is 150 meters long and is composed from a number of radio-frequency (RF) cavities, or resonators, connected sequentially. Every cavity is made of a number of cells and strung together to form a resonator. A repetitive variation of the electric field inside of every cell at radio frequency propels the ions through one cell to another until the beam leaves with the desired energy. Figure 3.5 shows an example of such a cavity designed for future experiments such as Project X [46]. The Linac allows ion acceleration to much higher 


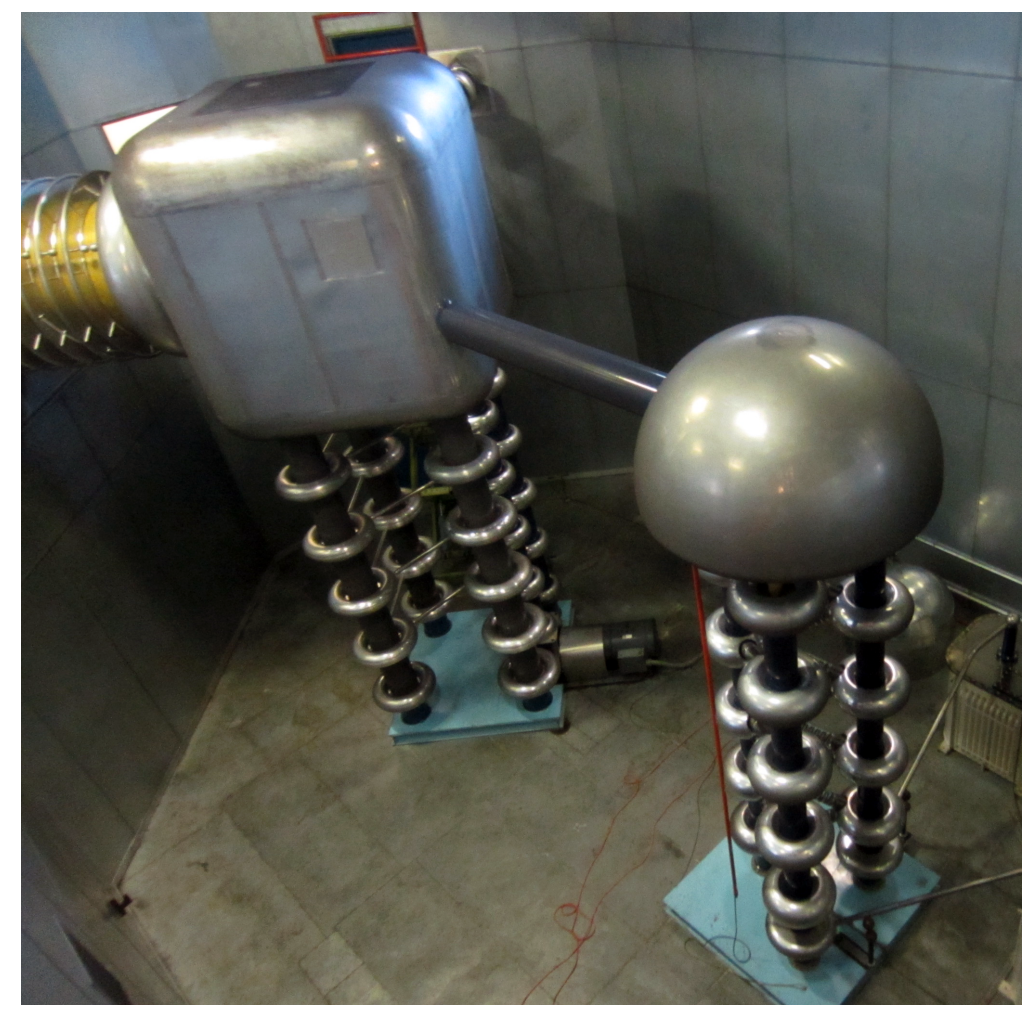

Figure 3.4. The picture of the Cockcroft-Walton pre-accelerator at Fermilab.

energy in a relatively short distance.

Once accelerated by the Linac, the ions are transferred into the Booster [47] passing through a thin carbon foil. The foil strips the electrons thus producing $\mathrm{H}^{+}$ions from $H^{-}$. The Booster is a circular accelerator 152 meters in diameter, and is the first synchrotron in the accelerator chain. It consists of a circular beam-pipe surrounded by non-superconducting magnets. The carbon foil is used to strip off the electrons and convert the ions into bare protons. In a synchrotron, the magnetic field is used to bend the beam in a pipe, and the electric field makes the beam accelerate. In order to keep the protons circulating at the same trajectory, the magnetic and electric field in the 


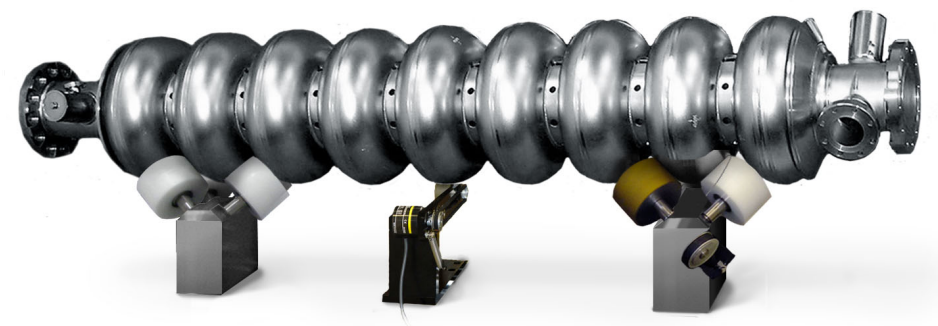

Figure 3.5. Superconducting RF resonator designed at Fermilab.

synchrotron are adjusted synchronously as the beam gets accelerated. The energy to which a particle can be accelerated in a synchrotron is limited by magnet strength. The proton beam in the Booster is accelerated to an energy of $8 \mathrm{GeV}$.

The second synchrotron in the chain is the Main Injector [48]. It is responsible for two different types of activity: antiproton production, and proton-antiproton acceleration before injecting them into the Tevatron. The Main Injector accelerates both antiproton and proton beams from an energy of $8 \mathrm{GeV}$ to $120 \mathrm{GeV}$. As all synchrotrons, it employs a synchronized electromagnetic field for beam acceleration. The antiproton production and acceleration are described in detail in the following subsection.

\subsubsection{Antiproton Creation and Acceleration}

The antiprotons are created by colliding protons with a nickel target. The protons from the Main Injector are divided into 82 bunches and strike a nickel disk $10 \mathrm{~cm}$ in diameter. This produces antiprotons at the rate of 15 antiprotons per 1,000,000 collisions. Besides antiprotons, the incoming $120 \mathrm{GeV}$ protons also produce a shower of other particles that leave the target after the collision. The antiprotons emerge with a 
wide range of angles and velocities. Subsequent focusing is required to achieve a narrow beam with approximately uniform particle kinematics.

The Debuncher [49] is tasked with narrowing down the large momentum spread of the antiprotons. A special lithium lens is used to transfer antiprotons emitted from the target into the Debuncher. The lens collects highly divergent antiprotons, and matches them to a beam with the velocity spread of about $3 \%$. The beam line is subsequently injected into the Debuncher.

The Debuncher is constructed in the form of a rounded triangle with perimeter of 505 meters. The process of reducing the antiprotons momentum spread and forming a compact beam is often referred to as antiproton cooling. In the Debuncher antiproton cooling is done by bunch rotation followed by adiabatic debunching [50]. Adiabatic debunching is a sophisticated cooling technique that allows to reduce the momentum spread to $0.2 \%$. The process of reducing the velocity spread in the Debuncher is often referred to as debunching.

Once the antiprotons are debunched, the beam is transferred to the Accumulator [51] ring where it is also further cooled. The Accumulator is 471 meters in circumference. To produce a high-intensity beam, it employs a special type of cooling, called stochastic cooling [52]. The idea of stochastic cooling is to reduce velocity spread between the particles in the beam using electric signals from an individual particle. Employing this technique for antiprotons allows to obtain higher beam luminosity. The stochastic cooling technique has a simple geometrical visualization in the momentum coordinate system. Each point represents a particle with a specific momentum and therefore a beam of particles can be represented by a three-dimensional figure enclosing such points. In terms of the geometrical representation, the stochastic cooling essentially corresponds to the figure's volume compression in this momentum space. 
The Accumulator repeats the shape of the Debuncher but has a slightly smaller circumference. The injection into the Accumulator occurs every few seconds and recently generated antiprotons are added to a stack of particles already stored in the ring. Over the period of several hours the antiprotons are cooled. As the number of antiprotons in the stack rises the stacking efficiency drops. To maintain high stacking efficiency, the antiprotons have to be transferred to the Recycler. The stochastic cooling technique applied in the Accumulator provides a factor of $10^{6}$ beam phase space compression and makes it possible to utilize the antiproton beam.

The Recycler [53] is an antiproton storage ring 3319 meters long and is located in the same tunnel as the Main Injector. It utilizes permanent magnets to store $8 \mathrm{GeV}$ antiprotons from the Accumulator. The Recycler can retain antiprotons for several hundred hours while keeping beam intensity up to $600 \times 10^{10}$ antiprotons. Additionally, the Recycler is used to receive unused antiprotons from the Tevatron, re-cool and store them until the next injection back into the Tevatron occurs. The cooling in the Recycler ring is done with the aid of an intense electron beam, called electron cooling [54]. The electrons injected into the antiproton beam propagate with the same average velocity as antiprotons. The damping of antiproton momentum oscillations is based on momenta exchange through Coulomb scattering between antiprotons and electrons. When the antiproton momenta are stabilized, the electron beam is separated from the antiprotons. The invention of electron cooling technique made it possible to collide the beams at a very high luminosity. Electron cooling was the main element of Run II Luminosity Upgrade Program of the Tevatron. The antiproton source at Fermilab is able to accumulate nearly $300 \times 10^{9}$ antiprotons per hour.

\subsubsection{The Tevatron}


The Tevatron [56] is proton-antiproton $(p \bar{p})$ collider. Both beams are injected from the Main Injector and share the same tunnel in the Tevatron. The protons are circulating clockwise, and antiprotons counterclockwise. Since the antiproton has the same mass but the opposite charge, it follows the same trajectory in the magnetic and electric field as the proton but in the opposite direction. So the Tevatron equally well accelerates protons and antiprotons in the opposite directions colliding the beams at certain points along the ring.

The approach of setting up two beams in one accelerator ring has tremendous advantage over other types of colliding experiments. First, it eliminates the necessity of having two independent rings as, for example, in the ISR experiment [55]. And second, in contrast to fixed target experiments, there is no energy loss as all the kinetic energy of colliding particles is released in the collision.

The Tevatron is the last synchrotron in the chain. It is made of an accelerator ring $2 \mathrm{~km}$ in diameter and accelerates proton and antiproton bunches injected from the Main Injector to an energy of $980 \mathrm{GeV}$. The Tevatron consists of niobium-titanium superconducting dipole magnets. The magnets are cooled in liquid helium and produce a magnetic field of 4.2 teslas. A large magnetic field is needed to bend the trajectory of the particles and keep them within the pipe. A total of 36 bunches of protons and 36 bunches of antiprotons circulate in the ring. Each proton bunch has approximately $3 \times 10^{11}$ protons, and each antiproton bunch has $3 \times 10^{10}$ antiprotons. Focusing quadrupole magnets installed at the interaction points bring the proton and antiproton beams into head-on collision. The collisions between proton and antiproton bunches occur every $396 \mathrm{~ns}$.

The Tevatron operation is split into a few stages. From 1992 to 1996, the collider was operating at the center-of-mass energy of $1.8 \mathrm{TeV}$ and this period is called Run I. During Run I, the discovery of the top quark occurred. After a series of improvements 
in the next 5 years, the Tevatron started the Run II period in 2001 with a collision energy $1.96 \mathrm{TeV}$. The Run II period, as well as Tevatron running as a whole, was ended on September 30, 2011. Run II consists of two subperiods: Run IIa (2001 - 2006) and Run IIb (2006 - 2011). The main goals of the Run II period were direct searches for unknown particles, including the Higgs boson, and precision measurements such as the quark-mixing matrix elements, properties of the $\mathrm{W}$ and $\mathrm{Z}$ bosons, properties of the top quark and the strong interaction.

\subsection{The DØ Detector}

The $\mathrm{D} \varnothing$ detector [57] is a multipurpose particle detector built to study $p \bar{p}$ collisions produced in the Tevatron. It is designed to identify and measure the four-momentum of final state particles (charged leptons, photons) and hadronic jets. It consists of layers of different types of subdetectors and can be split into three major subsystems: central tracking detectors, uranium/liquid-argon calorimeters, and a muon spectrometer. The following subsections describe the different detector subsystems and give an overview of the trigger and data acquisition (DAQ) system at the end. A detailed description of all subsystems can be found in [58]. Figure 3.6 shows schematic arrangement of the main subsystems in the $\mathrm{D} \varnothing$ detector.

\subsubsection{DØ Coordinate System}

The DØ experiment uses a right-handed Cartesian coordinate system - $(x, y, z)$. The coordinate system is defined with the origin at the center of the D $\varnothing$ detector. The $z$-axis is parallel to the beam pipe and points along the direction of the proton beam. The $x$-axis

points to the center of the Tevatron ring and the $y$-axis is oriented vertically. In addition to the Cartesian coordinate system, cylindrical $(\rho, \phi, z)$ and spherical coordinates $(r, \theta, \phi)$ systems are also widely used. The $\theta$ and $\phi$ angles are the polar and azimuthal angles, 


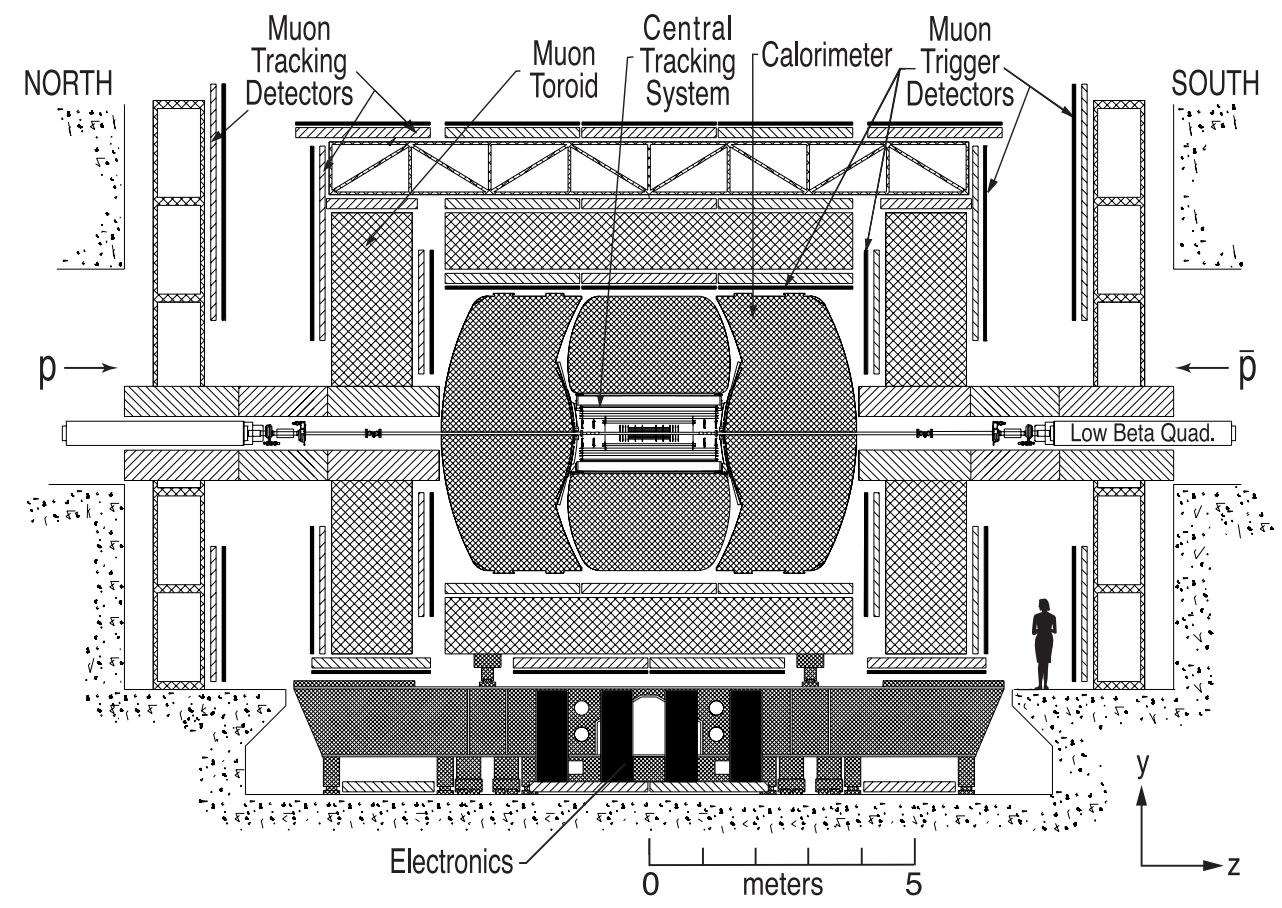

Figure 3.6. Schematic view of the $\mathrm{D} \varnothing$ detector.

respectively, with $\theta=0$ along the beam pipe.

In practice, however, it is more convenient to use pseudorapidity instead of polar angle $\theta$. The pseudorapidity is defined as follows

$$
\eta=-\ln \left(\tan \frac{\theta}{2}\right)
$$

For massless particle or in the relativistic limit, when the energy of a particle is much greater than its mass $(E>>m)$, pseudorapidity is a good approximation of the rapidity of a particle, which is defined as

$$
y=\frac{1}{2} \ln \left(\frac{E+p_{z}}{E-p_{z}}\right)
$$


The rapidity is useful because particles produced via strong interactions tend to be distributed in rapidity approximately uniformly. The rapidity $y$ is an invariant quantity under Lorentz transformation; for example under a Lorentz boost in $z$-direction. It all makes pseudorapidity a preferable choice versus $\theta$.

Proton-antiproton collisions do not necessarily occur in the center of the detector. In fact, the collision vertex can be shifted along the z-axis by as much as $30 \mathrm{~cm}$ due to the longitudinal extension of bunches. The coordinate system with the origin at the interaction point is often referred as physics system, the pseudorapidity and azimuthal angle are denoted with subscript "phys" $\left(\eta_{\text {phys }}, \phi_{\text {phys }}\right)$. The coordinate system with the origin at the center of the detector is referred as detector system with $\left(\eta_{\text {det }}, \phi_{\text {det }}\right)$ correspondingly. The subscripts are often omitted. The physics coordinate system is used when referring to particle kinematic parameters. The detector coordinate system is meant when position relative to the detector is needed, for example, for event selectors.

\subsubsection{Central Tracking System}

The central tracker system consists of a silicon microstrip tracker (SMT) and central fiber tracker (CFT) placed in solenoidal magnetic field of $2 \mathrm{~T}$. It is designed to determine particle momentum, charge and vertex origin, and it also provides useful information for particle identification. The schematic view of the central tracking system is shown in Figure 3.7.

\subsubsection{Silicon Microstrip Tracker}

The SMT provides high precision particle track and vertex reconstruction. The tracker is made of silicon microstrip detectors that contain segments of wafer strips. Essentially, a wafer strip represents a junction of "p" and " $\mathrm{n}$ " type semiconductor material. When a charged particle crosses a silicon wafer, an electron and hole pair is produced. 


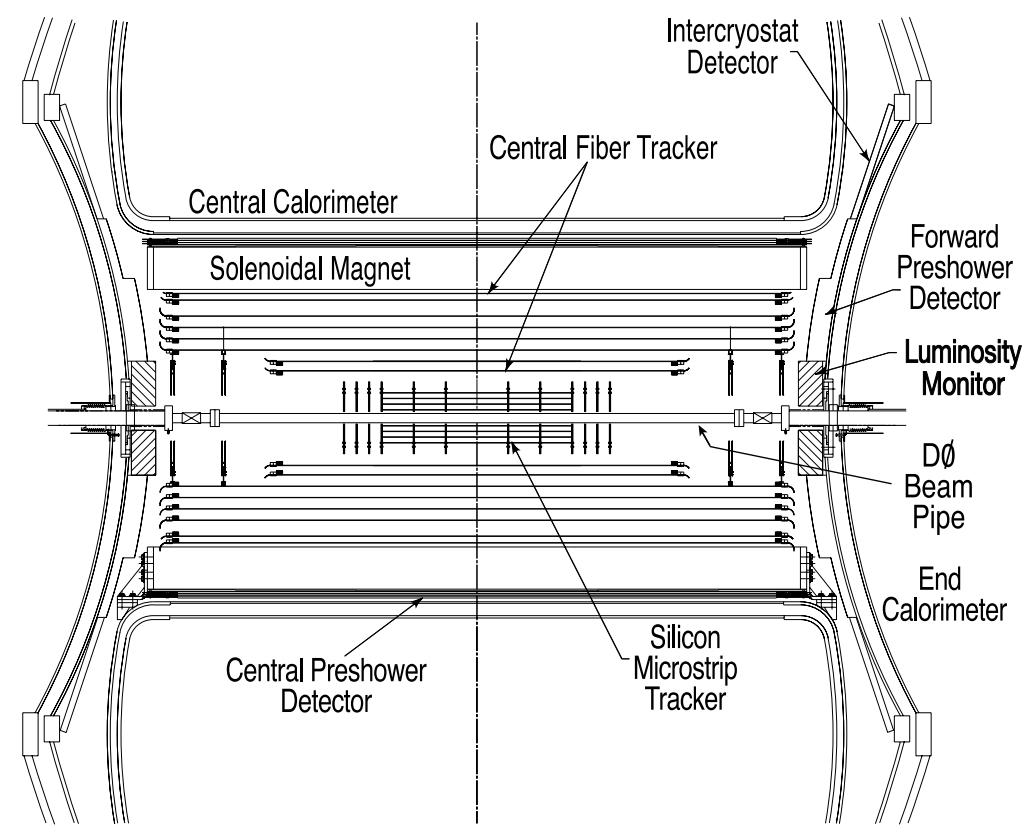

Figure 3.7. Schematic view of the DØ central tracking system.

The coordinates of the point of interaction can be determined by reading out charge generated in the strip. Subsequent measurements across several detectors provides a set of coordinates that allow full reconstruction of the particle's path. The SMT covers a region of tracks with $\eta$ up to 3 .

The tracker design geometry is primarily dictated by the accelerator environment. Large interaction region imposes certain difficulty on the SMT arrangement: the detector surfaces need to be approximately perpendicular to the particle tracks in a wide $\eta$ region, at the same time minimizing dead areas. A dead area is a region that contains no detector elements needed for measurement of particle kinematic parameters or particle detection. For instance, it includes mechanical gaps, support structures, read-out and power cables etc. To accomplish this, the SMT is comprised of barrel and disk modules. There are 
six concentric barrels and twelve F-disks in the central region and four H-disks in the forward region. The barrel detectors are designed to measure the $r-\phi$ coordinate, the disk detectors measure $r-\phi$ and $r-z$. Each barrel has four silicon layers in it. Layers 1 and 2 contain 12 silicon modules each, layers 3 and 4 have 24 modules each. The F-disks are made from 12 wedges in $\phi$, each wedge consisting of double-sided microstrip detector. The H-disks are made of 24 pairs of single-sided microstrip detector. The SMT provides coverage in $|\eta| \leq 3$. The schematic view of the tracker is shown in Figure 3.9.

Silicon can be damaged by possible high temperatures in the SMT. High operating temperature may cause semiconductor type inversion and increase in depletion voltage. Thus efficient cooling system is a crucial component to achieve good performance of the silicon tracker. The SMT cooling system maintains operating temperature below $5^{\circ} \mathrm{C}$ to insure proper detector operation.

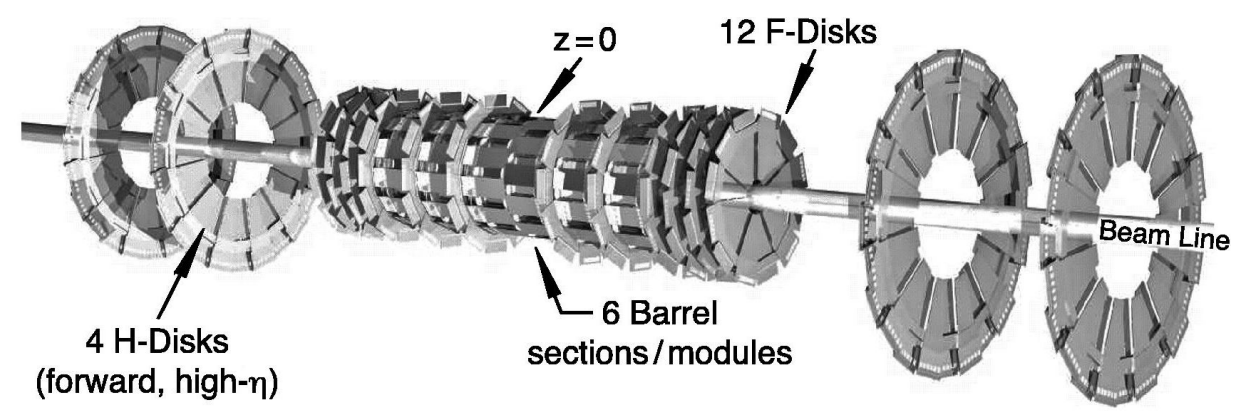

Figure 3.8. Schematic view of the $\mathrm{D} \varnothing$ silicon microstrip tracker.

\subsubsection{Central Fiber Tracker}

The CFT is comprised of multiple layers of scintillating fiber mounted on eight concentric support cylinders. It covers the region $|\eta| \leq 2$. The fibers are made of polystyrene which is doped with an organic fluorescent dye paraterphenyl (PTP). When a charged 
particle passes through a fiber, scintillation light is emitted. A portion of the light travels to the end of the fiber and then transfers to a clear optical fiber waveguide. Through the waveguide the light reaches a Visible Light Photon Counter (VLPC) that converts light into electric signal. The VLPC is a semiconductor device possesing a very high quantum efficiency $(\geq 75 \%)$. It is also very low noise, being operated at liquid He temperature, which allows detection of just a few photons as well as the ability to count photons. The VLPC operation strongly depends on temperature; an increase in temperature by $1 \mathrm{~K}$ gives an increase in the noise by factor of four. To reduce the noise level, the VLPC is loaded into a liquid helium cryostat at the temperature of $9^{\circ} \mathrm{K}$.

The cylinder radius varies from $20 \mathrm{~cm}$ to $52 \mathrm{~cm}$. The two innermost cylinders are $1.66 \mathrm{~m}$ long and the outer six cylinders are $2.52 \mathrm{~m}$ long. The CFT surrounds the SMT and provides good momentum resolution for charged particles. Each cylinder supports two double-layers of fibers termed doublets. The inner doublet has fibers oriented along the beam direction and measures axial coordinate. The outer doublet is placed at the angle of $3^{\circ}$ in $\phi$ relative to the axial layer and provides a stereo measurement. Such configuration permits a measurement of $z$ coordinate of the CFT hit. The doublets are shown in Figure 3.9. Every doublet is made of two adjacent layers of parallel fibers. The fibers are $835 \mu \mathrm{m}$ in diameter and provide position resolution of about $100 \mu \mathrm{m}$.

\subsubsection{Solenoidal Magnet}

To optimize the momentum resolution and tracking reconstruction both SMT and CFT are placed into a homogeneous magnetic field. The superconducting solenoidal magnet [59] surrounds the DØ central tracking system and is $2.73 \mathrm{~m}$ in length and $1.42 \mathrm{~m}$ in diameter. It shares a common vacuum vessel with the $\mathrm{D} \varnothing$ calorimeter. The magnet conductor is made from $\mathrm{Cu}: \mathrm{NbTi}$ wire and produces a magnetic field of $2.0 \mathrm{~T}$ at operating current of 4749 A. The overall thickness of the solenoid is approximately one 


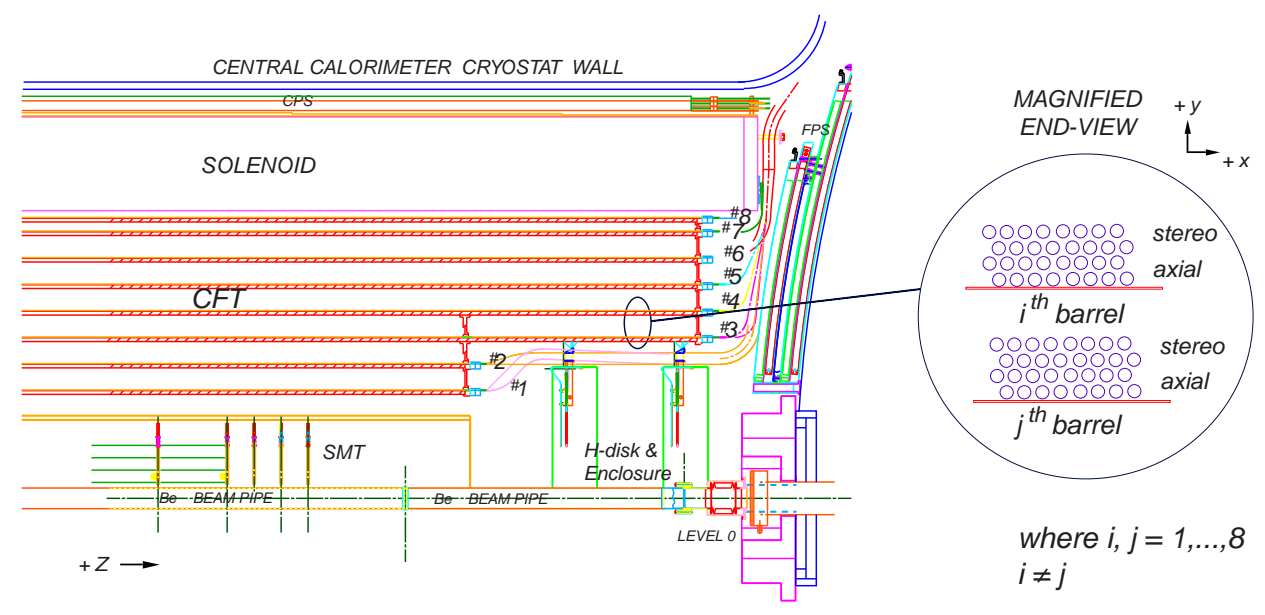

Figure 3.9. Schematic view of the D $\varnothing$ Central Fiber Tracker.

radiation length at $\eta=0$. The choice of thickness is made to optimize the performance of the central preshower detector described in Section 3.2.3.2. The magnet is cooled with liquid helium to the temperature near $4.7^{\circ} \mathrm{K}$. The magnetic field with both the toroid and solenoid magnets turned on is shown in Figure 3.10. The toroid field used for the Muon System is detailed in Section 3.2.4.3.

\subsubsection{Calorimetry}

The calorimeters are designed to measure energies of electrons, photons and jets. They are also important for particle identification and indirect detection of neutrinos via measurement of event transverse momentum imbalance $\left(\not_{T}\right)$. The following subsections describe the Uranium/Liquid-Argon Calorimeter, Preshower Detectors and Inter Cryostat Detector.

\subsubsection{Uranium/Liquid-Argon Calorimeter}




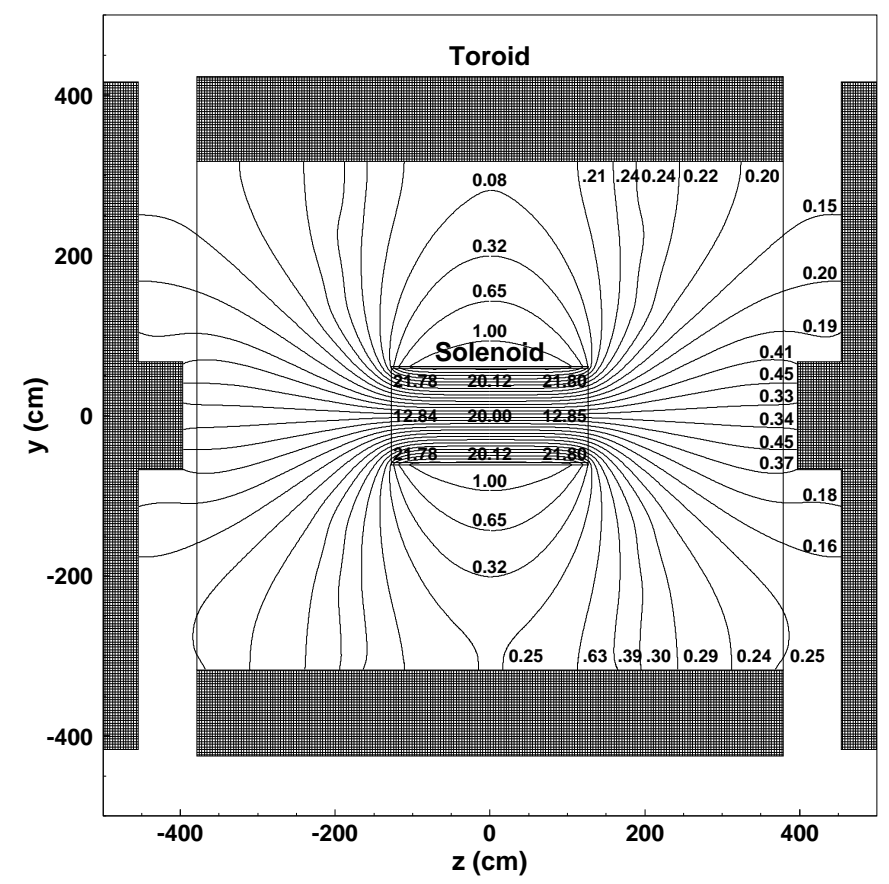

Figure 3.10. A view of magnetic field in the detector (in $\mathrm{kG}$ ). Both the toroidal and solenoidal magnets are on.

The Uranium/Liquid-Argon Calorimeter (U/LAr) is built around the central tracking system. It consists of three main pieces: the Central Calorimeter (CC) and two end calorimeters (EC) - End Calorimeter North (ECN) and End Calorimeter South (ECS). The CC covers a region with $\eta$ up to 1 , while ECN and ECS extend the coverage up to $\eta \leq 4$. Each calorimeter is placed into a separate cryostat and cooled to the temperature $90^{\circ} \mathrm{K}$. The schematic view of the U/LAr is shown in Figure 3.11.

The U/LAr calorimeter (CC, ECN and ECS) contains an electromagnetic (EM), fine hadronic $(\mathrm{FH})$ and coarse hadronic $(\mathrm{CH})$ sections. The EM section is the closest to the interaction region and measures the energies of photons and electrons. The $\mathrm{FH}$ and $\mathrm{CH}$ measure the energies of hadronic jets. Each section is comprised of layers segmented into 
cells. The size of the cells in $\eta \times \phi$ space is $0.1 \times 0.1$ in CC and most of EC $(\eta<3.2)$ for all layers, except for the third EM layer where the size is $0.05 \times 0.05$. There are about 50,000 read-out cells in the U/LAr.

The U/LAr calorimeter is a sampling calorimeter. Typical cell of a sampling calorimeter consists of repeating absorber plates and signal boards. The space between those is filled with an active medium. The absorber plate is a dense material that induces a particle shower in the limited space. The signal board measures shower energy through the ionization of the active medium. The deposited energy is inferred from the amount of liquid argon being ionized. A typical calorimeter cell of the U/LAr is shown in Figure 3.12 .

The active medium of the U/LAr calorimeter is always liquid argon. The absorber material, however, is distinct for different sections. The EM plates employ depleted uranium with width 3 and $4 \mathrm{~mm}$. In the $\mathrm{FH}$ section, a uranium-niobium (2\%) alloy is used with a thickness of $6 \mathrm{~mm}$. The $\mathrm{CH}$ section contains thick $46.5 \mathrm{~mm}$ copper or stainless steel plates. An electric field with a potential of $2 \mathrm{kV}$ is established between the absorber plate and signal board. The gap between the absorber plate and signal board is of $2.3 \mathrm{~mm}$. The time needed for ionization electrons to reach the signal board is less or about $450 \mathrm{~ns}$.

Electromagnetic Shower The first four layers in CC and EC are electromagnetic layers. An electron moving through the EM layer radiate bremsstrahlung photon while the photon produces an electron-positron pair. These two processes are connected: once electron enters absorber it radiates a photon, this photon converts into an electronpositron pair, both electron and positron radiate a photon and the process repeats. Thus a single high-energy electron produces a shower of lower energy particles that travel in the same direction as the electron. Showering stops when the energy of an electron falls below 


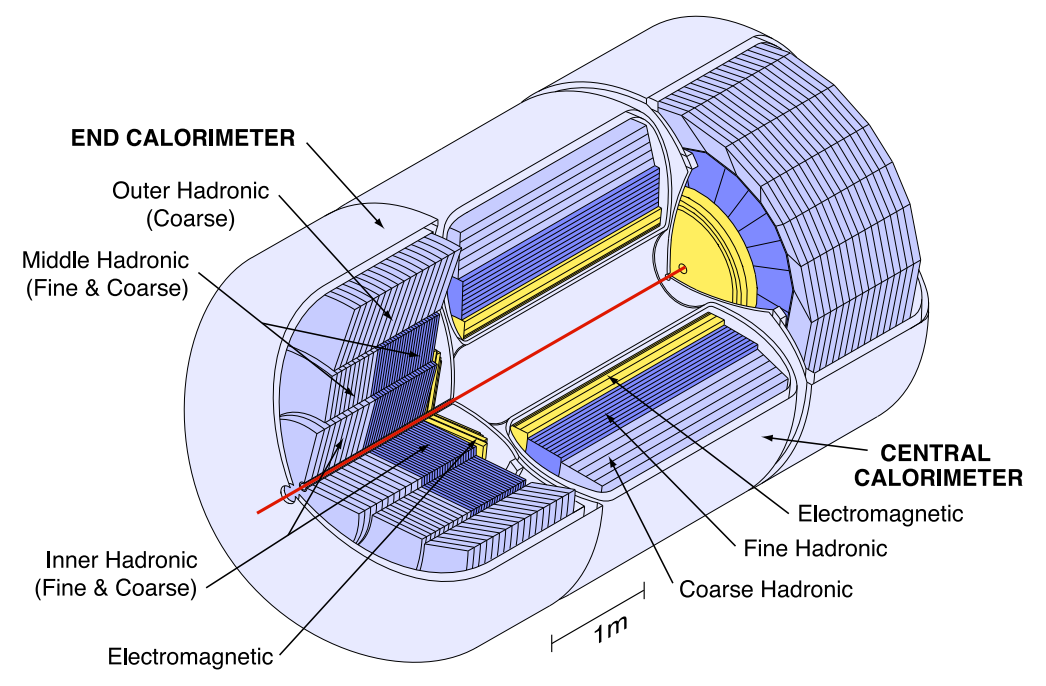

Figure 3.11. Schematic view of the DØ Liquid Argon Calorimeter.

some critical value $E_{\text {brem }}$. At the energies below $E_{b r e m}$, the main energy loss process is due to ionization. A similar process occurs if the incident particle is a photon. The EM captures most of the energy from electrons or photons. A typical electromagnetic shower is depicted in Figure 3.14. Radiation losses through bremsstrahlung by highenergy electrons can be described in terms of $X_{0}$,

$$
\frac{d E}{d x}=-\frac{E}{X_{0}}
$$

where $E$ is the energy of the electron and $X_{0}$ is radiation length. The radiation length is an average distance over which a high-energy electron loses $\left(1-e^{-1}\right)$ of its energy by bremsstrahlung. The length for a high-energy photon to convert into electron-positron pair equals $\frac{9}{7} X_{0}$ and is termed a conversion length. Typical scales of radiation length are $X_{0}=0.32$ for uranium, $X_{0}=1.43$ for copper. The EM section of the CC region of the $\mathrm{U} / \mathrm{LAr}$ has a thickness of 21 radiation lengths. 


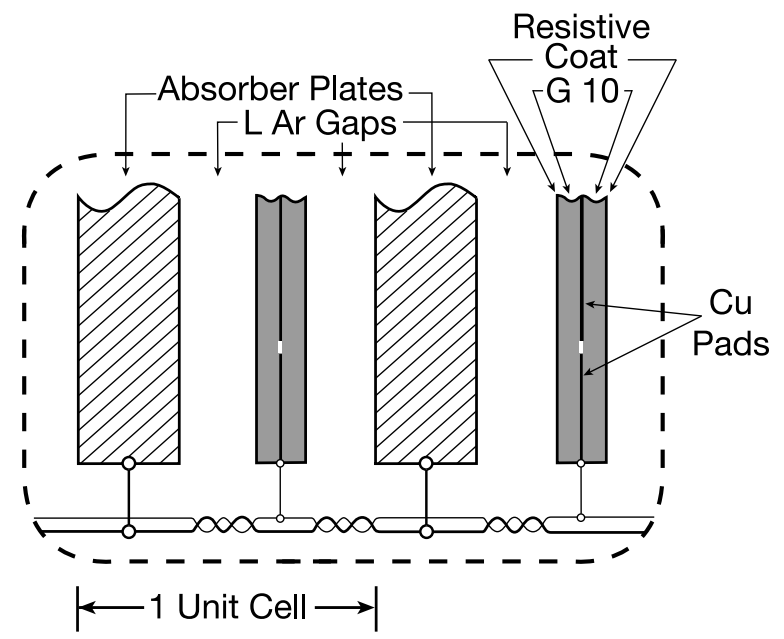

Figure 3.12. Schematic view of the liquid argon cell for the calorimeter.

Hadronic Shower The next four layers in the CC after EM are hadronic layers. The first three layers are fine and the fourth layer is coarse. The EC is divided into three regions: inner, middle and outer. Each region is made of the similar layers as the EM, except that absorber material and plate thickness are different. The schematic view of the portion of the U/LAr showing layers arrangement in $\mathrm{CC}$ and $\mathrm{EC}$ is shown in Figure 3.13. Entering the layer, a hadron from the jet interacts with absorber material via the strong interaction. New hadrons produced at the interaction, mostly pions and nucleons, further interact inelastically with the nuclei. The process repeats producing a hadronic shower. These interactions ultimately produce electrons and positrons that cause ionization of the liquid argon.

A schematic view of a hadronic shower in calorimeter is shown in Figure 3.15. Similarly to $X_{0}$ in EM, hadron shower development can be described in terms of average nuclear interaction length $\lambda_{I}$. The interaction length depends on the type of absorber material used and grows with atomic number. For calorimeters $\lambda_{I}$ is much larger than 


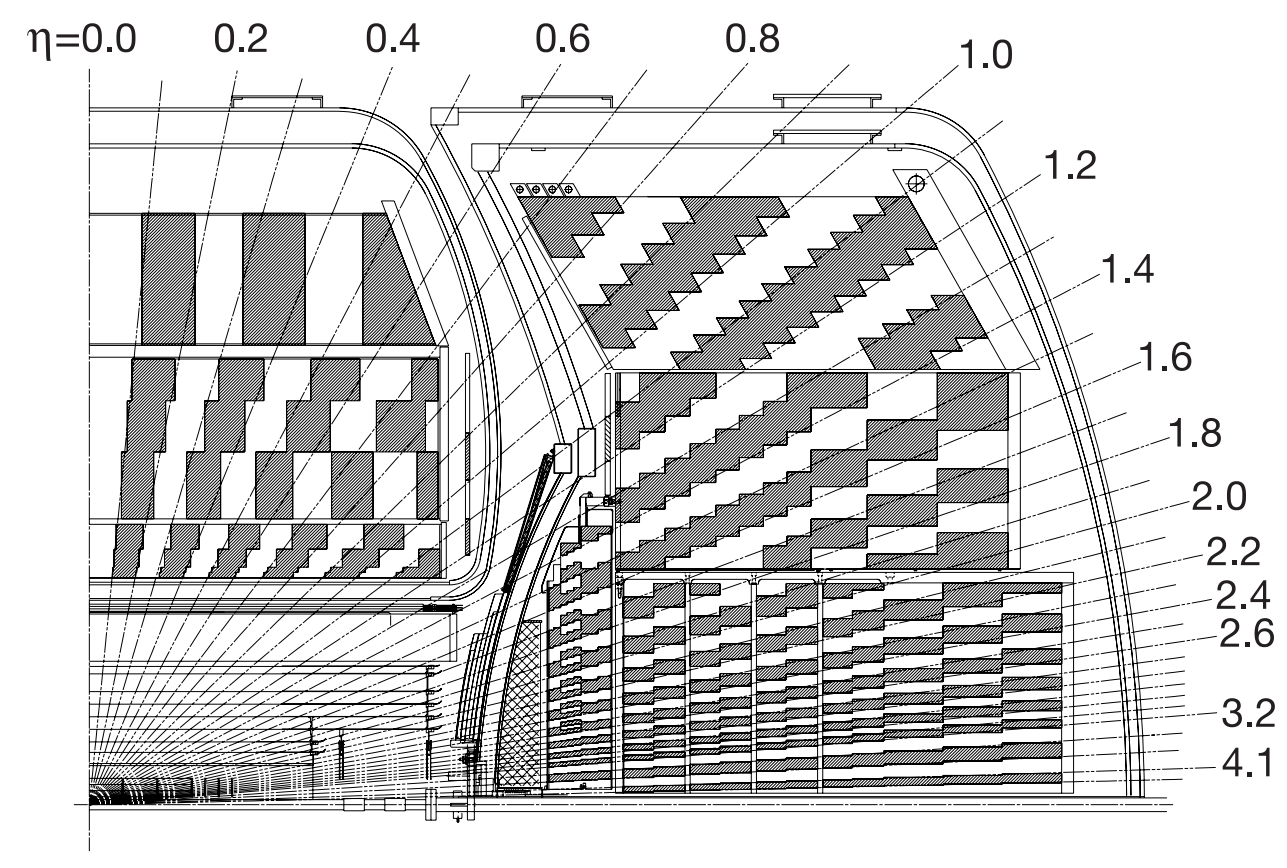

Figure 3.13. Schematic view of the portion of the U/LAr showing layers and tower arrangement.

$X_{0}$. This fact justifies thicker absorber in $\mathrm{FH}$ and $\mathrm{CH}$ as compared to EM. The total thickness of the U/LAr varies from 7 to 9 interaction lengths.

\subsubsection{Preshower Detectors}

The preshower detectors are designed for particle identification and background rejection during online and offline reconstruction. They combine features of calorimeter and tracking detector. As a calorimeter, the preshower samples the incident particle energy and as a tracker, it provides precise position measurement. The Central Preshower (CPS) is placed in the gap between the solenoid and the calorimeter. It covers the 


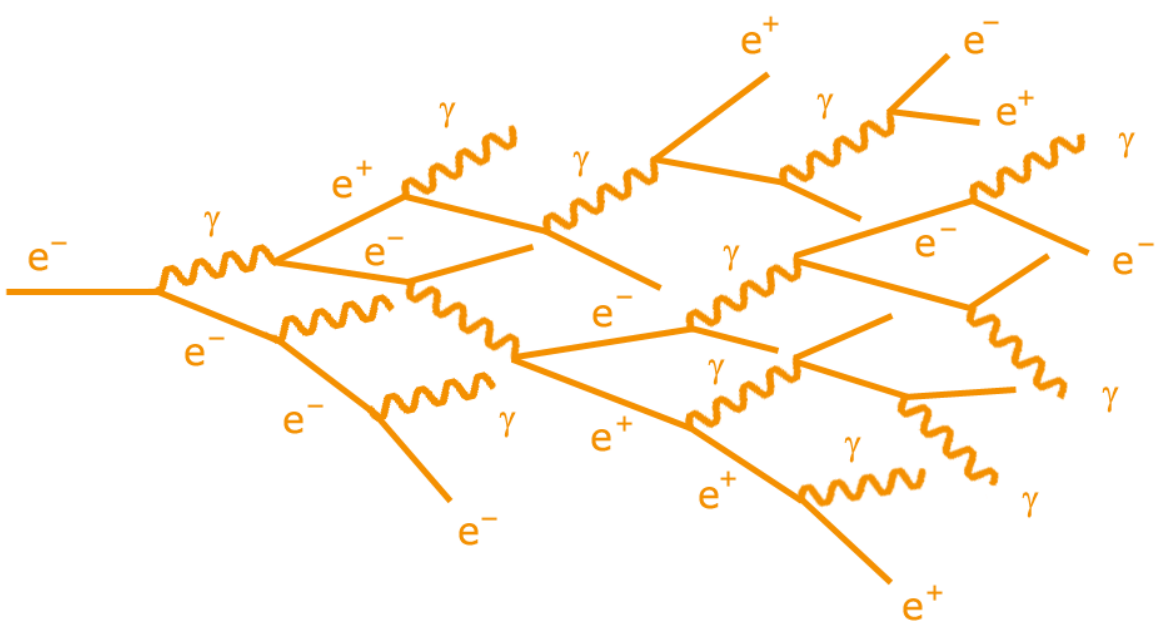

Figure 3.14. Schematic view of an electromagnetic shower.

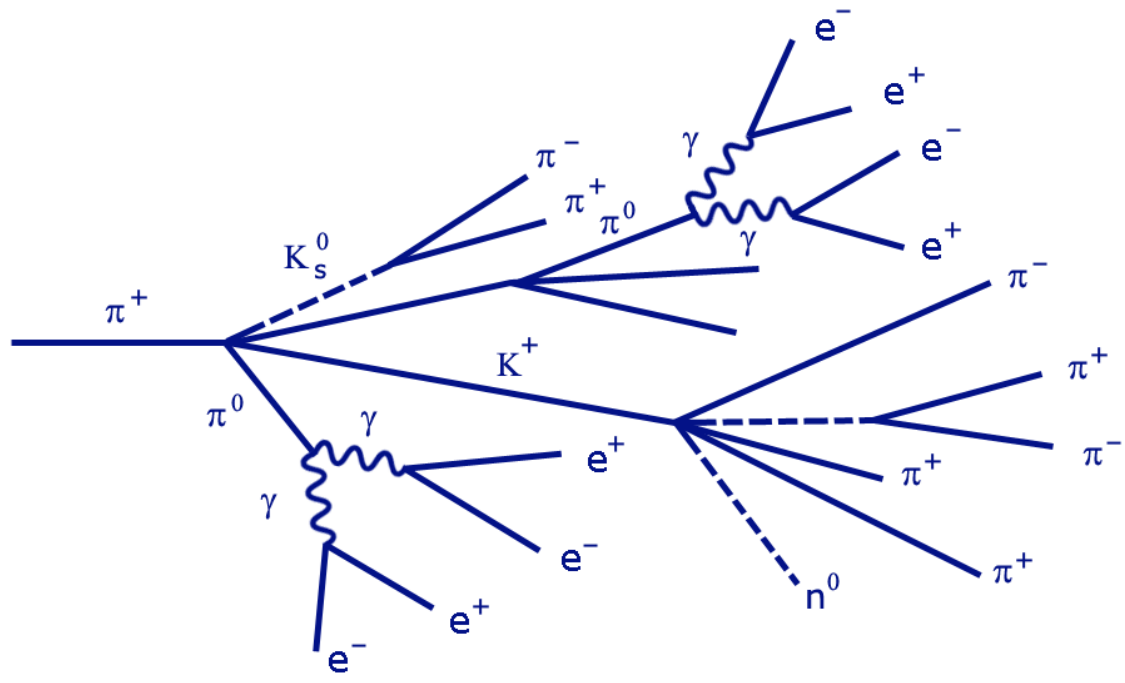

Figure 3.15. Schematic development of hadronic shower in calorimeter. 
pseudorapidity region $|\eta|<1.3$. The Forward Preshower (FPS) is mounted on the front wall of the EC cryostat. The FPD provides coverage in $1.5<|\eta|<2.5$. The preshower detectors are shown in Figure 3.7.

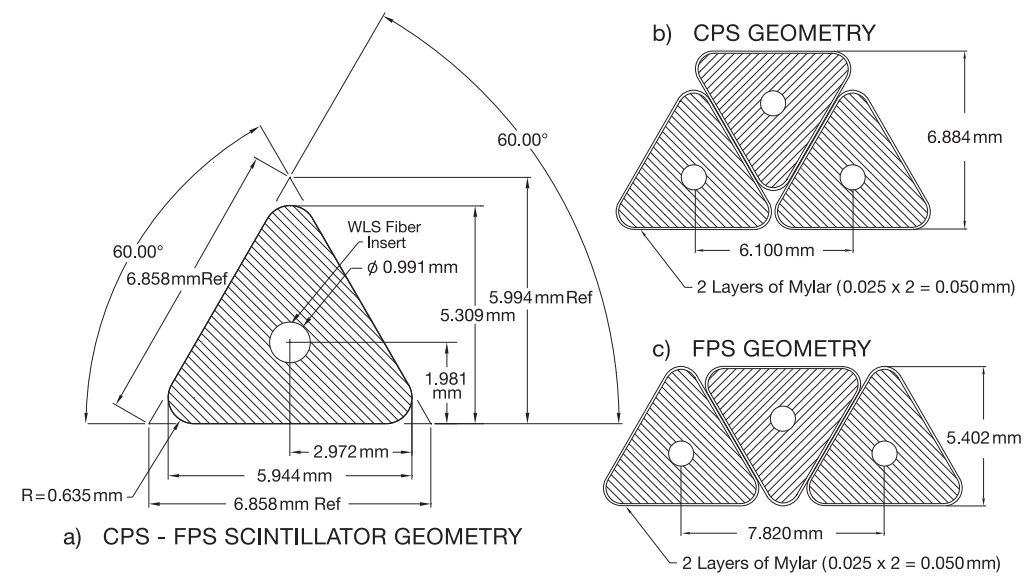

Figure 3.16. Schematic view of cross section of the CPS and FPS scintillator strips.

The CPS is made of lead absorber with two radiation lengths followed by three layers of triangular scintillator strips. Passing through the solenoid and absorber, an electron or photon produces an EM shower. The shower energy is measured by the scintillator layers of the CPS. Since the interaction length for hadrons is much greater than the radiation length for electrons and photons, the probability of hadronic interactions in the CPS is very low. Thus the preshower detector can aid in distinguishing hadronic particles from electrons and photons.

The scintillator layers consist of one axial and two stereo layers of the strips. The two stereo layers are placed on the top of the axial at stereo angles of $24^{\circ}$ and $-24^{\circ}$. At the center of each triangular strip, as shown in Figure 3.16, there is embedded fiber. When a particle passes through the strip, the fiber collects and carries the scintillation 
light from the strips to the end of the detector. Subsequently the light is read out by VLPC attached at the end of the fiber.

The (FPD) is made of $22.5^{\circ}$ wedges mounted on the faces of the EC cryostats. Every edge consists of two scintillator layers in the front, $11 \mathrm{~mm}$ lead-stainless-steel absorber with a radiation length of $2 X_{0}$ in the middle, and two scintillator layers in the back. The innermost layer is used to detect particles based on ionization they produce. It is also called the minimum ionization particle (MIP) layer. The outermost layer is for detecting EM shower and therefore it is called shower layer. Passing through a wedge, a charged particle will leave some ionization in the MIP layer allowing position measurement of the track. Electrons and photons produce EM shower in the absorber that results into cluster of energy in the shower layer. Hadronic particles just pass through leaving no shower in the absorber. The charged hadrons, however, do leave a MIP trace read out by VLPCs.

The preshower detectors and the central fiber tracker rely on common elements. The waveguides and the readout electronics system are shared between CFT and the preshower detectors.

\subsubsection{Inter Cryostat Detector}

The Inter Cryostat Detector (ICD) is a scintillator sampling layer attached to the exterior surfaces of the end cryostats. It covers the region $1.1<|\eta|<1.4$, as can be seen in Figure 3.13. The need of the ICD is dictated by the incomplete calorimeter coverage and substantial presence of unsampled material in that region.

The ICD is made of 0.5 "-thick scintillating tiles enclosed in aluminium boxes. Each tile covers a region $\Delta \eta \times \Delta \phi \approx 0.3 \times 0.4$ and is divided in 12 subtiles. Each subtile covers $\Delta \eta \times \Delta \phi \approx 0.1 \times 0.1$. The ICD is shown in Figure 3.17.

The ICD electronics is placed outside of the $\mathrm{D} \emptyset$ detector in a low-magnetic field and 
designed to be compatible with the calorimeter read-out system. The signal from tiles to the electronics is transferred through wavelength shifting fibers. There are a total of 378 channels in the ICD.

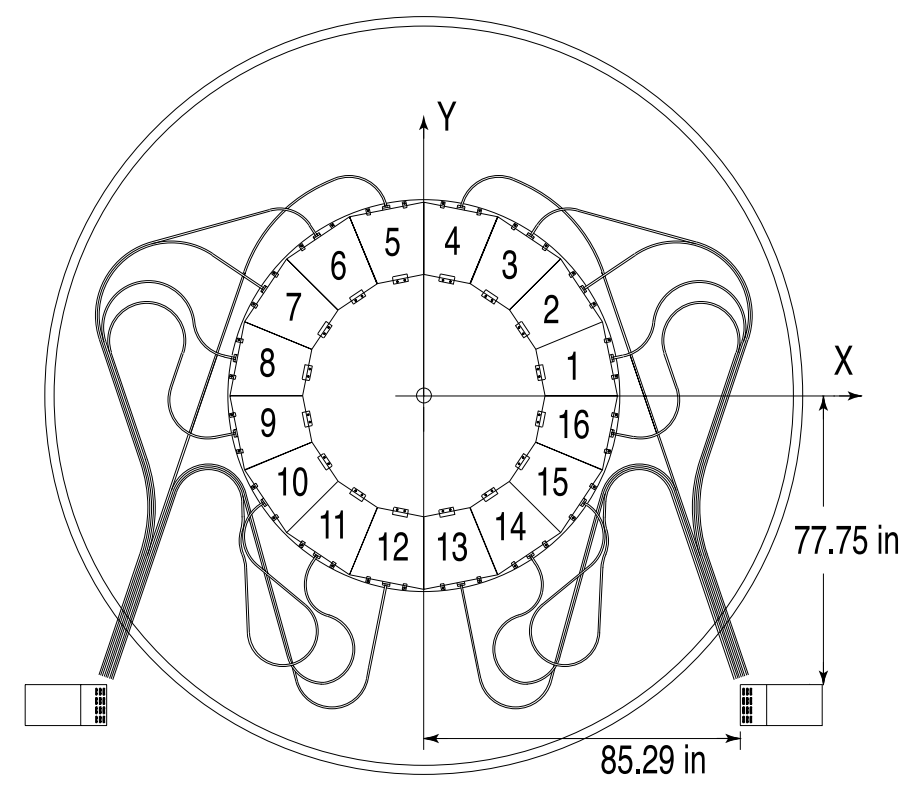

Figure 3.17. Cross section view of the ICD tiles on the endcap cryostats. The rectangles represent an assembly where ICD electronics is installed.

The ICD is used to improve jet resolution by removing non-gaussian tails. It is also important for obtaining good $E_{T}$ resolution when jets are in the region $1.1<|\eta|<1.4$.

\subsubsection{Muon System}

The Muon System is the outermost subdetector and it is used to identify muons and to complement the CFT in the muon momentum measurement. In addition to identifying muons, the Muon System is used to discriminate against cosmic muons (muons that are coming from the atmosphere). Because of the muon's large mass, the bremsstrahlung 
radiation is not a dominating energy loss process. The main energy loss occurs through the ionization. Thus the muons are the only particles that can reach the Muon System whereas electrons, photons and hadrons shower and stop in the calorimeter.

The Muon System is comprised of drift tubes, scintillators and a toroid magnet system. The scintillators are used for triggering, the drift tubes are employed for precise track position measurements and triggering as well. The Muon System is divided in two regions by coverage in $\eta$ : the central region that covers $|\eta|<1$ and the forward region $1<|\eta|<2$. Each region of the system is made of layers of drift tubes and scintillators. The closest to the calorimeter is layer "A," then follows layer "B" and "C", respectively. A toroid magnet is located between layers A and B.

\subsubsection{Drift Tubes}

Proportional Drift Tubes (PDTs) are employed in the central region. They are made of rectangular extruded aluminum and collected together in the drift chambers. Typical size of the drift chamber is $2.8 \times 5.6 \mathrm{~m}^{2}$. Each PDT layer in the detector is made up of decks of individual cells. There are four decks in A-layer except of the bottom of the detector which have three decks. The B and $\mathrm{C}$ layers consist of three decks only. The PDT layers are shown in Figure 3.18.

Each single PDT cell is a rectangular-shaped aluminium enclosure with a size of $10.1 \times 5.5 \mathrm{~cm}^{2}$. There are anode wires at the center and two cathode pads above and below the anode wire in every cell. A high voltage of $2300 \mathrm{~V}$ is applied for the pads and $4700 \mathrm{~V}$ for the wires. The cells are filled with circulating non-flammable gas mixture. The mixture is $\mathrm{Ar}(84 \%), \mathrm{CH}_{4}(8 \%)$ and $\mathrm{CF}_{4}(8 \%)$. The gas recirculates with the flow rate of 200 liters per minute. During the circulation the gas is being filtered to remove contaminants.

A muon passing through the tube will ionize the gas and create an avalanche of 


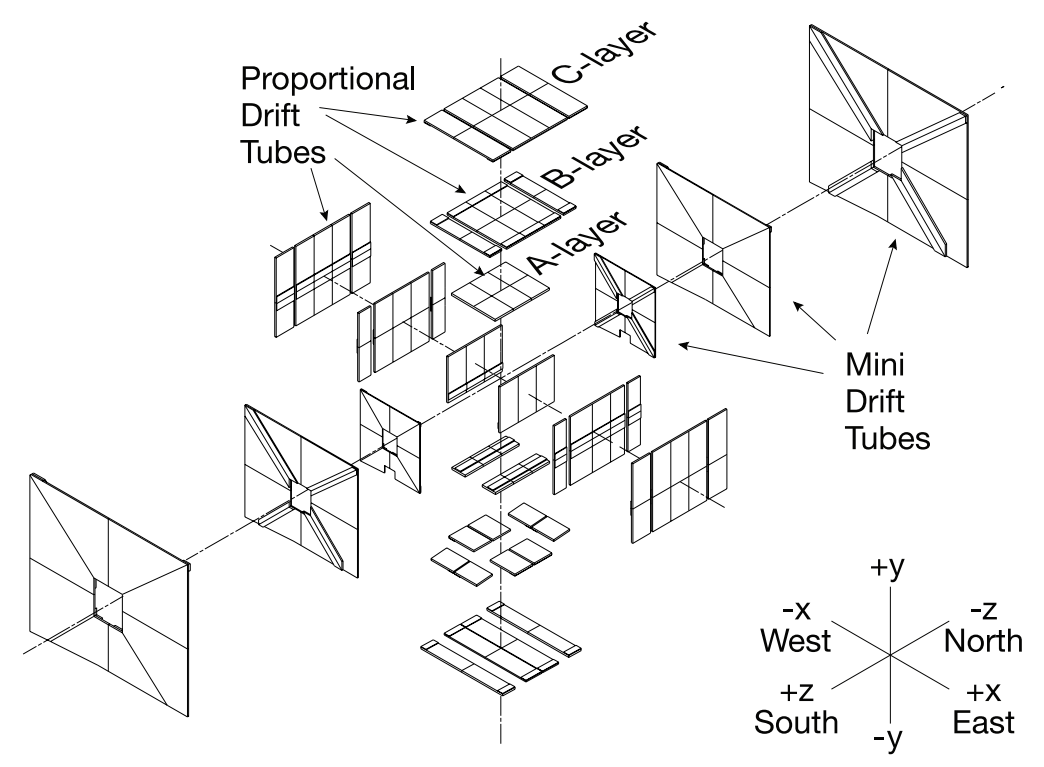

Figure 3.18. Schematic view of the muon wire chambers.

electrons under the high voltage in a certain cell. The avalanche of electrons will drift to the anode for the time $\Delta T$. The drift time $\Delta T$ and the charge collected at the anode are used to determine the hit position along the wire. The PDTs are positioned perpendicularly to the beam pipe and provide single-cell coordinate resolution of about $1 \mathrm{~mm}$.

Moving in the toroid magnetic field, a muon trajectory is getting bent. The curvature of bend of the track is used to determine the muon momentum. However, the momentum for a reconstructed muon is provided by the CFT due to its better momentum resolution. The PDT is used to find the matching track of a muon.

The maximum drift time for the PDT is $450 \mathrm{~ns}$. Since this is higher than the time between collisions, a muon track in the PDT must be also confirmed in the scintillator counter. The PDT system consists of 6624 cells. 
The drift tubes in the forward muon system are called Mini Drift Tubes (MDTs). The MDTs are positioned perpendicular to the beam pipe and have four decks of cells in the A-layer, and three decks in each B and C-layer. An MDT consists of eight cells each of size $9.4 \times 9.4 \mathrm{~mm}^{2}$. Each mini drift tube cell is made of a comb-shaped thin-wall aluminium profile with 50- $\mu m$ gold-plated tungsten anode wire in the center. Anode wires are supported by plastic spacers installed at one meter interval. The cathode is made of the aluminium profile and 0.15 -mm-thick stainless steel cover placed on the top of it. To provide the electrical insulation and mechanical stability, the structure is enclosed in a 1-mm-thick plastic envelope. Figure 3.19 shows cross-sectional view of the MDT. The MDT system is filled up with $\mathrm{CF}_{4}-\mathrm{CH}_{4}$ gas mixture: $90 \% \mathrm{CF}_{4}$ and $10 \%$ $\mathrm{CH}_{4}$. This mixture shows no radiation aging and has a wide operational range. The operating high voltage is $-3200 \mathrm{~V}$ for the cathode, and the anode wire is grounded at the amplifier. The MDT operates similarly to PDT but with significantly shorter timing the maximum drift time for the MDT is $60 \mathrm{~ns}$. The entire MDT system consists of 6080 tubes and contains 48,640 cells.

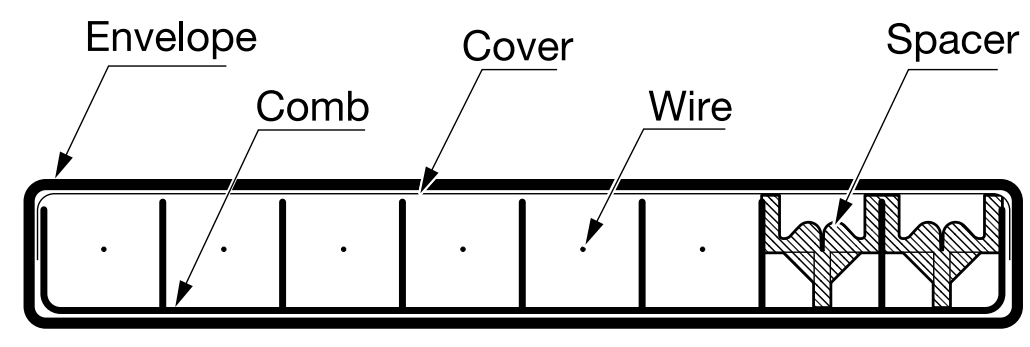

Figure 3.19. Schematic view of a mini drift tube.

The MDT momentum resolution is limited by multiple scattering in the toroid and the coordinate resolution of the CFT. For muons with momenta below $40 \mathrm{GeV}$, the standalone MDT momentum resolution is about 20\%. The CFT determines an overall 
muon momentum resolution for muons with energies below $100 \mathrm{GeV}$; the MDT is used for higher momentum muons and muons in the forward region $1.6<|\eta|<2.0$ where the CFT does not have layers. The MDT hit position resolution is about $0.7 \mathrm{~mm}$.

\subsubsection{Scintillators}

To provide precise timing information for muon triggering and muon trajectory reconstruction from muon chambers, the Muon System is equipped with layers of scintillators. Good timing resolution allows to trigger on muons from proton-antiproton collision and rejecting cosmic muons. There are two layers of scintillators in the central region: "A $\phi$ " scintillators are inside of the central toroid, "cosmic cap" are located outside of the magnet. There are also three layers of trigger scintillation counters in the forward region. The layers of the scintillators are schematically shown in Figure 3.20.

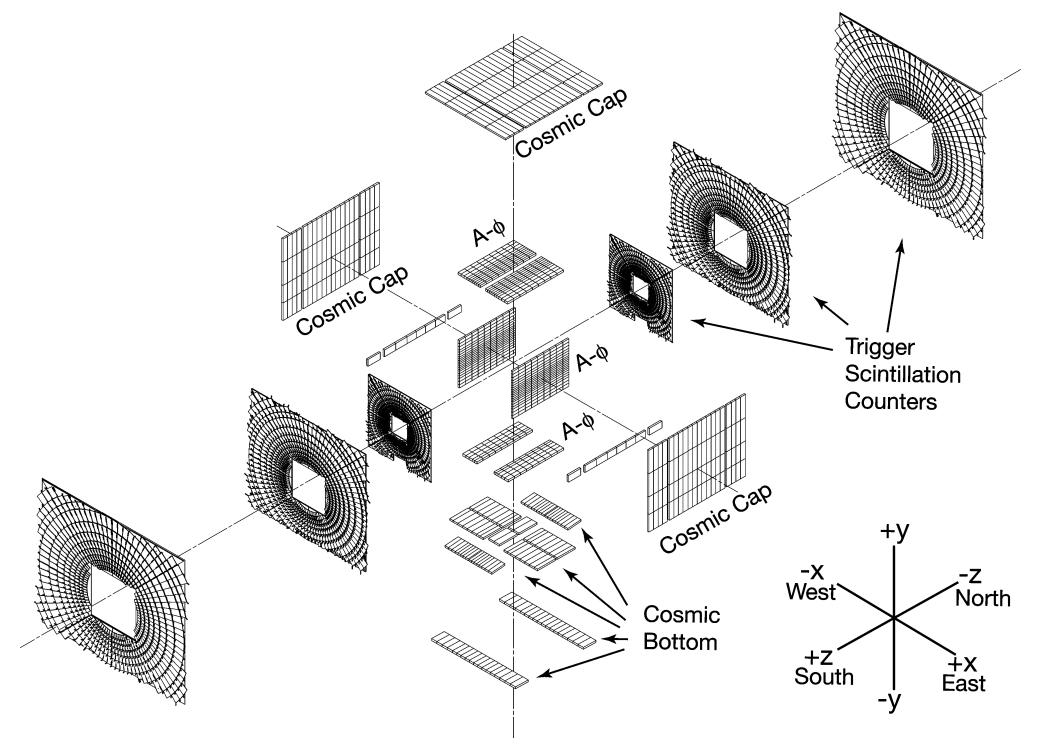

Figure 3.20. Schematic view of the muon scintillation detectors.

The cosmic cap [60] are placed on the top, sides and bottom of the PDTs in the C- 
layer. They are made from grooved 0.5" Bicron 404A scintillator [61]. The Bicron 404A scintillator is chosen because of its low cost and good performance with no significant degradation due to radiation damage. The scintillation light is collected by four $1 \mathrm{~mm}$ diameter Bicron BCF91A wavelength shifting (WLS) fibers [62] that are placed in each groove. The fibers are gathered at the center of the counters and read out by 1.5" 10 stage EMI [63] 9902KA phototubes. The counters are oriented with their widths along $z$ and lengths along $\phi$-direction except bottom cap that positioned with their narrow dimension along $\phi$ and their long dimension along $\eta$. The counters is approximately $4.5^{\circ}$ in $\phi$ and their length is equal to respective PDT's.

The A $\phi$ scintillator counters are in the A-layer. They are made of 12.7 -mm-thick Bicron 404A scintillator in a manner similar to the bottom cosmic cap. The counters segmentation is chosen to be $4.5^{\circ}$ in $\phi$ to match the CFT trigger segmentation. The counters have timing resolution of about 2 ns and their primary goal is to provide precise timing information to match a track in the CFT with a hit from $\mathrm{A} \phi$. This is particularly important to register low- $p_{T}$ muons that can not traverse the toroid. The counters operate in a residual magnetic field of 200-350 G from the toroidal and solenoidal magnets. There are $630 \mathrm{~A} \phi$ counters in the Muon System.

The muon trigger scintillation counters are installed in all three layers A, B and C. The counters design is similar to the cosmic cap and A $\phi$ counters. It uses 0.5" Bicron 404A scintillator cut to a trapezoidal shape with two 4.2mm-thick and 0.5"-wide WLS bars. The bars are installed on the sides of the counter and collect light onto a phototube. To reduce the magnetic field, the phototubes are placed in metal shields.

In each layer, the trigger scintillation counters are arranged in $r-\phi$ geometry and divided into octants containing about 96 counters. As in the case of the cosmic cap, $\phi$-segmentation is $4.5^{\circ}$ and made to match the CFT trigger sectors. The largest layer is the C-layer with size of approximately $12 \times 10 \mathrm{~m}^{2}$. The C-layers is shown in Figure 


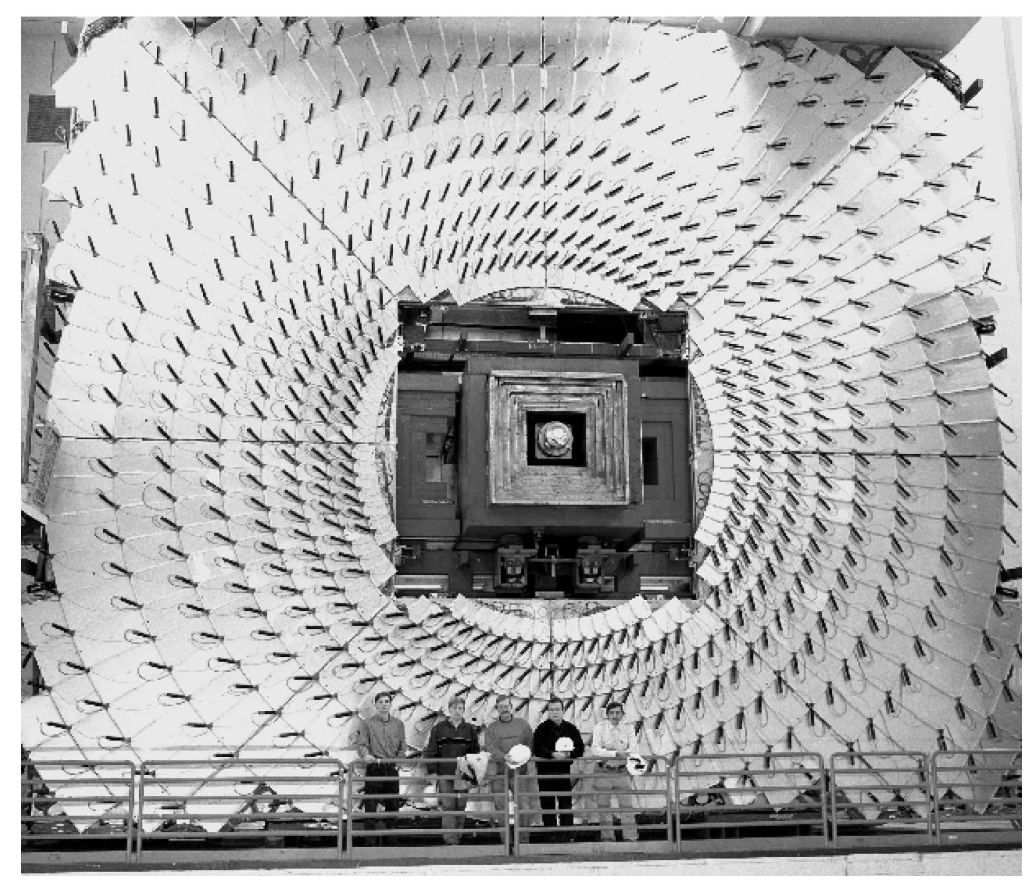

Figure 3.21. The picture of the trigger scintillation counters in the C-layer.

3.21. During the test beam study, the counters showed time resolution of about 1 ns and detection efficiency of $99.9 \%$.

\subsubsection{Toroidal Magnet}

The Muon System contains toroid magnet that is arranged in three pieces: central toroid and two end toroids. Similarly to the CFT, the magnetic field is used to optimize the momentum resolution and tracking reconstruction of muons. The toroid magnet is decribed in details in Ref. [57]. The central toroid is $109 \mathrm{~cm}$-thick square annulus of iron placed about $318 \mathrm{~cm}$ from the Tevatron beampipe. The two end toroids are made in C-shapes and located $447 \mathrm{~cm}$ from the center of the detector.

The field in the central and end toroids is approximately $1.8 \mathrm{~T}$ and $1.9 \mathrm{~T}$ respectively. 
All three pieces of the toroid magnet are linked in series and operate at a current of 1500 A. The magnetic field with both the toroid and solenoid magnets turned on is shown in Figure 3.10. The toroid field used for the Muon System is detailed in section 3.2.4.3.

\subsubsection{Triggering and Data Acquisition System}

With $2.5 \mathrm{MHz}$ bunch crossing in the Tevatron, there are more than 2 million collisions occuring every second. However, not all events produced in the collisions contain interesting physics processes. In fact, only a small fraction of events are needed to be selected and recorded for physics analyses. Therefore, the D $\varnothing$ experiment uses sophisticated trigger and data acquisition systems to filter the events. The main goal of a trigger system is to select "interesting" events and reject background events at maximum efficiency.

Most of the collisions result in multijet events. Decaying heavy particles such as $W$ and $Z$ bosons, and top quarks may produce high $p_{T}$ leptons, very energetic hadronic jets, and large momentum imbalance that can be used as distinctive signatures of interesting physics. The D $\varnothing$ trigger system consists of three distinct layers and exploits such signatures for determining interesting events.

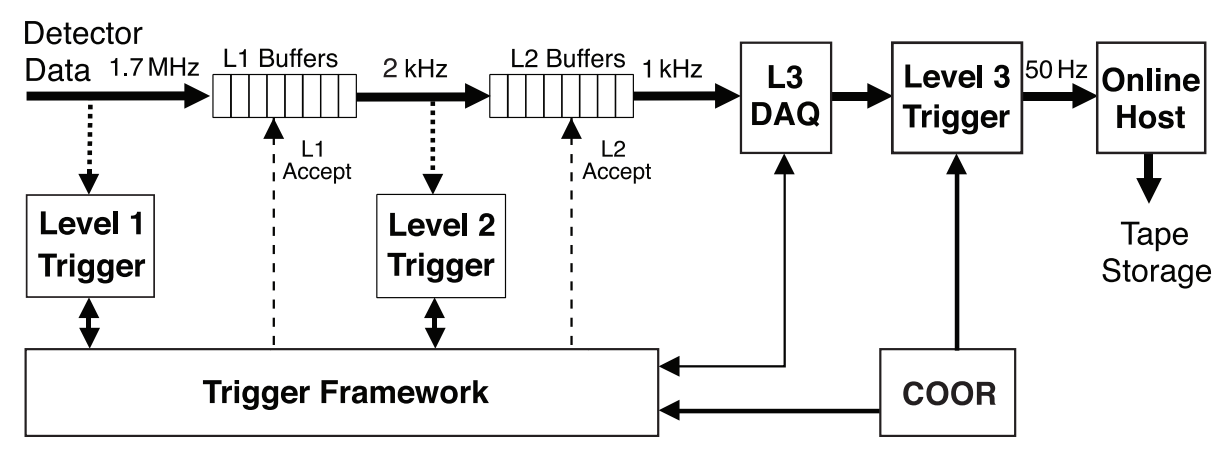

Figure 3.22. Overview of the $\mathrm{D} \varnothing$ trigger and data acquisition systems. 
The first stage, or Level 1 (L1), triggers is a hardware-based system with an accept rate of about $2 \mathrm{kHz}$. It provides initial selection based on the information from a subset of detectors. The Level 2 (L2) trigger is constructed of number of preprocessors and a global processor. At L2, the trigger decision is based on individual reconstructed objects and their spatial correlation. The L2 system has an accept rate of $1 \mathrm{kHz}$ and can handle input rates of up to $10 \mathrm{kHz}$. Passing through L1 and L2, the event is sent to Level 3 triggers (L3). The L3 system is a farm of computers that employs sophisticated software algorithms to make the final decision. The L3 algorithms are similar to those used in the offline analyses, and the accept rate is $50 \mathrm{~Hz}$. The data acquisition system is responsible for transferring fully digitized events to the L3 system. An overview of the D $\varnothing$ trigger and data acquisition system can be seen in Figure 3.22.

\subsubsection{Level 1 Triggers}

The Level 1 system consists of the calorimeter trigger (L1Cal), the central track trigger (L1CTT), the muon system trigger (L1Muon) and the forward proton detector trigger (L1FPD). The Trigger Framework gathers the information from each L1 trigger and make a decision whether to keep or reject the event. The Level 1 system is capable of making a decision in less than $3.5 \mu \mathrm{s}$.

The L1Cal looks for transverse energy deposits above preset thresholds. It is designed to trigger on events with high- $p_{T}$ electrons, photons, jets, taus decaying into hadrons, as well as events with large imbalance of momentum.

The L1CTT performs fast track reconstruction from scintillators in the CFT and the preshower detectors. Besides the trigger decision itself, the L1CTT electronics also keeps additional data of the event for further processing at L2/L3 triggers. The L1CTT is used separately or together with L1Muon trigger.

The L1Muon seeks patterns consistent with muon tracks originating from the center 
of the detector. It uses hits from muon wire chambers, muon scintillation counters and tracks from the L1CTT. Subsequently the track transverse momentum is compared with preset thresholds to make a trigger decision on the event. Most of the comic muons do not have tracks originated from the center of the detector and therefore do not pass the trigger. The other cosmic muons are rejected by the trigger based on the time discrepancy between the muon track and the beam crossing.

\subsubsection{Level 2 Triggers}

The L2 trigger system is split into two stages: detector subsystems preprocessing and a global stage (L2Global) that exploits for correlations in physics signatures across all subsystems. Preprocessor subsystems include tracking, calorimeter, preshower and muon systems. At the preprocessing stage, the information is collected from the front ends and L1 triggers. It is then further analyzed to form primitives such as EM clusters, tracks, jet clusters, etc. Combining the data from all subsystems, L2 forms a physics objects: electrons, photons, muons, etc. Based on the objects identified at the preprocessing stage, the L2Global makes a trigger decision. The objects have to satisfy configuration requirements loaded into the L2Global.

The L2 triggers include the following preprocessors: the calorimeter (L2Cal), the muon (L2Muon), the preshower (L2PS), the central tracking (L2CTT), the SMT (L2STT). The L2Cal identifies jets, electrons and photons. It also determines event transverse momentum imbalance $\left(E_{T}\right)$ based on the individual trigger tower energies passed from L1 trigger. The L2Muon uses timing data to find muon track segments and match those segments with L1Muon and fiber tracker trigger information. It is designed to further improve the quality of the muon candidates from L1Muon trigger. The L2PS, L2CTT and L2STT are detailed in Ref. [58]. 


\subsubsection{Level 3 Triggers}

A fully digitized event is sent to the L3 trigger after passing through the L2 trigger. The L3 system is a high-level trigger system that employs programmable software algorithms to make trigger decisions. Such algorithms are usually called "the filters" and run on a computing farm under the Linux operating system. The filters make a decision based on the information of completely reconstructed physics objects (electrons, photons, jets, etc). Making a decision, the filters also account for the relationship between the objects. For example, calculations are performed of the rapidity or azimuthal angle separating physics objects or their invariant mass. The specialized software is utilized by the filters to perform object reconstruction and some other low-level work as unpacking raw data, applying calibration, forming clusters, locating hits etc. Physics objects are precisely defined in the "reference sets" (refsets) that are input to the tools. A refset is essentially a collection of pre-defined algorithm parameters. For instance, jet refsets specify jet cone size, electron refsets set electromagnetic fraction. An event passes the L3 trigger if at least one filter is satisfied. After positive L3 trigger decision, the event is sent to the host cluster for recording.

L3 trigger has an input rate from the L2 system of $1 \mathrm{kHz}$. This provides about 235 ms per event available for processing. The actual time needed to input, build and output one event through the L3 trigger is nearly $15 \mathrm{~ms}$. The output rate for data recording is $50 \mathrm{~Hz}$. The L3 trigger system is designed to operate with instantaneous luminosity up to $1.5 \times 10^{32}$ which was exceeded in Run2 by a factor of 3 . There are currently 252 L3 nodes.

\subsubsection{Data Acquisition System}

The data acquisition system (DAQ) provides event data transfer from L2 read-out crates to L3 farm nodes. The DAQ is designed for data transfers at the maximum rate 
of $250 \mathrm{MB} / \mathrm{s}$. That corresponds to $200 \mathrm{kB}$ of data per event at the L2 accept rate of $1 \mathrm{kHz}$. The $\mathrm{D} \varnothing$ triggering is controlled and operated by the central coordination program COOR. COOR is running on an online host system and receives requests from user to configure the detector or to start and stop runs. To carry out user requests, COOR sends commands directly to L1 and L2 triggers, and the DAQ system.

Passing L3 trigger data is transported to the online host system. L3 filtering farm sends event data with the rate of approximately $50 \mathrm{~Hz} \times 200 \mathrm{kB}=10 \mathrm{MB} / \mathrm{s}$. Online monitoring host performs various data and detector monitoring tasks. Eventually through the online host system, the data is further transferred to the final repository to be recorded on tapes. The tapes repository is located about $3 \mathrm{~km}$ from the D $\varnothing$ detector at Feynman Computing Center. 


\section{Chapter 4}

\section{DATA SAMPLE AND SIMULATION}

This chapter details the data sample analyzed. It also gives an overview of Monte Carlo simulation technique and describes simulated samples that are required for the top quark mass measurement with the method used in this thesis.

\subsection{Data Sample}

This analysis is based on data collected by the $\mathrm{D} \emptyset$ experiment during time periods described in section 4.1.1. The collected data was reconstructed with the DØ reconstruction software D0reco. The vertices, tracks, EM clusters and other objects were identified according to the methods detailed in the Section 5.2.

The full dataset is reduced to smaller subsamples in a process called skimming. Smaller subsamples include only required physics signatures for a particular analysis. Smaller size datasets greatly reduce a time needed for further samples processing and event selections. In case of dilepton final states skimmed data consist of the EMMU sample, the 2EMhighpt and the 2MUhighpt samples. The EMMU sample includes events with one high $p_{T}$ reconstructed EM cluster and one Muon candidate. The 2EMhighpt skim has events with two high $p_{T}$ reconstructed EM cluster, and the 2MUhighpt skim contains events with two high $p_{T}$ Muon candidates. The dataset skimming is done by the DØ Common Samples Group [64] using the software framework.

The skimmed data is stored at the $\mathrm{D} \varnothing$ computing cluster in the form of ROOT based files. ROOT [65], [66] is an object oriented framework that allows us to store and analyze large scale data for high energy physics experiments. Based on the ROOT file format, 
the data files additionally comply with the Common Analysis Format (CAF). The CAF uses ROOT TTree or simply "tree" as its basic storage mechanism. It was developed at $\mathrm{D} \varnothing$ and is used by CAF software framework in various physics analyses. Following CAF standard, the data contains the detector data (calorimeter cells, tracker hits, etc), trigger information, reconstructed objects, and particle identification information (btagging, for example).

\subsubsection{Data Time Periods}

The data used in this analysis was collected by the $\mathrm{D} \varnothing$ experiment between June 2006 and September 2011 of Run II of the Tevatron. This period corresponds to an integrated luminosity of $4.3 \mathrm{fb}^{-1}$. The final result is combined with the result from a similar analysis carried on the Run IIa data, which was taken between April 2002 and February 2006 and corresponds to an integrated luminosity of $1.0 \mathrm{fb}^{-1}$.

\subsubsection{Data Quality}

This analysis uses the data that meet predefined data quality criteria. Those requirements ensure that all subsystems were operating correctly at the time of data taking. Any subsystem malfunction may result in serious data quality issues. For example, inadequate operation of the calorimeter may produce very large signals in certain cells or even entire tower of calorimeter cells. It also might be possible that a signal was not registered at all due to improper calorimeter calibration, e.g. incorrect pedestal substraction. Therefore constant online data quality monitoring is an important part of data taking procedure.

During data taking, the data is being recorded in time periods called luminosity blocks. Every such block lasts about two minutes. The instantaneous luminosity in a block is considered to be constant and equals the average luminosity for that period. 
The total integrated luminosity is calculated by summing up the luminosity blocks that satisfy data quality requirements.

Besides the collision data itself, each block also contains status information of every detector subsystem. If a subsystem does not function properly, we flag it as bad. A block can be declared as bad if any subsystem status during the data taking is marked as bad. The $\mathrm{D} \varnothing$ data quality framework also allows us to check the data at a later stage. Such re-assessment is termed offline monitoring. The information about the status of a luminosity block is stored in a special database and is available for offline data quality reprocessing. The offline reprocessing is performed by the $\mathrm{D} \varnothing$ data quality group and provides additional data quality checks based on the information saved in the database.

For every analysis a list of bad luminosity blocks is created. The list of bad blocks depend on the list of triggers required in the analysis. Events that occurred in bad luminosity blocks are eliminated and only good events are analyzed. Luminosity blocks can be declared as bad, for example, due to bad calibration constants, unexpected large noise in the calorimeter, constant energy calorimeter ring in $\phi$ that reflects problem with electronics etc.

\subsubsection{Trigger Requirements}

To enrich the data sample with events that have two leptons in the final state, certain trigger selections are needed. The triggers used in the analysis are specific to each channel and have different requirements.

In the ee channel, each event is required to pass at least one of the triggers in a list of single electron triggers. All single electron triggers require an EM calorimeter tower to be above a certain $E_{T}$ threshold at Level 1. In addition, the tower has to match with the hits in the tracking system. For the $\mu \mu$ channel, each event is required to fire at least one of a set of single muon triggers. All single muon triggers require a track match at 
Level 1 and are therefore restricted to $|\eta|<1.6$. At the highest luminosity they require track isolation and tight wire conditions.

Efficiencies for single $e e$ and $\mu \mu$ triggers have been measured in the $Z \rightarrow e e$ and $Z \rightarrow \mu \mu$ event sample. The efficiency for an electron to pass a specific trigger requirement is calculated using the tag-and-probe method. One electron in the sample is chosen as a tag electron. The tag electron is required to satisfy one of the Level 1 single electron trigger requirements. The second electron is defined as a probe electron. The probe electron has no trigger requirements and is considered an unbiased object from which to measure an efficiency. The total trigger efficiency is approximately $99 \%$ for the ee channel and $80 \%$ for $\mu \mu$.

For the $e \mu$ channel, to maximize the trigger efficiency, no explicit trigger selections were applied. All available triggers that represent a mixture of single and multilepton triggers and lepton+jet triggers were used. The trigger efficiency for the $e \mu$ channel is nearly $100 \%$. It is evaluated by folding single trigger efficiencies in the MC simulation.

The triggers used in this thesis correspond to v15, v15.50 and v16 versions of trigger lists. Employed trigger lists are detailed in Ref. [67]. Details on any given trigger can be obtain from Trigger Database at the DØ TriggerMeisters' web-page [68].

\subsection{Monte Carlo Simulation}

The top quark mass measurement presented in this thesis is performed in part by comparing SM simulation with the data. To simulate signal and background events, Monte Carlo (MC) event generators were used. A detailed description of the methods used to perform such an analysis is given in Chapter 7.

$\mathrm{MC}$ event generators are an integral part of almost any experimental analysis in high energy physics. They are a very important tool to relate theoretical predictions with the data measurements. Based on the theoretical models of fundamental interactions and 
results from phenomenology, MC generators simulate events using combination of numerical and theoretical methods. Event generators are called Monte Carlo because they exploit special numerical integration methods that are referred as Monte Carlo methods. The Monte Carlo methods are an alternative approach of estimating integrals. Fast and efficient integral estimation is a key aspect of calculations in high energy physics. MC generators provide a proper kinematic modeling of a signal and background processes. With regard to the top quark mass measurement, events simulated with MC generators are used to calibrate the method before we run the measurement on the real data.

High energy collision is comprised of several stages: hard scattering, parton showering, partonic decays, and parton shower evolution (hadronization). A MC event is simulated by one or more event generators depending on the choice of an analyzer as generators are not perfect. Event generators can be general purpose that simulate every stage of a collision (e.g. PYTHIA [69]) or those that perform just part of the process, for example computing hard-scattering process (e.g. ALPGEN [70]). Once simulated by MC generator(s), an event is then processed through a GEANT (GEometry ANd Tracking) [71] based simulation of the D $\varnothing$ detector. GEANT is a software program designed to describe the passage of elementary particles through matter. Passing through the GEANT reconstruction, the MC simulation is then overlayed with minimum bias events. The minimum bias is due to secondary interactions of the other constituent partons of the colliding hadrons. Minimum bias events are collected in data with no physics trigger required, except the Level 0. At Level 0, the minimum bias trigger scintillators select elastic $p \bar{p}$ interactions. Adding minimum bias to the simulation events allows close imitation of the detector data. Subsequently the simulation is passed through the same trigger requirements and reconstructed with the same software as for the detector data.

\subsubsection{Hard scattering}




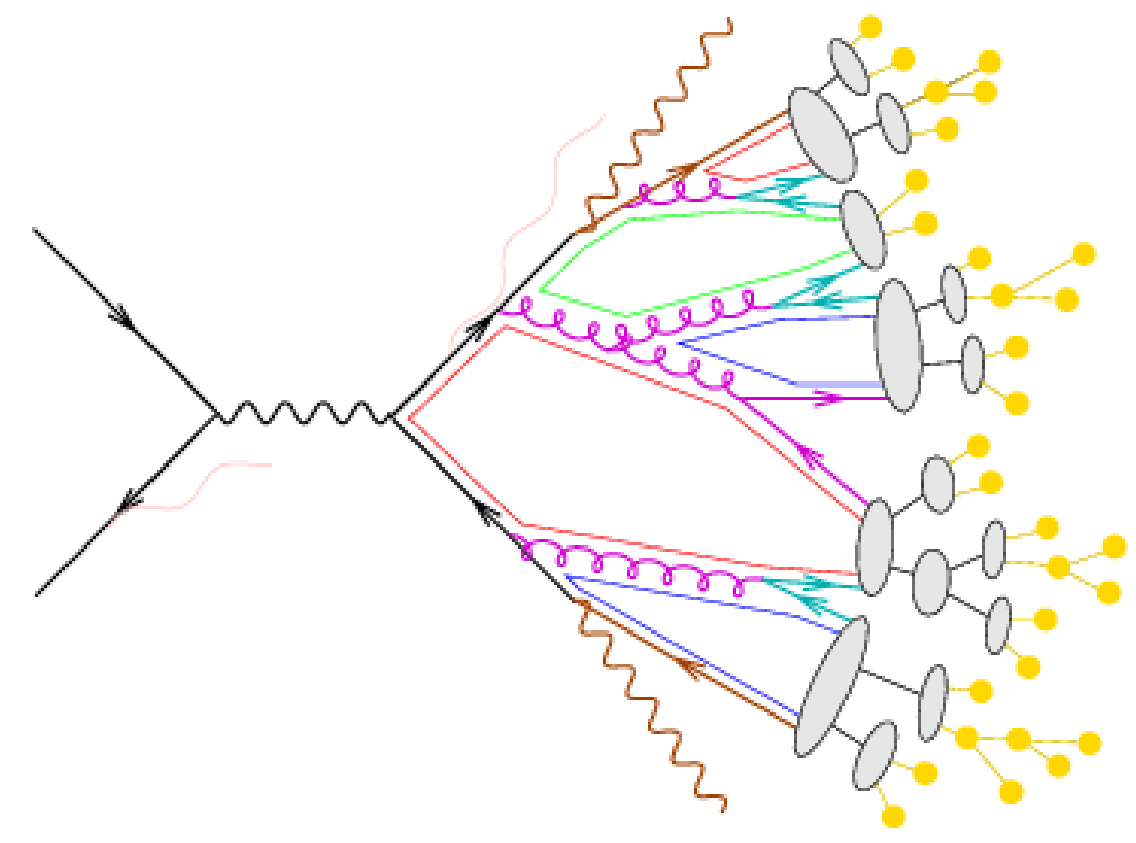

Figure 4.1. Schematic view of hard scattering process with subsequent parton showering and hadronization.

Colliding hadrons can be viewed as a mixture of partons quarks and gluons. At a time when an inelastic collision occurs, two partons interact with each other. That gives the hard process of interest and is associated with large invariant momentum transfer. Most of the collisions, however, are filtered away as they do not include the process of interest. Those interactions are mostly result in low transverse momentum objects and soft jets.

The hard process of interest simulation is the core of any simulation of collider events with use of MC event generators. In order to address the physics of interest a large number of processes are embedded in MC generators. Since partons and gluons are asymptotically free, the high-energy interactions between them can be described by 
perturbation theory. That enables a possibility to compute many features of the interactions using Feynman diagrams. In practice, the hard scattering process is calculated either to leading order (LO) or next-to-leading (NLO) order Feynman diagrams due to various technical and theoretical complications. Those include non-perturbative QCD calculations, the virtual corrections calculations, and others.

One of the central tasks for MC generators is calculation of hard scattering cross section. The cross section of a process defines the likelihood of interaction between particles. Based on the Lagrangian approach in quantum field theory, the Feynman rules can be derived. From the Feynman rules the matrix elements can be calculated. Integrating the matrix element over the particle's phase space the process cross sections can be calculated.

To describe a proton-antiproton interaction, the cross sections have to be convoluted with the flux of the incoming partons $\mathrm{i}$ and $\mathrm{j}$ in the two incoming hadrons $\mathrm{A}$ and $\mathrm{B}$ :

$$
\sigma=\sum_{i, j} \iiint d x_{1} d x_{2} d \hat{t} f_{i}^{A}\left(x_{1}, Q^{2}\right) f_{j}^{B}\left(x_{2}, Q^{2}\right) \frac{d \hat{\sigma_{i j}}}{d \hat{t}}
$$

$f_{i}^{A}\left(x_{1}, Q^{2}\right)$ and $f_{j}^{B}\left(x_{2}, Q^{2}\right)$ are the parton distribution functions (PDF). PDFs is defined as the probability density for finding a particle of a given flavor $(i, j)$ in the proton or antiproton at momentum transfer $Q^{2}$ with a certain longitudinal momentum fraction $x_{1}$ and $x_{2}$ correspondingly. The cross section is specified only for a given PDFs. PDFs are non-perturbative quantities and cannot be computed from first principles. Using different phenomenological models PDFs are extracted from the data. All generators are already pre-set with commonly used PDF sets, e.g. CTEQ, MRST.

$d \hat{\sigma_{i j}} / d \hat{t}$ is differential cross section of a process. It defines the likelihood of registering final state particles at some angle with respect to the colliding beams. The differential cross section is computed from matrix element. For example, the invariant differential 
cross section for $2 \rightarrow 2$ particle scattering:

$$
\frac{d \hat{\sigma}}{d \hat{t}}=\frac{1}{64 \pi \hat{s}\left|\overrightarrow{p_{i}}\right|^{2}}\left|M_{f i}\right|^{2}
$$

where $t$ and $s$ are Mandelstam variables: $\hat{s}=\left(p_{1}+p_{2}\right)$ - squared sum of 4-momentum of the incoming particles, $\hat{t}=\left(p_{1}-p_{3}\right)$ - square of the four-momentum transfer. $\left|\overrightarrow{p_{i}}\right|^{2}$ is

a constant, fixed by energy and momentum conservation $\left|\overrightarrow{p_{i}}\right|^{2}=\frac{1}{4 \hat{s}}\left[\hat{s}-\left(m_{1}+m_{2}\right)^{2}\right][\hat{s}-$ $\left.\left(m_{1}-m_{2}\right)^{2}\right] . M_{f i}$ is matrix element to observer final state $f$ from the initial state $i$. All multi-purpose event generators provide a LO matrix element calculation in the Standard Model and some new physics extensions for $2 \rightarrow 1,2 \rightarrow 2$ channels. Dedicated matrixelement generators, like ALPGEN [70] and MadGraph [73], are capable to simulate higher-multiplicity final states.

\subsubsection{Parton showering}

The particles entering and leaving the hard scattering are typically QCD partons carrying a "color" charge. The parton would emit gluons in the same way that electrically charged particles emit photons. Unlike photons, the gluons can also radiate other gluons and produce quark-antiquark pairs. Thus a single parton can generate a shower of outgoing partons that is also called showering. Emission associated with colliding partons is called Initial State Radiation (ISR). Outgoing parton emission is called Final State Radiation (FSR).

Unlike the hard scattering process that is described usefully by lowest-order matrix elements, parton showering has to include the higher order effects to give a complete picture of the process. The effect of all higher orders is simulated through a parton shower algorithm that is based on some simplifications. The simplifications used in the showering algorithm allow to described the structure of emissions to the low momentum transfer scales of order $1 \mathrm{GeV}$. The parton showering algorithm utilizes the Markov chain 
approach of successive parton emissions. Using the probabilistic notion of emission to occur, one or more partons is added to the final state during each step in the chain. Another different formulation of parton showering is based on a successive emissions from the colored dipoles formed by pairs of partons. Parton shower algorithms are used in the PYTHIA [69] and HERWIG [74] generators.

\subsubsection{Hadronization}

When the scale of momentum transfers is low (of order $1 \mathrm{GeV}$ ), hadron formation occurs as the ending stage of the parton showering. The parton shower evolution cannot currently be described from first principles, as QCD theory is non-perturbative at low energies. Thus, phenomenological models are necessary to describe the process of hadronization.

The main phenomenological models used today are string fragmentation [75] and cluster fragmentation [76]. To describe the hadron-level properties of final states, the string fragmentation model is used in PYTHIA. A central idea of this model is that partons hadronize in a color-connected mode forming color-neutral systems. The model suggests a separate confinement field, or a string, stretched between each color and its matching anticolor. Such a field can break producing new quark-antiquark pairs. A quark from one break can combine with an antiquark from another break to form a final state hadron. A string may also include gluons. They act as kinks carrying energy and momentum, and thus the string is considered as comprised from several pieces. Hadronization along each of the string pieces proceeds in the same way as for a single straight string. The process of forming and breaking strings repeats iteratively until there is not enough of energy for production of a hadron. Many hadrons are unstable and further decay to lighter particles. 

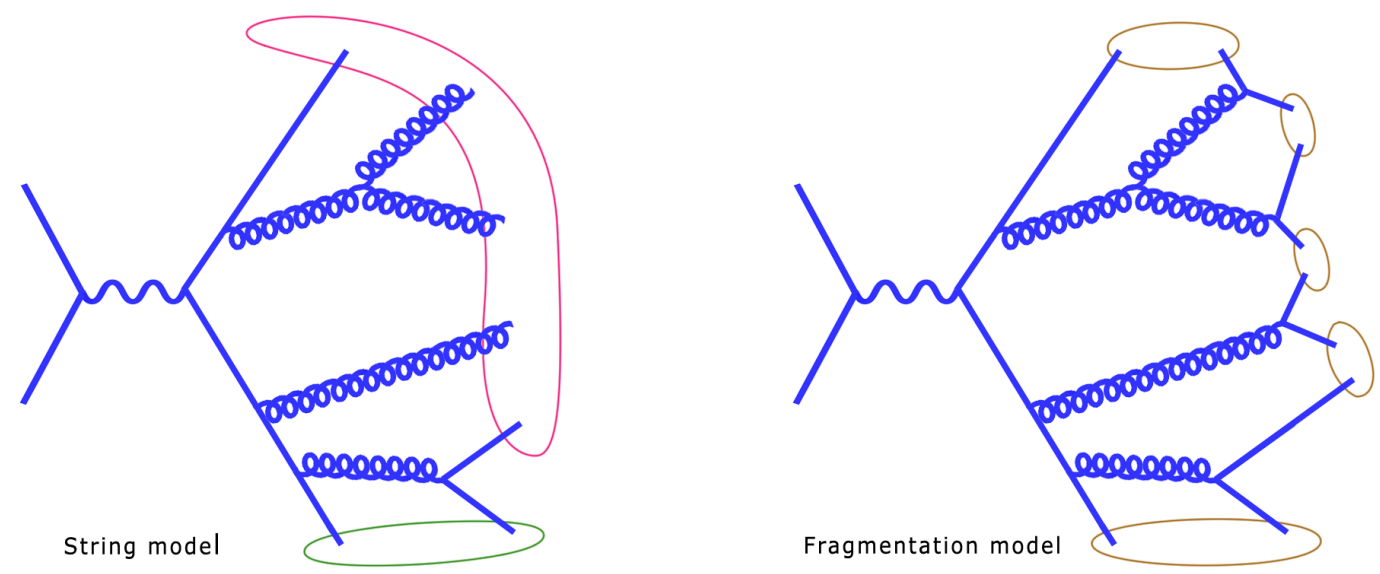

Figure 4.2. Left: The string fragmentation model diagram. The pink contour represents a string between two quarks kinked by the gluons and the green contour is a single straight string between two quarks. Right: The cluster fragmentation model diagram. The brown contours represent clusters formed by pairs of two quarks.

Since the string fragmentation model is not derived directly from QCD, it has many tunable parameters that need to be determined. However, the model does appear to be a good approximation. The process of hadronization is independent of how the hadronizing partons were produced, but does depend on the color connections from the final state partons. Once all free parameters are determined from the data, the model can be used to describe different type of collisions or collision at different energies.

The cluster fragmentation model is used in the HERWIG generator. In this model, the clusters made of two quarks are considered as basic elements for producing hadrons. Since the shower evolution results in all gluons decayed to light-quark pairs $q \bar{q}$, color singlet clusters $q \bar{q}$ can be formed in the nearest shower branches. Most of the clusters are low-mass. The massive clusters, however, can additionally produce $q \bar{q}$ pairs by a mechanism similar to the string fragmentation model. The newly produced quarks are 
also involved in the cluster formation process.

Unlike strings, clusters do not have any internal structure. They are characterized by mass and flavor content. The clusters are assumed to decay isotropically into two primary hadrons.

\subsection{Monte Carlo Samples}

To perform the top quark mass measurement with the method described in Chapter 7, the Monte Carlo samples for signal and background events were generated and processed through DØ detector simulation software. The following subsections detail Monte Carlo signal and background samples.

\subsubsection{Signal Sample}

The set of $t \bar{t}$ samples are simulated in the range of the top quark mass $140 \mathrm{GeV}$ $<m_{t}<200 \mathrm{GeV}$. In particular, the samples used in this thesis are generated with the following list of the top quark masses: 140, 145, 150, 160, 165, 170, 172, 175, 180, 185, 190, 195, and $200 \mathrm{GeV}$. To generate the parton hard scattering process, ALPGEN matrix element generator was used. Either a quark and an antiquark or two gluons were specified as interacting partons to compute matrix element to the leading order. The parton momenta are taken from the parton density distribution CTEQ6L1 [72]. The scattered parton showering (ISR/FSR) and hadronization are generated by PYTHIA.

To properly simulate events with high jet multiplicity, ALPGEN generates separate samples with different number of final state partons (0 and 1 light partons, and 2 or more light partons states). However, extra jets can be created in parton shower during hadronization process by PYTHIA. This effect is common when using ALPGEN in conjunction with PYTHIA. To keep correct jet multiplicity after the parton showering process is done, a special matching technique is applied. The matching prescription is 
called MLM matching [77]. According to this procedure, the event is rejected if the jet does not match a parton multiplicity. Every event is assigned an additional weight to account for rejected events and keep a hard scattering process cross-section accurate.

\subsubsection{Background Samples}

The main background processes for the $t \bar{t}$ dilepton channel originate from $Z$ boson or $W W / W Z / Z Z$ decaying into 2 leptons and jets produced from ISR/FSR. Background due to physics processes that give a signature very similar to the signal is called a physics background. As in the case of the signal, ALPGEN and PYTHIA are used to generate this $Z \rightarrow 2 l+$ jets background. The $W W / W Z / Z Z \rightarrow 2 l+$ jets background is fully generated by PYTHIA. Background due to misidentified electrons and muons can arise from instrumental effects and is called an instrumental background. For example, jets usually comprise leading $\pi^{0}$ that can decay producing an electron-positron pair. A conversion-produced track matching particles produced in $\pi^{0}$ decay can mimic an isolated electron with large $p_{t}$. An isolated muon may appear from the jet that is not reconstructed properly. Using sophisticated methods, instrumental background is derived from the data. 


\section{Chapter 5}

\section{DATA RECONSTRUCTION AND OBJECT IDENTIFICATION}

The data collected from $\mathrm{D} \varnothing$ is stored on tapes in a raw format. This raw format data contains only basic information from the detector, such as digitized charges or voltages, and therefore has to be additionally processed to become suitable for the subsequent physics analyses. For instance, raw data contains particle hits from the fiber tracking system, energy deposition in the calorimeter cells, hits in the muon system, trigger information etc. For the physics analyses to be performed, analog and digital signal information from the raw data needs to be converted into the physics objects (e.g. electrons, muons) with corresponding kinematic parameters (e.g. transverse momentum, energy, angles). The process of such computation is know as event reconstruction.

At the stage of the event reconstruction, high level software algorithms are utilized to reconstruct the events and identify physics objects. All algorithms are implemented in a specialized software framework that operates on the raw data. The following sections describe the event reconstruction chain as well as algorithms used at D $\varnothing$ to reconstruct main physics objects.

\subsection{Data Reconstruction}

The D $\varnothing$ Offline Reconstruction Program (d0reco) is a specialized framework developed by the DØ Algorithms group [79] and used at the experiment for reconstructing objects. It runs at the offline production farms and processes either the raw data from the detector or Monte Carlo simulated events. The framework employs a special data model of input and output information. The data model is known as the D $\varnothing$ Event 
Data Model (EDM). The information on the event is stored in the form of chunks. Primary input information to the doreco comes in chunks of the raw detector signals. The doreco output information also contains chunks of the information about reconstructed objects. There are two types of output formats that are optimized for size. The larger size data format is the Data Summary Tape (DST). DST contains all calculated information necessary for physics analysis. The smaller size data format is the Thumbnail (TMB). TMB contains only a subset of the DST information that is needed for physics analysis. The per-event size of TMB is about 10 times smaller than for DST. The offline reconstruction is comprised of several stages:

1. Detector-specific processing: at this stage individual detector data chunks are unpacked and raw information is associated with physical detector elements. Detector specific calibration is applied.

2. Track reconstruction: based on the output from the tracking detectors, several algorithms are used to reconstruct global charged particle tracks. Running track reconstruction algorithms requires intensive computing usage.

3. Vertex reconstruction: primary and secondary vertex candidates are sought at this stage. Primary vertex indicates the event's main interaction point and is used for calculation of various kinematical parameters. Secondary vertices, if found, indicate the presence of long-lived particle in the event.

4. Particle identification: using various sophisticated algorithms and the results derived at previous stages, physics object candidates are formed at this stage. Heavyquarks candidates and tau decays are identified using special algorithms for longlived particle reconstruction.

To be used in analyses at $\mathrm{D} \varnothing$, the output data from $d 0$ reco is converted into Common Analysis Format. A special software program called TMBAnalyze is utilized to produce 
ROOT trees in CAF format from the d0reco output. The data is then ready for physics analyses in CAF software framework.

\subsection{Object Identification and Reconstruction}

The following sections describe the details of reconstruction algorithms and identification criteria for such physics objects as tracks, vertices, electrons, muons, jets and missing transverse energy.

\subsubsection{Tracks}

A track is an important element for particle identification and momentum measurement. When a charged particle traverses the tracking system, it leaves hits in the different layers of the SMT and the CFT systems. Hits represent energy deposits in the certain tracking regions. For example, for the SMT a tracking region is represented by corresponding segment. Passing through the tracking system, the particle trajectory is bent by the magnetic field of the solenoid. Any given events may contain hundreds of individual particles that result in thousands of hits in the tracking system. Thus, successful particle track reconstruction from those hits requires application of sophisticated pattern recognition algorithms.

Two different algorithms are used at $\mathrm{D} \varnothing$ for track reconstruction: the Alternative Algorithm (AA) [80] and the Histogram Track Finder (HTH) [78]. The Alternative Algorithm is a very efficient approach for tracks with low $p_{T}$. It also has smaller rate of misidentified tracks when compared to the HTH technique. AA is commonly used at other experiments in High Energy Physics. However, the AA approach is not very well suited for high detector occupancies and large number of hits in the tracking system $\left(10^{4}-10^{6}\right)$. The HTH technique is based on the Hough transformation [81] approach and shows good results for tracks with high $p_{T}$. Originally, the HTH method was developed 
for machine analyses of photographs from bubble chamber experiments. At DØ both algorithms are executed sequentially to find all possible track candidates. All found track candidates are added to the list. Finally, a Kalman Filter algorithm [82] creates, combines and refits tracks from the list of track candidates.

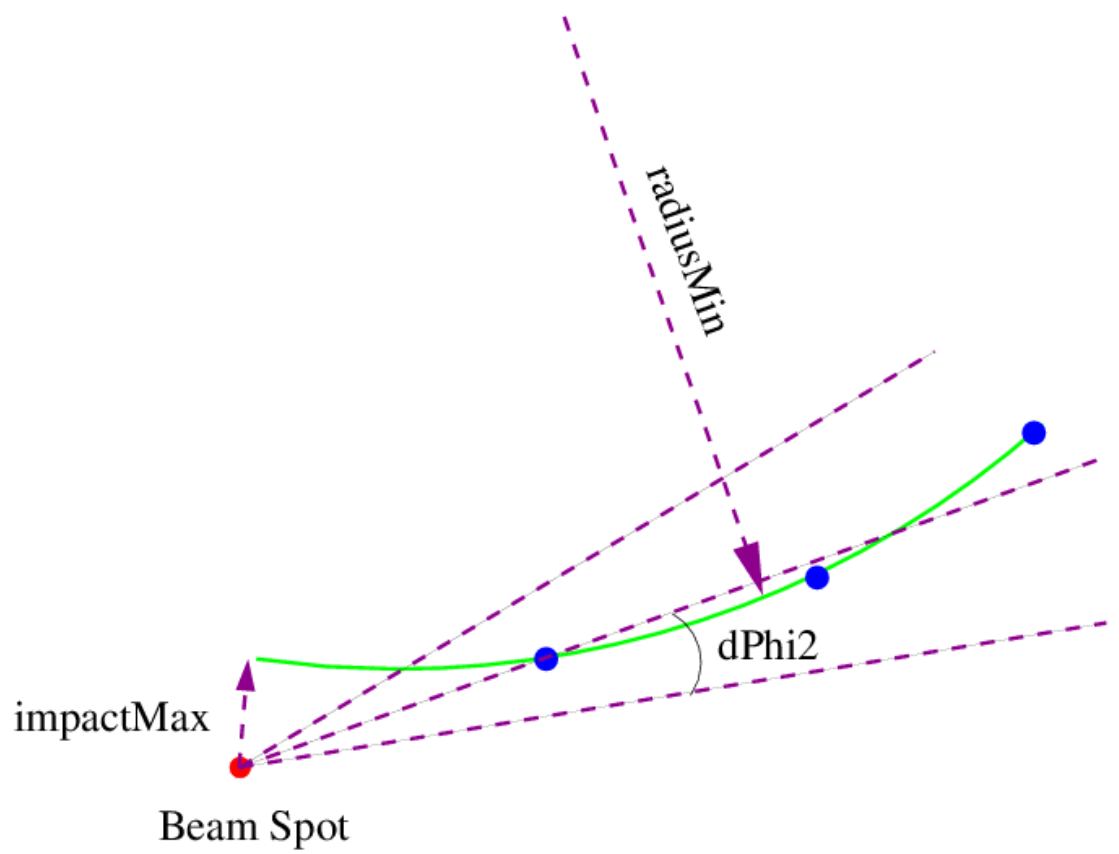

Figure 5.1. Track construction diagram using the Alternative Algorithm technique. The track candidate (green line) is based on the three consecutive hits (blue dots).

The AA method starts from a hit in the innermost layer of the SMT; then it searches the second hit in the next layer (see Figure 5.1). An axial angle between two hits as seen from the beamspot has to be less than a pre-set threshold $\Delta \phi<0.08$. After finding a second hit, the algorithm looks for a third hit in the next layer outward from the beam. The third hit is selected if the radius of reconstructed track candidate is less than $30 \mathrm{~cm}$. The requirement on the length of track radius corresponds to requirement on particle 
transverse momentum $p_{T}>180 \mathrm{MeV}$. Additionally, a distance of closest approach to the beam line has to satisfy $d_{0}<2.5 \mathrm{~cm}$ and $\chi^{2}$ of the track candidate has to be less than 16 .

Reconstruction continues to the next layers of the SMT or CFT for all track candidates found from the combination of three hits. The Kalman filter is used to reconstruct the rest of the track candidate. Based on knowledge of the material in the detector and the magnetic field, it extrapolates the track candidates to the next layers. If any additional hit is found in the track expectation region, the filter tries to associate the hit with the track candidate hypotheses. The hit is added to the track if the overall track candidate $\chi^{2}$ is still less than 16. If there are no hits found in three consecutive layers, then the algorithm stops. Otherwise the track reconstruction continues until the end of the detector is reached.

The trajectory of a charged particle moving in the homogeneous magnetic field projected onto the transverse plane is a circle. The HTH method uses the transverse projection of all the hits to reconstruct a track candidate. It employs a special parameter space $\left(\rho, \phi, d_{0}\right)$. Every circular track projection in the transverse plane can be described as a point in this parameter space; $\rho$ is the circle radius, $\phi$ is the azimuthal angle to the center of the circle, and $d_{0}$ is the distance of closest approach to the circle from the coordinate system origin, called an impact parameter. The parameter space is only two dimensional $(\rho, \phi)$. Each single hit can be described by an infinite number of circles passing through it (see Figure 5.2). Those circles form a straight line in the space $(\rho, \phi)$, and therefore the hit is also defined as a straight line in the parameter space. Subsequently, any two hits in the parameter space represent two intersecting straight lines. If several hits are from the same track, then in this parameter space, the set of straight lines from these hits will intersect at one point. The coordinates of that point will define the parameters of the track. 
In practice, the two-dimensional parameter space is represented by a two-dimensional ROOT histogram. The algorithm considers a different pair of the hits and populates this histogram. All of the hits belonging to the same track produce a peak in the histogram at a certain bin. Hits from different tracks produce randomly populated histograms. The track candidate parameters $\left(\rho_{0}, \phi_{0}\right)$ are defined from the location of the peak.

\subsubsection{Primary Vertex}

The point at a which hard-scattering process occurs in $p \bar{p}$ collisions is known as the primary vertex $(\mathrm{PV})$. All outgoing particles from such an inelastic interaction originate from the primary vertex. Therefore to measure kinematic parameters of resulting particles with good precision, a precise determination of the primary vertex location is very important. Additionally, the PV location allows us to discriminate against particles that do not belong to the event of interest. For example, particles coming from cosmic rays can be excluded from the event as they do not originate from the PV.

At the beam luminosities at the Tevatron, there are on average two PV in each event. Some events may contain many more PVs. For instance, to calibrate jet energies at D $\varnothing$, events are modeled with up to $20 \mathrm{PVs}$ in the event. The location of the PVs in the transverse $(\mathrm{x}-\mathrm{y})$ plane may differ by up to $40 \mu \mathrm{m}$ between events. The position along the $z$-axis is distributed roughly according to a Gaussian distribution with a standard deviation of about $28 \mathrm{~cm}$.

One of the main problems of vertex reconstruction is to discriminate primary vertex tracks from secondary vertex tracks. This is especially critical at high luminosities and for particles with short lifetime. An additional challenge is to exclude minimum bias tracks that are very close to primary vertex.

An adaptive fitting technique [83] is employed at D $\varnothing$ for primary vertex reconstruction. Comparing to standard pattern recognition systems, the adaptive fitting is designed 
to deal with misidentified tracks and tracks originated from different interactions. The technique does not apply a "hard" cut to reject bad tracks. Instead, all tracks are assigned different weights with which they contribute to the primary vertex. Weights are assigned in such a way that tracks closer to the PV in the transverse plane have larger contribution to the vertex than tracks with larger impact parameter. The adaptive fitting track reconstruction and identification consists of three stages: track selection, vertex fitting and vertex selection.

Track selection requires a track to have $p_{T}>0.5 \mathrm{GeV}$ and two or more hits within the acceptance region of the SMT. A special algorithm is used to cluster tracks belonging to different interaction points. This algorithm gathers tracks that are within $2 \mathrm{~cm}$ from each other along the $z$-axis. The algorithm is therefore called as $z$-clustering.

The vertex fitting is performed separately for every cluster. The pre-selection stage consists of two steps. At the first step, a Kalman filter algorithm is applied. It starts from fitting tracks to find their common vertex. The track with the highest contribution to the fit $\chi^{2}$ is excluded, if the fit $\chi^{2}$ per degree of freedom is bigger than 10. Track exclusion repeats iteratively until the $\chi^{2}$ per degree of freedom is less thn or equal to 10. At the second step, tracks are pre-selected according to their impact parameter relative to the vertex spot determined in the previous step. To properly take into the account the track impact parameter and vertex spot error, a special quantity called impact parameter significance is defined. The significance is $d_{0} / \sigma\left(d_{0}\right)$, where $d_{0}$ is the track impact parameter (i.e. closest distance from the vertex spot to the track) and $\sigma\left(d_{0}\right)$ is the impact parameter uncertainty. The vertex fitting pre-selection requires the $d_{0} / \sigma\left(d_{0}\right)<5$.

After the pre-selection, the vertex fitting is performed using the Adaptive vertex fitting algorithm. The main idea of the algorithm is to reduce the effect from distant tracks in the vertex fit. The algorithm is also based on the Kalman filter. We obtain 
an additional improvement by assigning track errors a weight by sigmoidal function according to their contribution to the vertex:

$$
w_{i}=\frac{1}{1+e^{\left(\chi_{i}^{2}-\chi_{\text {cutoff } f}^{2}\right) / 2 T}}
$$

where $\chi_{i}^{2}$ is the $\chi^{2}$ contribution from the $i$-th track, $\chi_{\text {cutoff }}^{2}$ is a cutoff constant equal 10, $T$ is the parameter that controls function sharpness.

As at the pre-selection stage, the Kalman filter is used iteratively:

1. The tracks are fitted with initial weight equal 1 . The new weights $w_{i}$ are computed according to the formula 5.1

2. Each track is weighted with its $w_{i}$ and the tracks are fitted again. The weights are re-computed again.

3. The tracks with weights $w_{i}<1 \cdot 10^{-6}$ are removed from the next fitting iteration.

The algorithm iterates until convergence conditions are satisfied: $\left|w_{i}-w_{i-1}\right|<1 \cdot 10^{-4}$ and the number of iterations is less than 100.

Having found all vertices with the help of the Adaptive fitting technique, the following goal is to separate hard-scattering vertices and vertices coming from minimum bias interactions. Based on the fact that minimum bias tracks typically have lower transverse momentum than hard-interaction tracks, every track is assigned some probability to be originated from a minimum bias vertex. A special probabilistic approach [84] is employed at D $\varnothing$ to determine minimum bias probability for each track. The minimum bias probability for the vertex is then calculated by multiplying all individual track minimum bias probabilities. Ultimately, the vertex with the lowest probability of originating from a minimum-bias interaction is taken as the hard-interaction primary vertex.

\subsubsection{Electrons}


The presence of an isolated electron in the event is an important physics signature for many analyses. Such analyses include the top quark cross section and mass measurements, precision measurements in the electroweak sector, searches for new physics, etc. It is also crucial to be able to distinguish real isolated electrons from misidentified ones (fakes). Thus efficient electron identification and fake rejection techniques were developed and successfully implemented at D $\varnothing$.

The electron identification uses information from the calorimeter and the tracking system. It starts from finding energy clusters in the EM layers of the calorimeter. Although photons leave very similar signal in the EM layers, they don't have a matching track in the tracking system as is the case with electrons. Electron quality criteria are further applied to separate electrons from hadrons.

\subsubsection{Cluster Reconstruction and Requirements}

Cluster reconstruction begins from finding EM towers in the calorimeter. Every tower is defined as a group of cells with the same $\eta$ and $\phi$ coordinates. Cells contributing to a tower are from the four EM layers and the first hadronic layer. A tower is typically $0.1 \times 0.1$ in $\eta \times \phi$ space, and the minimum transverse energy deposited has to be at least $50 \mathrm{MeV}$. After finding all towers, an EM cluster is defined using the simple cone algorithm. All adjacent EM towers in the cone with radius $R=0.4$ are grouped together to form a cluster around a tower with the highest $E_{T}$.

The following requirements are applied to select electron-like EM clusters:

- Transverse energy. The transverse energy of a cluster is required to be greater than $1.5 \mathrm{GeV}$.

- Electromagnetic fraction. The electromagnetic fraction is defined as 


$$
f_{E M}=\frac{E_{E M}}{E_{T O T}}
$$

where $E_{E M}$ is the energy deposited in the electromagnetic calorimeter, and $E_{T O T}$ is the total energy deposited in the calorimeter (all EM and hadronic layers). The cluster is required to have $f_{E M} \geq 0.9$.

- Isolation. The isolation fraction is defined as

$$
f_{i s o}=\frac{E_{T O T}(0.4)-E_{E M}(0.2)}{E_{E M}(0.2)}
$$

where $E_{T O T}(0.4)$ is the total energy inside a cone with radius $R=0.4$, and $E_{E M}(0.2)$ is the energy in the EM layers only inside a cone with radius $R=0.2$. The cluster isolation requirement is $f_{\text {iso }} \leq 0.15$.

These requirements provide high efficiency for identifying real electrons while substantially suppressing the main backgrounds: $\pi^{0}$ decay into two photons when $\pi^{0}$ is belongs to a jet, and early photon conversion into electron-positron pair. Additionally, the isolation fraction requirement significantly reduces the background from semileptonic decay of $b$ and $c$ quarks.

\subsubsection{Electron Quality Variables}

By imposing additional quality requirements, a further background reduction can be achieved. Those requirements include matching a track to the cluster, comparison of the shower shape of the cluster with that expected from the electron, and a likelihood calculation of the electron to originate from background processes.

The following quality requirements are imposed on each electron candidate:

- Track-match $\chi^{2}$. As a charged particle passing through the detector, an electron leaves a track in the tracking system. This electron cluster must have at least one 
reconstructed track that matches the cluster. The track-match $\chi^{2}$ is defined as follows:

$$
\chi_{\text {track-match }}^{2}=\left(\frac{\Delta \phi}{\sigma_{\Delta \phi}}\right)^{2}+\left(\frac{\Delta z}{\sigma_{\Delta z}}\right)^{2}+\left(\frac{E_{T} / p_{T}-1}{\sigma_{E_{T} / p_{T}}}\right)^{2}
$$

where $\Delta z$ and $\Delta \phi$ are the difference between the track position and the EM cluster position at the third layer of the calorimeter; $E_{T} / p_{T}$ is the transverse energy of the EM cluster as measured by the calorimeter divided by the transverse momentum of the track; $\sigma_{x}$ is the experimental resolution of variable $x$. For every available track, the probability $P\left(\chi_{\text {track-match }}^{2}\right)$ is computed. $P\left(\chi_{\text {track-match }}^{2}\right)$ reflects how probable that a given track is matched to the cluster. The track with the highest probability is chosen as the electron track.

- H-matrix $\chi^{2}$. The longitudinal and transverse shape of an electron shower in the calorimeter is different from that of a jet. This difference in shower development can be used to discriminate an electron from a jet. The following seven variables are used to parameterize shower shape of the cluster: the energy deposition in each of the first $4 \mathrm{EM}$ layers of the calorimeter, the cluster size in the third layer of the calorimeter, the logarithm of the total energy, and the $z$ position of the primary vertex divided by its uncertainty. To compare shower shape of the cluster with shower shape for an electron, the H-matrix technique [85] is used. The Hmatrix can be determined from the covariance matrix $M$, namely $H \equiv M^{-1}$. The covariance matrix, in turn, is defined from the sample of Monte Carlo electrons as follows:

$$
M_{i j}=\frac{1}{N} \sum_{n=1}^{N}\left(x_{i}^{n}-\bar{x}_{i}\right)\left(x_{j}^{n}-\bar{x}_{j}\right)
$$

where $\mathrm{N}$ is the total number of electrons in the sample, and the $x_{i}^{n}$ is the $i$ th variable for the $n$-th particle. The parameter that measures the degree of 
agreement between shower shape of the cluster and that expected for an electron is calculated as:

$$
\chi_{H 7}^{2}=\sum_{i, j=1}^{7}\left(x_{i}-\bar{x}_{i}\right) H_{i j}\left(x_{j}-\overline{x_{j}}\right)
$$

To identify a cluster as an electron, we require $\chi_{H 7}^{2}<50$.

- Electron Likelihood. In order to further improve electron purity, the likelihood approach [86] is employed. The electron likelihood is based on several input variables and trained using $Z \rightarrow e^{+} e^{-}$data for the signal and $\mathrm{QCD}$ jet events for the background. The input variables used for the likelihood are:

- Number of tracks in $\Delta R<0.4$

- Scalar sum of track $p_{T}$ in hollow cone $0.05<\Delta R<0.4$

- Spatial track-match probability $P\left(\chi_{\text {track-match }}^{2}\right)$

- Isolation fraction $f_{\text {iso }}$

- Electromagnetic fraction $f_{E M}$

- Ratio of the transverse energy of the cluster in the calorimeter to the transverse momentum of the matched track, $E_{T}(E M c l u s t e r) / p_{T}($ track $)$

- Distance of closest approach $(D C A)$ of the cluster track candidate with respect to the primary vertex in the transverse plane.

- H-matrix parameter $\chi_{H 7}^{2}$

- H-matrix parameter $\chi_{H 8}^{2}$. The $\chi_{H 8}^{2}$ parameter additionally to the quantities used in $\chi_{H 7}^{2}$ employs the transverse width of the shower variable.

Consequently, distributions of each variable are obtained from Monte Carlo signal and background samples. The distribution of each variable $x_{i}$ is used to assign a 
probability for the cluster to be a signal or background: $P_{s i g}\left(x_{i}\right), P_{b k g}\left(x_{i}\right)$. Multiplying the probabilities for different variables, the overall probability to be signal or background can be obtained under the assumption that the variables are not correlated:

$$
\begin{aligned}
P_{s i g}(\mathbf{x}) & =\prod_{i} P_{s i g}\left(x_{i}\right) \\
P_{b k g}(\mathbf{x}) & =\prod_{i} P_{b k g}\left(x_{i}\right)
\end{aligned}
$$

Then the electron likelihood can be constructed as:

$$
L(\mathbf{x})=\frac{P_{s i g}(\mathbf{x})}{P_{s i g}(\mathbf{x})+P_{b k g}(\mathbf{x})}
$$

When $L(\mathbf{x})$ is close to 1 then the cluster is deemed to be from an electron. If $L(\mathbf{x})$ is close to 0 , this corresponds to the background. The electrons used in this thesis are required to have $L(\mathbf{x})>0.85$.

\subsubsection{Electron Energy Scale}

The $Z$ boson mass is well-known parameter, measured to be $91.1875 \pm 0.0021 \mathrm{GeV}$ [8]. The invariant mass of two electrons in D $\varnothing$ data from $Z \rightarrow e^{+} e^{-}$decays does not agree with this value. The cause of the lower mass originates from different electronics effects in the calorimeter that need to be accounted.

Test-beam studies showed that the relationship between true and measured electron energies has a linear form:

$$
E^{\text {true }}=\alpha E^{\text {meas }}+\beta
$$


where $\alpha$ is called electron energy scale and $\beta$ is the offset. The parameters $\alpha$ and $\beta$ can be obtained from measured dielectron masses of the known particles that decay into electrons: $Z$ boson, $J / \psi$ and $\pi^{0}$.

\subsubsection{Muons}

The $\mathrm{D} \varnothing$ detector has an ability to identify muons in a few different ways involving independent detector subsystems. However, the main component in muon identification and muon measurement is the three layer muon system.

A muon, passing through the muon system, leaves a signal in the form of hits in every layer. The hits can be subsequently grouped together and form a muon track segment. A muon track segment in a layer is reconstructed by fitting a straight line to the hits in that layer. It is possible to have more than one muon track segment found in any given layer. In such case a segment with the best fit $\chi^{2}$ is used.

After track segments are found in all three layers, a muon track reconstruction is performed by fitting a curve through the segments in every layer. To properly reconstruct a muon track, the track fitting procedure accounts for the effect of the toroid magnetic field between layer A and B as well as for the effects due to substantial presence of the material between those layers. To determine a muon momentum, the curvature of reconstructed track is used.

In practice, muon reconstruction uses information from both the muon detector and the central tracker. The central tracker provides muon momentum measurement with a much higher precision than the muon detector. Additionally, it is also very effective at reconstructing tracks in the whole angular acceptance of the muon detector.

Once a muon track is reconstructed by the muon detector, it must be matched to a track in the central tracking system. The track matching is performed by extrapolating the muon track through the calorimeter to a track in the central tracker. The track 
matching procedure properly accounts for possible energy loss and multiple scattering in the calorimeter. The degree of agrement between two tracks is described by a $\chi^{2}$ of the muon track fit to a central track.

The level of agreement given by the $\chi^{2}$ of the muon track fit is used in assessing muon quality. If these tracks are in good agreement with each other and a muon is considered of a good quality then its momentum is concluded from the central tracker. If the $\chi^{2}$ shows bad fit agreement then this may be an indication of having a cosmin muon passing through the detector.

\subsubsection{Muon Quality Variables}

Muon quality variables as described in [87] are used to classify muon candidates. There are four quality variables: muon quality, tracking quality, isolation and cosmic veto. Depending on the type of the analysis, different values of the quality variables can be required. Usually higher requirements provide a better quality of muons but tend to have lower reconstruction efficiency. Analyses that are less sensitive to the quality of muons can apply less strict requirements to quality variables.

In general muon quality variables are detailed below:

- Muon Type and Quality. To evaluate quality of muon candidates, two parameters are used : muon type and muon quality. The muon type parameter is described by the special variable $n s e g$. If $n s e g$ has a positive value, i.e. $n s e g>0$, then the muon reconstructed in the muon detector matches a track in the central tracker. The case when $n s e g<0$ indicates that there is no central track that matches the muon registered in the muon system. The absolute value of $n s e g$ tells how exactly the muon was registered in the muon detector:

$-|n s e g|=1$. The muon is registered with A-layer hits only.

$-|n s e g|=2$. The muon is registered with B or C-layer hits only. 
$-|n s e g|=3$. The muon is registered in all three layers.

The muon quality parameter defines three types of muons: "Loose", "Medium" and "Tight". To assign this value, the hit information from the muon system and the nseg variable are used. All "Tight" muons require $|n s e g|=3$. If muon has $|n s e g|=3$ but fails "Tight" hit requirements, it can be marked as "Medium" or "Loose". In all cases when $|n s e g|<3$, depending on the hit information, the muon is labelled either as "Medium" or "Loose". Detailed information on muon quality parameter definition is given in Appendix A.

- Track Quality. Analogous to the muon quality three types of tracks have been defined: "Loose", "Medium" and "Tight". The track quality assessment is based on the following parameters:

- Number of hits either in the SMT or CFT system

$-\chi^{2}$ per degree (or $\chi^{2} /$ d.o.f.) of freedom of the central track fit

- Distance of closest approach $(D C A)$ of the track with respect to the primary vertex

The muon track quality results are defined as follows:

- "Loose track". $|D C A|<0.2 \mathrm{~cm}$. If the track also contains a hit in the SMT then the requirement is changed to $|D C A|<0.02 \mathrm{~cm}$.

- "Medium track". The Medium track must satisfy Loose track requirements and additionally pass a $\chi^{2} /$ d.o.f. $<4$ requirement.

- "Tight track". The Tight track add SMT hits to the requirement for Medium tracks.

Most of the cosmic muon tracks do not pass the $D C A$ track criterion. Such muons are not originated from the collision point and therefore have very large $D C A$ 
value. The muons that come from hadronic decay (e.g. $\pi \rightarrow \mu \overline{\nu_{\mu}}$ ) or semileptonic decay of heavy quarks (e.g. $b \rightarrow W u \rightarrow \mu \overline{\nu_{\mu}} u$ ) are rejected by the $\chi^{2}$. Long-lived particles, like hadrons or heavy quarks, usually travel substantial distance from the interaction point before decaying and therefore the fit if muon central track results in large $\chi^{2}$.

- Muon Isolation. Muons produced due to heavy flavor decay tend to have a small opening angle. As the opposite to that, interesting physics processes often include $W$ or $Z$ bosons that decay into muons with very wide opening angle. This suggests the muon isolation criterion to be an important requirement in separating interesting physics muons from heavy flavor background. There are five variables that are used in muon isolation requirements:

- TrackHalo $=p_{T}^{\text {tracks }}(0.5)-p_{T}^{\mu}$

- CalorimeterHalo $=E_{T}^{\text {cells }}(0.4)-E_{T}^{\text {cells }}(0.1)$

- ScaledTrackHalo $=$ TrackHalo $/ p_{T}^{\mu}$

- ScaledCalorimeterHalo $=$ CalorimeterHalo $/ p_{T}^{\mu}$

- $\Delta R(\mu, j e t)$ - distance to the closest jet in $\eta$ - $\phi$ plane

where $p_{T}^{\text {tracks }}(0.5)$ is the sum of the transverse momentum of all tracks inside a cone of radius $0.5, p_{T}^{\mu}$ is the transverse momentum of a muon; $E_{T}^{\text {cells }}(0.4)$ and $E_{T}^{\text {cells }}(0.1)$ are the transverse energy deposited to the calorimeter cells that lie around the muon track inside a cone of radius 0.4 and 0.1 correspondingly. Various isolation requirements can be produced by combining different isolation variables. Every particular combination of variables with corresponding cut values is called an isolation working point. The set of the main isolation working points is given in Appendix B. 
- Cosmic Veto. Cosmic muons that have passed DCA requirement and thus can be misidentified as muons from the collision point are also required to satisfy a cosmic veto parameter. This parameter uses the scintillator hit times to distinguish good muons (resulting from the collision) from cosmic muons. The scintillators provide precise timing information with response time of $1.6 \mathrm{~ns}$. This allows selection of muons that have a timestamp matching the collision time with very high efficiency. Subsequently, muons that do not match the collision time are marked as cosmic ray and are therefore rejected. For every layer in the muon detector, the cosmic veto parameter defines a time interval within which a muon is considered to be a muon coming from the $p \bar{p}$ collision:

$$
\begin{aligned}
& \text { - A-layer }|\Delta t|<10 \mathrm{~ns} \\
& \text { - B-layer }|\Delta t|<10 \mathrm{~ns} \\
& \text { - C-layer }|\Delta t|<10 \mathrm{~ns}
\end{aligned}
$$

\subsubsection{Jets}

There is no unique definition of a jet, unlike the case of elementary particles such as an electron, muon and a photon. This is due to the fact that a jet is not a single elementary particle but rather a spray of different particles, elementary and not, moving along some direction with relatively small opening angle. In a simple way, a jet can be defined as a shower of particles seen by the detector in some narrow cone.

Most of the particles in a jet are a result of showering and hadronization processes of a quark or a gluon. Because of the "confining" nature of the strong interaction, particles that carry a color charge - quarks and gluons - can never appear in free form. Instead they shower and subsequently fragment into a hadrons before being detected in the detector. The showering and fragmentation of a parton from hard scattering interaction or ISR/FSR processes produces hadrons moving in close vicinity to the direction of 
the original parton. Such parton evolution to many hadrons, ultimately, is seen in the detector and defined as a jet.

Additionally, some of the hadrons can decay into other particles before reaching the detector. The hadron collection includes substantial amount of neutral pions. Subsequently, most of the pions may easily decay into two photons $(\pi \rightarrow \gamma+\gamma)$ that can be seen in the electromagnetic layers of the calorimeter. Less frequently a pion can decay into electrons $(\pi \rightarrow \gamma+e+e)$ or a muon $(\pi \rightarrow \mu+\nu)$. Thus the jet content is not limited to hadrons only, it can also contain electrons, muons and photons.

Jet showers can differ dramatically in shape and particle content. Jets deposit energy both in the electromagnetic and hadronic layers of the calorimeter. Complementary to that, jets usually have many associated tracks from the central tracking system.

Special algorithm and quality requirements have been developed at $\mathrm{D} \varnothing$ to reconstruct and identify jets in the detector. The following subsections describe these in detail. The jet energy calibration is detailed in Chapter 6 .

\subsubsection{Jet Reconstruction}

To design an efficient jet reconstruction algorithm, certain theoretical and experimental requirements should be satisfied. Thus from an experimental standpoint, the ideal jet algorithm must satisfy the following main requirements:

- Jet finding technique should be insensitive to multiple hard scattering processes at high luminosity

- All interesting jets in the event should identified by the algorithm. For the purposes of my analysis, the jet is considered to be an interesting if it arises from an energetic parton as a result of hard scattering process

- The effects of resolution smearing and angle biases should not be amplified by the 
algorithm

- The algorithm performance should be independent of the detector

Theoretical requirements include insensitivity to soft gluon (low $p_{T}$ ) radiation, invariance under boosts, infrared safety, collinear safety and others. The full list of requirements for the ideal jet algorithm is described in detail in [88].

At DØ, the RunII Cone Algorithm [89] is employed for finding and reconstructing jets in the event. The cone algorithm is designed to implement the ideal jet reconstruction algorithm as closely as possible. The main idea behind the cone algorithm is to find a jet by surrounding the shower of particles with a cone of certain size in $\eta \times \phi$ space. The cone algorithm uses a fixed geometry approach to isolate dominant energy flow in the calorimeter and thus reconstruct a jet.

The pre-reconstruction stage starts with cells in the calorimeter. All noisy cells are removed using the T42 algorithm [90] before the algorithm runs. Each cell is assumed to have zero mass, and has the four-momentum $p^{\text {cell }}=\left(E^{\text {cell }}, \vec{E}^{\text {cell }}\right)$, where $E^{\text {cell }}$ is the energy measured in the cell, $\vec{E}^{\text {cell }}$ points from the primary vertex to the center of the cell. The cells are organized together to form towers in the calorimeter. Each tower has the four-momentum

$$
p^{\text {tower }}=\sum_{i=1}^{N_{\text {cells }}} p_{i}^{\text {cell }}
$$

where $p_{i}^{\text {cell }}$ is the four-momentum of the $i$-th cell and $N_{\text {cells }}$ is the total number of cells in the tower.

The actual RunII Cone Algorithm proceeds in three stages. The first stage is called clustering. At this stage the calorimeter towers are grouped into preclusters. The towers with $p_{T}^{\text {tower }}>0.5 \mathrm{GeV}$ are marked as seeds and the list of seeds is populated by the algorithm from the towers that pass the above criterion. The seed with highest $p_{T}^{\text {tower }}$ is 
surrounded with the cone of radius $\Delta R=0.3$. All towers in the cone are considered as belonging to one precluster with the four-momentum

$$
p^{\text {precluster }}=\sum_{j=1}^{N_{\text {towers }}} p_{j}^{\text {tower }}
$$

where $p_{j}^{\text {tower }}$ is the four-momentum of the $j$-th tower and $N_{\text {towers }}$ is the total number of towers forming the precluster. If any tower from the list of seeds appears also inside the cone, then it is removed from the list of seeds. An updated list of seeds is defined and the procedure repeats iteratively with the next leading seed until all preclusters are found.

At the second stage, protojets are formed from the preclusters. The list of preclusters serves as the list of seeds for making protojets. The algorithm adds a precluster to the list of seeds if $p_{T}^{\text {precluster }}>1.0 \mathrm{GeV}$ and there are more than one tower in the precluster. The precluster with highest $p_{T}^{\text {precluster }}$ from the list of seeds is surrounded with the cone of radius $\Delta R=0.5$. All preclusters in the cone are added together and form a protojet candidate. The center of the protojet candidate is determined by calculating a centroid from preclusters weighted by their $p_{T}^{\text {precluster }}$. If the distance between the initial seed and the center of the protojet candidate is less than critical value $\Delta R<0.001$, the protojet candidate is marked as a stable protojet. Otherwise, the center of the protojet candidate is used as the new seed and the procedure repeats until the stable protojet is found. The stable protojet is added to the list of protojets unless another protojet already exists in the list for which the following conditions are true $-\left|p_{T}^{P C} / p_{T}^{P J}-1\right|<1 \%$ and $\Delta R(P C, P J)<0.005$. Here, $\Delta R(P C, P J)$ is the distance between the protojet candidate and the protojet in $\eta \times \phi$ space, $p_{T}^{P C}$ and $p_{T}^{P J}$ transverse momentum of the protojet candidate and the protojet candidate respectively,. The protojet finding procedure is repeated until all protojets are found. The four-momentum of a protojet is given by 


$$
p^{\text {protojet }}=\sum_{k=1}^{N_{\text {preclusters }}} p_{k}^{\text {preclusters }}
$$

where $p_{k}^{\text {protojet }}$ is the four-momentum of the $k$-th protojet and $N_{\text {preclusters }}$ is the total number of preclusters in the cone of radius $\Delta R=0.5$ around the center of the protojet.

The third stage is merging and splitting of the protojets. The algorithm starts from the highest $p_{T}$ protojet selected from the list of protojets. The protojet becomes a jet and is removed from the list if it does not overlap with any other protojet. If the protojet shares more than $50 \%$ of $p_{T}$ with another protojet then the two are merged. The two overlapping jets are split if the shared $p_{T}$ fraction is less than $50 \%$. Merging is done by combining preclusters of the two neighbor protojets in one, and running the protojet reconstruction algorithm on the newly formed precluster to find a stable jet. Splitting is done by assigning all shared towers to the closest of the two protojets in $\eta \times \phi$ space. Merging and splitting process continues until all stable, not overlapping jets are found. The four-momentum of a jet is equal to the four-momentum of the stable protojet it corresponds to, e.g. $p^{\text {jet }}=p^{\text {protojet }}$. When merging and splitting of the protojets is done, jets with $p_{T}<6 \mathrm{GeV}$ are considered unphysical and therefore removed.

\subsubsection{Jet Quality Variables}

Often large noise fluctuations in the calorimeter can be misreconstructed as a jet by the reconstruction algorithm. However, these jets have no tracks associated and are therefore considered to be non-physical jets. Such jets are referred as fakes. In order to reduce the amount of fake jets, the following quality requirements [91] have to be applied :

- Electromagnetic fraction. To separate a jet from electromagnetic particles like an electron or a photon, the EM quality variable cut is placed. The electromagnetic fraction of a jet is required to be within the interval $f_{E M} \in[0.05,0.95]$. The EM 
fraction is defined as $f_{E M} \equiv \frac{E_{E M}}{E_{T O T}}$. Here, $E_{T O T}$ includes energy deposits from all (EM, FH and $\mathrm{CH}$ ) layers of the calorimeter.

- Coarse hadronic fraction. The coarse hadronic fraction is defined as

$$
f_{C H}=\frac{E_{C H}}{E_{T O T}}
$$

where $E_{C H}$ is the energy deposited in the coarse hadronic layers of the calorimeter. To remove the fakes from high level noise in the calorimeter, the following requirements are placed on $f_{C H}$ for different regions in $\eta$ :

$-|\eta|<0.8: f_{C H}<0.44$

$-0.8<|\eta|<1.5: f_{C H}<0.6$ for narrow jets, and $f_{C H}<0.4$ for wide jets

$-1.5<|\eta|<2.5: f_{C H}<0.46$

- Hot Cell Removal. The ration of the energy in the second most energetic cell is required to be ten times less than energy deposited in the the most energetic cell This removes the jets due to electronic noise.

- Hot Tower Removal. Any tower belonging to a jet cannot have more than $90 \%$ of its energy. If a jet has more than $90 \%$ of energy deposited to a single tower, it's likely to be from the noise in the readout electronics.

- Level 1 Trigger Confirmation. The jet is required to be confirmed by the Level 1 trigger. As defined in [92], the Level 1 ratio is

$$
L 1_{\text {ratio }}=\frac{p_{T}^{L 1}}{p_{T}^{\text {reco }}\left(1-f_{C H}\right)}
$$

where $p_{T}^{L 1}$ is the scalar sum of the transverse momentum of trigger towers inside a cone of radius $\Delta R=0.5, p_{T}^{r e c o}$ is the momentum of the reconstructed jet, $f_{C H}$ is the coarse hadronic fraction. The Level 1 trigger information contains only the 
100 hottest calorimeter towers of $0.2 \times 0.2$ in $\eta \times \phi$ space, and excludes the coarse hadronic layer. A jet is required to fulfill one of following Level 1 ratio cuts:

$$
\begin{aligned}
& -L 1_{\text {ratio }}>0.5 \\
& -L 1_{\text {ratio }}>0.35 \text { for jets with } p_{T}<15 \mathrm{GeV} \text { and }|\eta|>1.4 \\
& -L 1_{\text {ratio }}>0.1 \text { for jets with } p_{T}<15 \mathrm{GeV} \text { and }|\eta|>3.0 \\
& -L 1_{\text {ratio }}>0.2 \text { for jets with } p_{T} \geq 15 \mathrm{GeV} \text { and }|\eta|>3.0
\end{aligned}
$$

\subsubsection{Missing Transverse Energy}

The momentum conservation law states that the total transverse momenta before and after the collision have to be equal. Since the colliding proton-antiproton beams do not have any transverse momentum to the beam, the total transverse momentum of all particles produced in an event also has to equal zero. This implies a perfect balance of the momenta in the transverse plane of all particles in the event.

An important caveat arises because neutrinos traverse the detector without any interaction with its subsystems and thus appear completely unregistered. The presence of a neutrino in the final state leads to an imbalance in the sum of measured momenta. For historical reason, since an imbalance of total momenta in transverse plane is measured in the calorimeter, it is often referred as missing transverse energy, and represented by a symbol $E_{T}$. Missing $E_{T}$ is defined as the negative sum of the transverse momenta of all particles observed in the detector.

The reconstruction of missing $E_{T}$ takes place in the electromagnetic and fine hadronic layers of the calorimeter. The uncorrected missing energy is calculated as:

$$
\vec{H}_{T}^{\text {uncorr }}=-\sum_{i=1}^{N_{\text {cells }}} \vec{p}_{T i}
$$


where $\vec{p}_{T i}$ is a transverse momentum vector of the $i$-th cell, $N_{\text {cells }}$ is the number of cells in the calorimeter except the coarse hadronic layers. There is substantial level of noise in the coarse hadronic cells and therefore those are not included. To reduce the noise in the rest of the calorimeter, the T42 algorithm is used [90].

Due to the correction that photons, electrons and jets acquire during the offline reconstruction stage, missing $E_{T}$ has to be corrected as well. Additionally, missing $E_{T}$ needs to be corrected for muons, if those are present in the event. Since muons leave just a little signal in the calorimeter, their transverse momentum calculated by the central tracker is excluded from missing energy. The corrected missing energy is given by

$$
\vec{E}_{T}^{\text {corr }}={\overrightarrow{E_{T}}}_{T}^{\text {uncorr }}-\sum_{i=1}^{N_{\text {muons }}} \vec{p}_{T i}-\sum_{j=1}^{N_{\text {objects }}}\left(\vec{p}_{T j}^{\text {corr }}-\vec{p}_{T_{j}}^{\text {uncorr }}\right)
$$

where $i$ runs over all muons in the event, and $j$ runs over all other objects except of muons (i.e photons, electrons and jets); correspondingly $N_{\text {objects }}$ is the total number of objects in the event excluding muons, and $N_{\text {muons }}$ is the total number of muons in the event. $\vec{p}_{T j}^{\text {corr }}$ and $\vec{p}_{T j}^{\text {uncorr }}$ are the corrected and uncorrected momenta of each object. Detailed missing $E_{T}$ calculations and corrections are described in [93].

\subsubsection{Missing Transverse Energy Significance}

The momentum resolution of the objects in the event, photons, electrons, muons and jets, defines the corresponding resolution of missing $E_{T}$. It is not unlikely to obtain $E_{T}>0$ in the events due to measurement fluctuations. Thus, to evaluate how likely a non-zero value of the measured missing $E_{T}$ comes from the effect of resolution, a special variable, Missing Transverse Energy Significance $\left(\sigma_{\mathbb{E}_{T}}\right)$, is employed. It is given by the likelihood that the measured $\mathbb{E}_{T}$ is consistent with a fluctuation from zero as the result of the detector momentum resolution. The variable is computed on an event-by-event basis and defined as follows: 


$$
\operatorname{METSig}=2 \log \left(\frac{E_{T}}{\sqrt{2} \sigma}\right)
$$

where $\mathbb{E}_{T}$ is the measured missing $E_{T}$ and $\sigma$ is the variance of the probability distribution $p\left(E_{T}\right)$. The $p\left(E_{T}\right)$ and the variance $\sigma$ are computed by the $\sigma_{\mathscr{E}_{T}}$ algorithm [94] from the object momentum resolutions assuming they have a Gaussian shape. The definition of $\sigma_{\mathscr{E}_{T}}$ reflects how many standard deviations the measured $\mathbb{E}_{T}$ is away from zero. Large $\sigma_{\mathbb{E}_{T}}$ values indicate that missing $E_{T}$ is due to the presence of one or more neutrinos in the event, whereas $\sigma_{\mathbb{E}_{T}}$ values closer to zero correspond to $\mathbb{E}_{T}$ from mismeasurement.

Although the $\sigma_{\mathscr{E}_{T}}$ varies in the range from 0 to infinity, for many analyses cuts on $\sigma_{\mathscr{E}_{T}}$ usually lie in interval from 0 to 10 . A cut on $\sigma_{\mathscr{E}_{T}}$ is found to be very useful to reduce $Z$ background for $t \bar{t}$ events. 
(a)

(b)
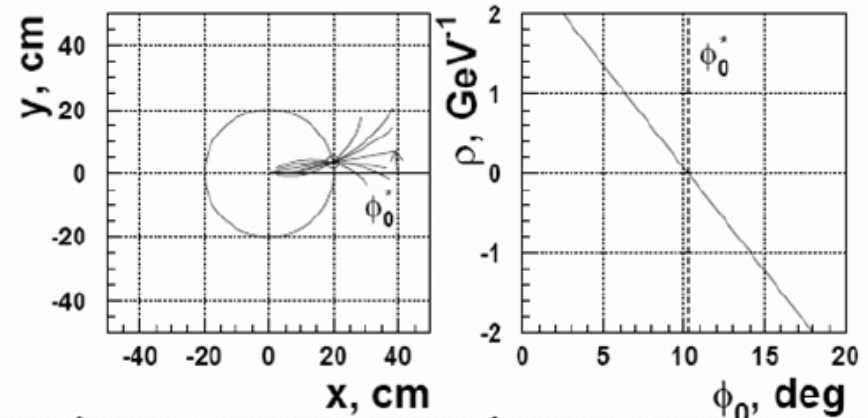

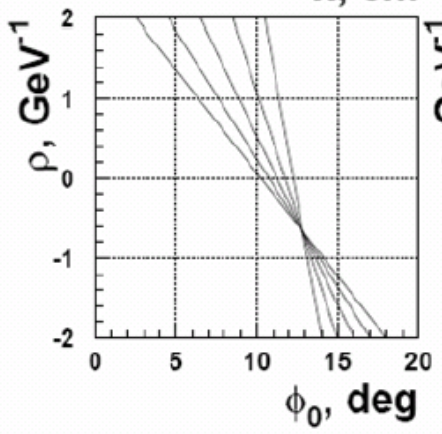

(c)

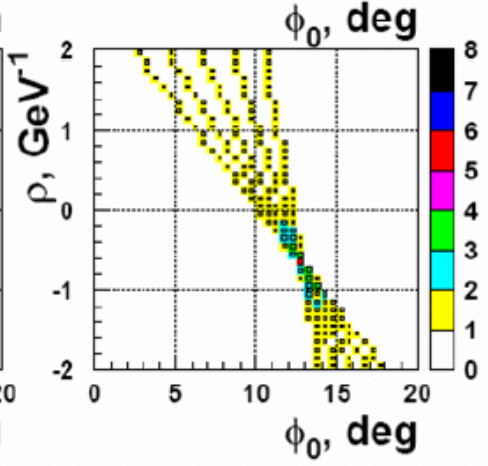

(d)

Figure 5.2. The illustration of the Hough transformation for a single muon of $1.5 \mathrm{GeV}$ leaving 5 hits in the SMT. (a) Circular trajectories passing through the given hit. (b) A line in the $(\rho, \phi)$ space that corresponds to the set of trajectories for the hit. (c) Five hits correspond to the five lines crossing at one point in $(\rho, \phi)$ space. (d) 2D histogram with the peak giving track candidate parameters. 


\section{Chapter 6}

\section{JET ENERGY CALIBRATION}

The energy of a jet calculated by the RunII Cone Algorithm, in principle, does not match to the total energy of all particles the jet encompasses. This is a result of different detector effects and algorithm limitations. For instance, non-liniarities in hadron response and presence of dead material in the calorimeter result in a lower measured jet energy than the total energy of all constituent jet particles. Such an algorithm limitation as the fixed size of a cone does not account for particle emission outside of the cone and therefore also leads to the lower measured energy of a jet. Thus to determine jet energy accurately, jet specific corrections are required.

The Jet Energy Scale (JES) is a general term for the corrections that account for the effects mentioned above. Section 6.1 describes the standard approach used at DØ to correct the jet energies. The standard JES is derived on the $\gamma+$ jet sample while the analysis specific corrections are obtained from $\ell+$ jets channel of $t \bar{t}$ decay. The $\gamma+$ jet sample suffers from large contamination from dijet events. Thus, the purity of $\gamma+$ jet sample is estimated in Section 6.1.2.

The analysis specific corrections, flavor-dependent jet response correction and $\ell+$ jets jet energy scale correction, are described in Section 6.2. It is a first time that these corrections are applied in the $t \bar{t}$ dilepton channel.

\subsection{Standard Jet Energy Scale Correction}

As mentioned above, a parton - gluon or quark - had never been observed in isolation. A schematic view of parton evolution starting from the hard scattering interaction to the 
energy deposition in the calorimeter cells is depicted in Figure 6.1. Due to the nature of the strong interaction, a high-energy parton produced in the collision consequently goes through showering and hadronization. As the result of this, a jet of hadrons is produced. Such a collimated shower of particles before interacting with the detector is often referred as a particle jet. Particle jet is reconstructed with the same algorithm as a jet at the detector level. Since the showering and fragmentation make it very hard to associate the jet energy with an underlying parton energy, the standard JES tries to relate a jet seen in the calorimeter with a particle jet. The ultimate goal of the jet energy scale is to correct the energy of a reconstructed jet back to the particle level jet, but not to the parton level.

The jet energy scale correction procedure is made of a number of sub-corrections. Each correction is derived independently and later applied sequentially with the other corrections.

The following sub-corrections [95] are the main components used for the standard jet energy scale correction procedure:

- Offset correction $E_{\text {offset }}$. The offset correction $E_{\text {offset }}$ is the energy deposited in the calorimeter in the cone of jet that is not associated with any hard scattering processes. The offset energy is the result of the underlying event. The underlying event includes all possible effects in the calorimeter that lead to the energy deposition, except for the inelastic collisions. Such effects, for example, are multiple parton interactions, beam remnant energy deposits, energy from previous collisions.

The offset correction is determined from the measurement of the energy density per tower measured in minimum bias events. In order to take into account a possible dependence on the instantaneous luminosity, the measurement of energy density is performed for different primary vertex multiplicities. 


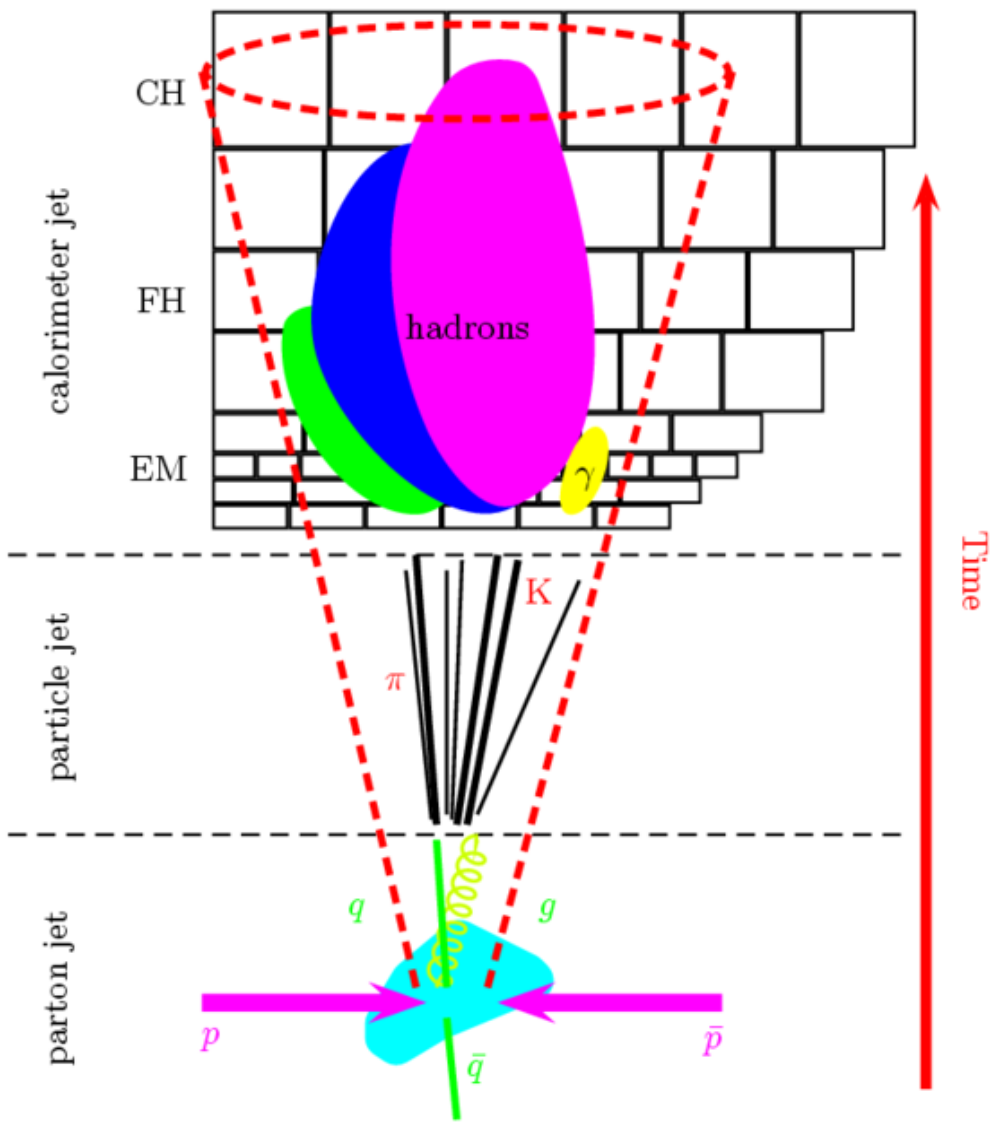

Figure 6.1. Sketch of a parton evolution into a jet in the calorimeter. The original parton is the result of hard scattering $p \bar{p}$ interaction. 
- Relative response correction $F_{\eta}$. The $\mathrm{D} \varnothing$ detector is uniform in the central cryostat (CC), and the end-cap cryostat (EC). Although, it is not uniform in the regions between these components. The relative response correction attempts to make the calorimeter look uniform across wide range in pseudorapidity. In the idealistic situation, after this correction is applied, the calorimeter has a response to a jet versus energy that is independent of $\eta$.

- Absolute response correction $R$. The absolute response is defined as

$$
R=\frac{\sum_{i \in p t c l j e t} E_{i}^{\text {meas }}}{E_{\text {jet }}^{\text {ptcl }}}
$$

where $E_{i}^{\text {meas }}$ is the energy deposited in the calorimeter by the $i$-th particle from a particle jet, $E_{j e t}^{p t c l}$ is the energy of particle jet. After the relative response and the offset corrections have been applied, the absolute response is determined for the jets in the region with $|\eta|<1.0$ (CC) and $1.5<|\eta|<2.5$ (EC).

- Detector showering correction $S$. The showering correction is aimed to correct for the energy losses (gains) out of (in to) the jet cone due to the particle showers leaking in to (out of) the jet cone. The showering correction is defined as

$$
S=\frac{\sum_{i \in p t c l j e t} E_{i}^{\text {meas }} S_{i}+\sum_{i \notin p t c l j e t} E_{i}^{\text {meas }} S_{i}}{\sum_{i \in \text { ptcljet }} E_{i}^{\text {meas }}}
$$

where $S_{i}$ is the energy fraction of the $i$-th particle contained within the calorimeter jet cone.

Since the detector modeling is not perfect, jets in the MC simulation have different response than those in data. Thus, the response correction is determined separately for the MC simulation and the data. The equation used to correct the energy of a reconstructed jet to the particle level is given by

$$
E_{j e t}^{p t c l}=\frac{E_{j e t}^{r e c o}-E_{o f f s e t}}{F_{\eta} \times R \times S}
$$


where $E_{j e t}^{r e c o}$ is the measured jet energy in the calorimeter, and $E_{j e t}^{p t c l}$ is the corrected jet energy to the particle level.

\subsubsection{Missing $E_{T}$ Projection Fraction Method}

The uncertainty from the standard JES correction is dominated by the error on the jet response in the calorimeter. Thus, it is of great importance to have a reliable and robust method for the calorimeter response calculation. At D $\varnothing$, the absolute jet response is measured with the Missing $E_{T}$ Projection Fraction (MPF) method [96] using $p_{T}$ imbalance in events containing a photon with a jet back-to-back $(\gamma+$ jet $)$.

Compton scattering and quark-antiquark annihilation are the main physics processes leading to such direct photon production. The leading order Feynmann diagrams responsible are shown in Figure 6.2
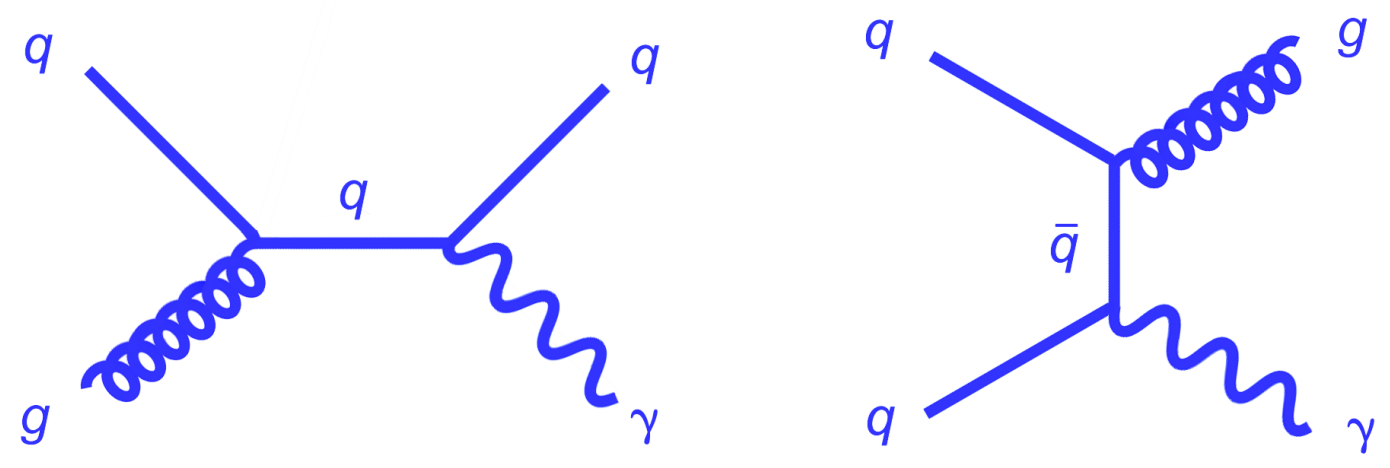

Figure 6.2. The main Feynmann diagrams of the direct photon production in $\gamma+$ jet events.

The MPF method is based on the momentum conservation law. Although it is applied to events from higher orders, it is instructive to consider further the LO configuration. 
The total transverse momentum of $p \bar{p}$ before the collision is zero; thus in the $\gamma+$ jet finals state events the sum of the photon transverse momentum $\left(\vec{p}_{T}^{\gamma}\right)$ and the particle jet transverse momentum $\left(\vec{p}_{T}^{\text {ptcljet }}\right)$ is also zero

$$
\vec{p}_{T}^{\gamma}+\vec{p}_{T}^{\text {ptcljet }}=0
$$

In Equation 6.4, the effect of hadronization is neglected and it is assumed that the transverse momentum of the underlying parton is equal the transverse momentum of the resulting particle jet, $\vec{p}_{T}^{\text {parton }}=\vec{p}_{T}^{\text {ptcljet }}$. The photon is often referred as a tag, while the jet is called a probe.

Due to the different hadronic and electromagnetic responses, the actual energy measurement in the event will manifest missing $E_{T}$. So the momentum conservation for the identified objects in the calorimeter is given by

$$
R_{e m} \vec{p}_{T}^{\gamma}+R_{j e t} \vec{p}_{T}^{p t c l j e t}+\overrightarrow{\not ̈}_{T}^{\text {meas }}=0
$$

where $R_{e m}$ is the electromagnetic response, and $R_{\text {jet }}$ is the response of the particle jet.

Since the $R_{e m}$ is a known parameter, then the corrected missing energy can be defined as follows

$$
\overrightarrow{\not E}_{T}^{c o r r}=\vec{\not}_{T}^{\text {meas }}-\left(1-R_{e m}\right) \vec{p}_{T}^{\gamma}
$$

Using the relationship $\vec{p}_{T}^{\text {ptcljet }}=-\vec{p}_{T}^{\gamma}$ from Equation 6.4 and the corrected missing energy, the Equation 6.5 can be written as

$$
\vec{p}_{T}^{\gamma}-R_{j e t} \vec{p}_{T}^{\gamma}=-\vec{\not}_{T}^{c o r r}
$$

By projecting the missing energy vector along the direction of the photon, the expression for the particle jet response can be written in the following form 


$$
-\hat{n}_{T}^{\gamma} \cdot \vec{H}_{T}^{c o r r}=\vec{n}_{T}^{\gamma} \cdot\left(\vec{p}_{T}^{\gamma}-R_{j e t} \vec{p}_{T}^{\gamma}\right)=p_{T}^{\gamma}-R_{j e t} p_{T}^{\gamma}
$$

Rearranging terms gives,

$$
R_{j e t}=1+\frac{\vec{E}_{T}^{c o r r} \cdot \hat{n}_{T}^{\gamma}}{p_{T}^{\gamma}}
$$

One of the benefits of using the $\vec{E}_{T}$ is that it provides an independent assessment of the offset and showering effects. The offset is expected to be equal in all directions and therefore cancel out when computing $\vec{E}_{T}$. Additionally, the MPF method does not depend on the algorithm that is chosen to reconstruct a jet, because the $\vec{B}_{T}$ is ignorant of the jet cone boundary.

To avoid possible bias in response calculation due to the poor resolution of the measured jet energy, a special jet Energy Estimator is introduced [97]:

$$
E^{\prime}=E_{T \gamma} \cosh \eta_{j e t}
$$

The estimator $E^{\prime}$ is defined through the well-measured quantities: jet direction and photon energy. It is therefore independent of a jet algorithm chosen for jet reconstruction. By measuring the response versus $E^{\prime}$, the jet resolution bias is removed. Although the new bias from a photon is now introduced, the effect of it is much smaller than that from the jet resolution.

The Energy Estimator is strongly correlated with the measured jet energy. For a leading order event, taking into account

$$
\cosh \eta_{j e t}=\frac{1}{\sin \theta_{j e t}}
$$

and neglecting the photon resolution effect, $E_{T \gamma} \simeq E_{T \text { ptcljet }}$, the Energy Estimator

$$
E^{\prime}=E_{\text {ptcljet }}
$$




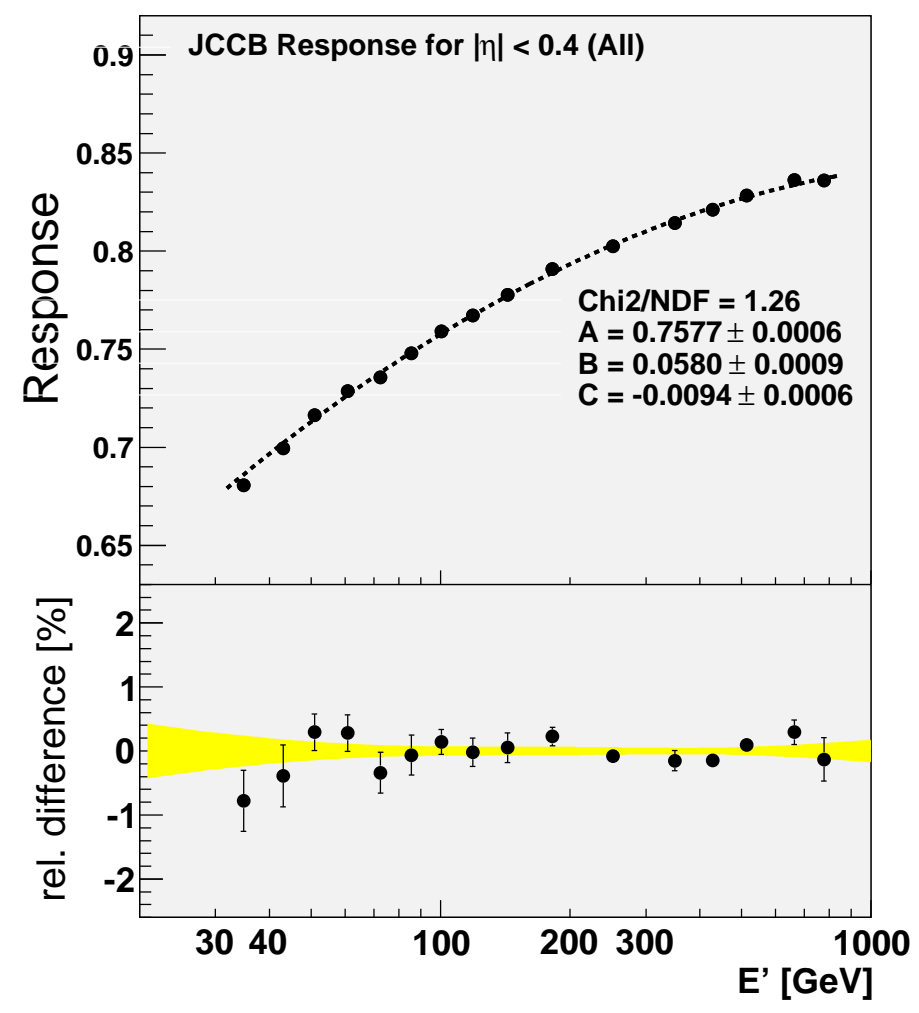

Figure 6.3. The jet response calculated for $J C C B$ jets in RunIIa MC simulation. The JCCB jet is a jet with the cone size $\Delta R=0.5$. The jets with the cone size $\Delta R=0.7$ is called JCCA. 
The jet response dependence on energy estimated for RunIIa MC simulation is shown in Figure 6.3. Based on the electromagnetic fraction $f_{E M}$, parametrization for a single particle in [98], after some manipulation the jet response parametrization, can be written in the following form:

$$
R_{j e t}\left(E^{\prime}\right)=p_{0}+p_{1} \ln \frac{E^{\prime}}{E_{0}}-p_{2} \ln ^{2} \frac{E^{\prime}}{E_{0}}
$$

where $p_{0}, p_{1}$, and $p_{2}$ are independent of energy free parameters; the $E_{0}$ is an arbitrary energy constant. The jet response measured in the data shows very good agreement with the parametrization given in the Equation 6.11.

\subsubsection{Sample Purity Estimation}

As it is described above, the MPF method calculates the absolute jet response from $\gamma+$ jet events. In practice, however, the selected $\gamma+$ jet data sample suffers from contamination of events with two back-to-back jets in the final state. The two jet final state is also known as a dijet event. If one of the jets in a dijet event contains a leading $\pi^{0}$ (or $\eta, \omega, K_{0}^{s}$ ) hadron decaying into two photons, such jet can be potentially misidentified as a photon. The probability of misidentification depends on the photon identification criteria and is typically very small.

Nevertheless, the dijet background contamination still remains a sizeable one. The substantial dijet contamination is mainly the result of much higher dijet event production rate than that for direct $\gamma+$ jet production. The dijet cross section is about three orders of magnitude larger than the cross section with $\gamma+$ jet final state. To take into account the dijet background effect in the absolute response calculation, the sample purity is introduced:

$$
\rho=\frac{S}{S+B}
$$


where $\rho$ is the sample purity, $\mathrm{S}$ is the number of the signal $(\gamma+$ jet $)$ events, and B is the number of background dijet events.

I calculated the purity for medium and tight photon definitions. I also corrected response for the presence of the dijet background. However, because the medium photon criterion is deprecated for most of the analyses at $\mathrm{D} \varnothing$, the only tight photon purity is implemented in the $\mathrm{D} \varnothing$ software framework. The medium photon purity is only needed for tight photon purity estimation. The purities calculated in data and MC simulation are found to be in good agreement.

\subsubsection{Hollow Cone Template Method}

Unlike the MC simulation where the sample purity is calculated by counting the number of events passing the selection criteria, the purity estimation for data is not as straight-forward. I used a special technique called the Hollow Cone Template Method that has been developed to determine the purity.

The method employs a particular variable to discriminate a photon against a jet. The hollow cone track variable ( $\mathrm{HC} 07)$ is defined as the total scalar transverse momenta of all tracks in the hollow cone of $0.05<\Delta R<0.7$ around the photon candidate. The method produces the HC07 distributions for the MC simulation of $\gamma+$ jet and dijet events, and fits those to the distribution in the data. An example of the hollow cone variable distribution for MC signal, MC background, and data is given in Figure 6.4.

In the context of the method, the $\mathrm{HC} 07$ distribution in $\gamma+$ jet and dijet MC samples are referred as the templates. The actual fitting is done with the help of TFractionFitter class from ROOT on the normalized MC templates. Using finite MC samples, TFractionFitter employs special technique [99] to fit a sum of models to the data. The purity is concluded from the fit and indicates what fraction of the events in data is contributed from $\gamma+$ jet production. 


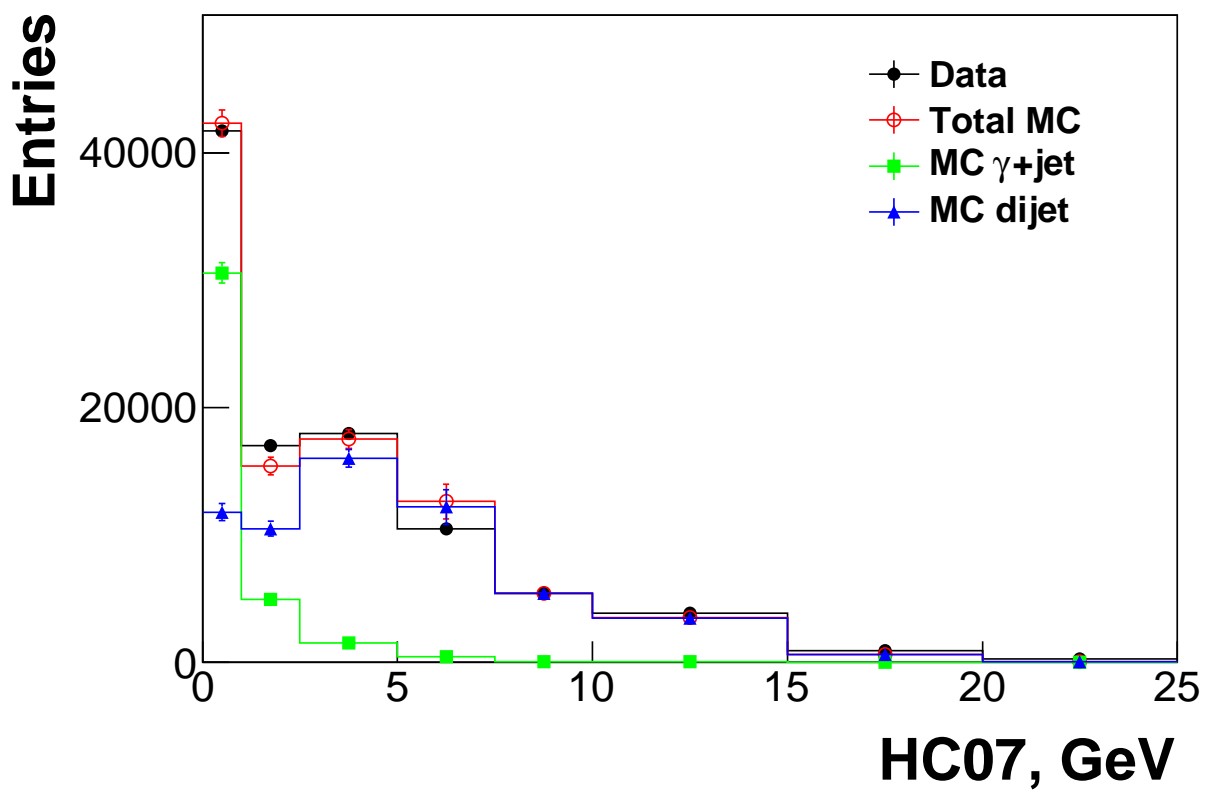

Figure 6.4. The hollow cone track variable distributions in the region with $0.0<\eta_{\text {jet }}<$ 0.4 and $40<E^{\prime}<60 \mathrm{GeV}$. The distribution for the data is shown by the filled circles, while MC simulations are: signal template (squares), background template (triangles), and $\mathrm{MC}$ fit to the data (empty circles). 
The purity is derived for different regions in $\eta_{j e t}$ as a function of $E^{\prime}$, and fitted using the parametrization given in Equation (6.13).

$$
\rho\left(E^{\prime}\right)=\frac{1}{1+p_{0} E^{\prime p_{1}}}
$$

The free parameters $p_{0}$ and $p_{1}$ are independent of energy and determined from the fit. An example of the purity fitted with function (6.13) is shown in Figure 6.5

For the photon candidate with tight quality requirements, the template method cannot be applied directly. The tight photon definition requires $H C 07<1.0 \mathrm{GeV}$, thus making it impossible to produce hollow cone distribution in this case. Instead, purity result $P_{\text {tight }}$ for tight photon is obtained from purity $P_{\text {med }}$ calculated for the medium photon. The tight photon purity $P_{\text {tight }}$ is given by

$$
P_{\text {tight }}=\frac{P_{\text {med }} \cdot f_{\text {med }}^{\gamma+j e t}}{P_{\text {med }} \cdot f_{m e d}^{\gamma+j e t}+\left(1-P_{\text {med }}\right) \cdot f_{m e d}^{\text {dijet }}}
$$

where $f_{\text {med }}^{\gamma+j e t}$ and $f_{\text {med }}^{\text {dijet }}$ is fraction of events passing $H C 07<1.0 \mathrm{GeV}$ for medium quality photon in $\mathrm{MC}$ simulated $\gamma+$ jet and dijet samples. The $P_{\text {med }}$ used in Equation (6.14) is derived with the $\mathrm{HC} 07$ template method while $f_{\text {med }}^{\gamma+j e t}$ and $f_{\text {med }}^{\text {dijet }}$ are estimated from MC simulation. Equation (6.14) becomes the standard definition of the purity for tight photon, $P_{\text {tight }}=\frac{S_{\text {tight }}}{S_{\text {tight }}+B_{\text {tight }}}$, given that $f_{\text {med }}^{\gamma+j e t} \equiv \frac{S_{\text {tight }}}{S_{\text {med }}}, f_{\text {med }}^{\text {dijet }} \equiv \frac{B_{\text {tight }}}{B_{\text {med }}}$ and $P_{\text {med }}=$ $\frac{S_{m e d}}{S_{m e d}+B_{m e d}}$

\subsection{Analysis-Specific Jet Energy Scale Corrections}

With more data collected, the statistical error of a measurement becomes small or even negligible. As opposed to that, an increase in data does not necessarily affect a systematic uncertainty and thus can leave it unchanged. For the analyses with jets in the final state, such as the measurement of the top quark mass, QCD precision tests 

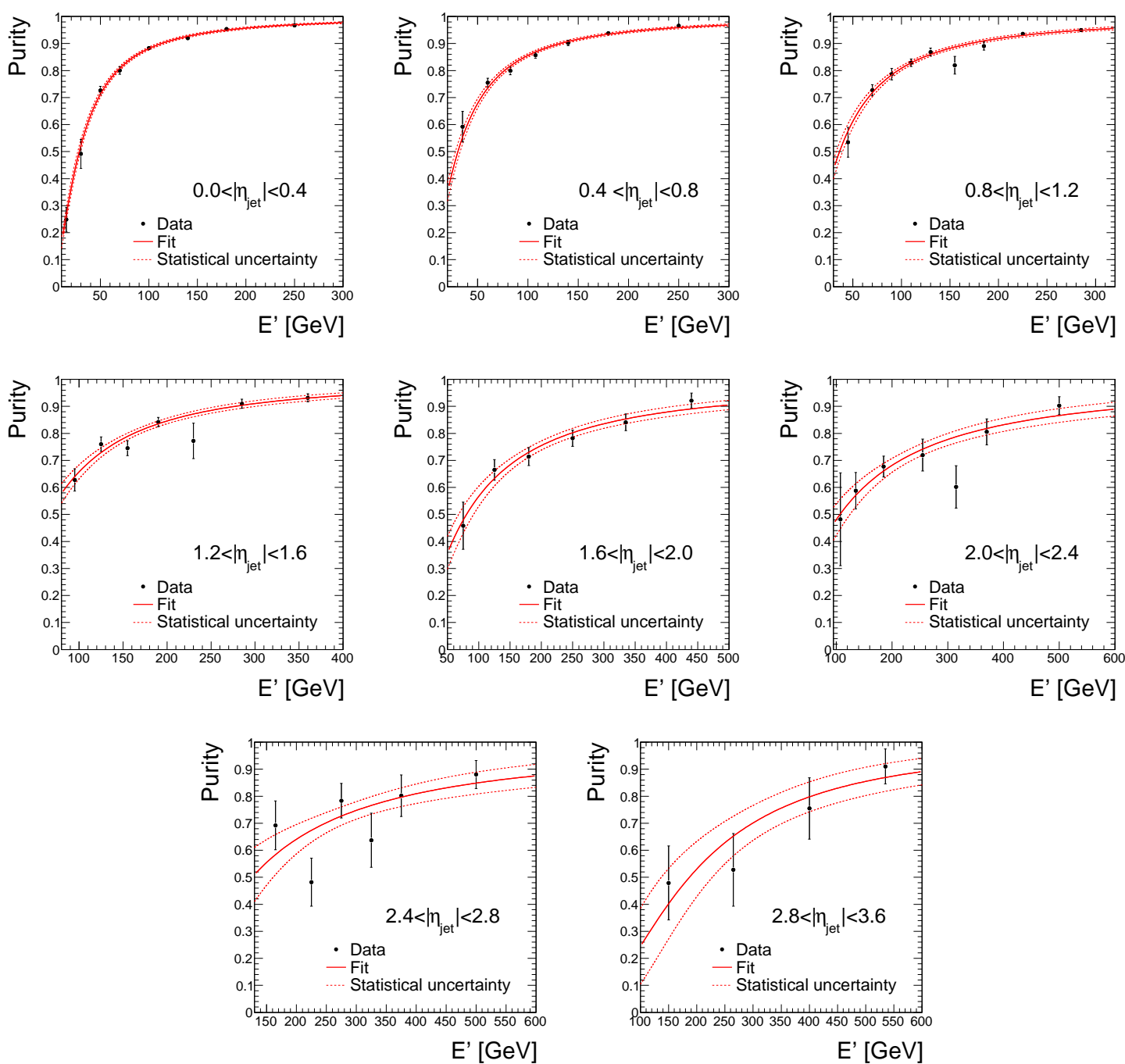

Figure 6.5. Sample purity calculated with the hollow cone method in the different $\eta_{j e t}$ regions. The purity is shown in the black circles with corresponding error bars reflecting the uncertainty. The parametrization fit is the solid red line. Dashed red bands indicate the statistical uncertainty on the fit. 
and others, the error due to the Jet Energy Scale is the major systematic uncertainty. As a result of that, the total uncertainty is largely dominated by the JES systematic uncertainty.

In order to reduce the JES systematic and lower the total uncertainty for the analysis presented in this thesis, I have applied additional jet response corrections. A flavor dependent correction applies to $\mathrm{MC}$ simulation and accounts for a difference in response between $b$-jets and light quark jets. The global scale discrepancy between jets in the simulated events and the events from data is accounted using an average response correction obtained from the top quark mass measurement in the $\ell+$ jets channel.

\subsubsection{Flavor-Dependent Jet Response Correction}

The energy of a jet measured in the calorimeter is corrected to the particle level by the standard Jet Energy Scale procedure described in the Section 6.1. The standard JES is derived from $\gamma+$ jet events and depends on the jet kinematics, i.e. jet pseudorapidity and transverse momentum. This, however, does not account for the fact that jets can be initiated from partons of different flavor. Different underlying partons give the jets differing in particle composition and kinematic characteristics. As a result, the electromagnetic fraction of the energy in the calorimeter, for instance, is different for $b$ and light jets. More importantly, particle multiplicity for $b$ and light jets is also very different [100].

The type, energy spectra and angular distribution of particles constituting a jet is a result of the topological configuration of the event. The number of particles in a jet is also affected by event topology. Initiated from different flavors of parent partons, jets have different particle distributions and ideally should therefore have different response correction. An example of particle jet multiplicity for gluon and quark jet is given in Figure 6.6 [101]. 


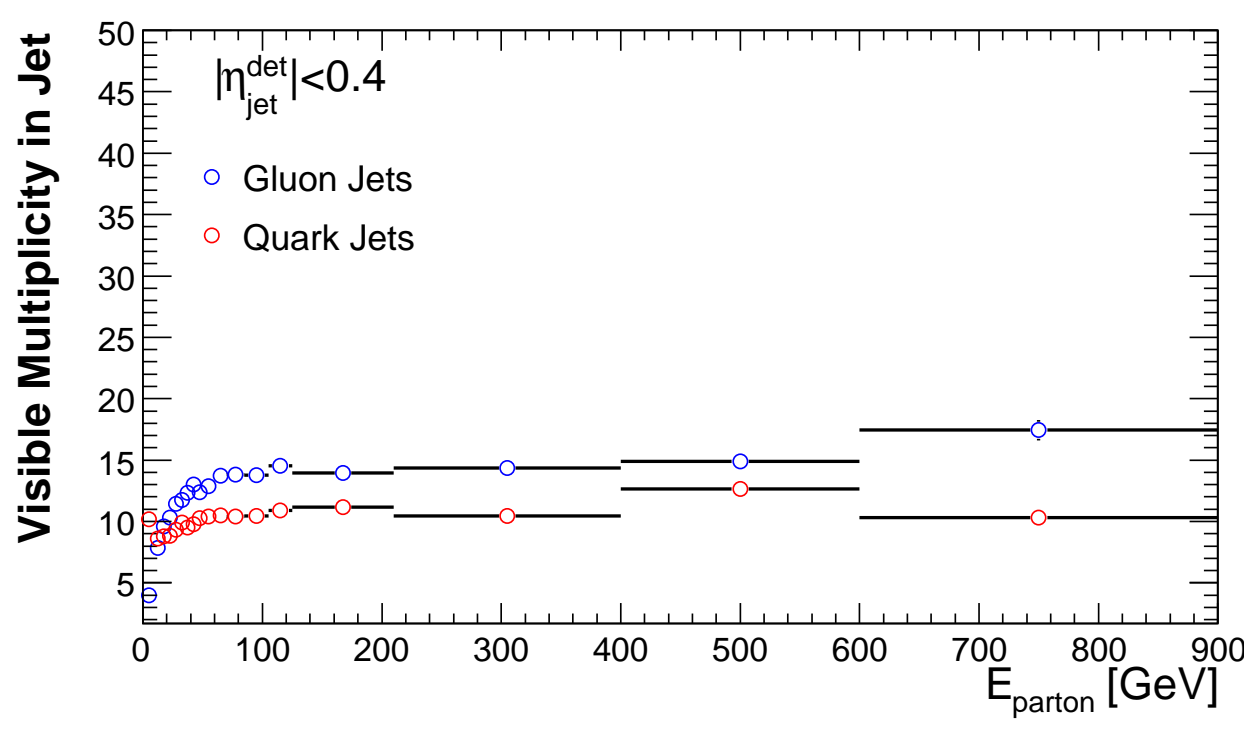

Figure 6.6. Number of particles in a jet initiated from gluon (blue) and quark (red). 
If not properly corrected for, a different particle distribution of jets in MC simulation vs. data can result in a systematic shift in the measurement of the top quark mass. To make the calorimeter jet response in the simulation agree with that in data, the flavordependent response correction [102] is applied to the MC.

Each MC simulated jet in the calorimeter is spatially matched to a particle level jet. The correction factor for the jet response is calculated as follows

$$
F=\frac{\sum_{i} E_{i} \cdot R_{i}^{\text {Data }}}{\sum_{i} E_{i} \cdot R_{i}^{M C}}
$$

where the sums run over all particles in the MC particle jet; $E_{i}$ is the true energy of the $i$-th particle; $R_{i}^{M C}$ and $R_{i}^{D a t a}$ are the single-particle responses of the $i$-th particle for the simulation and data correspondingly. The correction factor $F$ is applied to the jet energy after the offset correction $E_{\text {offset }}$ is subtracted

$$
\left(E_{j e t}^{r e c o}-E_{\text {offset }}\right)^{\text {corr }}=\left(E_{j e t}^{r e c o}-E_{\text {offset }}\right) \cdot F
$$

The other standard JES corrections, i.e. $R, F_{\eta}$ and $S$ are applied to the $\left(E_{j e t}^{r e c o}-\right.$ $\left.E_{\text {offset }}\right)^{\text {corr }}$ in the regular way according to Equation (6.3):

$$
E_{\text {jet }}^{p t c l, c o r r}=\frac{\left(E_{j e t}^{r e c o}-E_{o f f s e t}\right)^{c o r r}}{F_{\eta} \times R \times S}
$$

Single particle responses used in Equation (6.16) are derived separately for data and MC simulation. All stable particles except the neutrinos - $\gamma, e^{ \pm}, \mu^{ \pm}, \pi^{ \pm}, K^{ \pm}, K_{0}^{S}, K_{0}^{L}$, $p^{ \pm}, n, \Lambda, \Sigma$ 's, $\Xi$ 's are included into particle jet reconstruction.

In practice, however, Equation (6.17) is not used to correct the energy as it would require to re-derive the standard JES correction from the sample corrected for the MC to data difference. Instead, the ratio between $F$ for different flavors of jets and the averaged $F$ for the jets in the $\gamma+$ jet sample is used 


$$
\mathcal{D}_{\text {corr }}^{\beta}=\frac{F^{\beta}}{\left\langle F^{\gamma+j e t}\right\rangle}
$$

where $\beta$ labels a jet initiated by a light quark, gluon, or $b$ quark. Defining the correction this way allows to correct the average jet response for the $\mathrm{MC}$ to data difference for different flavors of jets.

\subsection{2. $\ell+$ jets Jet Energy Scale Correction}

To further calibrate the energy of jets in data, the analysis of this thesis uses an additional jet energy scale factor, referred to below as $k_{J E S}$. The correction factor is calculated in the $\ell+$ jets channel of the $t \bar{t}$ decay, and the most recent such analysis by $\mathrm{D} \varnothing$ that derives $k_{J E S}$ is the measurement of the top quark mass with the Matrix Element method [103].

In the $\ell+$ jets channel of $t \bar{t}$ events, the scale factor $k_{J E S}$ is applied to the energies of all jets. Subsequently, a likelihood as a function of $m_{t}$ and $k_{J E S}$ is constructed. The top quark mass and jet energy scale are then obtained by maximizing the likelihood. Since one of the $W$ bosons decays hadronically, $W \rightarrow q q^{\prime}$, the invariant mass of the $W$ boson is used to constraint the resulting pair of jets $W$ bosons from decays. According to the results from Ref. [103], the scale factor is measured to be $k_{J E S}=1.013 \pm 0.008$ (stat). The resulting two-dimensional likelihood contours after all calibration are shown in Figure 6.7 taken from Ref. [103].

The method employed in Ref. [103] to derive $k_{J E S}$ is channel-specific and care must be taken when directly applying it to $t \bar{t}$ dilepton events. The requires that the difference in event topologies between the two channels are properly accounted.

To calibrate energy of jets in data, all jets in an event have to be divided by $k_{J E S}$.

The mean value of $k_{J E S}$ is used to correct the jet energies, and the effect from its statistical uncertainty is calculated as a corresponding systematic uncertainty in Chapter 


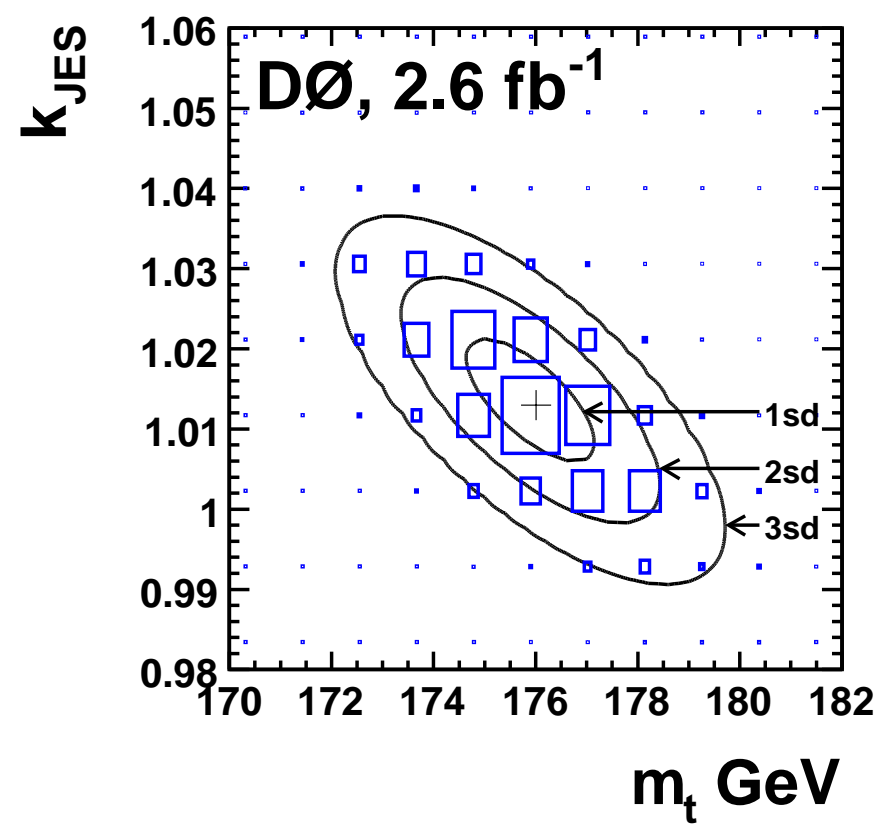

Figure 6.7. Fitted Gaussian contours of equal probability for the two-dimensional likelihoods as a function of $m_{t}$ and $k_{J E S}$. The contours correspond to a 1- $\sigma, 2-\sigma$, and 3- $\sigma$ statistical uncertainty on $m_{t}$. 
8. The application of the $\ell+$ jets jet calibration in the dilepton channel allows us to replace the standard JES systematic uncertainty by the statistical uncertainty on $k_{J E S}$ and uncertainties of transferring the calibration to the different dilepton event topology. The combined uncertainty due to effects of carrying over the $\ell+$ jets calibration is substantially smaller than that of the standard jet energy scale correction.

\subsubsection{Adopting the $\ell+j e t s$ Jet Energy Scale}

The event topology in $\ell+$ jets differs from that in dilepton channel primarily due to the higher jet multiplicity. In a high-jet-multiplicity environment, the hadrons can be often misrecognized by the jet reconstructing algorithm as belonging to an incorrect jet. The different color flow scenarios in $\ell+$ jets and dilepton channels have an impact on reconstructed jet energy. In the $\ell+$ jets channel, the hadronic decay of a color singlet $W$ boson produces two quarks forming a color dipole. The showering process of the quarks from the color dipole produces more radiation in the region between the quarks. Non-uniform dipole radiation complicates jet reconstruction affecting jet energies.

To adopt the $\ell+$ jets energy correction in dilepton events, I examined the effect of a possible difference between the two channels in the jet energy scales for the $b$-quark. The difference can be evaluated using the following response double ratio

$$
\mathcal{R}_{2 \ell}^{b}\left(p_{T}^{b}\right)=\frac{R_{\text {data }}^{2 \ell}\left(p_{T}^{b}\right) / R_{M C}^{2 \ell}\left(p_{T}^{b}\right)}{R_{\text {data }}^{\ell+j e t s}\left(p_{T}^{b}\right) / R_{M C}^{\ell+j e t s}\left(p_{T}^{b}\right)}
$$

where $R_{\text {data }}^{2 \ell}$ and $R_{M C}^{2 \ell}$ are the $b$-jet energy response for $\mathrm{MC}$ and data sample in the dilepton channel, $R_{\text {data }}^{\ell+j e t s}$ and $R_{M C}^{\ell+j e t s}$ are the respective responses for $\ell+$ jets channel. The jet energy scale responses $R_{\text {data }}^{2 \ell}, R_{M C}^{2 \ell}, R_{\text {data }}^{\ell+j e t s}$ and $R_{M C}^{\ell+j e t s}$ are derived using known responses for single particles in data and MC. The double ratio as a function of $b$-jet transverse momentum is shown in Figure 6.8. 


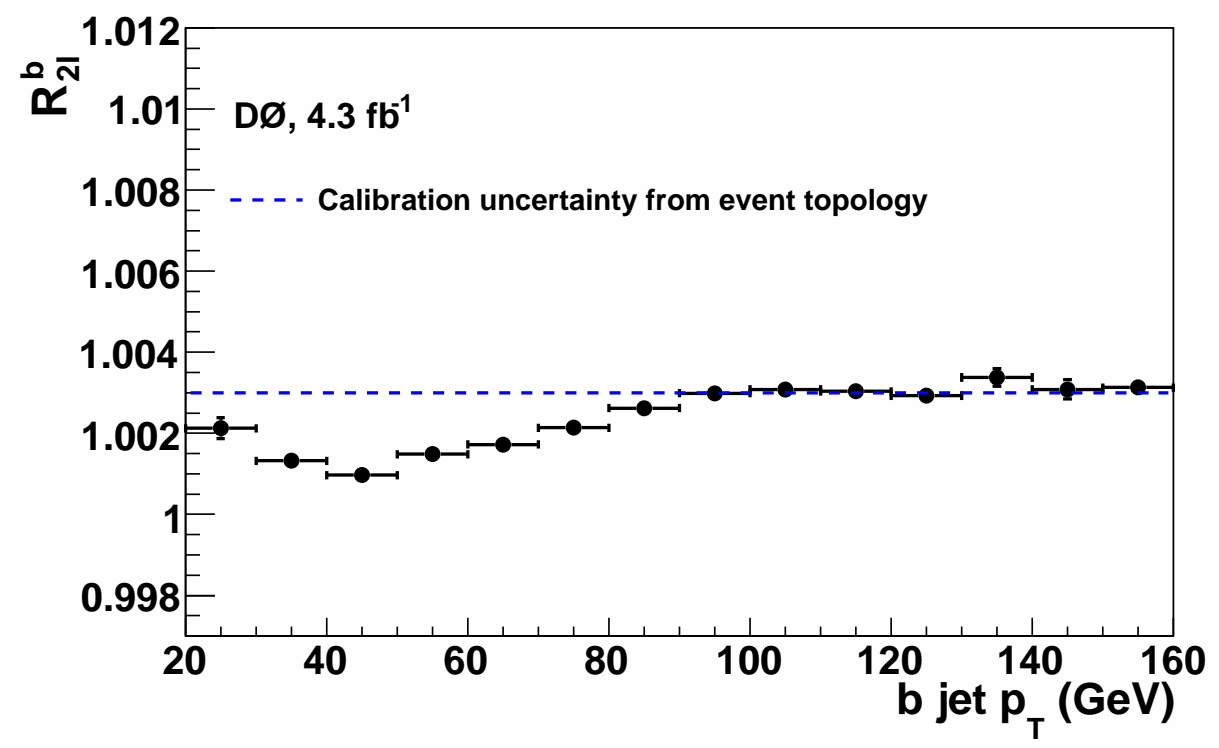

Figure 6.8. The double ratio, $R_{2 \ell}^{b}$, is plotted vs $b$-jet transverse momentum. The value of the double ratio in each bin is shown in black circles with corresponding error bars reflecting the uncertainty. Dashed blue line is the asymptotic value of the double ratio. 
As can be seen in Figure 6.8, the double ratio varies in the range from 1.001 to 1.003 depending of the $b$-jet transverse momentum value. If the event topologies for both channels were equal, the double ratio would equal unity. The maximum difference of $0.3 \%$ from unity shows that $\mathcal{R}_{2 \ell}^{b}\left(p_{T}^{b}\right)$ is very close to one and therefore the channels have almost identical jet energy scales of the $b$-quark jets.

The small variation of the double ratio from one can be a result of different jet particle multiplicity. Table 6.1 gives the average number of particles in a jet for $\ell+$ jets and dilepton channels. The particle multiplicity of jets in $\ell+$ jets MC simulated sample is a few percent higher than that in the dilepton sample. This is enough to produce an observed shift in the double ratio up to $0.3 \%$.

The $\ell+$ jets jet energy scale is applied to dilepton events in the analysis of this thesis, and the difference in $b$-jet responses between the channels is taken into the account as systematic effect. The difference between the asymptotic value of $\mathcal{R}_{2 \ell}^{b}$ and unity is reflected as part of the systematic uncertainty on adopting the $\ell+$ jets scale. The details on calculation of the systematic effect due to carrying over $k_{J E S}$ to dilepton sample can be found in Section 8.3.2.1.

Table 6.1. Average number of particles in a jet for $\ell+$ jets and dilepton channels.

\begin{tabular}{l|ccc}
\hline \hline \multirow{2}{*}{ Jet $p_{t}$ range } & \multicolumn{3}{|c}{ Average number of particles in a jet } \\
\cline { 2 - 4 } & $b$-jet in $\ell+$ jets & $b$-jet in dilepton & light jet in $\ell+$ jets \\
\hline$[60,70] \mathrm{GeV}$ & 23.55 & 22.96 & 13.56 \\
all available & 21.90 & 21.13 & 11.98 \\
\hline \hline
\end{tabular}




\section{Chapter 7}

\section{THE NEUTRINO WEIGHTING METHOD}

The dilepton final state of $t \bar{t}$ decay consists of two charged leptons, two $b$-jets and two neutrinos. Due to ambiguity in the jet permutations and the presence of neutrinos

in the final state, the event topology does not provide sufficient information to directly reconstruct masses of top and antitop quarks from the measured kinematics. To measure the top quark mass from the available information, a special template based method has been developed and successfully used at D $\varnothing$ and CDF.

The Neutrino Weighting method ( $\nu$ WT) [104], [105], [106] is used to extract the top quark mass and estimate the statistical uncertainty of the measurement in events with two leptons in the final state. The following sections describe kinematics in dilepton events, basics of the the Neutrino Weighting method, maximum likelihood formalism in top quark mass reconstruction, and the pseudoexperiment testing approach to validate and calibrate the method.

\subsection{Kinematic Reconstruction}

There are six particles produced in dilepton final state of $t \bar{t}$ decay: the two charged leptons are either electrons or muons of large transverse momentum; two $b$-jets; and two neutrinos. Each of six final-state particles in the decay is described by an energymomentum four-vector. Therefore, a full description of an event requires $6 \times 4=24$ kinematic parameters. An example of $t \bar{t}$ dilepton decay into $e \mu$ final state is shown in Figure 7.1. 


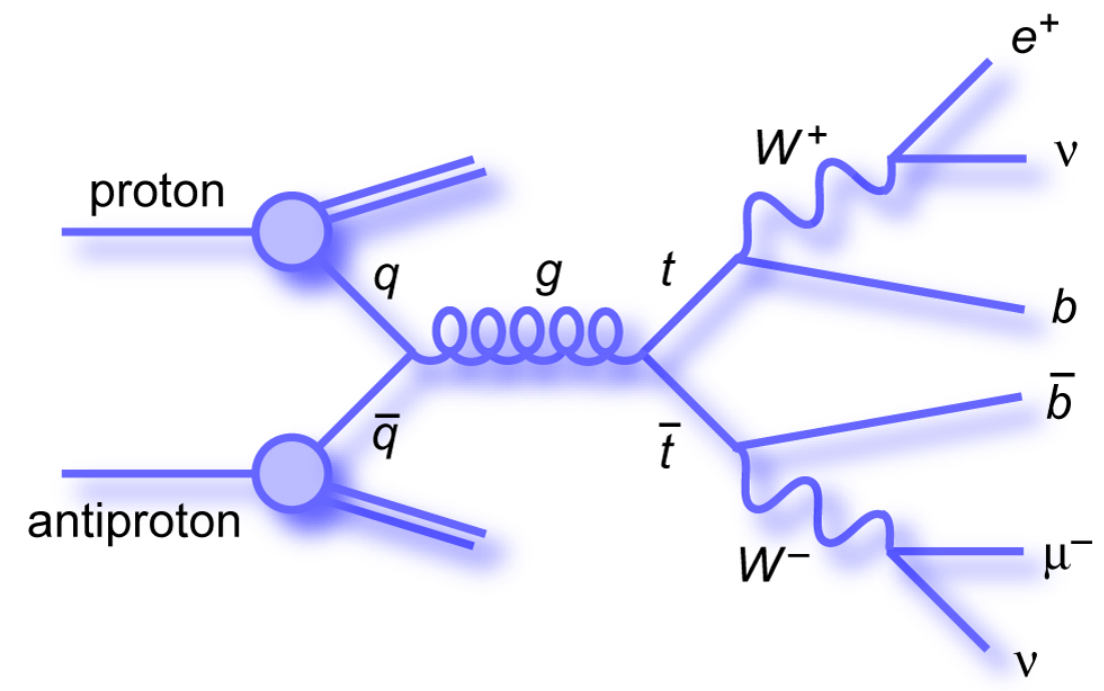

Figure 7.1. Schematic representation of $e \mu$ dilepton decay mode in $t \bar{t}$ event produced in hard-scattering interaction of quark-antiquark pair.

The four-momentum of the leptons and the jets are measured directly in the detector reducing the number of independent parameters to eight $-\mathrm{M}=(24-4 \times 4)=8$. The two jets are assigned to $b$ and $\bar{b}$ quarks from $t$ and $\bar{t}$ decays. The validity of this is verified in the pseudoexperiment testing. The masses of all decay particles are considered to be known. For instance, two neutrinos from each of the $W$ 's are considered massless, $m_{\nu_{1}}=m_{\nu_{2}}=0$. This allows for an additional two constraints, Equation 7.1, and leaves six undetermined parameters, $M=6$. The measurement of $E_{T}^{x}$ and $E_{T}^{y}$ brings an additional two constraints, Equation 7.2, and leaves the number of free parameters $M=4$.

To further reduce the number of degrees of freedom, there are three kinematic constraints that can be constructed. Two constraints can be obtained from the invariant mass of each lepton-neutrino pair. Produced in the decay of the $W$ boson, each leptonneutrino pair is required to have invariant mass equal mass to the mass of $W$ boson, 
$M_{W}$, Equation 7.3 and 7.4. The assumption that masses of the top and the antitop quark are equal, $m_{t}=m_{\bar{t}}$, provides an additional third constraint that can be written in terms of kinematic variables, Equation 7.5. Using the last three constraints reduces the number of unconstrained parameters to $\mathrm{M}=1$.

The system of the equations therefore can be written as follows

$$
\begin{gathered}
E_{\nu_{1}}=\left|\vec{p}_{\nu_{1}}\right|, \quad E_{\nu_{2}}=\left|\vec{p}_{\nu_{2}}\right| \\
E_{T}^{x}=p_{\nu_{1}}^{x}+p_{\nu_{2}}^{x}, \quad E_{T}^{y}=p_{\nu_{1}}^{y}+p_{\nu_{2}}^{y} \\
M_{W_{1}}^{2}=\left(E_{\ell_{1}}+E_{\nu_{1}}\right)^{2}-\left(\vec{p}_{\ell_{1}}+\vec{p}_{\nu_{1}}\right)^{2} \\
M_{W_{2}}^{2}=\left(E_{\ell_{2}}+E_{\nu_{2}}\right)^{2}-\left(\vec{p}_{\ell_{2}}+\vec{p}_{\nu_{2}}\right)^{2} \\
\left(E_{\ell_{1}}+E_{\nu_{1}}+E_{b_{1}}\right)^{2}-\left(\vec{p}_{\ell_{1}}+\vec{p}_{\nu_{1}}+\vec{p}_{b_{1}}\right)^{2}=\left(E_{\ell_{2}}+E_{\nu_{2}}+E_{b_{2}}\right)^{2}-\left(\vec{p}_{\ell_{2}}+\vec{p}_{\nu_{2}}+\vec{p}_{b_{2}}\right)^{2}
\end{gathered}
$$

where $M_{W_{i}}$ is the invariant mass of the $i$-th $W$ boson; $\left(E_{\ell_{i}}, \vec{p}_{\ell_{i}}\right)$, and $\left(E_{\nu_{i}}, \vec{p}_{\nu_{i}}\right)$ are the components of the energy-momentum four-vector of a lepton and neutrino coming from the $i$-th $W$ boson decay; $i$ runs over two $W$ 's produced in the decay of the top and antitop quarks.

The system of equations (7.1) - (7.5) is underconstrained by one degree of freedom, and therefore cannot be solved without additional information provided. The Neutrino Weighting method supplies an additional constraint (cf. Ref. [109]), and estimates the relative weight of how consistent the resulting kinematic solution is with what is observed in the detector.

\subsection{Neutrino Weighting}

To kinematically reconstruct dilepton $t \bar{t}$ event, or equivalently find a solution for the system of equations (7.1) - (7.5), the Neutrino Weighting method assumes the value for the top quark mass. The top quark mass assumption allows us to determine the solution 
for the system of equations, and find the momentum of each neutrino through a second order algebraic equation. This of course does not imply that the solution can be found for any top quark mass assumption. Clearly, some mass assumptions may not have a solution at all.

Given the expected $t \bar{t}$ decay, the goal of the Neutrino Weighting is to indicate what is the most preferred top quark mass. Assuming the value of the top quark mass, $m_{t}$, the $\nu$ WT method can calculate a weight, $W\left(\{o\} \mid m_{t}\right)$, for every event in data or Monte Carlo, where $\{o\}$ is the set of measured 14 kinematic parameters in the event. The weight represents a measure of the probability for an observed final state to originate from $t \bar{t}$ pair of a given mass, and is proportional to the corresponding probability

$$
W\left(\{o\} \mid m_{t}\right) \propto P\left(\{o\} \mid m_{t}\right)
$$

The $P\left(\{o\} \mid m_{t}\right)$ is the probability to observe final state $\{o\}$ given the top quark mass, $m_{t}$. Similarly, $P\left(m_{t} \mid\{o\}\right)$ is the probability to measure the top quark mass equal $m_{t}$ given that the $t \bar{t}$ pair decayed to the observed final state $\{o\}$. Both probabilities are related through Bayes' theorem

$$
P\left(m_{t} \mid\{o\}\right)=\frac{P\left(\{o\} \mid m_{t}\right) P\left(m_{t}\right)}{\int_{0}^{\infty} P\left(\{o\} \mid m_{t}^{\prime}\right) P\left(m_{t}^{\prime}\right) \mathrm{d} m_{t}^{\prime}}
$$

The denominator in Equation (7.7) acts as a normalization factor that ensures $P\left(m_{t} \mid\{o\}\right)$ satisfies Kolmogorov axioms [108] for the probability definition. The $P\left(m_{t}\right)$ is the Bayesian prior probability that reflects the degree of belief the top quark has a certain mass before carrying out an actual experiment.

Assuming no prior knowledge about the top quark having a certain mass, or equivalently that any top quark mass is equally possible, the $P\left(m_{t}\right)$ is taken to be a constant. The relationship between $P\left(m_{t} \mid\{o\}\right)$ and $P\left(\{o\} \mid m_{t}\right)$ can then be re-written as 


$$
P\left(m_{t} \mid\{o\}\right) \propto P\left(\{o\} \mid m_{t}\right)
$$

Taking into the account Equation (7.6) that relates the probability to the weight, the relationship between the weights can be obtained from Equation (7.8)

$$
W\left(m_{t} \mid\{o\}\right) \propto W\left(\{o\} \mid m_{t}\right)
$$

where $W\left(m_{t} \mid\{o\}\right)$ is the weight for the $t \bar{t}$ event with observed final state $\{o\}$ to have the top quark mass, $m_{t}$.

The relationship between weights given in Equation (7.9) is the key element of the $\nu \mathrm{WT}$ method. It allows us to produce $W\left(\{o\} \mid m_{t}\right)$ templates from Monte Carlo simulation with different mass hypotheses, and use those templates to extract the mass value and uncertainty from $W\left(m_{t} \mid\{o\}\right)$ when analyzing the data. The templates are made from many MC-generated events for both the signal, $t \bar{t}$, and the background processes. Although the number of background events is significantly reduced by applying the preselection cuts, it is still not negligible and thus needed to be accounted for. The mass extraction procedure is done with the help of a maximum likelihood technique taking account of these background events.

\subsubsection{Weight Calculation}

If the value of the top quark mass is given, the probability for a $t \bar{t}$ pair to decay to the observed final state, in principle, can be calculated analytically. Such probability is proportional to the integral of matrix element over final state parameters phase space

$$
P\left(\{o\} \mid m_{t}\right) \propto \frac{1}{\sigma_{o b s}^{t \bar{t}}\left(m_{t}\right)} \int f\left(q_{1}\right) f\left(q_{2}\right)|M|^{2} p(\{o\} \mid\{v\}) \delta^{4} \mathrm{~d}^{18}\{v\} \mathrm{d} q_{1} \mathrm{~d} q_{2}
$$


where $\{o\}$ is the set of 14 measured kinematic parameters and $\{v\}$ is the set of 18 momentum components of the six particles in the final state, $M$ is the matrix element for the $q \bar{q}$ or $g g$ interactions that result in the observed final state, $q_{i}$ denote the momentum fractions of the incident quarks in the proton and antiproton, and $f\left(q_{i}\right)$ are the parton distribution functions (PDFs) for finding a parton of a given flavor and longitudinal momentum fraction in the proton or antiproton. $p(\{o\} \mid\{v\})$ is the probability density to observe the values $\{o\}$ given the final state parameters $\{v\} . \sigma_{o b s}^{t \bar{t}}\left(m_{t}\right)$ is the normalization factor that represents the total observed $t \bar{t}$ cross-section for a given top quark mass. The mass constraints are imposed through the four-dimensional $\delta$-function

$$
\delta^{4}=\delta\left(m_{\ell_{1} \nu_{1}}-M_{W_{1}}\right) \times \delta\left(m_{\ell_{2} \nu_{2}}-M_{W_{2}}\right) \times \delta\left(m_{\ell_{1} \nu_{1} b_{1}}-m_{t}\right) \times \delta\left(m_{\ell_{2} \nu_{2} b_{2}}-m_{t}\right)
$$

where $m_{\ell_{i} \nu_{i}}$ is the invariant mass of the $i$-th lepton-neutrino pair, and $m_{\ell_{i} \nu_{i} b_{i}}$ invariant mass of the $i$-th lepton-neutrino pair and corresponding to it $i$-th $b$-quark.

The evaluation of the multidimensional integral in Equation (7.10) is very CPUintensive. Additionally to that, the above calculation deals in partons and the jets are far removed from this. Higher order effects, such as initial and final state gluon radiation, are also not taken into consideration by Equation (7.10). To obtain accurate top quark mass reconstruction, the higher order effects cannot be neglected and must be properly accounted for. Instead of calculating the probability using Equation (7.10), the $\nu \mathrm{WT}$ method seeks for simple weights that do not require significant computing time while retaining sensitivity to the value of the top quark mass.

The $\nu \mathrm{WT}$ method ignores measured $E_{T}^{x}$ and $E_{T}^{y}$ from the set of constraints. Instead, it assumes the rapidities of the two neutrinos $\eta_{\nu_{1}}, \eta_{\nu_{2}}$ from their predicted distributions. Taking into account $\eta_{\nu}=-\ln \left(\tan \frac{\theta_{\nu}}{2}\right)$ and therefore $\cos \left(\theta_{\nu}\right)=\frac{1-e^{-2 \eta}}{1+e^{-2 \eta}}$, the modified system of equations can be written as follows 


$$
\begin{gathered}
E_{\nu_{1}}=\left|\vec{p}_{\nu_{1}}\right|, \quad E_{\nu_{2}}=\left|\vec{p}_{\nu_{2}}\right| \\
p_{\nu_{1}}^{z}=\left|\vec{p}_{\nu_{1}}\right| \frac{1-e^{-2 \eta_{\nu_{1}}}}{1+e^{-2 \eta_{\nu_{1}}}}, \quad p_{\nu_{2}}^{z}=\left|\vec{p}_{\nu_{2}}\right| \frac{1-e^{-2 \eta_{\nu_{2}}}}{1+e^{-2 \eta_{\nu_{2}}}} \\
M_{W_{1}}^{2}=\left(E_{\ell_{1}}+E_{\nu_{1}}\right)^{2}-\left(\vec{p}_{\ell_{1}}+\vec{p}_{\nu_{1}}\right)^{2} \\
M_{W_{2}}^{2}=\left(E_{\ell_{2}}+E_{\nu_{2}}\right)^{2}-\left(\vec{p}_{\ell_{2}}+\vec{p}_{\nu_{2}}\right)^{2} \\
m_{t}=\left(E_{\ell_{1}}+E_{\nu_{1}}+E_{b_{1}}\right)^{2}-\left(\vec{p}_{\ell_{1}}+\vec{p}_{\nu_{1}}+\vec{p}_{b_{1}}\right)^{2} \\
m_{t}=\left(E_{\ell_{2}}+E_{\nu_{2}}+E_{b_{2}}\right)^{2}-\left(\vec{p}_{\ell_{2}}+\vec{p}_{\nu_{2}}+\vec{p}_{b_{2}}\right)^{2}
\end{gathered}
$$

where the value of the top quark mass $m_{t}$ is used as an additional constraint to permit a solution.

The system of equations (7.12) - (7.17) can be solved for the transverse momentum components of the two neutrinos $\left(p_{\nu_{1}}^{x}, p_{\nu_{1}}^{y}\right)$ and $\left(p_{\nu_{2}}^{x}, p_{\nu_{2}}^{y}\right)$. A two-fold ambiguity in the solution for each neutrino arises from the quadratic equations being solved and leads to the result for the two neutrino event having up to four solutions. Furthermore, every neutrino solution has an additional two-fold ambiguity due to pairing the charged leptons and $b$ jets that originate from the same top quark. Thus the number of possible solutions for the entire $t \bar{t}$ event, taking four-fold ambiguities for the two neutrinos and two possible jet assignments, can be up to eight.

For each solution, the $\nu \mathrm{WT}$ method assigns the weight $w_{i}$ that characterizes the consistency of the resulting solutions with the top quark mass assumption $m_{t}$ and the choice of neutrino rapidities $\eta_{\nu_{1}}$ and $\eta_{\nu_{2}}$. It does this by comparing the solved neutrino momenta to the observed $E_{T}^{x}$ and $E_{T}^{y}$,

$$
w_{i}=\exp \left(\frac{-\left(E_{T}^{x}-p_{\nu_{1}, i}^{x}-p_{\nu_{2}, i}^{x}\right)^{2}}{2 \sigma_{x}^{2}}\right) \times \exp \left(\frac{-\left(E_{T}^{y}-p_{\nu_{1}, i}^{y}-p_{\nu_{2}, i}^{y}\right)^{2}}{2 \sigma_{y}^{2}}\right)
$$


where $i$ denotes the corresponding solution, $\sigma_{x}$ and $\sigma_{y}$ are the $\mathbb{E}_{T}$ detector resolution in $x$ and $y$ directions. To calculate an event weight for a given top quark mass and particular choice of neutrino rapidities, the sum is taken over all possible neutrino momentum solutions with different jet assignments

$$
w\left(\eta_{\nu_{1}}, \eta_{\nu_{2}} \mid m_{t}\right)=\sum_{i}^{N_{s o l n}} w_{i}
$$

The neutrino rapidity choices are not equally likely. An example of the neutrino rapidity distribution modeled by ALPGEN is shown in Figure 7.2. Neutrinos with rapidities in the central region are more likely to be produced in the event than those in the forward region. When normalized to one, the neutrino rapidity distribution becomes the probability density distribution of neutrino rapidities, $\rho\left(\eta_{\nu}\right)$. The probability density distribution can be approximated by a single Gaussian function. A Gaussian function with a width dependent on the top quark mass is a very good approximation.

The total weight $W\left(\{o\} \mid m_{t}\right)$ for an event with assumed top quark mass, $m_{t}$, and observed final state $\{o\}$ is obtained by integrating over neutrino rapidities with corresponding probability distribution function $\rho\left(\eta_{\nu_{1}}\right)$ and $\rho\left(\eta_{\nu_{2}}\right)$

$$
W\left(\{o\} \mid m_{t}\right)=\iint w\left(\eta_{\nu_{1}}, \eta_{\nu_{2}} \mid m_{t}\right) \rho\left(\eta_{\nu_{1}}\right) \rho\left(\eta_{\nu_{2}}\right) \mathrm{d} \eta_{\nu_{1}} \mathrm{~d} \eta_{\nu_{2}}
$$

Scanning over the range of different top quark masses from $80 \mathrm{GeV}$ to $330 \mathrm{GeV}$ in 1 $\mathrm{GeV}$ increments, the $\nu \mathrm{WT}$ produces the weight distribution for every individual event. An example of the weight distribution for an event from the $t \bar{t}$ MC sample with $m_{t}=172$ $\mathrm{GeV}$ is shown in Figure 7.3. Figure 7.4 shows the weight distribution averaged over all events for samples with input top quark masses of $160 \mathrm{GeV}, 170 \mathrm{GeV}$ and $180 \mathrm{GeV}$. Due to resolution effects and jet permutation assignments, the average distribution has a broader peak. Initial and final state radiation add a high-mass tail and shift the peak 


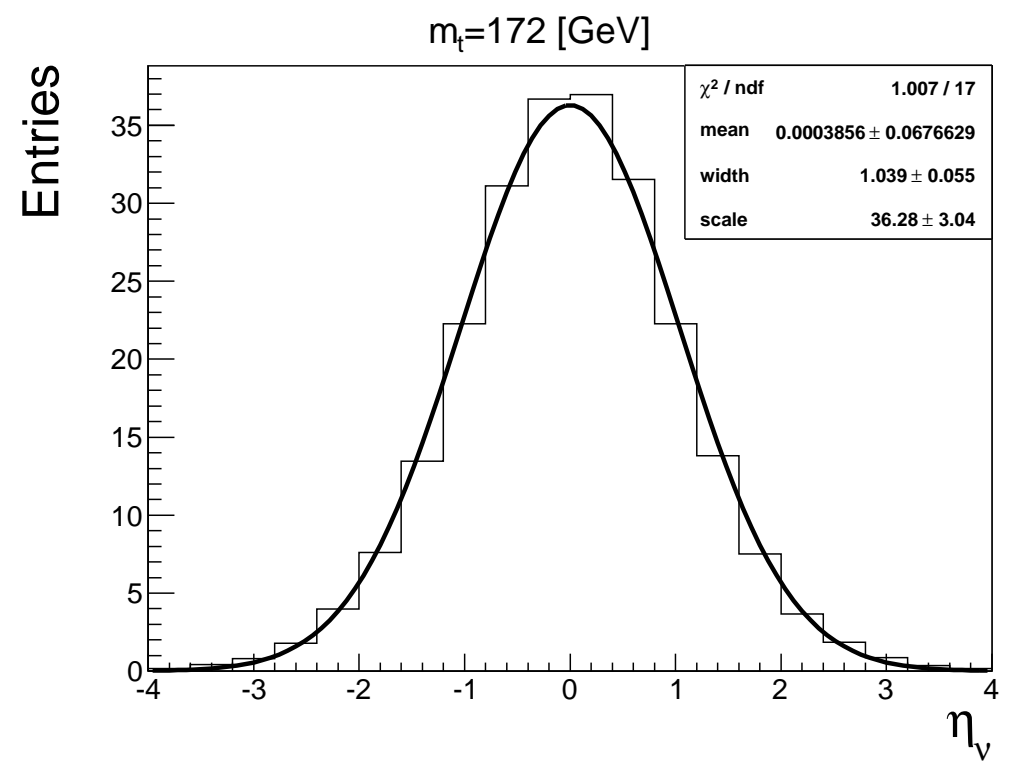

Figure 7.2. Example of neutrino rapidity distribution with the top quark mass $m_{t}=172$ $\mathrm{GeV}$ as simulated by ALPGEN. The smooth black curve is a Gaussian fit.

of the distribution from the input mass.

\subsubsection{Neutrino Pseudorapidity Choices}

To compute the integral in Equation (7.20) numerically, I applied the midpoint integration approach. For each integral, the space of integration is divided in number of bins and the sum over all bins of integrand values at the center of a bin times bin size is taken

$$
W\left(\{o\} \mid m_{t}\right)=\sum_{i=1}^{N} \sum_{j=1}^{N} w\left(\eta_{\nu_{1}}^{i}, \eta_{\nu_{2}}^{j} \mid m_{t}\right) \rho\left(\eta_{\nu_{1}}^{i}\right) \rho\left(\eta_{\nu_{2}}^{j}\right) \Delta \eta_{\nu_{1}}^{i} \Delta \eta_{\nu_{2}}^{j}
$$

where $N$ is the number of bins into which the space is split. 


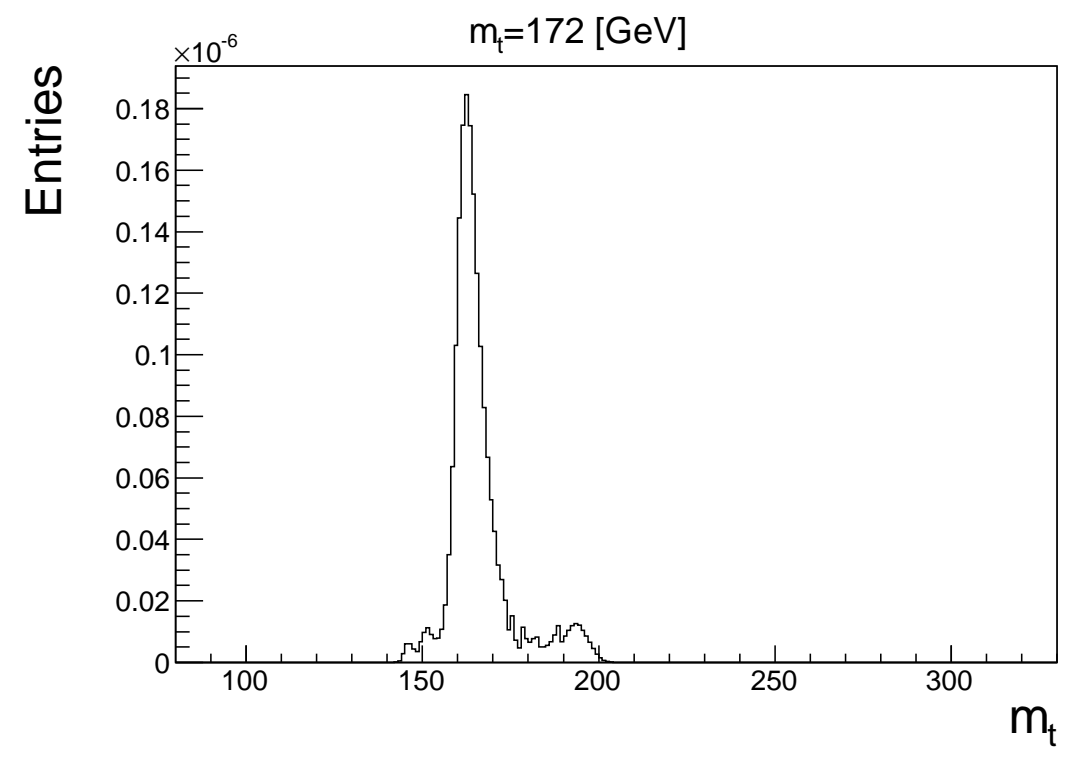

Figure 7.3. Example of the weight distribution for simulated $t \bar{t}$ event with $m_{t}=172$ $\mathrm{GeV}$.

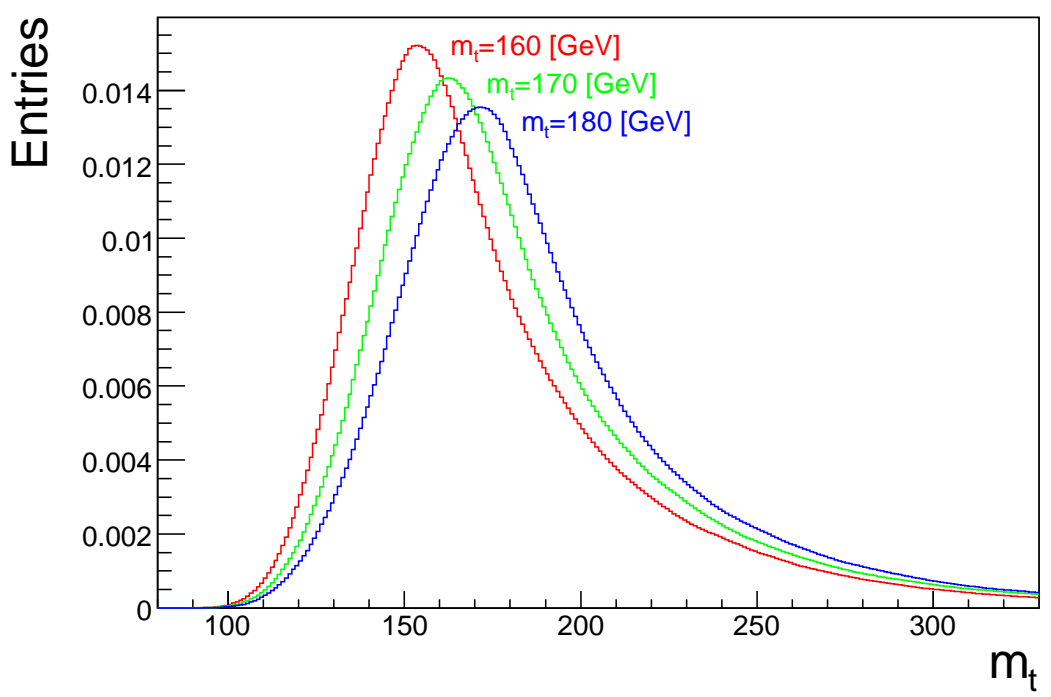

Figure 7.4. The weight distribution averaged over all events in MC $t \bar{t}$ sample of masses $160 \mathrm{GeV}$ (red), $170 \mathrm{GeV}$ (green) and $180 \mathrm{GeV}$ (blue). 
Shapes of the weight distribution obtained with a different binning of the integration space differ significantly. Figure 7.5 shows a single Monte Carlo event weight distribution with different numbers of rapidity choices. The 10 bin choice has been used in the previous measurement of the top quark with the $\nu \mathrm{WT}$ method [109]. The granularity of the neutrino rapidity distribution affects CPU load directly. The time needed to compute the weight distribution grows as $n^{2}$, where $n$ is the bin granularity factor. For example, increasing the number of bins in the neutrino rapidity distribution by factor of 3 would require 9 times more time. Changing the binning from 10 to 70 bins, the shapes of the weight distribution change dramatically. However, a further increase in the binning from 70 to 200 does not result in a significant change of the distribution shapes. The analysis presented in this thesis was performed with 30 rapidity choices for each neutrino. It provides the benefit of the 70 choice scenario and allows to perform the required calculations in a reasonable time. The processing time for all $\mathrm{MC}$ signal samples is about 3 days on 600 nodes (each node is equipped with $2.2 \mathrm{GHz}$ processor and 2GB memory). To improve the accuracy when integrating over neutrino rapidities, the bins of rapidities were chosen such that all bins contain equal statistics. Varying bin size and equal values in the bins ensure better approximation of the integral in Equation (7.20) by the double-sum from Equation (7.21).

Finely integrating over neutrino rapidities removes the necessity to smear measured kinematics within their experimental resolutions. In the previous measurement [109], smearing the momenta was inevitable to attain the stability of the main momenta of the weight distribution - mean and root-mean-square (or width). By fluctuating jets and leptons transverse momenta according to their known resolutions, the resulting weight distribution becomes smoother and the range of assumed top quark masses with a nonzero solution increases. With the finer binning in the neutrino rapidity, the integral calculation achieves similar results in stability of distribution momenta as the smearing 
approach used in the previous measurement.

\subsubsection{Neutrino Pseudorapidity Modeling}

The Gaussian function used to describe the MC-simulated distribution of neutrino rapidity slightly varies with the top quark mass. To analyze the dependence of the moments of the Gaussian on the mass, the neutrino rapidity distribution was simulated for the different top quark masses. I fit each simulated distribution with the Gaussian function and extracted its width and mean values. The mean value of the Gaussian is mass-independent and equals zero due to $p \bar{p}$ collider symmetry. The width of the Gaussian shows a weak dependence on the mass. Figure 7.6 shows the width versus input top quark mass with the linear fit applied. Good $\chi^{2}$ per degree of freedom for the fit suggests that the width dependence on the top quark mass can be parameterized with the linear function,

$$
\sigma_{\eta}=-0.001 m_{t}+1.17
$$

Equation (7.22) is employed in the analysis of this thesis for sampling neutrino rapidity.

\subsection{Maximum Likelihood Method}

The top quark mass is determined by comparing the weight distributions for the detector data with the weight distributions from Monte Carlo samples simulated at

different values of the top quark mass. To find the top quark mass at which the data shows the best agreement with the Monte Carlo simulation, the maximum likelihood technique is employed.

The likelihood function is given as

$$
L\left(m_{t}\right)=\prod_{i=1}^{N_{\text {data }}} \frac{\bar{n}_{s} h_{s}\left(\vec{x}_{i} \mid m_{t}\right)+\bar{n}_{b} h_{b}\left(\vec{x}_{i}\right)}{\bar{n}_{s}+\bar{n}_{b}}
$$


where $i$ runs over all events in the data sample, $N_{\text {data }}$ is the total number of events, $\bar{n}_{s}, \bar{n}_{b}$ are the expected number of signal and background events. The $\bar{n}_{b}$ is estimated from Monte Carlo simulation and $\bar{n}_{s}=N_{\text {data }}-\bar{n}_{b}$. The $\vec{x}$ is a set of $N$ input variables $\left(x_{1}, x_{2}, \ldots, x_{N}\right)$ characterizing the weight distribution of an event. The $h_{s}\left(\vec{x}_{i} \mid m_{t}\right)$ is the $N$-dimensional probability density histogram for signal and $h_{b}\left(\vec{x}_{i}\right)$ for background events. In the context of the method, the $h_{s}\left(\vec{x}_{i} \mid m_{t}\right)$ and $h_{b}\left(\vec{x}_{i}\right)$ are also referred as the templates. Both templates are constructed from the input variables calculated with Monte Carlo simulated events. In addition to the input variables, the signal template has the extra dimension $m_{t}$ since the $N$-dimensional distribution is mass dependent.

The set of variables $\vec{x}$ can be picked in more than one way. For example, it can be as simple as one parameter - the peak of the weight distribution, or more complicated as the integrated weight in bins of a coarsely binned weight distribution. Some choices of variables provide a better discriminating power than others. It is most effective when the variables are the most dependent on the top quark mass. In addition, the more the variables are uncorrelated with each other, the better discrimination can be achieved.

Without losing too much information, and following the choice of the variables from [109], the mean $\left(\mu_{w}\right)$ and the width $\left(\sigma_{w}\right)$ of a weight distribution are chosen as input variables $\vec{x}$ to the likelihood function in the analysis of this thesis. $\mu_{w}$ is highly correlated with the value of the top quark mass. $\sigma_{w}$ has much smaller correlation but provides additional information and increases the overall sensitivity to the top quark mass.

For computational convenience, we consider the logarithm of the likelihood function. The logarithm of the likelihood function is also called the log-likelihood. In many analyses at DØ, the log-likelihood is taken with minus sign in front. Thus the task of maximization of the likelihood is equivalent to the minimization of the negative loglikelihood. The negative log-likelihood is plotted versus top quark mass hypotheses and fitted to a parabola. The value of $m_{t}$ at the minimum of the parabola minimizes the 
negative log-likelihood and therefore gives an estimate of most probable top quark mass $m_{t}^{\text {meas }}$. The estimate of the top quark mass with Maximum Likelihood Method is aslo called the top quark mass estimator.

The top quark mass estimator and the statistical uncertainty of the measurement is based on the assumption that the likelihood function has a Gaussian shape around its maximum. The Gaussian shape of the likelihood indicates that the values of the measured top quark mass are spread around its true value according to the normal distribution [110]. The Gaussian likelihood assumed can be written in the following form

$$
L\left(m_{t}\right)=A \exp \left(-\frac{\left(m_{t}-\mu\right)^{2}}{2 \sigma^{2}}\right)
$$

where $A$ is normalization coefficient. The negative log-likelihood then is given as

$$
-\ln \left(L\left(m_{t}\right)\right)=\frac{\left(m_{t}-\mu\right)^{2}}{2 \sigma^{2}}+C
$$

where $C \equiv-\ln (A)$.

The right-hand side of Equation (7.25) is a parabola and reaches its minimum value $C$ at the point $m_{t}=\mu$. Thus the most probable value of the top quark mass is $\mu$, and according to the normal distribution error definition, $\sigma$ is the statistical uncertainty of the measurement, i.e. the value of the top quark mass is known to be $m_{t}=\mu \pm \sigma$ at $68.2 \%$ confidence. At the points $m_{t}=\mu \pm \sigma$ the negative log-likelihood rises

$$
-\ln (L(\mu \pm \sigma))=\frac{(\mu \pm \sigma-\mu)^{2}}{2 \sigma^{2}}+C=\frac{( \pm \sigma)^{2}}{2 \sigma^{2}}+C=0.5+C
$$

to 0.5 units more than its minimum value $C$. For a parameter with a gaussian variation, half the width of the parabola where the negative log-likelihood rises to 0.5 units more than its minimum value provides the statistical uncertainty $\sigma^{\text {meas }}$. Thus the negative 
log-likelihood allows to obtain complete measurement of the top quark mass, i.e. $m_{t}^{\text {meas }} \pm$ $\sigma^{\text {meas }}$. In practice, however, the value of $\sigma^{\text {meas }}$ is determined from the fit of the likelihood distribution to a parabola. If the general form of a parabola is given as $f(x)=a x^{2}+$ $b x+c$ then comparing it to the right-hand side of Equation (7.25)

$$
a m_{t}^{2}+b m_{t}+c=\frac{\left(m_{t}-\mu\right)^{2}}{2 \sigma^{2}}+C
$$

it is easy to find that

$$
\sigma=\frac{1}{\sqrt{2 a}}
$$

If the parameters $a, b$ and $c$ are obtained from the fit, then the statistical uncertainty calculated with Equation(7.28) gives a value of $\sigma^{\text {meas }}$.

Since a Gaussian function does not approximate the tails of the likelihood distribution well, it is necessary to restrict the fit range. The fit is applied to points near the observed minimum of the likelihood $-\ln (L(\mu))=C$. To obtain the best possible agreement between measured top quark mass and a corresponding top quark mass hypothesis, different ranges of mass points were tested and an optimal value was chosen for the measurement on data.

\subsubsection{Probability Density Templates}

To build the signal and background templates needed for the likelihood function calculation, Monte Carlo simulated samples are used. The signal samples are generated for the $t \bar{t}$ dilepton decay mode $t \bar{t} \rightarrow \ell \ell^{\prime}+\bar{\nu} \nu^{\prime}+b \bar{b}$ with different top quark mass hypotheses. The mass hypothesis in the context of the templates is also known as input or test top quark mass. The range of input top quark masses from $130 \mathrm{GeV}$ to $210 \mathrm{GeV}$ with increment of $5 \mathrm{GeV}$ is used. The physics background samples include $Z / \gamma^{*} \rightarrow \tau \tau$ with $\tau \rightarrow e, \mu$ and diboson $(W W, W Z, Z Z)$ production. The instrumental background 
cannot be easily simulated and rather is obtained from the detector data.

Two parameters are used to characterize the event weight distribution - the mean $\mu_{w}$ and the width $\sigma_{w}$. The template for simulated signal events $h_{s}\left(\mu_{w}, \sigma_{w} \mid m_{t}\right)$ is defined as a 3-dimensional histogram of input top quark mass, $m_{t}$, vs. $\mu_{w}$ and $\sigma_{w}$. The back-

ground template $h_{b}\left(\mu_{w}, \sigma_{w}\right)$ is one dimension less than a signal template since there is no associated top quark mass hypothesis. Thus $h_{b}\left(\mu_{w}, \sigma_{w}\right)$ is just defined as 2-dimensional histogram of $\mu_{w}$ and $\sigma_{w}$ and contains the sums of all backgrounds according to their expected yields. Both $h_{s}\left(\mu_{w}, \sigma_{w} \mid m_{t}\right)$ and $h_{b}\left(\mu_{w}, \sigma_{w}\right)$ are normalized as follows

$$
\begin{gathered}
\int h_{s}\left(\mu_{w}, \sigma_{w} \mid m_{t}\right) \mathrm{d} \mu_{w} \mathrm{~d} \sigma_{w}=1 \\
\int h_{b}\left(\mu_{w}, \sigma_{w}\right) \mathrm{d} \mu_{w} \mathrm{~d} \sigma_{w}=1
\end{gathered}
$$

where the integral is taken over the whole physically allowed region of $\mu_{w}$ vs $\sigma_{w}$. The two-dimensional slice of the three-dimensional signal template for input top quark mass $m_{t}=170 \mathrm{GeV}$ is shown in Figure 7.7. Figure 7.8 shows the combined background probability density template.

\subsection{Testing and Calibration using Pseudoexperiments}

The presence of the background shifts the measured value of the top quark mass from its true value. In addition to that, the $\nu \mathrm{WT}$ method relies on a number of assumptions. Thus for instance, the likelihood may not be well described by a Gaussian, or the neutrino rapidities are not distributed according to the Standard Model expectations. To correct for such effects, the final result needs to be calibrated.

To verify the precision and performance of the method as well as to derive a calibration factor as a function of input mass, the pseudoexperiment testing technique is used. 
Similarly to data, the value of the top quark mass is extracted in pseudoexperiments (PEs) using Maximum Likelihood Method. A pseudoexperiment is formed from MC events and has the same size as the analyzed dataset. An array of pseudoexperiments is called an ensemble. Since to test the method, different ensembles of PEs might be used, an ensemble testing is often a synonym to the pseudoexperiment testing. Figure 7.9 shows $-\ln (L)$ for a randomly selected from an ensemble pseudoexperiment for the $e \mu$ channel. The MC events for a PE are drawn randomly from signal and background samples, and the composition of the pseudoexperiments on average corresponds to that expected in data. The number of signal and background events in a PE is obtained according to a Poisson distribution. The mean for the Poisson distribution is generated randomly from a Gaussian distribution. For a given process, the Gaussian has its mean equal to the expected yield and the standard deviation equals the statistical uncertainty on the expected yield. The $t \bar{t}$ signal and background processes are fluctuated independently and only those scenarios yielding the number of events in the dataset are selected for the testing.

\subsubsection{Mass Measurement Calibration}

To validate the method with ensemble testing approach, the number of pseudoexperiments has to be large. A thousand pseudoexperiments are performed for each top quark mass hypothesis. The background yield and composition are independent of the mass hypothesis. The distribution of the top quark mass estimators for a given top quark mass hypothesis is obtained by performing the negative log-likelihood minimization for each pseudoexperiment. The mean of the distribution is plotted against the input top quark mass value and a linear fit is performed. The fitted line provides a calibration curve. The calibration is parameterized as follows 


$$
m_{t}^{\text {fit }}\left(m_{t}\right)=\alpha\left(m_{t}-170\right)+\beta+170
$$

where $m_{t}^{f i t}$ is the fitted value and $m_{t}$ is the input top quark mass. The values of parameters slope, $\alpha$, and offset, $\beta$, in ideal case are equal one and zero respectively.

The statistical uncertainty on the calibration points is the root-mean-square of the corresponding mass estimator distribution taken from 1000 PEs. Due to the limited statistics of Monte Carlo samples, most events can appear more than once in different PEs in the ensemble. This results in the correlation between the PEs. Thus to obtain an improved precision of the statistical uncertainty, the resampling correction [111], [112] was applied. An example of calibration curve for ee channel is shown in Figure 7.10. The effects causing a high $\chi^{2}$ in the calibration fit are described in Section 7.4.1.1.

To calibrate the mass measurement on real data, $m_{t}^{\text {meas }}$, the inverse of Equation (7.31) is applied

$$
m_{t}^{\text {meas }, \text { calib }}=\frac{m_{t}^{\text {meas }}-\beta-170}{\alpha}
$$

where $m_{t}^{\text {meas, calib }}$ is the measurement of the top quark mass in the data corrected for the possible biases of the method. The value of $m_{t}^{\text {meas, calib }}$ is the best top quark mass estimate of the true value.

\subsubsection{High $\chi^{2}$ of Calibration Curve}

The linear fit to calibration points from the pseudoexperiment studies show a high $\chi^{2}$. This could be a result of several effects such as large oversampling of the background events due to lower backgrounds statistics as compared to the signal events, wide variation of ALPGEN weights within MC samples, and point-to-point fluctuations in the calibration plots due to the finite template statistics. The detailed evaluation of the impact on calibration curve $\chi^{2}$ from each of these effects is provided in Ref. [100]. 
To evaluate the effect from large oversampling of the background events, the calibrations for all three channels and the combination were determined in signal-only configuration of pseudoexperiments. The values of the slope and offset are found to be stable while $\chi^{2}$ still remains high.

The effect of the finite template statistics is examined by setting all ALPGEN weights to a constant value. This would allow us to sample events in the pseudoexperiments for signal-only configuration roughly equally. After running the pseudoexperiment testing, the $\chi^{2}$ from the calibration plots are still found to be high. To establish that this is a result of the the finite template statistics, the calibrated and input top quark masses are calculated. The low-statistics signal samples show high deviations while for high statistics samples it is low. To exclude impact from faulty MC simulation or some other biases, $\nu \mathrm{WT}$ moments are considered. Thus, for example, the mean value of weight distribution $\mu_{w}$ for every event is not affected by template statistics. The average $\mu_{w}$ vs input top quark mass is fitted with a linear function. The deviation of $\left\langle\mu_{w}\right\rangle$ from this function for each mass point is found to be very small. This indicates that the large fluctuations in the calibrated top quark masses are due to lower template statistics.

The effect from variation of ALPGEN weights is evaluated by setting all weights to default values. The pseudoexperiment testing showed that contribution to the $\chi^{2}$ from the variation of ALPGEN weights is at a lesser level than from the template statistics. Thus, the largest contribution to the $\chi^{2}$ comes from the effect of finite template statistics, with smaller contributions from varying event weights and background oversampling.

To estimate the effect of finite template statistics, the pseudoexperiment testing is performed by taking signal-only pseudoexperiments with ALPGEN weights using the $m_{t}=172.5 \mathrm{GeV}$ sample. For each pseudoexperiment, signal template is sampled within its uncertainties. The root-mean-square (RMS) of the calibrated mass measurements is calculated. The average RMS for 100 pseudoexperiments yields $0.3 \mathrm{GeV}$. This variation 
is taken to be an additional uncertainty and added in quadrature with the statistical uncertainties at each mass point in the calibration plots. The calibration points with the template statistics accounted for and linear fit are shown separately for each channel in Figure 7.11.

\subsubsection{Statistical Uncertainty Calibration}

The statistical uncertainty extracted with the negative log-likelihood approach is also biased due to the same residual effects and assumptions described above. Therefore, the statistical uncertainty needs to be calibrated. This section describes the method I used to calibrate the statistical uncertainty.

Subsection 7.4.2.1 describes the correction due to the top quark mass calibration. Consequently, the tests of the calibrated statistical uncertainty are described in the subsection 7.4.2.2 and the final correction is given.

\subsubsection{Mass Calibrated Correction}

According to the likelihood approach, the statistical uncertainty measured in a pseudoexperiment is defined as follows

$$
\sigma^{\text {meas }}=\left|m_{t}^{\text {meas }}-m_{t, 1 / 2}^{\text {meas }}\right|
$$

where $m_{t}^{\text {meas }}$ is the top quark mass estimate and $m_{t, 1 / 2}^{\text {meas }}$ is the value for which $-\ln L\left(m_{t}\right)$ rises to 0.5 units more than its minimum value. Then the calibrated measured uncertainty is given as

$$
\sigma^{\text {meas, }} \text { calib }=\left|m_{t}^{\text {meas, } \text { calib }}-m_{t, 1 / 2}^{\text {meas }, \text { calib }}\right|
$$

where $m_{t}^{\text {meas,calib }}$ and $m_{t, 1 / 2}^{\text {meas, calib }}$ are calibrated values of $m_{t}^{\text {meas }}$ and $m_{t, 1 / 2}^{\text {meas }}$ correspondingly. 
To derive a calibrating equation for statistical uncertainty, it is assumed that $m_{t, 1 / 2}^{\text {meas }}$ can be calibrated using the formula analogous to Equation (7.32). Thus the following calibrating formulas for $m_{t}^{\text {meas }}$ and $m_{t, 1 / 2}^{\text {meas }}$ can be written

$$
\begin{aligned}
m_{t}^{\text {meas }, \text { calib }} & =\frac{m_{t}^{\text {meas }}-\beta-170}{\alpha} \\
m_{t, 1 / 2}^{\text {meas, calib }} & =\frac{m_{t, 1 / 2}^{\text {meas }}-\beta-170}{\alpha}
\end{aligned}
$$

By plugging in Equation (7.35) and (7.36) to (7.34), and employing definition of $\sigma^{\text {meas }}$ as in Equation (7.33), the relationship between measured and calibrated statistical uncertainties becomes

$$
\sigma^{\text {meas }, \text { calib }}=\frac{\sigma^{\text {meas }}}{\alpha}
$$

The resulting slopes, offsets are given in Table 7.1.

Table 7.1. The slope $(\alpha)$ and offset $(\beta)$ calibration parameters with the statistical uncertainty for the dilepton channels and the combination.

\begin{tabular}{c|cc}
\hline \hline Channel & $\alpha$ & $\beta$ \\
\hline \hline$e e$ & $0.976 \pm 0.014$ & $0.030 \pm 0.158$ \\
\hline$e \mu$ & $0.973 \pm 0.012$ & $0.425 \pm 0.135$ \\
\hline$\mu \mu$ & $1.038 \pm 0.022$ & $0.489 \pm 0.234$ \\
\hline combined & $1.018 \pm 0.012$ & $0.416 \pm 0.133$ \\
\hline \hline
\end{tabular}




\subsubsection{Pull Width Calibration}

To test the calibrated statistical uncertainty, $\sigma^{\text {meas, calib }}$, estimated from the likelihood distribution, the ensemble testing technique is applied again. Thus the correctness of the uncertainty is evaluated with the pull variable

$$
p \equiv \frac{m_{t}^{\text {meas,calib }}-m_{t}}{\sigma^{\text {meas }, \text { calib }}}
$$

where $m_{t}$ is the input top quark mass. The pull variable is calculated for each individual pseudoexperiment and the ensemble is used to produce a pull distribution for a given top quark mass hypothesis. An example of a pull distribution for the input top quark mass $m_{t}=170 \mathrm{GeV}$ is given in Figure 7.12. Ideally, the pull distribution of any top quark mass hypothesis should be a Gaussian with the mean around zero and the width close to one. The derivation of the probability density function for an ideal pull distribution is provided in Appendix $\mathrm{C}$ and the non-ideal case is detailed in Appendix C.

The width of a pull distribution is plotted against the top quark mass hypotheses. Statistical uncertainty on the width value at every input top quark mass point is just the root-mean-square of corresponding pull distribution corrected using the resampling technique [112]. A calibration is obtained by fitting the pull vs. $m_{t}$ to a constant,

$$
p\left(m_{t}\right)=\lambda
$$

where $\lambda$ is the only fit parameter. An example of a pull width calibration and the fitted function are shown in Figure 7.13. $\lambda$ is the value of the pull distribution width averaged over many pseudoexperiments and mass hypotheses.

In the ideal case, the width of a pull distribution at every input mass point is equal one and therefore $\lambda=1$ as well. In practice however, the parameter $\lambda$ is slightly different from one. A non-unitary value of $\lambda$ indicates that the calibrated uncertainty, $\sigma^{\text {meas, calib }}$, 
estimated from the likelihood distribution is either over-or-under estimated and therefore requires additional correction.

The pull-corrected calibrated statistical uncertainty $\sigma^{\text {calib,corr }}$ is obtained from the $\sigma^{\text {meas, calib }}$ simply multiplying the latter by $\lambda$

$$
\sigma^{\text {calib,corr }}=\lambda \sigma^{\text {meas }, \text { calib }}
$$

The derivation of Equation (7.40) is provided in Appendix D.

Combining Equation (7.37) and (7.40), the pull-corrected calibrated uncertainty $\sigma^{\text {calib }, \text { corr }}$ is related to the measured statistical uncertainty $\sigma^{\text {meas }}$ by

$$
\sigma^{\text {calib, corr }}=\frac{\lambda}{\alpha} \sigma^{\text {meas }}
$$

Equation (7.41) is employed to correct the statistical uncertainty measured on the actual dataset with the likelihood technique of the $\nu \mathrm{WT}$ method. The resulting pull widths are given in Table 7.2 . 
Table 7.2. The pull-calibrating correction $(\lambda)$ with the statistical uncertainty for the dilepton channels and the combination.

\begin{tabular}{c|c}
\hline \hline Channel & $\lambda$ \\
\hline \hline$e e$ & $1.006 \pm 0.012$ \\
\hline$e \mu$ & $1.028 \pm 0.013$ \\
\hline$\mu \mu$ & $1.056 \pm 0.032$ \\
\hline combined & $1.052 \pm 0.013$ \\
\hline \hline
\end{tabular}




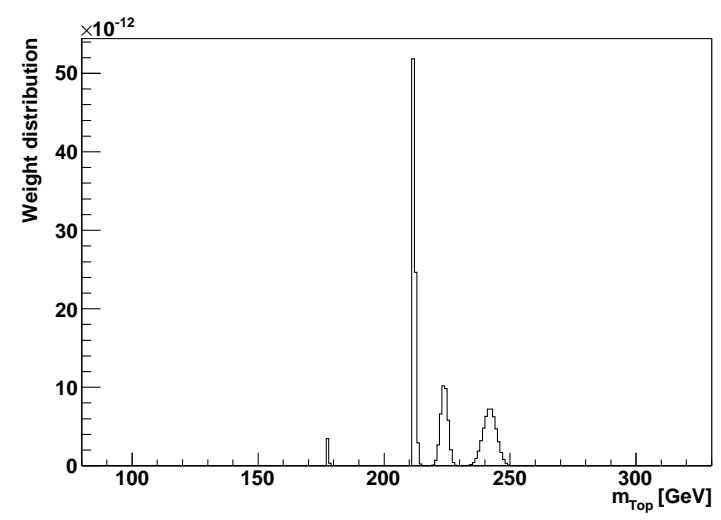

(a)

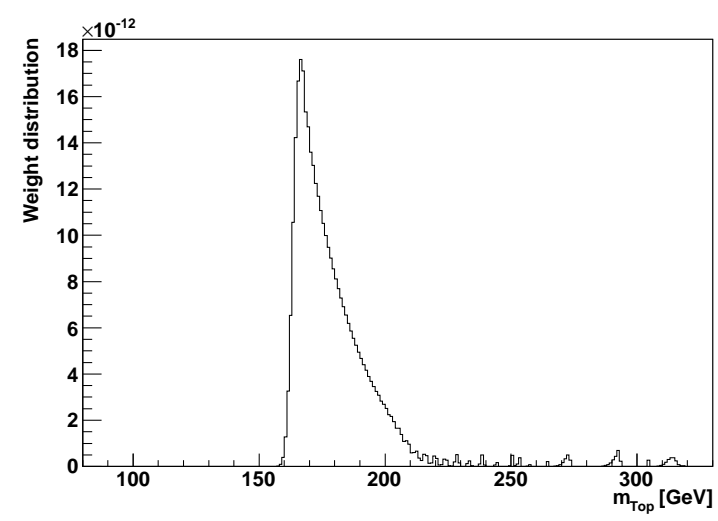

(c)

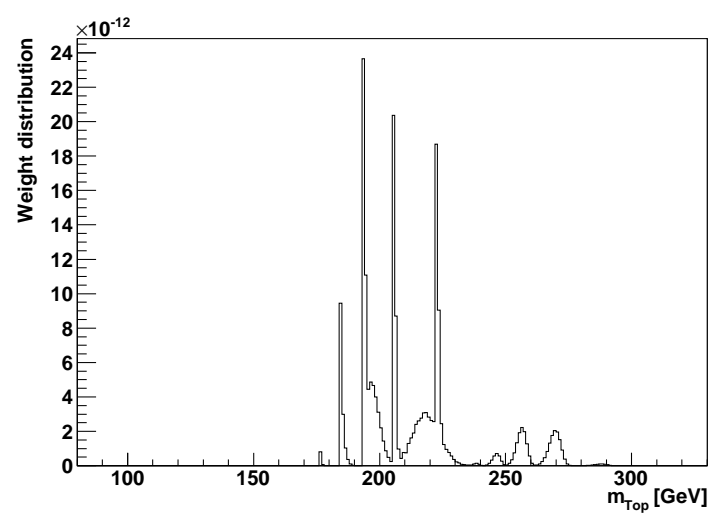

(b)

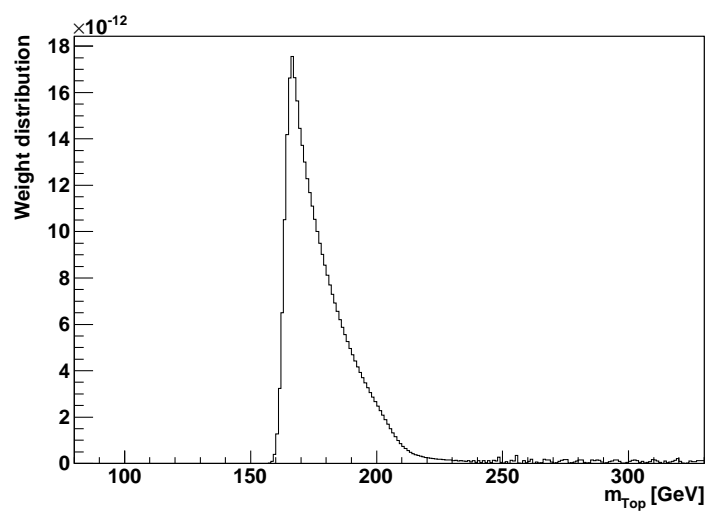

(d)

Figure 7.5. Example of the weight distributions obtained with different numbers of rapidity bins: (a) $N=10$, (b) $N=30$, (c) $N=70$, (d) $N=200$ 


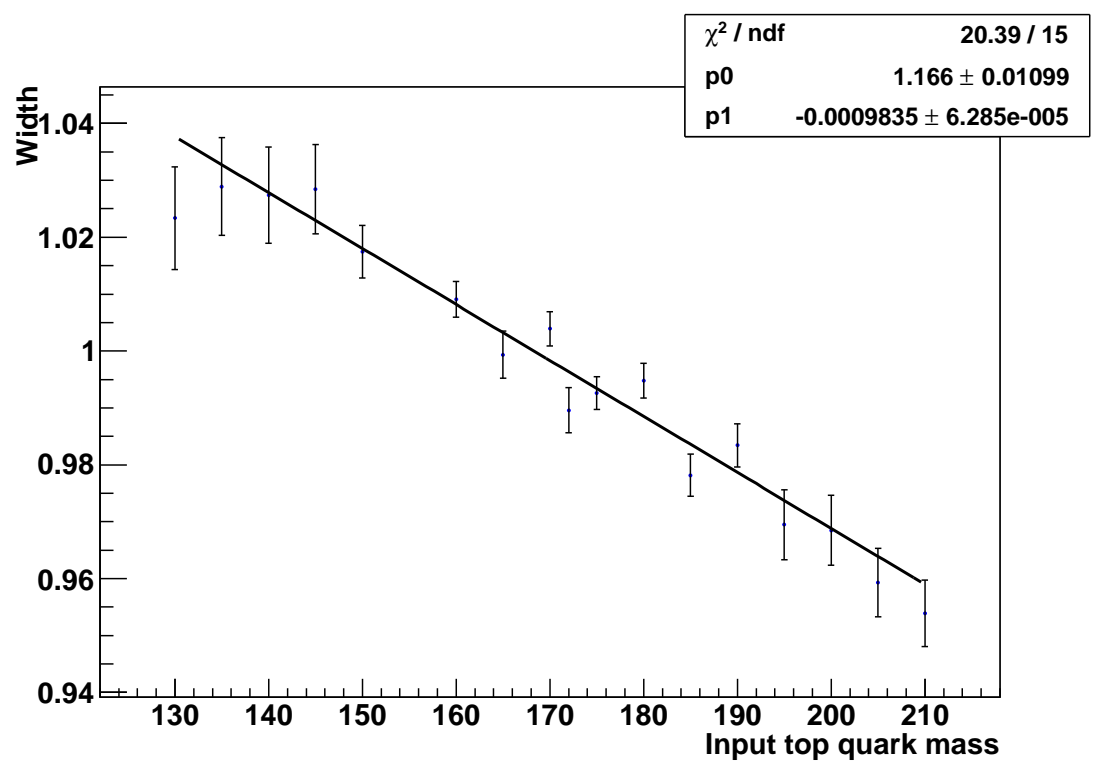

Figure 7.6. Width of the Gaussian fit to the neutrino rapidity distribution as a function of the top quark mass. The black line is a linear fit. 


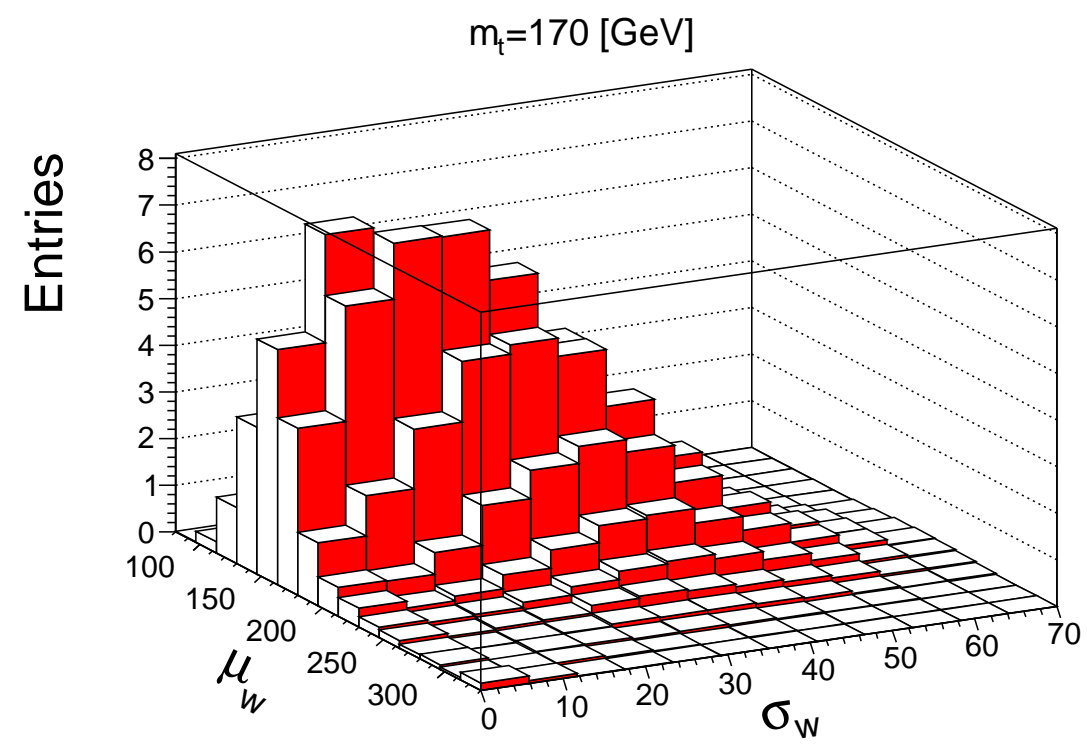

Figure 7.7. Slice of the probability density template $h_{s}\left(\mu_{w}, \sigma_{w} \mid m_{t}\right)$ for $m_{t}=170 \mathrm{GeV}$ for $e \mu$ channel.

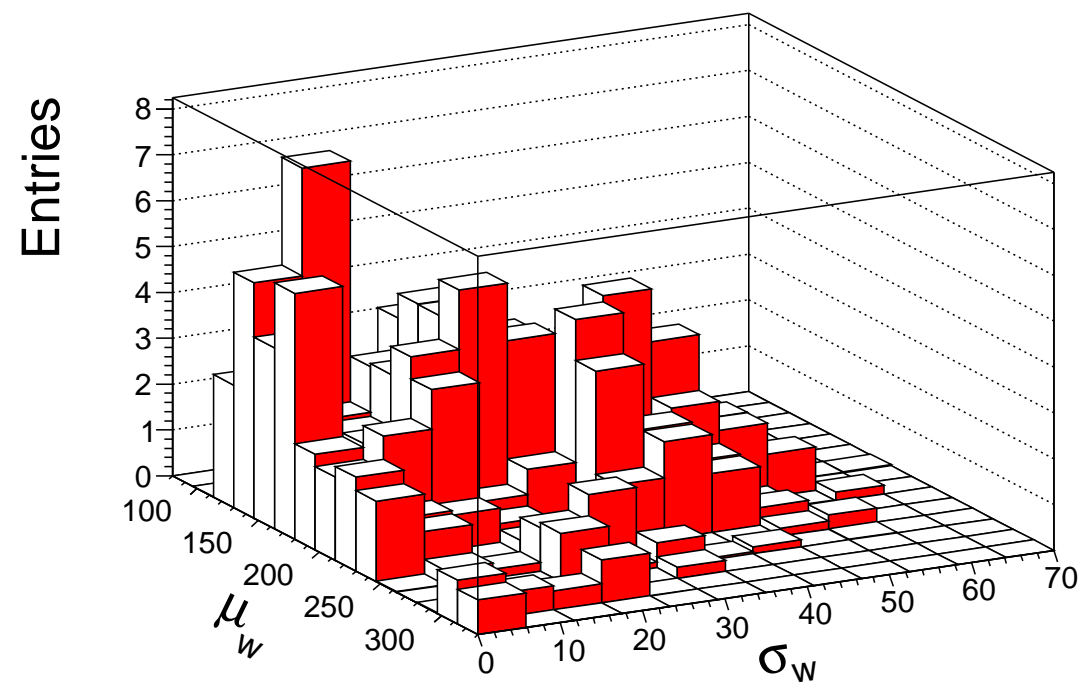

Figure 7.8. The combined background probability density template $h_{b}\left(\mu_{w}, \sigma_{w}\right)$ for $e \mu$ channel. 


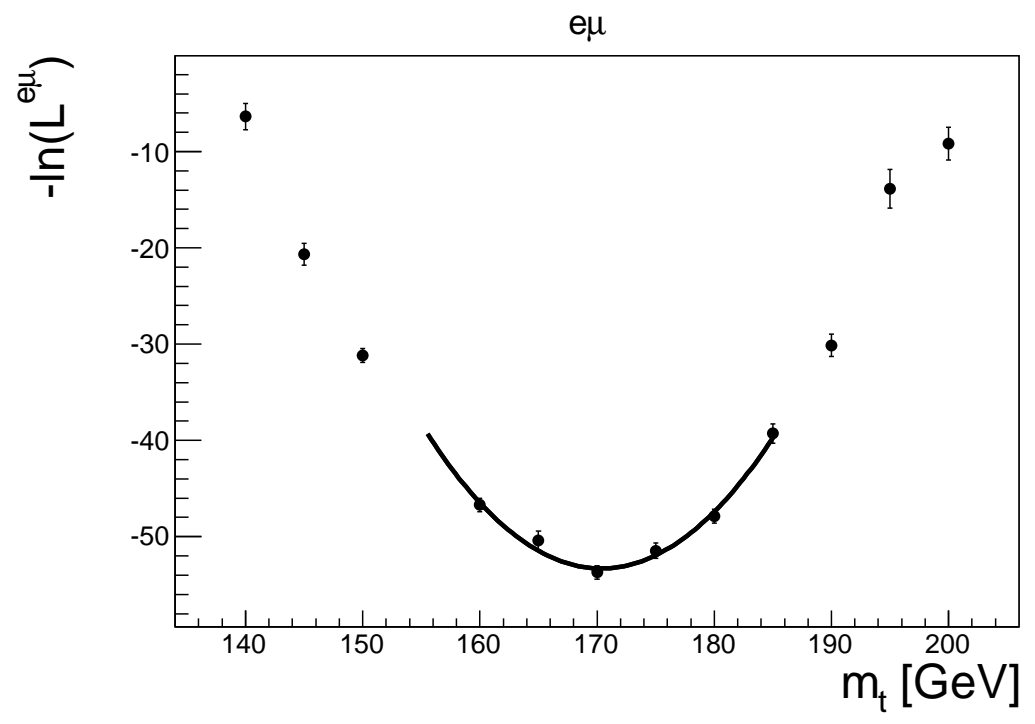

Figure 7.9. $\quad-\ln (L)$ for a $\mathrm{MC}$ simulated pseudoexperiment for $e \mu$ channel.

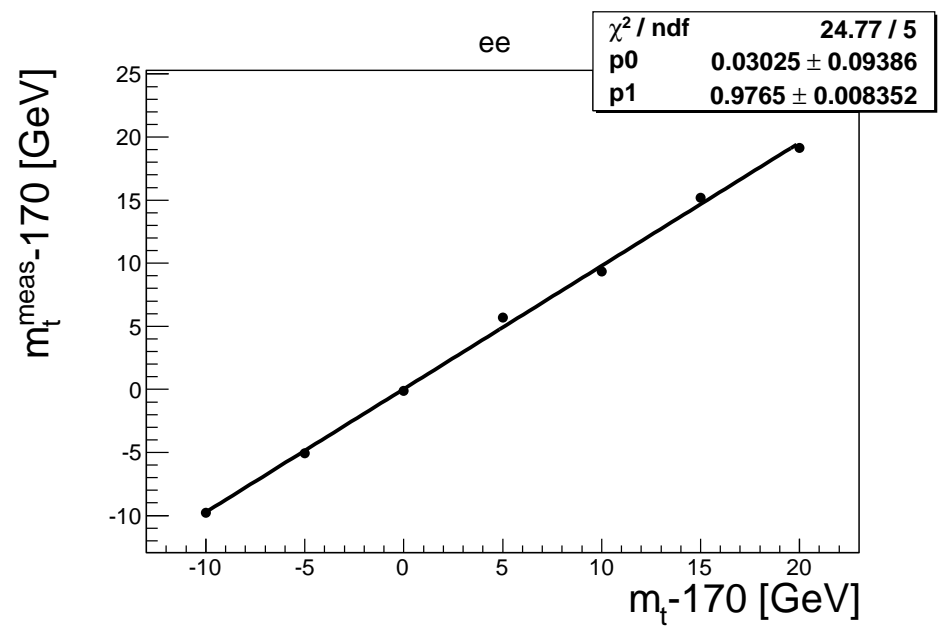

Figure 7.10. An example of the calibration points and the linear fit applied for ee (top) channel. The effects contributing to a high $\chi^{2}$ are not accounted for. 

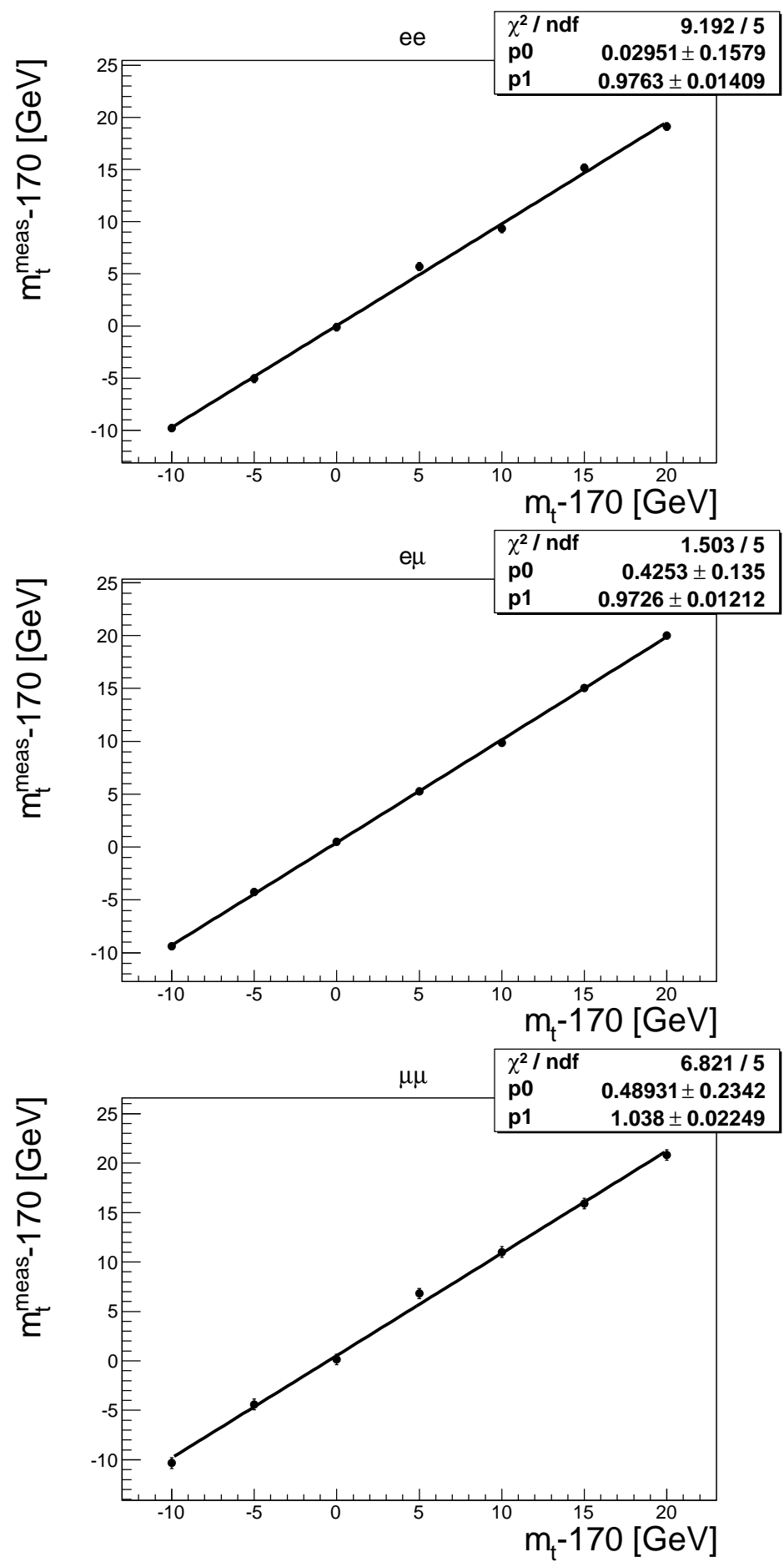

Figure 7.11. The calibration points and the linear fit applied for ee (top), e $\mu$ (center) and $\mu \mu$ (bottom) channel. An additional uncertainty due to finite template statistics is added in quadrature with statistical uncertainty at every mass point. 


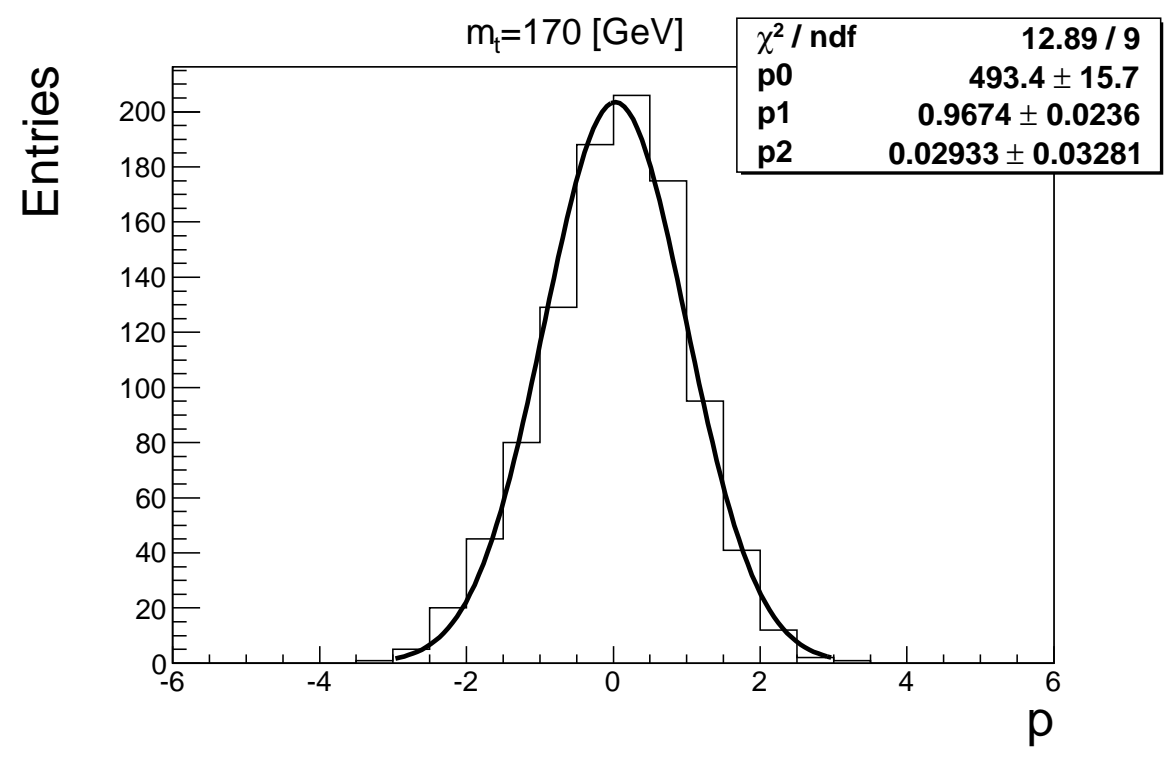

Figure 7.12. Pull distribution for the input top quark mass $m_{t}=170 \mathrm{GeV}$ in the $e \mu$ channel. The superimposed curve is the fit using a Gauss function. 


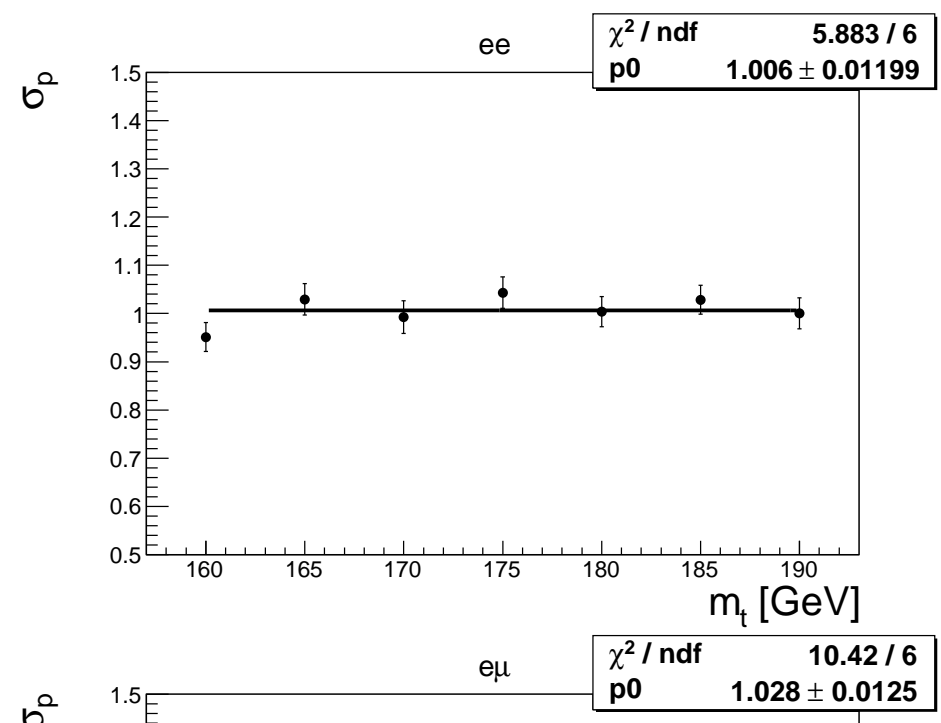

b

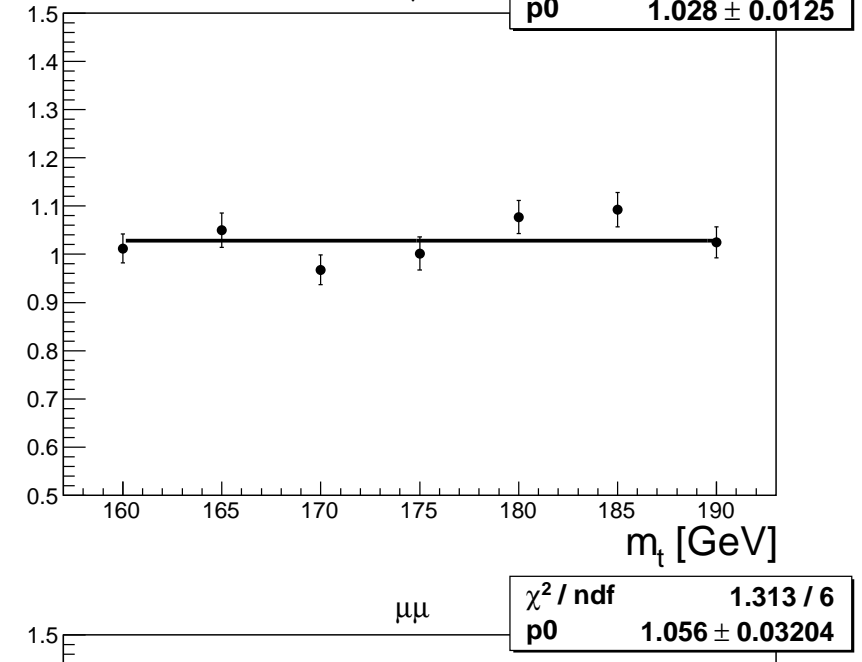

$0^{2}$

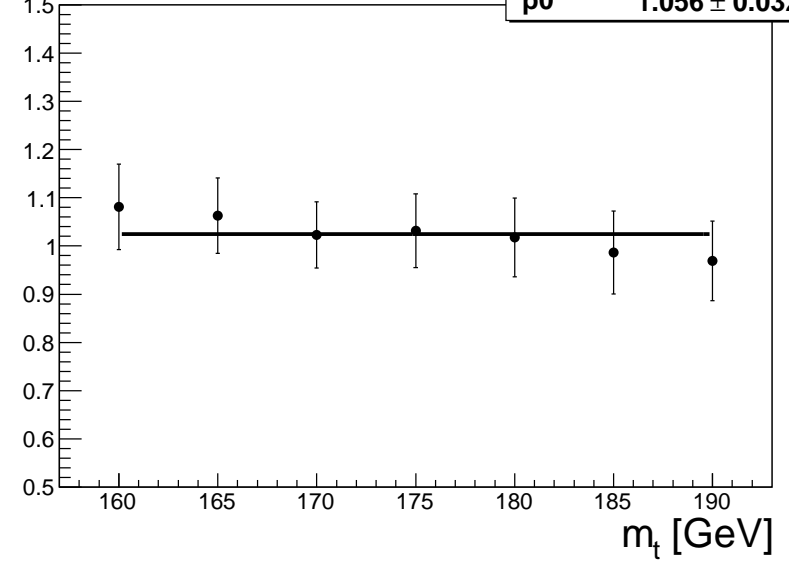

Figure 7.13. The pull calibration points and the linear fit applied for ee (top), e $\mu$ (center) and $\mu \mu$ (bottom) channel. 


\section{Chapter 8}

\section{MEASUREMENT OF THE TOP QUARK MASS}

\subsection{Event Selections}

The analysis of this thesis uses events with dilepton final state of the $t \bar{t}$ decay. In particular, the final state at the parton level is given by the decay: $t \bar{t} \rightarrow \ell \bar{\ell}^{\prime} \nu \bar{\nu}^{\prime} b \bar{b}$. The resulting final state of the $t \bar{t}$ event at the detector level therefore contains two isolated high- $p_{T}$ charged leptons, two energetic jets and the large $\vec{E}_{T}$.

Both the detector and the parton level final state of the $t \bar{t}$ event are not unique and can be consistent with the final states from the different background processes. The main background processes resulting in the similar final state are those that contain one or two gauge bosons $\left(W^{ \pm}\right.$or $Z$ ) and jets coming from ISR. The physics processes include diboson $(W W, Z Z$ and $W Z)$ and $Z$ boson production.

In the $W W$ diboson events, leptonic decays of $W$ bosons produce in the final state two isolated leptons and two neutrinos exactly as in the case of $t \bar{t}$. The jets in the final state, however, come from a parton initial state radiation. Although the ISR jets are not as energetic on average as $b$-jets, the cross section is large enough that significant $p_{T}$ jet production results. In the $W Z$ and $Z Z$ diboson events, two isolated high- $p_{T}$ leptons can be also produced in association with jets and thus mimic the detector final state from the $t \bar{t}$ decay.

Another background for the $t \bar{t}$ decay is $Z / \gamma^{*} \rightarrow \tau \tau$ production. Being short-lived particles, each of the $\tau$ subsequently decays into lepton and neutrino, $\tau \rightarrow \ell \nu$ where $\ell=e, \mu$. Coming from the same $Z / \gamma^{*}$, the two leptons tend to have lower $p_{T}$ than 
leptons from $t \bar{t}$ decay. Analogous to the diboson case, the two low- $p_{T}$ jets are coming from ISR. As a result, the detector final state of $Z / \gamma^{*}$ production is consistent with the final state of the $t \bar{t}$ decay. It is the largest background $e \mu$ channel of $t \bar{t}$. The background from direct $Z$ decay to two pair of electrons or muons, $Z \rightarrow e e$ and $Z \rightarrow \mu \mu$, is dominated in $e e$ and $\mu \mu$ channels.

An additional source of the contributing background is instrumental. Instrumental background originates from particle misidentification. For example, a misidentified electron can be mimicked by a jet in $W+$ jets or multijet events. An isolated muon can arise from the semileptonic decay of a heavy flavor hadron in a jet if the jet has not been reconstructed.

Event selection criteria are designed to reduce the ratio of the number of top quark events to the number of background events. The goal of the event selection criteria for the analysis of this thesis is to select events arising from $t \bar{t}$ decay while rejecting background events with the similar parton level final state. The selections are almost identical to that used for the measurement of the $t \bar{t}$ cross section in dilepton channels. The core data quality and trigger requirements for event objects - electron, muon and jet - can be obtained from [113].

The main cuts in the selections are made on the global event quantities like $p_{T}$, $H_{T}, \not_{T}$ and $\sigma_{\mathscr{H}_{T}}$. The $\mathscr{E}_{T}$ and the $\mathbb{E}_{T}$ significance $\sigma_{\not_{T}}$ are described in Section 5.2.6 and Section 8.3.4.4 respectively. The $H_{T}$ variable is the scalar sum of the transverse momenta of the leading lepton and the two leading jets. The minimal value a variable can have for an event to pass the selection is called its cut value.

A cut on the $H_{T}$ variable is very effective in rejecting $Z / \gamma^{*} \rightarrow \tau \tau$ and diboson backgrounds that are the largest backgrounds for $t \bar{t}$ in the $e \mu$ channel. The $\mathbb{E}_{T}$ variable cut effectively rejects $Z / \gamma^{*}$ backgrounds in the $e e$ and $\mu \mu$ channels. Finally, a cut on $\sigma_{\not_{T}}$ is very powerful to suppress events with non-zero $\mathbb{E}_{T}$ that originates not from underlying 
physical processes but rather from resolution fluctuations.

The requirements that are common for all channels are that for leptons $p_{T}^{\ell}>15$ $\mathrm{GeV}$, and jets $p_{T}^{\text {jet }}>20 \mathrm{GeV}$. Additionally to the transverse momenta requirements, the selections based on the object pseudorapidities are made. Electrons are accepted in the interval of pseudorapidities $|\eta|<2.5$, excluding the ICD region of the detector $1.1<|\eta|<1.5$. Muons are required to satisfy $|\eta|<2.0$ while jets must be within $|\eta|<2.5$.

The rest of the $t \bar{t}$ dilepton event selections slightly differ for each channel. In the ee channel, the cut on the $\mathscr{E}_{T}$ significance is $\sigma_{\mathscr{H}_{T}}>5.0$. The $e \mu$ channel is required to satisfy $H_{T}>120 \mathrm{GeV}$. The selection for the $\mu \mu$ channel is given by two selections: $E_{T}>40 \mathrm{GeV}$ and $\sigma_{\mathscr{H}_{T}}>$ 5.0. For every channel, final topological selections are chosen in such way that the expected statistical uncertainty on the measured top quark mass is minimal. All kinematic selections on global event quantities are summarized in Table 8.1 .

Table 8.1. The global variables event selections for $t \bar{t}$ dilepton channels. An entire event is rejected if at least one of the variables has a value below the specified cut value.

\begin{tabular}{c|cccccc}
\hline \hline Channel & $p_{T}^{e}$ & $p_{T}^{\mu}$ & $p_{T}^{j e t}$ & $H_{T}$ & $\mathbb{E}_{T}$ & $\sigma_{\mathscr{H}_{T}}$ \\
\hline \hline$e e$ & 15 & - & 20 & - & - & 5 \\
\hline$e \mu$ & 15 & 15 & 20 & 120 & - & - \\
\hline$\mu \mu$ & - & 15 & 20 & - & 40 & 5 \\
\hline \hline
\end{tabular}


An additional requirement not used in the $t \bar{t}$ cross-section measurement in the dilepton channel is that an event must also pass the kinematic reconstruction described in Section 7.1. Events that have empty weight distributions are removed from consideration since the distribution moment values are left undetermined. This introduces kinematic reconstruction inefficiency that does not exist in the cross-section measurement. The kinematic reconstruction efficiencies for the $e \mu$ channel before $H_{T}$ cut applied are shown in Table 8.2. The event yields for the dilepton channels after global selections and kinematic reconstruction are given in Table 8.3.

Table 8.2. The signal and background kinematic reconstruction efficiencies for he $e \mu$ channel with no $H_{T}$ cut applied. The efficiency for signal has been estimated on a $t \bar{t}$ sample of input top quark mass, $m_{t}=170 \mathrm{GeV}$.

\begin{tabular}{c|cccc}
\hline \hline Channel & $\varepsilon^{t \bar{t}}$ & $\varepsilon^{Z \rightarrow \ell \ell}$ & $\varepsilon^{\text {diboson }}$ & $\varepsilon^{\text {data }}$ \\
\hline \hline$e \mu$ & $98.7 \%$ & $91.0 \%$ & $94.7 \%$ & $97.9 \%$ \\
\hline \hline
\end{tabular}

\footnotetext{
${ }^{1}$ The ee channel instrumental background yield is taken from the dilepton cross section analysis [114].
} 
Table 8.3. Expected and observed event yields and their statistical uncertainty for $t \bar{t}$ dilepton channels after kinematic reconstruction and global selections applied. The expected yields have been derived on simulated MC signal and background sample. The instrumental background and its yield are derived from the data. The observed event yield is obtained using the events from data.

\begin{tabular}{c|cccccc}
\hline \hline Channel & $t \bar{t}$ & $Z \rightarrow \ell \ell$ & diboson & instrumental & total & data \\
\hline \hline$e e$ & $32.95 \pm 0.3$ & $8.1 \pm 1.1$ & $2.2 \pm 0.3$ & $0.1^{1}$ & $43.3 \pm 1.1$ & 49 \\
\hline$e \mu$ & $138.7 \pm 0.6$ & $10.6 \pm 1.1$ & $5.6 \pm 0.7$ & $8.95 \pm 3.6$ & $163.9 \pm 3.9$ & 190 \\
\hline$\mu \mu$ & $44.6 \pm 0.5$ & $24.5 \pm 2.7$ & $3.2 \pm 0.6$ & $4.5 \pm 1.0$ & $76.8 \pm 3.0$ & 80 \\
\hline \hline
\end{tabular}

\subsection{Data Measurement}

The top quark mass measurement presented in this thesis is performed using the $\nu \mathrm{WT}$ method on $4.3 \mathrm{fb}^{-1}$ of data collected by the $\mathrm{D} \varnothing$ experiment. The mass and statistical uncertainty are extracted with the maximum likelihood technique described in Section 7.3. The possibility for an event to have more than two jets indicates that one or more jets are originated from ISR/FSR. In this case, most energetic jets are selected as the $b$ jets for the neutrino weight calculation. Each dilepton channel is considered separately. The final likelihood over all channels is referred to as combined and is obtained by multiplying the likelihoods of these channels

$$
L^{c}\left(m_{t}\right)=L^{e e}\left(m_{t}\right) \cdot L^{e \mu}\left(m_{t}\right) \cdot L^{\mu \mu}\left(m_{t}\right)
$$

Thus the combined negative log-likelihood is 


$$
-\ln \left(L^{c}\left(m_{t}\right)\right)=\sum_{c h}\left(-\ln \left(L^{c h}\left(m_{t}\right)\right)\right)
$$

where "ch" denotes the set of channels, i.e. $e e, e \mu$ and $\mu \mu$. The resulting simultaneous measurement of the mass for the all three dilepton channels are obtained by minimizing the combined negative $\log$-likelihood $-\ln \left(L^{c}\left(m_{t}\right)\right)$. The final likelihood and the negative log-likelihoods for each individual dilepton channel are shown in Figure 8.1.

The uncertainty of the data measurement is corrected for deviations of the pull width from unity as well as for the slope and offset of the calibration curve. The calibrated and pull-corrected measurement of the top quark mass and its statistical uncertainty for the dilepton channels are given as

$$
\begin{gathered}
e e: m_{t}=170.3 \pm 6.4 \text { (stat.) } \mathrm{GeV} \\
e \mu: m_{t}=174.2 \pm 3.2 \text { (stat.) } \mathrm{GeV} \\
\mu \mu: m_{t}=183.8 \pm 18.0 \text { (stat.) } \mathrm{GeV}
\end{gathered}
$$

The combination of the measurements in the dilepton channels with statistical uncertainty only is

$$
\text { combined : } m_{t}=173.7 \pm 2.8 \text { (stat.) } \mathrm{GeV}
$$

The distributions of calibrated pull-corrected statistical uncertainties for the input top quark mass $m_{t}=170 \mathrm{GeV}$ from the pseudoexperiment testing are shown in Figure 8.2. For the $e e, e \mu$ channels and all three channel combination, the statistical uncertainties are in a good agreement with the predictions. The statistical uncertainty in $\mu \mu$ channel is consistent with the expectation at the probability level of about $7 \%$.

\subsection{Systematic Uncertainties}



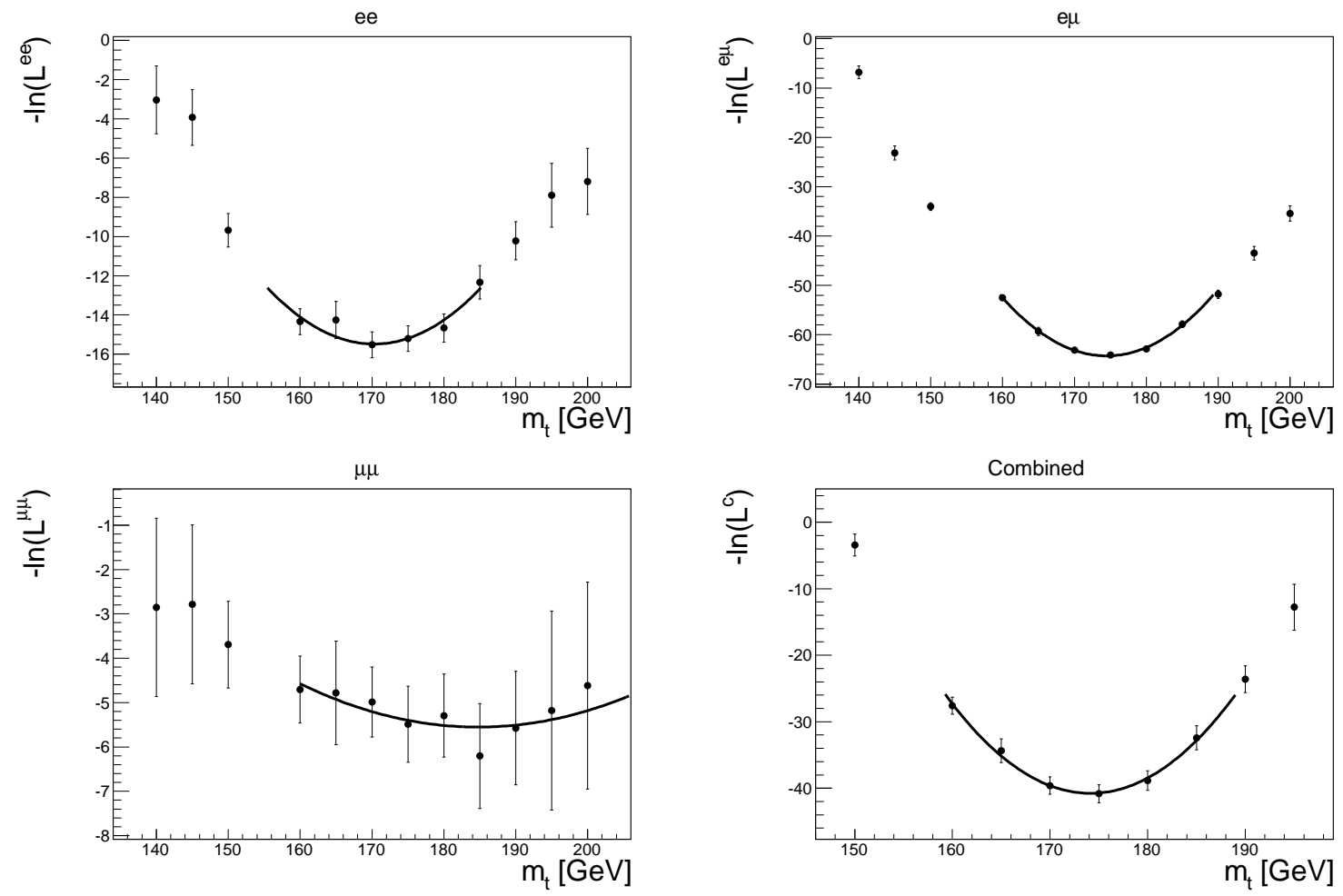

Figure 8.1. The $-\ln \left(L\left(m_{t}\right)\right)$ as a function of input top quark mass for the ee (top left), $e \mu$ (top right), $\mu \mu$ (bottom left) channels and their combination (bottom right). The black curve is a parabolic fit near the minimum value in $m_{t}$. 

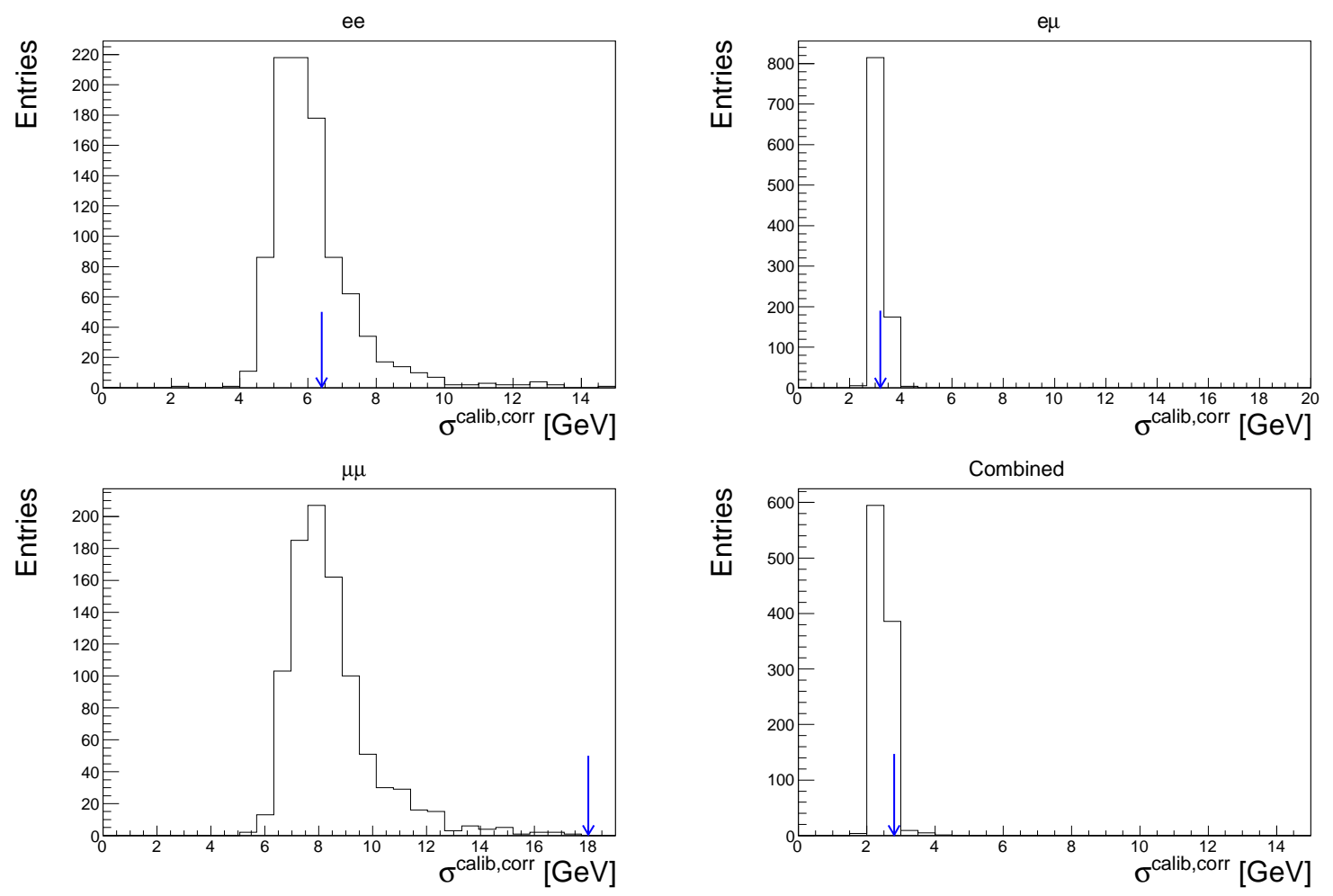

Figure 8.2. Expected calibrated and pull-corrected statistical uncertainty distributions for the ee (top left), e $\mu$ (top right), $\mu \mu$ (bottom left) channels and their combination (bottom right. The distributions are obtained from pseudoexperiment testing for the input top quark mass $m_{t}=170 \mathrm{GeV}$. Arrows indicate measured top quark mass in data. 
The statistical uncertainty calculated with the maximum likelihood method corresponds to random, or stochastic, fluctuations of a measured quantity. The stochastic nature reflects that the measurement is based on a finite number of observations. In terms of the top quark mass measurement in the dilepton channel, the finite number of observations corresponds to a finite number of $t \bar{t}$ events observed. If the measurement is performed on the same number of $t \bar{t}$ events but from a different dataset, it would result in a different measured mass of the top quark.

As opposed to the statistical uncertainty, the systematic uncertainties do not arise from the effect of a limited statistics but rather are the result of inaccurate equipment used, assumptions made in a method, imperfect calibration and others. Common systematic effects leading to the systematic uncertainty for many high energy physics analyses include effects such as background, energy and momentum resolution, selection bias, detector modeling, trigger efficiency etc. The systematic uncertainties are reproducible inaccuracies and do not change with repeated measurements of the same quantity.

Often the systematic uncertainties are of comparable scale to the statistical uncertainties. This results that the overall uncertainty in a measurement is dominated by the systematic uncertainties. Thus the identification and treatment of systematic uncertainties is an important aspect of almost any analysis in the high energy physics.

\subsubsection{Treatment of systematic uncertainties}

For the top quark mass measurement with te $\nu \mathrm{WT}$ method in $t \bar{t}$ dilepton final states, the systematic uncertainties are divided into four categories. The first category deals with uncertainties associated with the jet energy calibration. It includes the uncertainties due to effects of the overall energy scale, remaining residual biases from the $p_{T}$ and $\eta$ dependence, and the differenced in response of the detector to jets in data vs. MC 
simulation. The second category comprises effects from the QCD modeling of $t \bar{t}$ production. This involves the uncertainties in simulating additional jets due to ISR/FSR, QCD color reconnection in the context of the string fragmentation model, uncertainties from modeling of $b$-quark fragmentation, effects from next-to-leading order contributions, and choice of the parton distribution functions. The third category concerns the object reconstruction and identification. The uncertainties in this category are due to effects from the jet reconstruction and identification efficiency as well as the energy resolution of jets and leptons. The last category includes the systematics related to the $\nu \mathrm{WT}$ method itself. It is comprised of uncertainties from the method calibration (see Equation (7.32)), limited MC statistics used to build the templates, and the expected fraction of the signal events in the dataset.

Conventionally, there are two types of systematic uncertainties depending on the method used to estimate them. An uncertainty of the first type is due to the effect from changing relevant parameter value $q$ by $\pm \sigma_{q}$, where $\sigma_{q}$ is the standard deviation of the parameter. An uncertainty of the second type arises from the difference between models. Let us denote as $m_{t}^{0}$ the top quark mass measurement corresponding to the central value of the parameter $q$ for the uncertainty of the first type, or the default model for the uncertainty of the second type. The measurement obtained from variation of $q$ by $\pm \sigma_{q}$ is denoted as $m_{t}^{+}$and $m_{t}^{-}$. In case of the second type uncertainty, $m_{t}^{+}$and $m_{t}^{-}$correspond to the measured mass using the different model. If the alternative model gives a measurement which is greater than $m_{t}^{0}$, it is denoted as $m_{t}^{+}$, otherwise as $m_{t}^{-}$. The uncertainty of the first type is also referred as Type $I$ and the second type as Type II.

Systematic uncertainties are computed differently depending on the relationship between $m_{t}^{-}, m_{t}^{0}$ and $m_{t}^{+}$, as described in Ref. [115]. The systematic uncertainty $\delta m_{t}$ of either Type I or Type II is computed as follows 
- if $m_{t}^{-}<m_{t}^{0}<m_{t}^{+}$or $m_{t}^{+}<m_{t}^{0}<m_{t}^{-}$, then $\delta m_{t}=\left|m_{t}^{+}-m_{t}^{-}\right| / 2$

- if $\operatorname{sign}\left(m_{t}^{+}-m_{t}^{0}\right)=\operatorname{sign}\left(m_{t}^{-}-m_{t}^{0}\right)$, then $\delta m_{t}=\max \left(\left|m_{t}^{+}-m_{t}^{0}\right| / 2,\left|m_{t}^{-}-m_{t}^{0}\right| / 2\right)$

- if $m_{t}^{+}>0$ and $m_{t}^{-}$does not exist, $\delta m_{t}=\left|m_{t}^{+}-m_{t}^{0}\right|$

- if $m_{t}^{-}>0$ and $m_{t}^{+}$does not exist, $\delta m_{t}=\left|m_{t}^{-}-m_{t}^{0}\right|$

This approach in computing systematic uncertainties symmetrizes asymmetric uncertainties $^{2}$ in a conservative direction. All systematic uncertainties are required [115] to be symmetric to calculate the Tevatron top quark mass combination from the results of the CDF and D $\varnothing$ collaborations.

To calculate most of the systematic uncertainties for the analysis of this thesis, one or two additional MC simulated samples were required. The samples are generated with the same input top quark mass but for a different model that leads to a systematic effect. To produce MC samples for these studies, the value of the input top quark mass close to the world average is chosen, $m_{t}=172.5 \mathrm{GeV}$. However, there are systematic uncertainties that are obtained by modifying the value of a relevant parameter by $\pm \sigma_{q}$. In such cases, we don't need to generate new MC sample.

The list of the systematic uncertainties calculated in this analysis is given in Table 8.4. Due to the limited MC statistics of the systematics signal samples, the results in Table 8.4 for the color reconnection and higher order effects systematics are performed only for the $e \mu$ channel and corrected over to the $e e$ and $\mu \mu$ channels. All systematic and statistical uncertainty within the same channel are considered uncorrelated. The total systematic uncertainties for each channel and the combination are calculated as all

\footnotetext{
${ }^{2}$ The systematic uncertainties usually are not distributed according to a Gaussian function, i.e. asymmetric. Thus it is impossible to use the standard technique, i.e. adding deviations separately in quadrature, to obtain a combination of different systematics. One of the solutions that allows to use the standard approach is to make the uncertainties symmetric.
} 
individual uncertainties added in quadrature. To calculate total uncertainty statistical uncertainty are added in quadrature with the total systematic uncertainty. The values in Table 8.4 are rounded to $0.1 \mathrm{GeV}$ precision. While calculating the total systematic uncertainty the numbers were rounded to $10 \mathrm{MeV}$, however the final result is mostly given with $0.1 \mathrm{GeV}$ precision.

The following sections provide a description of each individual source of systematic uncertainty. The value of the combined systematic uncertainty for all dilepton channels is quoted at the end of every section.

\subsubsection{Jet Energy Scale}

\subsubsection{Overall scale}

Systematic uncertainty of this type results from the effects of carrying over the $\ell+$ jets jet energy scale to the dilepton events. The overall systematic effect on $\ell+$ jets scale $k_{J E S}$ is comprised of the following sources:

- In the $\ell+$ jets analysis [103], the jet energy scale factor is found to be $k_{J E S}=$ $1.013 \pm 0.008$ (stat). The statistical uncertainty equals $0.8 \%$ of the mean value and is the largest contribution to the systematic uncertainty, $\varepsilon_{k_{J E S}}^{\text {stat }}=0.8 \%$.

- The double ratio $\mathcal{R}_{2 \ell}^{b}$ for $b$-jets is slightly shifted from unity due to the different $b$-jet responses in $\ell+$ jets and dilepton samples. It varies in the range from 1.001 to 1.003. The maximum excursion of $\mathcal{R}_{2 \ell}^{b}$ from one is taken as $0.3 \%$ change in the $k_{J E S}$ mean value, $\varepsilon_{k_{J E S}}^{\text {topo }}=0.3 \%$.

- The jet energy scale in [103] is obtained using a different dataset than that used for my analysis. The dilepton sample is collected at the higher instantaneous luminosity that results in a different number of primary vertices. To estimate the contribution due to this effect, the distribution of the number of primary vertices 
Table 8.4. Summary of the systematic uncertainties of all dilepton channels and their combination dived in categories. The uncertainties are quoted in GeV.

\begin{tabular}{|c|c|c|c|c|}
\hline \multirow{2}{*}{ Source } & \multicolumn{4}{|c|}{ Channel } \\
\hline & $e e$ & $e \mu$ & $\mu \mu$ & combined \\
\hline \multicolumn{5}{|l|}{ Jet Energy Scale: } \\
\hline Overall scale & \pm 1.0 & \pm 0.85 & \pm 0.9 & \pm 0.9 \\
\hline Flavor Dependence & \pm 0.6 & \pm 0.5 & \pm 0.7 & \pm 0.5 \\
\hline Residual scale & \pm 0.35 & \pm 0.25 & \pm 0.45 & \pm 0.3 \\
\hline \multicolumn{5}{|l|}{ QCD Modeling: } \\
\hline ISR/FSR & \pm 0.1 & \pm 0.4 & \pm 0.9 & \pm 0.4 \\
\hline Color reconnection & \pm 0.5 & \pm 0.5 & \pm 0.5 & \pm 0.5 \\
\hline Higher order effects & \pm 0.6 & \pm 0.6 & \pm 0.6 & \pm 0.6 \\
\hline$b$-quark fragmentation & \pm 0.4 & \pm 0.1 & \pm 0.5 & \pm 0.1 \\
\hline PDF uncertainty & \pm 0.4 & \pm 0.5 & \pm 0.4 & \pm 0.5 \\
\hline \multicolumn{5}{|c|}{ Object Reconstruction and Identification: } \\
\hline Electron energy scale & \pm 0.6 & \pm 0.1 & - & \pm 0.2 \\
\hline Muon energy scale & - & \pm 0.2 & \pm 0.2 & \pm 0.2 \\
\hline Muon $p_{T}$ resolution & - & \pm 0.2 & \pm 0.2 & \pm 0.2 \\
\hline Jet resolution & \pm 0.2 & \pm 0.3 & \pm 0.1 & \pm 0.3 \\
\hline Jet identification & \pm 0.5 & \pm 0.3 & \pm 0.4 & \pm 0.3 \\
\hline \multicolumn{5}{|l|}{ Method: } \\
\hline Calibration & \pm 0.2 & \pm 0.1 & \pm 0.2 & \pm 0.1 \\
\hline Template Statistics & \pm 1.3 & \pm 0.5 & \pm 7.6 & \pm 0.5 \\
\hline Signal fraction & \pm 0.2 & \pm 0.1 & \pm 0.3 & \pm 0.2 \\
\hline Total uncertainty & \pm 2.2 & \pm 1.6 & \pm 7.9 & \pm 1.5 \\
\hline
\end{tabular}


in $\ell+$ jets data is re-weighted to match the distribution in dilepton sample. The effect of higher luminosity causes the change in mean value of $k_{J E S}$ by $0.01 \%$. This produces a negligible contribution to the systematic uncertainty.

- The calorimeter response changes with time. The $\ell+$ jets analysis is based on 2.6 $\mathrm{fb}^{-1}$ of data while analysis of this thesis uses $4.3 \mathrm{fb}^{-1}$. The time dependent shift in jet response was known for the newer data. To estimate a possible shift in the energy scale of the calorimeter, the events in $\ell+$ jets analysis has been re-weighted to match the jet energy response in the dilepton sample. The shift in $k_{J E S}$ is found to be $0.7 \%, \varepsilon_{k_{J E S}}^{L A r}=0.7 \%$.

All the sources leading to systematic shift in $k_{J E S}$ are considered as independent of one another, and therefore the overall systematic shift can bis obtained by

$$
\varepsilon_{k_{J E S}}^{\text {total }}=\sqrt{\varepsilon_{k_{J E S}}^{\text {stat }}{ }^{2}+\varepsilon_{k_{J E S}}^{\text {topo }}{ }^{2}+\varepsilon_{k_{J E S}}^{L A r^{2}}}=1.1 \%
$$

The new MC samples for signal and background are produced with $k_{J E S}$ shifted up and down by $1.1 \%$ of the mean value 1.013 . The mass measurement on the new MC sample is compared to the default one, giving an overall scale systematic uncertainty of $0.9 \mathrm{GeV}$.

\subsubsection{Residual scale}

The $\ell+$ jets scale factor $k_{J E S}$ is averaged over the $p_{T}$ 's and $\eta$ 's of jets from $W \rightarrow j j$ decay. This factor addresses a global scale difference between data and MC simulation. However, the possible effect from scale dependence on $p_{T}$ and $\eta$ may result in a nonnegligible systematic uncertainty that is called residual scale. To estimate the residual scale uncertainty the approach analogous to Ref. [116] is employed.

The $p_{T}$ and $\eta$ dependence of $k_{J E S}$ is derived from the standard jet energy scale obtained for the $\gamma+$ jets events. The standard jet energy scale correction is denoted as 
$J E S$ and the uncertainty on it is $\sigma_{J E S}$. For several $\eta^{j e t}$ bins, the fractional uncertainty $\sigma_{J E S} / J E S$ is parameterized as a function of the jet $p_{T}$. According to the JES group, the parameterization is as follows

$$
\frac{\sigma_{J E S}}{J E S}\left(p_{T}^{j e t} \mid \eta^{j e t}\right)=p_{1}+p_{2} \cdot p_{T}^{j e t}+p_{3} \cdot \exp \left(-p_{T}^{j e t} / p_{4}\right)
$$

where $p_{1}, p_{2}$, and $p_{3}$ are the fit parameters.

The assumed dependence of $k_{J E S}$ on $p_{T}^{\text {jet }}$ for every $\eta^{\text {jet }}$ bin is given by

$$
k_{J E S}\left(p_{T}^{j e t} \mid \eta^{j e t}\right)= \pm\left(\frac{\sigma_{J E S}}{J E S}\left(p_{T}^{j e t} \mid \eta^{j e t}\right)-\left\langle\frac{\sigma_{J E S}}{J E S}\left(p_{T}^{j e t} \mid \eta^{j e t}\right)\right\rangle\right)\left\langle k_{J E S}\right\rangle+\left\langle k_{J E S}\right\rangle
$$

where $\left\langle\frac{\sigma_{J E S}}{J E S}\left(p_{T}^{j e t} \mid \eta^{j e t}\right)\right\rangle$ is fractional uncertainty averaged over $p_{T}^{\text {jet }}$ range for given $\eta^{\text {jet }}$ bin, and $\left\langle k_{J E S}\right\rangle$ is the $\ell+$ jets global scale factor that is equal 1.013. The \pm sign in front of the parenthesis reflects two possible ways in defining $p_{T}^{j e t}$ dependence of $k_{J E S}$. The plus (minus) sign corresponds to the correction shifted up (down). The average fractional uncertainty is subtracted from $\frac{\sigma_{J E S}}{J E S}\left(p_{T}^{\text {jet }} \mid \eta^{j e t}\right)$ to extract the actual shape of the dependence, and thus the averaged over $p_{T}^{\text {jet }}$ range $\left\langle k_{J E S}\left(p_{T}^{j e t} \mid \eta^{j e t}\right)\right\rangle$ equals $\left\langle k_{J E S}\right\rangle$ as expected.

To estimate the residual scale systematic uncertainty, the analysis is re-run with varied scale factor. The energy of jets in the samples are shifted up and down according to Equation (8.9). The measured mass of the top quark on the the new samples is compared to the default one, and the uncertainty is found to be $0.3 \mathrm{GeV}$.

\subsubsection{Flavor Dependence}

To finish bringing the job of the simulation of jet response into agreement with that observed in data, the flavor-dependent correction is applied to all MC samples of this analysis. In the previous iteration of the top quark mass measurement with 
$\nu \mathrm{WT}$ method [109], the difference in the responses between $b$ and light jets led to an additional systematic uncertainty, called b/light response ratio. In this analysis, the flavor-dependent correction properly accounts for the different responses of $b$ quark jets, light quark jets, and gluon jets between data and MC. It therefore eliminates the need for $b /$ light response uncertainty. However, this correction has its own uncertainties that must be propagated. The flavor-dependent systematic uncertainty arises from the uncertainties in single-particle responses in data and MC. Details on the flavor-dependent correction used in the analysis of this thesis can be found in Section 6.2.1.

To estimate the flavor-dependent systematic uncertainty, the correction factors are shifted by one standard deviation up and down. The modified flavor corrections are applied to the light jets and $b$ jets in events from $t \bar{t} \mathrm{MC}$ sample. The uncertainty is computed to be $0.5 \mathrm{GeV}$.

\subsubsection{QCD Modeling}

\subsubsection{ISR/FSR}

The systematic uncertainty of this type arises from variations in modeling of the parton showering process in the initial and final-state radiation. The evaluation of the ISR/FSR systematic is performed by comparing three MC $t \bar{t}$ samples. The samples are produced with the PYTHIA generator for the same input top quark mass but with different values of the ISR/FSR parameters. The values are taken from the ISR/FSR study at CDF [118]. The CDF collaboration had determined the parameters variation range by comparing the PYTHIA MC to data. Thus for the anaysis of this thesis, one of the samples is produced with default PYTHIA parton shower parameters and the two other samples with parameters shifted by plus (minus) one standard deviation up (down). The set of PDF functions used is from CTEQ5L [117]. The resulting systematic 
due to ISR/FSR modeling is found to be $0.3 \mathrm{GeV}$.

\subsubsection{Color reconnection}

The strong interactions between the underlying event and the hard-scattering is known as the color reconnection process. The MC samples used in this thesis are generated without this effect taken into the account. However, the study carried out in Ref. [119] shows that the top quark mass is noticeably sensitive to the choice of color reconnection.

To estimate the possible effect due to the color reconnection modeling, two $\mathrm{MC} t \bar{t}$ samples are produced with and without explicit color reconnections. The samples are generated using PYTHIA v6.4 tune Apro and PYTHIA v6.4 tune ACRpro [120]. The ACRpro tune includes color reconnection modeling while the Apro tune does not. The resulting difference on the measured top quark mass between the two tunes is $0.5 \mathrm{GeV}$.

\subsubsection{Higher order effects}

The MC $t \bar{t}$ samples used for the analysis of this thesis are produced with the ALPGEN event generator which is interfaced with PYTHIA for showering and hadronization. ALPGEN calculates the matrix element of the hard-scattering process to leading order Feynman diagrams. This does not include such higher-order effects as contribution from $g g$ initial state, additional radiation of hard jets etc. To address the uncertainty related to the higher order effects, ALPGEN is compared with the next-to-leading order MC generator MC@NLO [121].

The two additional MC signal samples with the same input top quark mass were produced using ALPGEN and MC@NLO. Unlike for the default $t \bar{t}$ samples used for the measurement calibration, showering and hadronization processes are simulated with HERWIG. The choice of using HERWIG is dictated by consistency requirement since 
MC@NLO can only be interfaced with HERWIG. Comparing ALPGEN+PYTHIA vs. MC@NLO+HERWIG certainly would double-count fragmentation effects studied in Section 8.3.3.4, because PYTHIA and HERWIG model the parton showering and hadronization differently. To isolate and measure the effects from higher order corrections, showering and hadronization need to be simulated with the same generator. The difference between LO and NLO generators leads to the systematic effect that is estimated to be $0.6 \mathrm{GeV}$.

\subsubsection{4. b-quark fragmentation}

This systematic uncertainty arises from possible effects in modeling of the $b$-quark fragmentation. To simulate different choices of $b$-quark fragmentation models, the $t \bar{t}$ events are reweighted, and the default PYTHIA $b$-fragmentation function is replaced with Bowler fragmentation function [122]. The default MC samples are re-skimmed with the Bowler fragmentation function input parameters tuned to data collected at the LEP collider [123], and to data from SLAC collider. The resulting difference in the measurement carried on these two reweighted samples yields a systematic uncertainty of $0.1 \mathrm{GeV}$.

\subsubsection{PDF uncertainty}

The choice of the parton distribution functions includes a systematic uncertainty. The default signal MC samples are produced using the CTEQ6L1 set of PDFs. Possible effects from the different PDF choice are studied by reweighting the default $t \bar{t}$ events. The systematic uncertainty is evaluated by performing one standard deviation up and down for each of the 20 parton distribution functions from CTEQ6M [124]. The appropriate systematic uncertainty is estimated according to that recommended in Ref. [124]: 


$$
\delta m_{t}=\frac{1}{2}\left(\sum_{i=1}^{N_{p}}\left(m_{t}\left(S_{i}^{+}\right)-m_{t}\left(S_{i}^{-}\right)\right)^{2}\right)^{1 / 2}
$$

where $i$ runs over the set of PDFs, $N_{p}$ is the total number of PDFs $\left(N_{p}=20\right), m_{t}\left(S_{i}^{+}\right)$and $m_{t}\left(S_{i}^{-}\right)$are the measured top quark masses for the positive and the negative excursion by one standard deviation respectively of the $i$-th PDF. The result is found to be 0.5 $\mathrm{GeV}$.

\subsubsection{Object Reconstruction and Identifications}

\subsubsection{Electron energy scale}

The electron energy scale in default MC samples does not accurately match the respective value in data. As found by the $\mathrm{D} \varnothing W$ mass group, the difference between the energy scale for an electron in MC and data arises from the mismodeling of the material in the detector. The data is better described by adding extra material to the solenoid that provides electron radiation losses corresponding to $0.25 X_{0}$. To account for an extra material and thus achieve a better description of data by MC simulation, an additional correction to the electron energy scale needs to be applied. The difference between the standard electron scale and the new one that takes into the account an additional material in solenoid yields a systematic uncertainty of $0.2 \mathrm{GeV}$

\subsubsection{Muon energy scale}

Similarly to the case of the electron energy scale, the muon momentum scale measured in data does not exactly match value from MC. The values of the average dilepton invariant mass measured in $Z \rightarrow \mu \mu$ and $J / \psi \rightarrow \mu \mu$ decays are not identical in MC and data [125]. This indicates a relative difference in the muon momentum scale between $\mathrm{MC}$ and data. 
Two different scaling functions, linear and quadratic, have been implemented to correct muon $p_{T}$, and default $t \bar{t}$ MC samples were re-skimmed. The systematic effect is evaluated by comparing the results from corrected and default samples. The difference between the standard muon energy scale and the one that gives largest deviation is taken to be the systematic uncertainty. The uncertainty is found to be $0.2 \mathrm{GeV}$.

\subsubsection{Muon transverse momentum resolution}

The muon $p_{T}$ resolution in default MC samples does not exactly match the resolution from data. To bring MC muon resolution in agreement with that in data, additional smearing to Monte Carlo events is applied [125]. Smearing correction posses some uncertainty that allows for potential systematic effect on the top quark mass measurement.

To evaluate the systematic uncertainty due to this effect, the smearing parameters have been shifted by one standard deviation up and down. The mass measurement on the re-skimmed MC samples with the shifted and default scaling function parameters gives the systematic uncertainty estimate. The uncertainty is estimated to be $0.2 \mathrm{GeV}$.

\subsubsection{Jet energy resolution}

Although MC jets are reconstructed using the same algorithms as for data, they do not exactly reproduce the jets in data due to the number of reasons. These include the detector mismodeling, approximations made in the models of parton showering and hadronization processes, and others. As the result, MC simulated jets have slightly higher energies and better energy resolution. To address the issue of improper jet simulation, an additional correction is applied to the MC samples. The samples are corrected by shifting and smearing the jet energies as described in [126]. The correction is also known as JSSR (jet smearing, shifting and removing). 
To evaluate the systematic uncertainty due to the effect of the jet resolution, the parameters of the jet energy smearing function obtained from $\gamma+$ jets events in data are modified by one standard deviation up and down of their uncertainties. The altered scenarios were compared to the default configuration. The uncertainty is found to be $0.3 \mathrm{GeV}$

\subsubsection{Jet identification}

Due to the various assumptions made at the stage of the jet MC simulation, jet multiplicity in data does not exactly agree with the MC simulation. The jet reconstruction algorithm has slightly different reconstruction efficiencies for data and MC. As it has been found, the MC simulated jets tend to have more efficient reconstruction besides an

overall better energy resolution. As a result, higher jet reconstruction efficiencies lead to a larger number of jets identified in $\mathrm{MC}$ simulation that those in data.

To achieve a better agreement between data and MC simulation, the JSSR correction is applied. The scale factors used in the JSSR to smear jet energies, however, are estimated within some uncertainty. The systematic effect due to these scaling uncertainties is estimated varying the corresponding scale factors by their uncertainties. The default MC samples with parameters modified up and down by one standard deviation are re-skimmed. The mass measurement on these samples are compared to the default scenario, and the systematic uncertainty is found to be $0.3 \mathrm{GeV}$.

\subsubsection{Systematics of the Method}

\subsubsection{Calibration}

An uncertainty of the $\nu \mathrm{WT}$ method arises from the uncertainties on the calibration parameters in Equation (7.32). The parameters, the slope and offset, are determined by 
fitting the calibration points obtained from the pseudoexperiment testing to a straight line. An example of the calibration plots fitted with the linear fit are shown in Figure 7.11 for all three dilepton channels.

Due to the finite statistics of Monte Carlo samples, every calibration point has some statistical uncertainty associated with it. The statistical fluctuations of the calibration points result in the uncertainties of the fit parameters correspondingly. The systematic uncertainty due to the calibration can be estimated using the standard formula for propagation of errors. Thus, for example, if the uncertainties on the slope and offset are defined as $\delta \alpha$ and $\delta \beta$ respectively, then according to the formula of propagation of errors, the uncertainty on the top quark mass can be written as follows

$$
\delta m_{t}^{\text {calib,meas }}=\sqrt{\left(\frac{\partial m_{t}^{\text {calib,meas }}}{\partial \alpha}\right)^{2} \delta \alpha^{2}+\left(\frac{\partial m_{t}^{\text {calib,meas }}}{\partial \beta}\right)^{2} \delta \beta^{2}}
$$

After plugging in the explicit expression of the derivatives, the uncertainty is given by

$$
\delta m_{t}^{\text {calib,meas }}=\frac{1}{\alpha^{2}} \sqrt{\left(m_{t}^{\text {meas }}-\beta-170\right)^{2} \delta \alpha^{2}+\alpha^{2} \delta \beta^{2}}
$$

Taking the values of $\delta \alpha$ and $\delta \beta$ from results of the fit, the calibration systematic uncertainty is estimated to be $0.1 \mathrm{GeV}$. Table 8.5 provides calibration systematic uncertainty for each channel with and without point-to-point fluctuations from template statistics accounted for.

\subsubsection{Template Statistics}

Systematic uncertainty of this type arises from the limited MC statistics employed to construct probability density histograms, or templates. Each individual bin of the templates acquires a statistical uncertainty within which the bin content is unknown. 
Table 8.5. Calibration systematic uncertainty with template statics accounted for (right), and without it (left).

\begin{tabular}{l|cc}
\hline \hline \multirow{2}{*}{ Channel } & \multicolumn{2}{|c}{ Calibration uncertainty $[\mathrm{GeV}]$} \\
\cline { 2 - 3 } & without template statistics & with template statistics \\
\hline$e e$ & 0.09612 & 0.16169 \\
$e \mu$ & 0.04761 & 0.13889 \\
$\mu \mu$ & 0.18242 & 0.22606 \\
combined & 0.03874 & 0.13059 \\
\hline \hline
\end{tabular}

Statistical fluctuations of a bin content may cause a significant shift in the value of the top quark mass measured and therefore needs to be thoroughly estimated.

To evaluate the effect of the template bin fluctuations, a thousand measurements are performed using the $\mathrm{D} \varnothing$ data but with templates fluctuated to sample the errors in the template. The bin content in these templates is varied by sampling a Gaussian function with sigma equal to the bin statistical uncertainty, and the bin mean value is taken as a default bin value. The root-mean-square of the results from a thousand data measurements on the new templates yields a template statistics systematic of $0.5 \mathrm{GeV}$.

\subsubsection{Signal fraction}

Due to the finite MC statistics used in the $\nu \mathrm{WT}$ method calibration, the signal and background yields are determined within some statistical uncertainty. While the relative uncertainty on the expected number of signal events is small, the uncertainty for the backgrounds is substantial. Thus the actual fraction of signal events depends on the background yield uncertainty. The average signal and background fractions affect the 
measured top quark mass when constructing the likelihood function in Equation (7.23) and also at the time of ensemble testing when forming pseudoexperiments.

To estimate the possible systematic effect of the statistical uncertainty on background event yield, the mean yields of all background and signal processes are varied by their uncertainties up and down. The pseudoexperiment studies are performed using the shifted event yields to derive new calibration curves. Subsequently, the new calibrations are used to correct the top quark mass measurement in data. The systematic effect is evaluated by comparing the data measurements obtained with the new and default calibrations. The uncertainty is calculated to be $0.2 \mathrm{GeV}$. 


\section{Chapter 9}

\section{SUMMARY AND OUTLOOK}

This thesis presents the measurement of the top quark pole mass in dilepton final states with the neutrino weighting method. The measurement is performed using the $\nu \mathrm{WT}$ event reconstruction on $4.3 \mathrm{fb}^{-1}$ of data followed by the maximum likelihood fit. The analyzed data was collected by the $\mathrm{D} \varnothing$ detector from $p \bar{p}$ collisions at $\sqrt{s}=1.96$ $\mathrm{TeV}$ from the Fermilab Tevatron collider. The fit is applied to the first two moments

of the resulting distribution of relative weight as a function of the top quark mass. The measurement gives,

$$
\begin{aligned}
& e e: m_{t}=170.3 \pm 6.4 \text { (stat.) } \pm 1.85 \text { (syst.) } \mathrm{GeV} \\
& e \mu: m_{t}=174.2 \pm 3.2 \text { (stat.) } \pm 1.6 \text { (syst.) } \mathrm{GeV} \\
& \mu \mu: m_{t}=183.8 \pm 18.0 \text { (stat.) } \pm 6.4 \text { (syst.) } \mathrm{GeV}
\end{aligned}
$$

The combination of the measurements in the dilepton channels is

$$
\text { combined: } m_{t}=173.7 \pm 2.8 \text { (stat.) } \mathrm{GeV} \pm 1.5 \text { (syst.) } \mathrm{GeV}
$$

The measurement is combined with the result from the preceding measurement [109] on $1 \mathrm{fb}^{-1}$ of the $\mathrm{D} \varnothing$ data. To produce the combination, best linear unbiased estimator (BLUE) $\operatorname{method}^{1}[127],[128]$ is used yielding

\footnotetext{
${ }^{1}$ The BLUE method is useful to produce combination of several measurements with correlated uncertainties. Each measurement is assigned a weight that is calculated using the measurements' covariance matrix. The unbiased estimator of measured quantity is given as weighted sum of all measurements. The uncertainties are expressed in terms weights and the correlation coefficients.
} 


$$
m_{t}=174.0 \pm 2.4 \text { (stat.) } \mathrm{GeV} \pm 1.4 \text { (syst.) } \mathrm{GeV}
$$

As seen from Figure 9.1, the result is consistent with measurements in other channels. To date, this is the most precise measurement of the top quark mass in dilepton channel at publication [129]. Figure 9.2 shows the mass of an updated results of the $W$ boson versus mass of the top quark from Ref. [130]. The orange contour is the constraint on $m_{W}$ and $m_{t}$ at $68 \%$ confidence level based on the data from Tevatron and LEP-II as of summer 2012. The result is also consistent with the SM model prediction of the Higgs mass in the range $115.5<m_{H}<127 \mathrm{GeV}$.

The statistical uncertainty of the measurement can be further reduced by analyzing the full $\mathrm{D} \varnothing$ dataset of about $10 \mathrm{fb}^{-1}$. By using two times larger dataset, the statistical uncertainty is expected to drop by a factor of $\sqrt{2}$. Additionally, the reduction of the statistical uncertainty can be also possible due to improvements in the analysis method itself. For example, such improvements as ISR/FSR jet separation, or the template bin fluctuations decrease. The jet separation can be performed by assigning relative weight to the jets in events with more than two jets, as it is done in [106]. The decrease in the template bin fluctuations can be achieved by employing larger number of MC simulated events. 


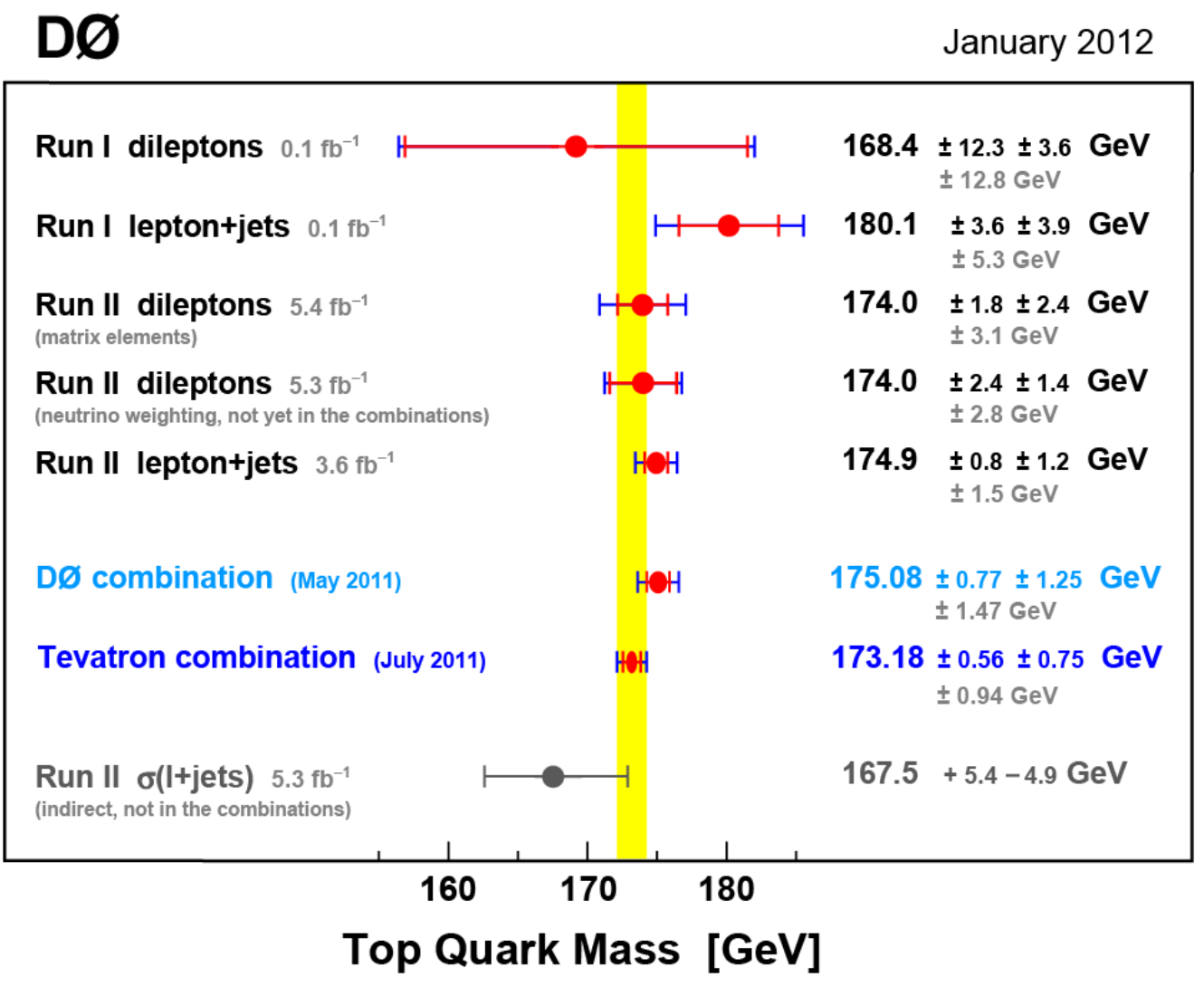

Figure 9.1. Summary of the top quark mass measurement in different decay channels at DØ. The D $\varnothing$ combination and Tevatron average of the top quark mass [131]. The mass extracted from the cross section measurement is shown for comparison and has not been used in the combination. 


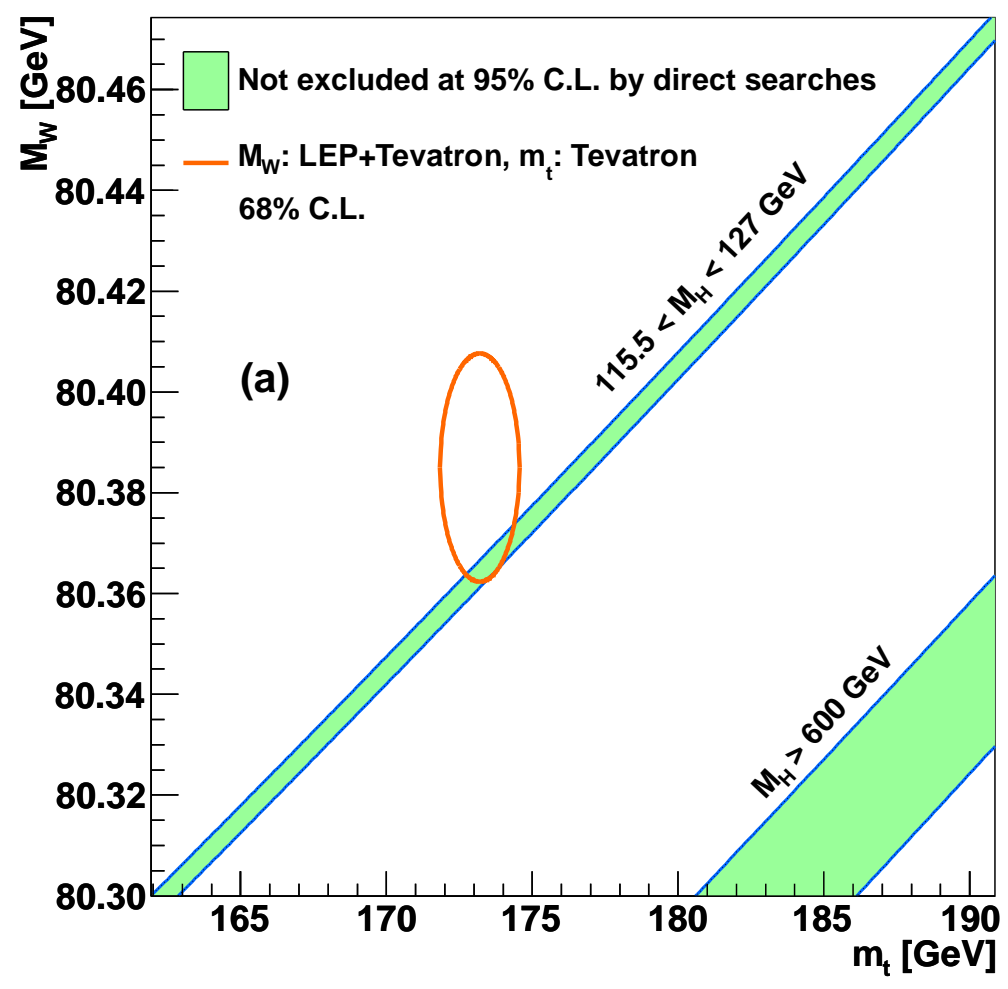

Figure 9.2. The indirect constraints on $m_{t}$ and $m_{W}$ (orange ellipse) from LEP-II and Tevatron data as of July 2012. Green bands are the SM relationship for the masses as a function of the Higgs mass not excluded by the direct searches $\left(115.5<m_{H}<127 \mathrm{GeV}\right.$ and $\left.600<m_{H}<1000 \mathrm{GeV}\right)$. 


\section{Appendix A \\ MUON QUALITY REQUIREMENTS}

Appendix A outlines muon quality requirements. The B and C layers are considered together and called the $B C$ layer.

- Tight muons.

$-|n s e g|=3$

- at least two A layer wire hits

- at least one A layer scintillator hit

- at least three BC layer wire hits

- at least one BC scintillator hit

- a converged local fit $\left(\chi^{2}>0\right)$

- Medium muons.

$-|n s e g|=3$

- at least two A layer wire hits

- at least one A layer scintillator hit

- at least two BC layer wire hits

- at least one $\mathrm{BC}$ scintillator hit (except for the muons in the central region $|\eta|<1.4$ that have less than four $\mathrm{BC}$ wire hits)

or 
$-n s e g=2$

- at least one BC layer scintillator hit

- at least two BC layer wire hits

- located in the octant 5 and 6 with $\left|\eta^{\text {detector }}\right|<1.6$

or

$-n s e g=1$

- at least one scintillator hit

- at least two A layer wire hits

- located in the octant 5 and 6 with $\left|\eta^{\text {detector }}\right|<1.6$

- Loose muons. An $|n s e g|=3$ Loose muon is defined as a Medium muon with one of tests from the list below failed. The A wire and scintillator requirement treated as one test and requiring always at least one scintillator.

$-|n s e g|=3$

- at least two A layer wire hits

- at least one A layer scintillator hit

- at least two BC layer wire hits

- at least one BC scintillator hit (except for the muons in the central region $|\eta|<1.4$ that have less than four BC wire hits)

or

$-n s e g=2$

- at least one BC layer scintillator hit

- at least two BC layer wire hits 
$-n s e g=1$

- at least one scintillator hit

- at least two A layer wire hits. 


\section{Appendix B \\ MUON ISOLATION WORKING POINTS}

- TopScaledUltraLoose $=$ ScaledTrackHalo $<1.0$ and ScaledCalorimeter Halo $<$ 1.0

- TopScaledVeryLoose $=$ ScaledTrackHalo $<0.5$ and ScaledCalorimeter Halo $<$ 0.5

- TopScaledLoose $=$ ScaledTrackHalo $<0.2$ and ScaledCalorimeter Halo $<0.2$

- TopScaledMedium $=$ ScaledTrackHalo $<0.15$ and ScaledCalorimeterHalo $<$ 0.15

- TopScaledTight $=$ ScaledTrackHalo $<0.1$ and ScaledCalorimeter Halo $<0.1$

- TopScaledVeryTight $=$ ScaledTrackHalo $<0.05$ and ScaledCalorimeter Halo $<$ 0.05

- TopP14 = ScaledTrackHalo $<0.06$, ScaledCalorimeter Halo $<0.08$, and $\Delta R(\mu$, jet $)>$ 0.5

- $\operatorname{DeltaR}=\Delta R(\mu, j e t)>0.5$

- NPLoose $=$ TrackHalo $<4.0 \mathrm{GeV}$ and Calorimeter Halo $<2.5 \mathrm{GeV}$

- NPTight $=$ TrackHalo $<2.5 \mathrm{GeV}$ and Calorimeter Halo $<2.5 \mathrm{GeV}$ 


\section{Appendix C}

\section{THE PULL VARIABLE PROBABILITY DENSITY FUNCTION}

\section{C.1. Ideal Scenario}

For a selected pseudoexperiment under a given mass hypothesis $m_{t}$, the pull variable is defined through the calibrated top quark mass estimate $m_{t}^{\text {meas, calib }}$ and its calibrated statistical uncertainty, $\sigma^{\text {meas, calib }}$. According to its definition in Equation (7.38), the pull variable is constructed as follows

$$
p \equiv \frac{m_{t}^{\text {meas }, \text { calib }}-m_{t}}{\sigma^{\text {meas }, \text { calib }}}
$$

Both $m_{t}^{\text {meas,calib }}$ and $\sigma^{\text {meas, }}$ calib are derived in the pseudoexperiment using the likelihood technique and consequently calibrated. The distribution of pulls can be obtain by performing many pseudoexperiments. For the analysis presented in this thesis, a thousand pseudoexperiments are performed for a given top quark mass hypothesis.

In the ideal case, the measured top quark mass $m_{t}^{\text {meas, calib }}$ has to be distributed around its true value $m_{t}$ by the Gaussian distribution

$$
f\left(m_{t}^{\text {meas }, \text { calib }}\right)=\frac{1}{\sqrt{2 \pi \sigma^{2}}} \exp \left(-\frac{\left(m_{t}^{\text {meas }, \text { calib }}-m_{t}\right)^{2}}{2 \sigma^{\text {true }}{ }^{2}}\right)
$$

and the calibrated statistical error $\sigma^{\text {meas, calib }}$ to be equal width of the distribution $\sigma^{\text {true }}$, i.e.

$$
\sigma^{\text {meas, }} \text { calib }^{\text {true }}
$$


To find the probability density function for pulls $g(p)$, the change of variables technique is used. According to it, the $g(p)$ and $f\left(m_{t}^{\text {meas, }}\right.$ calib $)$ are related as follows

$$
g(p)=f\left(m_{t}^{\text {meas, }{ }^{2} \text { alib }}(p)\right)\left|\frac{\mathrm{d} m_{t}^{\text {meas, calib }}(p)}{\mathrm{d} p}\right|
$$

The dependence of $m_{t}^{\text {meas, }}$ calib as a function of $p$ can be obtained from Equation (C.1)

$$
m_{t}^{\text {meas, } \text { calib }}(p)=p \sigma^{\text {meas, } \text { calib }}+m_{t}
$$

and therefore the derivative is

$$
\frac{\mathrm{d} m_{t}^{\text {meas, calib }}(p)}{\mathrm{d} p}=\sigma^{\text {meas }, \text { calib }}
$$

Changing the variables in Equation (C.2) from $m_{t}^{\text {meas, calib }}$ to $p$, the expression for

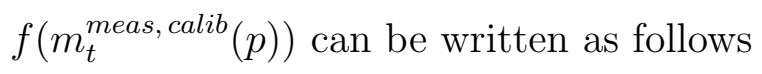

$$
f\left(m_{t}^{\text {meas }, \text { calib }}(p)\right)=\frac{1}{\sqrt{2 \pi \sigma^{\text {true }}}} \exp \left(-\frac{\left(p \sigma^{\text {meas }, \text { calib }}\right)^{2}}{2 \sigma^{\text {true }}{ }^{2}}\right)
$$

By plugging Equation (C.6) and (C.7) in (C.4), the probability density for the pull is found to be

$$
g(p)=\frac{\sigma^{\text {meas }, \text { calib }}}{\sqrt{2 \pi \sigma^{\text {true }}}} \exp \left(-\frac{\left(p \sigma^{\text {meas }, \text { calib }}\right)^{2}}{2 \sigma^{\text {true }}{ }^{2}}\right)
$$

Since in the ideal scenario $\sigma^{\text {meas,calib }}=\sigma^{\text {true }}$, the pull probability density can be simplified, leading to the final expression as follows

$$
g^{\text {ideal }}(p)=\frac{1}{\sqrt{2 \pi}} \exp \left(-\frac{p^{2}}{2}\right)
$$

Thus the pull probability density in the ideal case for a given top quark mass hypothesis is a Gauss function with the mean value equal zero and the width equal one. 


\section{C.2. Non-Ideal Scenario}

Unlike the ideal case, in practice, the pull distribution for a given top quark mass hypothesis is distributed by a Gaussian with a width different from one. This indicates that the calibrated statistical uncertainty $\sigma^{\text {meas,calib }}$ is not calibrated precisely enough and an additional correction needs to be applied.

In the non-ideal case, the calibrated statistical uncertainty is related to the unbiased one by

$$
\sigma^{\text {true }} \simeq \bar{k} \sigma^{\text {meas, } \text { calib }}
$$

where $\bar{k}$ is the averaged over many pseudoexperiments correction factor for a given top quark mass hypothesis.

Equation (C.10) can be re-written as

$$
\sigma^{\text {meas }, \text { calib }} \simeq \frac{\sigma^{\text {true }}}{\bar{k}}
$$

Thus to obtain the probability density function $g(p)$ for the non-ideal scenario, Equation (C.11) is combined with Equation (C.8) leading to the following expression

$$
g(p)=\frac{\sigma^{\text {true }}}{\bar{k} \sqrt{2 \pi \sigma^{\text {true }} 2}} \exp \left(-\frac{\left(p \sigma^{\text {true }}\right)^{2}}{2\left(\bar{k} \sigma^{\text {true }}\right)^{2}}\right)
$$

that can be further simplified and $g(p)$ in the non-ideal case for a given top quark mass hypothesis is given by

$$
g^{\text {non-ideal }}(p)=\frac{1}{\sqrt{2 \pi \bar{k}^{2}}} \exp \left(-\frac{p^{2}}{2 \bar{k}^{2}}\right)
$$

Thus the pull probability density in the non-ideal case for a given top quark mass hypothesis is a Gauss function with the non-unitary width $\bar{k}$ and the mean value equal zero. 
Appendix D

\section{PULL-CORRECTED CALIBRATED STATISTICAL UNCERTAINTY}

As it is shown in Appendix C, calibrated statistical uncertainty for a given top quark mass hypothesis in the non-ideal scenario is related to an unbiased one by Equation (C.10), i.e.

$$
\sigma^{\text {true }} \simeq \bar{k} \sigma^{\text {meas }, \text { calib }}
$$

In the measurement on a dataset, however, Equation (D.1) can not be applied directly. The value of the correction factor $\bar{k}$ depends on the top quark mass hypothesis that is not known for the case of collider data.

The study on Monte Carlo simulated data shows that pull distributions for different top quark mass hypotheses have the widths very close to each other. Thus when performing a measurement on the actual dataset, Equation (D.1) can be used if the correction factor $k$ is replaced by its average $\langle\bar{k}\rangle$

$$
\sigma^{\text {true }} \simeq\langle\bar{k}\rangle \sigma^{\text {meas, }} \text { calib }
$$

The average correction factor $\langle\bar{k}\rangle$ is defined as an averaged of $\bar{k}$ over many top quark mass hypotheses

$$
\langle\bar{k}\rangle=\frac{1}{N} \sum_{i=1}^{N} \bar{k}_{i}
$$

where $i$ runs over top quark mass hypotheses, and $N$ is the total number of the hypotheses. 
While doing the data measurement, the best approximation of $\sigma^{\text {true }}$ is defined as $\sigma^{\text {calib,corr }}$ and therefore Equation (D.2) is replaced by

$$
\sigma^{\text {calib,corr }} \simeq\langle\bar{k}\rangle \sigma^{\text {meas, calib }}
$$

In practice, one of the possible ways to derive $\langle\bar{k}\rangle$ that accounts for statistical deviations from $\bar{k}$ over range of the top quark mass hypotheses is to obtain $\langle\bar{k}\rangle$ from a linear fit. The width of a pull distribution is plotted versus top quark mass hypothesis for many pseudoexperiments. By fitting the plot to the constant straight line $p\left(m_{t}^{M C}\right)=\lambda$, the average $\langle\bar{k}\rangle$ is defined as follows

$$
\langle\bar{k}\rangle=\lambda
$$

Thus assuming exact equality, Equation (D.4) can be re-written by

$$
\sigma^{\text {calib,corr }}=\lambda \sigma^{\text {meas, calib }}
$$

Equation (D.6) is employed in the analysis of this thesis to correct the statistical uncertainty after it has been calibrated with the mass calibration curve. The derived uncertainty $\sigma^{\text {calib,corr }}$ is also known as pull-corrected calibrated statistical uncertainty. 


\section{REFERENCES}

[1] R. Barate et al. [LEP Working Group for Higgs boson searches and ALEPH and DELPHI and L3 and OPAL Collaborations], "Search for the standard model Higgs boson at LEP," Phys. Lett. B 565, 61 (2003).

[2] S. Chatrchyan et al. [CMS Collaboration], "Observation of a new boson at a mass of $125 \mathrm{GeV}$ with the CMS experiment at the LHC," Phys. Lett. B 716, 30 (2012).

[3] G. Aad et al. [ATLAS Collaboration], "Observation of a new particle in the search for the Standard Model Higgs boson with the ATLAS detector at the LHC," Phys. Lett. B 716, 1 (2012).

[4] S. Weinberg, "A Model of Leptons," Phys. Rev. Lett. 19, 1264 (1967).

[5] A. Salam, "Elementary Particle Theory," edited by N. Svarthholm (Almqvist, forlag AB, Stockholm, 1968), p. 367.

[6] W. Heisenberg, "The 'observable Quantities' In The Theory Of Elementary Particles," UCRL-TRANS-808 (1943).

[7] [ALEPH and CDF and D0 and DELPHI and L3 and OPAL and SLD and LEP Electroweak Working Group and Tevatron Electroweak Working Group and SLD Electroweak and Heavy Flavour Groups Collaborations], "Precision Electroweak Measurements and Constraints on the Standard Model" (2010).

[8] [ALEPH and DELPHI and L3 and OPAL and SLD and LEP Electroweak Working Group and SLD Electroweak Group and SLD Heavy Flavour Group Collaborations], "Precision electroweak measurements on the $Z$ resonance," Phys. Rept. 427, 257 (2006).

[9] P. L. Anthony et al. [SLAC E158 Collaboration], "Precision measurement of the weak mixing angle in Moller scattering," Phys. Rev. Lett. 95, 081601 (2005).

[10] P. J. Mohr, B. N. Taylor and D. B. Newell, "CODATA Recommended Values of the Fundamental Physical Constants: 2006," Rev. Mod. Phys. 80, 633 (2008). 
[11] H. D. Politzer, "Reliable Perturbative Results for Strong Interactions?," Phys. Rev. Lett. 30, 1346 (1973).

[12] S. Eidelman et al. [Particle Data Group Collaboration], "Review of particle physics. Particle Data Group," Phys. Lett. B 592, 1 (2004).

[13] O. Adriani et al. [L3 Collaboration], "Measurement of $\Gamma_{b \bar{b}} / \Gamma_{\text {had }}$ from hadronic decays of the Z," Phys. Lett. B 307, 237 (1993).

[14] O. Adriani et al. [L3 Collaboration], "Results from the L3 experiment at LEP," Phys. Rept. 236, 1 (1993).

[15] J. Bernabeu, A. Pich and A. Santamaria, "Top quark mass from radiative corrections to the $Z \rightarrow b \bar{b}$ decay," Nucl. Phys. B 363, 326 (1991).

[16] S. Abachi et al. [D0 Collaboration], "Observation of the top quark," Phys. Rev. Lett. 74, 2632 (1995).

[17] F. Abe et al. [CDF Collaboration], "Observation of top quark production in $\bar{p} p$ collisions," Phys. Rev. Lett. 74, 2626 (1995).

[18] F. Abe et al. [CDF Collaboration], "A Lower limit on the top quark mass from events with two leptons in $p \bar{p}$ collisions at $\sqrt{s}=1.8 \mathrm{TeV}$," Phys. Rev. Lett. 68, 447 (1992).

[19] S. Abachi et al. [D0 Collaboration], "Search for the top quark in $p \bar{p}$ collisions at $\sqrt{s}=1.8$ TeV," Phys. Rev. Lett. 72, 2138 (1994).

[20] T. E. W. Group [CDF and D0 Collaboration], "Combination of CDF and D0 Results on the Mass of the Top Quark," arXiv:0903.2503 [hep-ex].

[21] V. M. Abazov et al. [D0 Collaboration], "An Improved determination of the width of the top quark," Phys. Rev. D 85, 091104 (2012).

[22] V. M. Abazov et al. [D0 Collaboration], "Experimental discrimination between charge 2e/3 top quark and charge 4e/3 exotic quark production scenarios," Phys. Rev. Lett. 98, 041801 (2007).

[23] D. Chang, W. -F. Chang and E. Ma, "Alternative interpretation of the Tevatron top events," Phys. Rev. D 59, 091503 (1999).

[24] N. Kidonakis and R. Vogt, "The Theoretical top quark cross section at the Tevatron and the LHC," Phys. Rev. D 78, 074005 (2008).

[25] M. Cacciari, S. Frixione, M. L. Mangano, P. Nason and G. Ridolfi, "Updated predictions for the total production cross sections of top and of heavier quark pairs at the Tevatron and at the LHC," JHEP 0809, 127 (2008). 
[26] V. M. Abazov et al. [D0 Collaboration], "Measurements of single top quark production cross sections and $\left|V_{t b}\right|$ in $p \bar{p}$ collisions at $\sqrt{s}=1.96 \mathrm{TeV}$," Phys. Rev. D 84, $112001(2011)$

[27] M. Beneke and V. M. Braun, "Heavy quark effective theory beyond perturbation theory: Renormalons, the pole mass and the residual mass term," Nucl. Phys. B 426, 301 (1994).

[28] I. I. Y. Bigi, M. A. Shifman, N. G. Uraltsev and A. I. Vainshtein, "The Pole mass of the heavy quark. Perturbation theory and beyond," Phys. Rev. D 50, 2234 (1994).

[29] W. J. Marciano, "Precision electroweak measurements and the Higgs mass," eConf C 040802, L009 (2004).

[30] J. Alcaraz [ALEPH and CDF and D0 and DELPHI and L3 and OPAL and SLD Collaboration], arXiv:0911.2604 [hep-ex].

[31] T. E. W. Group [CDF and D0 Collaboration], "Updated Combination of CDF and D0 Results for the Mass of the W Boson," arXiv:0908.1374 [hep-ex].

[32] W. A. Bardeen, C. T. Hill and M. Lindner, "Minimal Dynamical Symmetry Breaking of the Standard Model," Phys. Rev. D 41, 1647 (1990).

[33] C. T. Hill, "Topcolor: Top quark condensation in a gauge extension of the standard model," Phys. Lett. B 266, 419 (1991).

[34] C. T. Hill, "Topcolor assisted technicolor," Phys. Lett. B 345, 483 (1995).

[35] V. M. Abazov et al. [D0 Collaboration], "Measurement of the $t \bar{t}$ production cross section using dilepton events in $p \bar{p}$ collisions," Phys. Lett. B 704, 403 (2011).

[36] V. M. Abazov et al. [D0 Collaboration], "Measurement of the top quark pair production cross section in the lepton+jets channel in proton-antiproton collisions at $\sqrt{s}=1.96$ TeV," Phys. Rev. D 84, 012008 (2011).

[37] V. Ahrens, A. Ferroglia, M. Neubert, B. D. Pecjak and L. L. Yang, "Renormalization-Group Improved Predictions for Top-Quark Pair Production at Hadron Colliders," JHEP 1009, 097 (2010).

[38] V. Ahrens, A. Ferroglia, M. Neubert, B. D. Pecjak and L. L. Yang, "Top-Quark Pair Production Beyond Next-to-Leading Order," Nucl. Phys. Proc. Suppl. 205206, 48 (2010).

[39] S. Moch and P. Uwer, "Theoretical status and prospects for top-quark pair production at hadron colliders," Phys. Rev. D 78, 034003 (2008). 
[40] U. Langenfeld, S. Moch and P. Uwer, "Measuring the running top-quark mass," Phys. Rev. D 80, 054009 (2009).

[41] N. Kidonakis and R. Vogt, "Next-to-next-to-leading order soft gluon corrections in top quark hadroproduction," Phys. Rev. D 68, 114014 (2003).

[42] N. Kidonakis, "Next-to-next-to-leading soft-gluon corrections for the top quark cross section and transverse momentum distribution," Phys. Rev. D 82, 114030 (2010).

[43] RunII Handbook. Fermilab Beams Division, March 2001. http://www-bd.fnal. gov/runII/index.html

[44] C. Schmidt and C. Curtis, "A 50-mA Negative Hydrogen-Ion Source", IEEE Transactions on Nuclear Science, NS-26, 4120 (1979).

[45] C. Curtis et al., "Linac H-Beam Operation and Uses at Fermilab", IEEE Transactions on Nuclear Science, NS-26, 3760 (1979).

[46] S. Nagaitsev, "Project X - a new multi-megawatt proton source at Fermilab", FERMILAB-CONF-11-092-AD (2011).

[47] E. Hubbard et al., "Booster synchrotron", Fermilab Technical Report Fermilab TM-405, (1973).

[48] C. S. Mishra, "The Fermilab main injector", In the Proceedings of Particles \& Fields 92: 7th Meeting of the Division of Particles Fields of the APS (DPF 92), Batavia, Illinois, 10-14 Nov 1992, pp 1619-1621 (1992).

[49] A. Ruggiero, "The Fermilab Tevatron I Debuncher Ring", IEEE Transactions on Nuclear Science, NS-30, 2478 (1983).

[50] J. Griffin et al., "Time and Momentum Exchange for Production and Collection of Intense Antiproton Beams at Fermilab", IEEE Transactions on Nuclear Science, NS-30, 2630 (1983).

[51] A. Ando, T. Collins and D. Johnson, "Design of an 8-GeV Accumulator Ring For the Fermilab Tevatron I Project", IEEE Transactions on Nuclear Science, NS-30, 2031 (1983).

[52] D. Mohl, G. Petrucci, L. Thorndahl and S. Van Der Meer, "Physics And Technique Of Stochastic Cooling", Phys. Rept. 58, 73 (1980).

[53] G. Jackson, "Recycler ring conceptual design report", FERMILAB-TM-1936. 
[54] S. Nagaitsev et al. [Electron Cooling Collaboration], "Antiproton cooling in the Fermilab Recycler Ring", AIP Conf. Proc. 821, 39 (2006).

[55] K. Johnsen, "The CERN intersecting storage rings", Nucl. Instrum. Meth. 108, 205 (1973).

[56] Group, TeVI, "Design Report Tevatron 1 project", FERMILAB-DESIGN-1984-01

[57] S. Abachi et al. [D0 Collaboration], "The D0 Detector", Nucl. Instrum. Meth. A 338, 185 (1994).

[58] V. M. Abazov et al. [D0 Collaboration], "The Upgraded D0 Detector", Nucl. Instrum. Meth. A 565, 463 (2006).

[59] J. Brzezniak et al., "Conceptual Design Of A 2-Tesla Superconducting Solenoid For The Fermilab D0 Detector Upgrade", FERMILAB-TM-1886;

[60] B. S. Acharya et al. [D0 Collaboration], "Scintillation counters for the D0 muon upgrade," Nucl. Instrum. Meth. A 401, 45 (1997).

[61] Type 404A plastic scintillator, Bicron Corporation, 12345 Kinsman Rd, Newbury, $\mathrm{OH}$ 44065-9677.

[62] Type BCF91A waveshifter fiber, Bicron Corporation, 12345 Kinsman Rd, Newbury, OH 44065-9677.

[63] THORN EMI, Gencom Inc, 23 Madison Rd., Fairfield, NJ 07006.

[64] The D0 Common Sample Group. http://www-d0.fnal.gov/Run2Physics/cs/ index.html

[65] R. Brun and F. Rademakers, "ROOT: An object oriented data analysis framework," Nucl. Instrum. Meth. A 389, 81 (1997).

[66] R. Brun and F. Rademakers, "ROOT: An object oriented data analysis framework", Linux Journal 51, (1998)

[67] Trigger Modeling for RunIIb. https://plone4.fnal.gov/P1/D0Wiki/tdaq/tsg/ triggerrun $2 b /$

[68] The DØ TriggerMeisters' page. http://www-dOonline.fnal.gov/www/groups/ $\mathrm{tm} / \mathrm{tm} \_$main.html

[69] T. Sjöstrand, S. Mrenna, P.Z. Skands, J. High Energy Phys. 0605 (2006) 026.

[70] M.L. Mangano, M. Moretti, F. Piccinini, R. Pittau, A.D. Polosa, J. High Energy Phys. 0307 (2003) 001. 
[71] R. Brun, F. Carminati, CERN Program Library Long Writeup W5013, 1993 (unpublished)

[72] J. Pumplin et al., J. High Energy Phys 207, 012 (2002) .

[73] T. Stelzer, W. F. Long, Automatic generation of tree level helicity amplitudes, Comput. Phys. Commun. 81, 357-371 (1994).

[74] G. Corcella et al., "HERWIG 6.5: an event generator for Hadron Emission Reactions With Interfering Gluons (including supersymmetric processes)," JHEP 0101, 010 (2001).

[75] B. Andersson, G. Gustafson, G. Ingelman and T. Sjostrand, "Parton Fragmentation And String Dynamics," Phys. Rept. 97, 31 (1983).

[76] B. R. Webber, "A QCD Model For Jet Fragmentation Including Soft Gluon Interference," Nucl. Phys. B 238, 492 (1984).

[77] S. Höche et al., "Matching Parton Showers and Matrix Elements," hepph/0602031.

[78] A. Khanov, "HTF: histogramming method for finding tracks. The algorithm description", D0 Note 3778.

[79] The D0 Algorithms Group. http://www-d0.fnal.gov/computing/algorithms/ index.html

[80] G. Borissov, "Ordering a Chaos or ... Technical Details of AA Tracking", presented at All DØ meeting, Feb. 28, 2003. http://wwwd0.fnal.gov/atwork/adm/ d0_private/2003-02-28/adm_talk.ps

[81] R. Duda, P. Hart, "Use of the Hough transformation to detect lines and curves in pictures", Communications of the ACM 15, 111972.

[82] R. Fruhwirth, "Application Of Kalman Filtering To Track And Vertex Fitting," Nucl. Instrum. Meth. A 262, 444 (1987).

[83] A. Schwartzman, C. Tully, "Primary vertex reconstruction by means of adaptive vertex fitting", D0 Note 4918.

[84] A. Schwartzman, M. Narain, "Probabilistic Primary Vertex Selection", D0 Note 4042 .

[85] A. Abdesselam, "Comparison of H-Matrices for electron identification in D0 Run II", D0 Note 3745. 
[86] M. Aoki, "Electron Likelihood in p20", D0 Note 5675.

[87] P. Calfayan et al., "Muon Identification Certification for p17 data", D0 Note 5157.

[88] G. Blazey et al., "Run II Jet Physics", D0 Note 3750.

[89] E. Busato, B. Andrieu, "Jet Algorithms in D0 RunII Software: Description and User's Guide", D0 Note 4457.

[90] G. Bernardi et al., "Improvements from the T42 Algorithm on Calorimeter Objects Reconstruction", D0 Note 4335.

[91] A. Harel et al., "Jet ID optimization", D0 Note 4919.

[92] A. Harel, R. Wagner "Improved L1 Confirmation", D0 Note 4932.

[93] S. Calvet et al., "Towards MissingET Certification and Unclustered Energy Studies", D0 Note 4927.

[94] A. Schwartzman, Fermilab-THESIS-2004-21. http://www-d0.fnal.gov/ results/publications_talks/thesis/schwartzman/thesis.pdf

[95] JES Group, "Jet Energy Scale Determination at D0 Run II (final p17 version)", D0 Note 5382 (2007).

[96] D. Gillberg et al., "Measuring Jet Response Using the Missing Et Projection Fraction Method in photon+jet Events", D0 Note 4571 (2004).

[97] R. Kehoe, "Resolution Bias in Jet Response Measurement", D0 Note 2052 (1994).

[98] R. Wigmans, "On The Energy Resolution Of Uranium And Other Hadron Calorimeters," Nucl. Instrum. Meth. A 259, 389 (1987).

[99] R. J. Barlow and C. Beeston, "Fitting using finite Monte Carlo samples," Comput. Phys. Commun. 77, 219 (1993).

[100] P. Renkel, Y. Ilchenko, R. Kehoe, "Measuring the top quark mass in the dilepton channel with $5.3 \mathrm{fb}^{-1}$, D0 Note 6187 (2011).

[101] Z. Ye et al., "Correction for the MC-Data Difference in the Jet Response at D0", D0 Note 6143 (2011).

[102] Z. Ye et al., "Correction for the MC-Data Difference in the Jet Response at D0 for Run IIB", D0 Note 6144 (2011).

[103] V. M. Abazov et al. [D0 Collaboration], "Precise measurement of the top-quark mass from lepton+jets events at D0," Phys. Rev. D 84, 032004 (2011) 
[104] E. Varnes, "Measurement of the Top Quark Mass", FERMILAB-THESIS-1997-28;

[105] B. Abbott et al. [D0 Collaboration], "Measurement of the top quark mass using dilepton events. DØ Collaboration," Phys. Rev. Lett. 80, 2063 (1998).

[106] B. Abbott et al. [D0 Collaboration], "Measurement of the top quark mass in the dilepton channel," Phys. Rev. D 60, 052001 (1999).

[107] V. M. Abazov et al. [D0 Collaboration], "Direct measurement of the mass difference between top and antitop quarks," Phys. Rev. D 84, 052005 (2011).

[108] A. N. Kolmogorov, "Grundbegriffe der Wahrscheinlichkeitsrechnung," published by Chelsea publishing company, New York, N.Y. (1946).

[109] V.M. Abazov et al. [D0 Collaboration], "Measurement of the top quark mass in final states with two leptons," Phys. Rev. D 80, 092006 (2009).

[110] R. J. Barlow, "Statistics: A Guide to the Use of Statistical Methods in the Physical Sciences," published by John Wiley \& Sons, Chichester, West Sussex, UK (1989).

[111] M. Mulders, "Ensemble testing for the Top Mass measurement", D0 Note 6144.

[112] R. J. Barlow, "Application of the Bootstrap resampling technique to Particle Physics experiments", perprint MAN-HEP-99-4 available at http://www.hep. man.ac.uk/preprints/1999.html

[113] F. Deliot, C. Deterre, A. Grohsjean, V. Sharyy, "Update of the ttbar Selection in Dilepton Final State for RunIIa, RunIIb1 and RunIIb2 Dataset", D0 Note 6127.

[114] M. Besancon et al., "Measurement of the $t \bar{t}$ Production Cross-Section in Dilepton Final State Using RunIIb1 and RunIIb2 Dataset for Winter 2010 Conferences", D0 Note 6027.

[115] F. Deliot et al., "Systematic Uncertainties in Top Quark Measurements", D0 Note 6024.

[116] Z. Ye et al., "Measurement of the Top Quark Mass in the Lepton+Jets Final State of Top Quark Pair Production Using Matrix Element Method on $2.6 \mathrm{fb}^{-1}$ D0 RunIIb Data", D0 Note 5853.

[117] H. L. Lai et al. [CTEQ Collaboration], "Global QCD analysis of parton structure of the nucleon: CTEQ5 parton distributions," Eur. Phys. J. C 12, 375 (2000).

[118] A. Abulencia et al. [CDF Collaboration], "Top quark mass measurement using the template method in the lepton + jets channel at CDF II," Phys. Rev. D 73, 032003 (2006). 
[119] P. Z. Skands and D. Wicke, "Non-perturbative QCD effects and the top mass at the Tevatron," Eur. Phys. J. C 52, 133 (2007).

[120] A. Buckley, H. Hoeth, H. Lacker, H. Schulz and J. E. von Seggern, "Systematic event generator tuning for the LHC," Eur. Phys. J. C 65, 331 (2010).

[121] S. Frixione and B. R. Webber, "Matching NLO QCD computations and parton shower simulations," JHEP 0206, 029 (2002).

[122] M. G. Bowler, "e+ e- Production of Heavy Quarks in the String Model," Z. Phys. C 11, 169 (1981).

[123] Y. Peters et al. [D0 Collaboration], "Precise tuning of the b fragmentation for the D0 Monte Carlo," FERMILAB-TM-2425-E.

[124] J. Pumplin, D. R. Stump, J. Huston, H. L. Lai, P. M. Nadolsky and W. K. Tung, "New generation of parton distributions with uncertainties from global QCD analysis," JHEP 0207, 012 (2002).

[125] M. Arthaud et al., "Muon Momentum Oversmearing for p20 Data", D0 Note 5449.

[126] N. Makovec and J.-F. Grivaz, "Shifting, Smearing and Removing Simulated Jets", D0 Note 4914.

[127] L. Lyons, D. Gibaut and P. Clifford, "How To Combine Correlated Estimates Of A Single Physical Quantity," Nucl. Instrum. Meth. A 270, 110 (1988).

[128] A. Valassi, "Combining correlated measurements of several different physical quantities," Nucl. Instrum. Meth. A 500, 391 (2003).

[129] V. M. Abazov et al. [D0 Collaboration], "Measurement of the top quark mass in $p \bar{p}$ collisions using events with two leptons," Phys. Rev. D 86, 051103 (2012).

[130] T. Aaltonen et al. [D0 Collaboration], "Combination of the top-quark mass measurements from the Tevatron collider," Phys. Rev. D 86, 092003 (2012).

[131] [Tevatron Electroweak Working Group and CDF and D0 Collaborations], "Combination of CDF and D0 results on the mass of the top quark using up to $5.8 \mathrm{fb}^{-1}$ of data," arXiv:1107.5255 [hep-ex]. 